\title{
Ultrafast transmission electron microscopy of a structural phase transition
}

\author{
DISSERTATION \\ zur Erlangung des mathematisch-naturwissenschaftlichen Doktorgrades \\ „Doctor rerum naturalium“ \\ der Georg-August-Universität Göttingen \\ im Promotionsprogramm ProPhys \\ der Georg-August University School of Science (GAUSS)
}

vorgelegt von

Thomas Christian Danz

aus Krefeld

Göttingen, 2021 
$\underline{\text { Betreuungsausschuss }}$

Prof. Dr. Claus Ropers, IV. Physikalisches Institut

und Max-Planck-Institut für biophysikalische Chemie

Prof. Dr. Christian Jooß, Institut für Materialphysik

Mitglieder der Prüfungskommission

Referent: $\quad$ Prof. Dr. Claus Ropers, IV. Physikalisches Institut und Max-Planck-Institut für biophysikalische Chemie

Korreferent: $\quad$ Prof. Dr. Christian Jooß, Institut für Materialphysik

2. Korreferent: Prof. Nuh Gedik, Ph.D., Department of Physics, Massachusetts Institute of Technology

Weitere Mitglieder der Prüfungskommission

Prof. Dr. Stefan Mathias, I. Physikalisches Institut

Prof. Dr. Vasily Moshnyaga, I. Physikalisches Institut

Prof. Dr. Michael Seibt, IV. Physikalisches Institut

Prof. Dr. Peter Sollich, Institut für Theoretische Physik

Tag der mündlichen Prüfung: 12. Juli 2021 


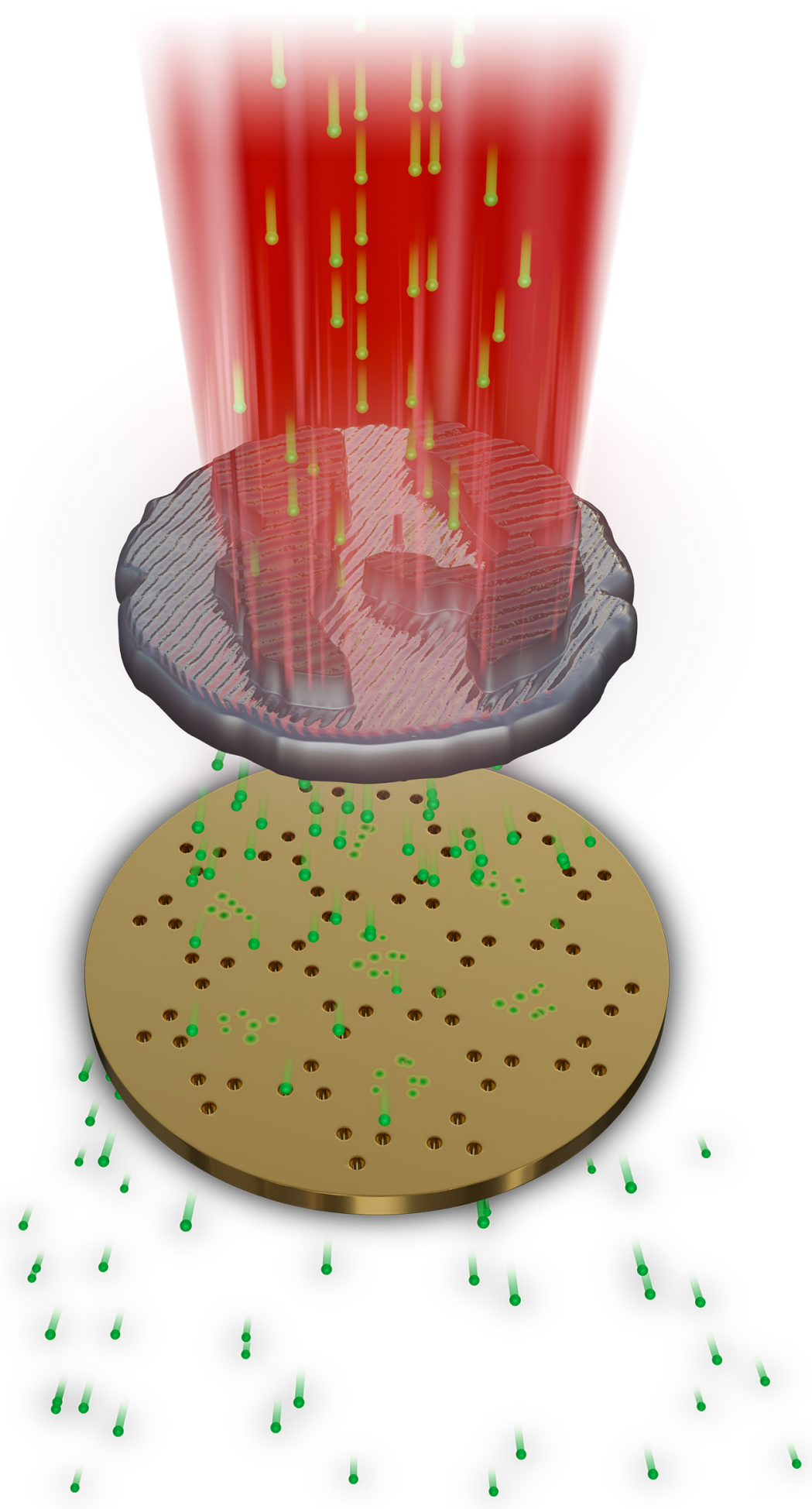

Artist's impression of charge-density wave domains imaged in the Göttingen Ultrafast Transmission Electron Microscope. Credit: Florian Sterl (Sterltech Optics). 



\section{Abstract}

High hopes are placed on materials with tunable properties and excitations for future applications in energy conversion devices. Functionality of devices, however, not only arises from the properties of individual materials but also from their interplay and nanoscale structuring. While a number of established experimental techniques are capable of tracking electronic excitations on femtosecond time and nanometer length scales, no time-resolved nanoimaging of a structural order parameter had previously been reported.

Addressing this challenge, the present cumulative thesis reports on the development and application of a time-resolved dark-field electron microscopy scheme implemented at the Göttingen Ultrafast Transmission Electron Microscope (UTEM). This nanoimaging approach combines femtosecond temporal and $5 \mathrm{~nm}$ spatial resolution with sensitivity to the structural component of a charge-density wave phase transition in $1 T$-polytype tantalum disulfide. Ultrashort laser pulses locally induce the phase transition, while the subsequent spatiotemporal relaxation dynamics of the structural order parameter is tracked using ultrashort electron pulses. Order parameter sensitivity is obtained by means of a dark-field aperture array, tailored to filter the periodicities of the charge-density wave in the diffraction plane of the microscope.

In the first publication contributing to this thesis, the preparation technique for the thin films of tantalum disulfide is introduced. Specimens obtained by ultramicrotomy are ideal for electron and $\mathrm{x}$-ray experiments in a transmission geometry, as exemplified by the investigation of manganeseand iron-intercalated tantalum disulfide. Static optical microscopy, electron diffraction and x-ray magnetic circular dichroism measurements serve to characterize these ferromagnetic thin films and to verify that the properties reflect those of the bulk crystals.

The second article describes the implementation of the ultrafast nanoimaging approach. A central aspect of the experiment is the design of a specimen that spatially structures the optical excitation pattern and allows for stroboscopic probing of the phase transition in tantalum disulfide at hundreds of kilohertz repetition rates. Based on parameters extracted from a steady-state heating experiment, the optically induced evolution of nanoscale charge-density wave domains in the free-standing thin film is reproduced in time-dependent Ginzburg-Landau simulations.

Finally, perspectives for future nanoimaging experiments are discussed. Allowing for sensitivity to further structural degrees of freedom in complex materials, ultrafast dark-field imaging will contribute to a better understanding of actively controlled processes in energy conversion devices. 


\section{Zusammenfassung}

Große Hoffnungen für zukünftige Anwendungen im Gebiet der Energieumwandlung werden auf Materialien mit abstimmbaren Eigenschaften und Anregungen gesetzt. Die Funktionalität miniaturisierter Systeme ergibt sich jedoch nicht nur aus den Eigenschaften der einzelnen Materialien, sondern auch aus deren Zusammenspiel und nanoskaliger Strukturierung. Während eine Reihe etablierter experimenteller Techniken in der Lage ist, elektronische Anregungen auf Femtosekunden-Zeit- und Nanometer-Längenskalen zu verfolgen, wurde bisher über keine zeitaufgelöste Nano-Abbildung eines strukturellen Ordnungsparameters berichtet.

Die vorliegende kumulative Dissertation behandelt die Entwicklung zeitaufgelöster Dunkelfeld-Bildgebung am Ultraschnellen Transmissions-Elektronenmikroskop (UTEM) in Göttingen. Dieser Ansatz kombiniert Femtosekunden-Zeitauflösung und eine räumliche Auflösung von $5 \mathrm{~nm}$ mit einer Empfindlichkeit für die strukturelle Komponente eines LadungsdichtewellenPhasenübergangs im 1T-Polytyp des Materials Tantaldisulfid. Ultrakurze Laserpulse induzieren lokal den Phasenübergang, während die raumzeitliche Relaxationsdynamik des strukturellen Ordnungsparameters mit ultrakurzen Elektronenpulsen verfolgt wird. Die Empfindlichkeit für den Ordnungsparameter wird mithilfe einer komplexen Dunkelfeld-Apertur erreicht.

In einer ersten Veröffentlichung wird die Technik zur Präparation der dünnen Schichten aus Tantaldisulfid vorgestellt. Die durch Ultramikrotomie gewonnenen Proben sind ideal für Elektronen- und Röntgenexperimente in einer Transmissionsgeometrie, wie die exemplarische Untersuchung von mit Mangan und Eisen interkaliertem Tantaldisulfid zeigt. Statische optische Mikroskopie, Elektronenbeugung und Messungen des zirkularen magnetischen Röntgendichroismus dienen dazu, diese ferromagnetischen Dünnschichten zu charakterisieren und zu bestätigen, dass ihre Eigenschaften denen der ursprünglichen Kristalle entsprechen.

Ein zweiter Artikel beschreibt die Umsetzung der zeitaufgelösten Nano-Abbildung. Ein zentraler Aspekt des Experiments ist die Herstellung einer Probe, die das optische Anregungsprofil räumlich strukturiert und gleichzeitig eine stroboskopische Untersuchung des Phasenübergangs in Tantaldisulfid bei Wiederholraten von hunderten Kilohertz ermöglicht. Basierend auf Parametern, die in einem stationären Heizexperiment gewonnen wurden, kann das Verhalten von nanoskaligen Ladungsdichtewellen-Domänen in der freistehenden Dünnschicht in zeitabhängigen Ginzburg-Landau-Simulationen reproduziert werden.

Abschließend werden Perspektiven für zukünftige Experimente auf Basis des vorgestellten Ansatzes diskutiert. Ultraschnelle Dunkelfeld-Bildgebung ermöglicht eine Empfindlichkeit auch für weitere strukturelle Freiheitsgrade in komplexen Materialien und wird so zu einem besseren Verständnis aktiv kontrollierter Prozesse auf dem Gebiet der Energieumwandlung beitragen. 


\section{Contents}

List of Figures $\quad$ xi

List of Tables $\quad$ xiii

List of Movies $\quad$ XV

List of Abbreviations and Symbols $\quad$ xvii

1 Introduction 1

2 Fundamentals of ultrafast transmission electron microscopy 7

2.1 Conventional transmission electron microscopy . . . . . . . . . . . . 8

2.2 Image contrast . . . . . . . . . . . . . . . . . . . . . . . 10

2.3 Elastic scattering . . . . . . . . . . . . . . . . 13

2.4 Specimen preparation . . . . . . . . . . . . . . . . . . 14

2.5 Specimen thickness measurements . . . . . . . . . . . . . . 17

2.6 Electron-sensitive detectors . . . . . . . . . . . . . . . . . . . 18

2.7 Ultrafast transmission electron microscopy . . . . . . . . . . . . . . . . . 20

2.8 Ultrafast imaging of structural and electronic dynamics . . . . . . . . . . . 23

3 Properties of transition metal dichalcogenides 29

3.1 Charge-density wave formation and metal-insulator transitions . . . . . . . . 32

3.2 Charge-density wave signatures in electron diffraction . . . . . . . . . . . . 35

3.3 Order parameters and phenomenological Landau theory . . . . . . . . . . . . 36

3.4 Equilibrium phase diagram of $1 T-\mathrm{TaS}_{2} \ldots \ldots \ldots \ldots$. . . . . . . . . 40

3.5 Ultrafast charge-density wave dynamics in $1 T-\mathrm{TaS}_{2} \ldots \ldots$. . . . . . . . 44

3.6 Kinematic diffraction simulation of the $\mathrm{NC}$ phase in $1 T-\mathrm{TaS}_{2} \ldots \ldots$. . . . . . 49

3.7 Intercalated TMDCs . . . . . . . . . . . . . . . . 50 
3.8 Properties of $\mathrm{Mn}_{0.25} \mathrm{TaS}_{2}$ and $\mathrm{Fe}_{0.25} \mathrm{TaS}_{2} \ldots \ldots \ldots \ldots \ldots$

4 Structural and magnetic characterization of large area, free-standing thin films of magnetic ion intercalated dichalcogenides $\mathrm{Mn}_{0.25} \mathrm{TaS}_{2}$ and $\mathrm{Fe}_{0.25} \mathrm{TaS}_{2} \quad 55$

4.1 Introduction . . . . . . . . . . . . . . . . 56

4.2 Material description . . . . . . . . . . . . . . . . 57

4.3 Preparation of free-standing thin films . . . . . . . . . . . . . 58

4.4 Characterization . . . . . . . . . . . . . . . . . . . . 59

4.4 Optical microscopy . . . . . . . . . . . . . . . 59

4.4.2 Transmission electron diffraction . . . . . . . . . . . . 60

4.4 .3 XMCD spectroscopy . . . . . . . . . . . . . . . . 61

4.5 Conclusion and outlook . . . . . . . . . . . . . . . . . 63

5 Ultrafast nanoimaging of the order parameter in a structural phase transition $\quad 67$

5.1 Main Text . . . . . . . . . . . . . . . . . . . 68

5.2 Materials and Methods . . . . . . . . . . . . . 76

5.2.1 Ultrafast transmission electron microscopy in DF mode . . . . . . . . 76

5.2.2 Manufacturing of DF aperture array and theoretical resolution limit . . 76

5.2.3 Specimen preparation and characterization . . . . . . . . . . 77

5.2.4 Image post-processing, image segmentation, and delay curves . . . . . 77

5.2.5 Extraction of excitation profile from experimental data . . . . . . . 78

5.2.6 Kinematical diffraction simulation of the $\mathrm{NC}$ phase . . . . . . . . . 78

5.2.7 Finite element simulations: General setup . . . . . . . . . . . . . 79

5.2.8 Finite element simulations: Field calculations . . . . . . . . . . . 80

5.2.9 Finite element simulations: Steady-state experiment . . . . . . . . 80

5.2.10 Finite element simulations: Heat transfer in ultrafast experiment . . . . 81

5.2.11 Finite element simulations: Time-dependent Ginzburg-Landau approach 82

5.3 Supplementary Text . . . . . . . . . . . . . . . . . . . 84

5.3.1 Structured excitation profile inside the circular gold aperture . . . . . . 84

5.3.2 Orientation of NC/IC phase boundaries along preferential directions . . 85

5.4 Supplementary Figures . . . . . . . . . . . . . . . . . 86

5.5 Supplementary Tables . . . . . . . . . . . . . . . . . . . 95

5.6 Supplementary Movies . . . . . . . . . . . . . . . . . 96 
6 Discussion $\quad 99$

6.1 Preparation and characterization of thin films for UTEM experiments . . . . . . 100

6.1.1 Preparation of thin films using ultramicrotomy . . . . . . . . . . . . 100

6.1.2 Specimen thickness measurements . . . . . . . . . . . . . . . 101

6.1.3 Further specimen characterization . . . . . . . . . . . . . . 102

6.1.4 A broader perspective on the dichroic probing of magnetism . . . . . 103

6.1.5 Design of the specimen structure for the DF experiment . . . . . . . 105

6.1.6 Alternate routes to high-repetition-rate probing . . . . . . . . . . . 106

6.2 Ultrafast nanoimaging using UTEM . . . . . . . . . . . . . . 110

6.2.1 Feasibility of the DF imaging technique . . . . . . . . . . . . 110

6.2.2 DF imaging and electron beam coherence . . . . . . . . . . . . . . 111

6.2.3 The role of probabilistic domain switching in the NC phase . . . . . . 113

6.2.4 A note on CDW phases and domain walls . . . . . . . . . . . . . 115

6.2.5 Ultrafast nanoimaging of electronic order parameters . . . . . . . . . 116

6.2.6 Ultrafast DF imaging and 4D-STEM . . . . . . . . . . . . . . . . . 119

6.2.7 Perspectives for future nanoimaging experiments . . . . . . . . . 121

6.3 Concluding remarks . . . . . . . . . . . . . . . . 123

$\begin{array}{lll}\text { A High-resolution prints of diffractograms } & 127\end{array}$

$\begin{array}{ll}\text { Bibliography } & 131\end{array}$

Author contributions, publications, conferences and awards $\quad 175$

$\begin{array}{ll}\text { Danksagung } & 179\end{array}$ 



\section{List of Figures}

Figure 1.1 Active control of material properties using terahertz and optical pulses _ . . 3

Figure 1.2 Experimental techniques allowing for spatiotemporal imaging of ultrafast dynamics . . . . . . . . . . . . . . . . . . . 4 4

Figure 2.1 Schematic setup and electron pulse properties of the Göttingen UTEM in-

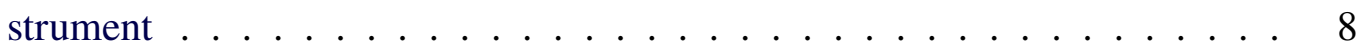

Figure 2.2 Schematic of a TEM and its basic operation modes . . . . . . . . . . . . . 9

Figure 2.3 Image formation and contrast transfer channels in a TEM . . . . . . . . . . 11

Figure 2.4 Specimen preparation using ultramicrotomy . . . . . . . . . . . . . 15

Figure 2.5 Light microscopy images of ultramicrotomed graphite films on a grid . . . . 16

Figure 2.6 Exemplary experimental results achievable with the current status of the Göttingen UTEM instrument . . . . . . . . . . . . . . . . . . . 22

Figure 2.7 Ultrafast TEM images and diffraction patterns of $\mathrm{VO}_{2} \ldots \ldots$. . . . . . . 25

Figure 2.8 Single-nanoparticle morphology dynamics . . . . . . . . . . . . . . . 26

Figure 2.9 Imaging of acoustic phonon propagation in multilayer $1 T-\mathrm{TaS}_{2} \ldots \ldots$

Figure 3.1 Crystal structure of TMDC polytypes . . . . . . . . . . . . . . . . 30

Figure 3.2 Coherent manipulation of valley pseudospin in $\mathrm{WSe}_{2} \ldots \ldots$. . . . . . . . 31

Figure 3.3 Peierls instability in a one-dimensional chain of atoms . . . . . . . . . . . 33

Figure 3.4 Fermi surface nesting in one- and two-dimensional cases . . . . . . . . . . 34

Figure 3.5 Landau free-energy densities for the Ising model and a complex order parameter 38

Figure 3.6 Phase diagram, real-space structure and reciprocal structure of the CDW phases in $1 T-\mathrm{TaS}_{2} \ldots \ldots \ldots \ldots \ldots$. . . . . . . . . . . . . . . . .

Figure 3.7 Static angle-resolved photoemission spectra of the C and IC phase in $1 T-\mathrm{TaS}_{2} 42$

Figure 3.8 Electrical resistivity and core-level splitting in $1 T-\mathrm{TaS}_{2} \ldots \ldots$. . . . . . . 43

Figure 3.9 Electronic dynamics in $1 T-\mathrm{TaS}_{2}$ after an optical CDW quench . . . . . . . . 45

Figure 3.10 Structural dynamics in $1 T-\mathrm{TaS}_{2}$ after an optical CDW quench . . . . . . . 46 
Figure 3.11 Ultrafast NC CDW/PLD quench in $1 T-\mathrm{TaS}_{2}$ observed by UED $\ldots . . . .47$

Figure 3.12 Supercell for "commensurate" NC CDW in $1 T-\mathrm{TaS}_{2} \ldots \ldots$. . . . . . . . . 49

Figure 4.1 Crystal structure and optical microscopy images of intercalated TMDC samples 56

Figure 4.2 Selected area electron diffraction images of intercalated TMDC samples . . 60

Figure 4.3 X-ray absorption spectra and XMCD spectra of intercalated TMDC samples 61

Figure 4.4 Hysteresis curves at the $L_{3}$ edge of manganese and iron . . . . . . . . . . 63

Figure 5.1 Dark-field imaging in the ultrafast transmission electron microscope . . . . 69

Figure 5.2 Ultrafast dark-field domain imaging of charge-density wave dynamics . . . . 71

Figure 5.3 Dark-field domain imaging with continuous-wave laser excitation . . . . . . 72

Figure 5.4 Time-resolved Ginzburg-Landau simulations of optically induced order parameter dynamics . . . . . . . . . . . . . . . . . . . . . 74

Figure 5.5 Diffraction pattern of the IC phase of $1 T-\mathrm{TaS}_{2} \ldots \ldots \ldots$

Figure 5.6 Extended schematic of the experiment and the DF aperture array . . . . . 87

Figure 5.7 Ultrafast DF images as a function of laser fluence at fixed pump/probe delays 88

Figure 5.8 Demonstration of image segmentation f . . . . . . . . . . . . . . . . . . . 89

Figure 5.9 Imaging of the specimen structure with a continuous electron beam . . . . . 90

Figure 5.10 Order parameter trajectories on the free-energy surface for different levels of local excitation . . . . . . . . . . . . . . . . . . 991

Figure 5.11 Domain wall width and extended simulation results $\ldots \ldots$. . . . . . 92

Figure 5.12 Ultrafast DF images as a function of linear pump polarization . . . . . . . . 93

Figure 5.13 Identification of reciprocal lattice directions in the steady-state DF images . 94

Figure 6.1 Application of electron vortex beams to EMCD . . . . . . . . . . . . . 104

Figure 6.2 Transfer of a $1 T-\mathrm{TaS}_{2}$ film using FIB manipulation . . . . . . . . . . . . . 107

Figure 6.3 Crystallization of FIB-deposited platinum . . . . . . . . . . . . . . 107

Figure 6.4 TEM images and diffraction patterns of polycrystalline diamond membranes 109

Figure 6.5 Determination of CDW spot width and DF aperture diameter . . . . . . . 112

Figure 6.6 Single-pulse domain switching in the NC phase of $1 T-\mathrm{TaS}_{2} \ldots \ldots$. . . . 114

Figure 6.7 Two-color PINEM probing of electronic dynamics in $\mathrm{VO}_{2} \ldots \ldots$. . . . . . 117

Figure 6.8 Scanning near-field optical microscopy of electronic dynamics in $\mathrm{VO}_{2}$. . . 118

Figure 6.9 Proposal of $\mathrm{DF}$ apertures for polycrystalline $\mathrm{VO}_{2} \ldots \ldots \ldots$. . . . . . . 121 


\section{List of Tables}

Table 3.1 Coefficients for atomic scattering factors of sulfur and tantalum . . . . . . . 50

Table 3.2 Properties of $\mathrm{Mn}_{0.25} \mathrm{TaS}_{2}$ and $\mathrm{Fe}_{0.25} \mathrm{TaS}_{2} \ldots \ldots \ldots \ldots$. . . . . . . 52

Table 5.1 Physical material properties used in the finite element simulations . . . . . . 95

Table 6.1 Comparison of calculated MFP values for gold, silicon nitride and $1 T-\mathrm{TaS}_{2} \ldots 102$

Table 6.2 Thermal conductivities of TEM membranes (in-plane) and bulk materials . . . 108 



\section{List of Movies}

Movie 5.1 Ultrafast DF image series . . . . . . . . . . . . . . . . . 96

Movie 5.2 Ultrafast bright-field $(\mathrm{BF})$ image series . . . . . . . . . . . . . . . 96

Movie 5.3 Steady-state DF image series . . . . . . . . . . . . . . . . 96 96

Movie 5.4 Time-dependent Ginzburg-Landau simulation of ultrafast DF images . . . . . 96

Movie 5.5 Time-dependent Ginzburg-Landau simulation (non-equilibrium contribution) 96 



\section{List of Abbreviations and Symbols}

\section{Abbreviations}

\begin{tabular}{ll} 
Abbreviation & Description \\
\hline (tr-)ARPES & (Time- and) angle-resolved photoemission spectroscopy \\
BF & Bright-field \\
BFP & Back-focal plane \\
C & Commensurate \\
CCD & Charge-coupled device \\
CDW & Charge-density wave \\
CMOS & Complementary metal-oxide semiconductor \\
CTF & Contrast transfer function \\
CVD & Chemical vapor deposition \\
DF & Dark-field \\
DQE & Detective quantum efficiency \\
EELS & Electron energy loss spectroscopy \\
EFTEM & Energy-filtered transmission electron microscopy \\
EMCD & Electron energy-loss magnetic chiral dichroism \\
FIB & Focused ion beam \\
FWHM & Full width at half maximum \\
FY & Fluorescence yield \\
IC & Incommensurate \\
LEEM & Low-energy electron microscopy (or microscope) \\
MFP & Mean free path \\
MIT & Metal-insulator transition \\
MPMS & Magnetic property measurement system \\
NC & Nearly commensurate \\
&
\end{tabular}




\begin{tabular}{ll} 
Abbreviation & Description \\
\hline PEEM & Photoemission electron microscopy (or microscope) \\
PINEM & Photon-induced near-field electron microscopy \\
PLD & Periodic lattice distortion \\
RT & Room temperature \\
SAED & Selected area electron diffraction \\
SNOM & Scanning near-field optical microscopy (or microscope) \\
SNR & Signal-to-noise ratio \\
STEM & Scanning transmission electron microscopy (or microscope) \\
STM & Scanning tunneling microscopy (or microscope) \\
TEM & Transmission electron microscopy (or microscope) \\
TEY & Total electron yield \\
TMDC & Transition metal dichalcogenide \\
UED & Ultrafast electron diffraction \\
UTEM & Ultrafast transmission electron microscopy (or microscope) \\
XAS & X-ray absorption spectroscopy \\
XMCD & X-ray magnetic circular dichroism \\
XUV & Extreme ultraviolet \\
ZLP & Zero-loss peak
\end{tabular}

\section{Latin symbols}

\begin{tabular}{ll} 
Symbol & Description \\
\hline$a$ & Crystal lattice constant (norm of $\left.\vec{a}_{1}\right)$ \\
$\vec{a}_{i}$ & Crystal lattice vectors $(i \in[1,3])$ \\
$\vec{A}$ & Amplitude of PLD \\
$b$ & Crystal lattice constant (norm of $\left.\vec{a}_{2}\right)$ \\
$\vec{b}_{i}$ & Reciprocal lattice vectors $(i \in[1,3])$ \\
$c$ & Crystal lattice constant (norm of $\left.\vec{a}_{3}\right)$ \\
$d, d_{h k l}$ & Distance [between adjacent $(h k l)$ lattice planes] \\
$E, E_{\mathrm{F}}$ & (Fermi) energy \\
$f$ & Atomic scattering factor \\
$F, F_{h k l}$ & Structure factor [of a Bragg reflection $(h k l)]$ \\
& or Landau free-energy density
\end{tabular}




\begin{tabular}{ll} 
Symbol & Description \\
\hline$I, I_{h k l}$ & Intensity [of a Bragg reflection $(h k l)]$ \\
$\vec{k}, \vec{k}_{\mathrm{F}}$ & (Fermi) wave vector (crystallographic definition with $|\vec{k}|=1 / \lambda)$ \\
$\vec{q}, \vec{q}_{h k l}$ & Scattering vector [of a Bragg reflection $(h k l)]$ \\
& $\begin{array}{l}\left.\text { (crystallographic definition with }\left|\vec{q}_{h k l}\right|=1 / d_{h k l}\right) \\
\vec{Q}_{i}\end{array}$ \\
$\vec{r}$ & Wave vector of CDW (crystallographic definition with $\left.\left|\vec{Q}_{i}\right|=1 / \lambda\right)$ \\
$t$ & Position vector \\
& Time \\
$\Delta t$ & or Specimen thickness \\
$T$ & Temporal delay \\
$T_{\mathrm{RT}}$ & Temperature \\
$T_{\mathrm{el}}, T_{\mathrm{ph}}$ & Room temperature \\
$T^{*}$ & Electron/lattice temperature \\
$T_{\mathrm{C}}$ & Phase transition temperature \\
& Critical temperature (low-temperature side of the hysteresis loop) \\
$T_{1}$ & or Curie temperature \\
$\vec{u}$ & High-temperature side of the hysteresis loop \\
$Z$ & Atomic displacement of PLD \\
& Atomic number
\end{tabular}

\section{Greek symbols}

\begin{tabular}{ll} 
Symbol & Description \\
\hline$\alpha_{0}$ & Parameter for Landau free-energy density (quadratic term) \\
$\beta$ & Parameter for Landau free-energy density (quartic term) \\
$\gamma$ & Parameter for Landau free-energy density (sextic term) \\
$\theta$ & Scattering semi-angle \\
$\lambda$ & Electron/photon/CDW wavelength \\
& or Mean free path \\
$\rho$ & Density \\
$\phi, \phi_{\text {eq }}$ & Scalar order parameter (at free-energy minimum) \\
$\varphi$ & Phase \\
$\psi$ & Electron wave function \\
$\omega$ & Angular frequency
\end{tabular}





\section{Chapter 1}

\section{Introduction}

In the light of global climate change, the development of efficient energy conversion and storage strategies is more important than ever before. These are applied, among others, in solar cells, artificial photosynthesis and the design of high-density batteries (1-4). On a microscopic scale, energy conversion happens in complex multi-step processes involving electronic, structural and magnetic degrees of freedom in a material. If these interactions are not sufficiently controlled, conversion efficiencies of optical excitations, for example in solar cells, remain below their theoretical limits and dissipation occurs (3). Therefore, a fundamental understanding of energy conversion processes is essential for a sustainable future.

High hopes are placed on materials with tunable properties and phases. The classical phase diagram describes the different phases of a material as a function of temperature, pressure or composition. For the case of tunable materials, phases characterized by completely new properties appear along the axes of the phase diagram, typically due to correlations between multiple degrees of freedom. A material class that displays an especially rich phase diagram and promises a high degree of tunability in energy conversion applications are transition metal dichalcogenides (TMDCs).

Depending on their composition and temperature, these layered quasi-two-dimensional materials can be insulating, semiconducting or metallic $(5,6)$. Under the influence of pressure or doping, superconducting states are observed $(7,8)$, and the phase diagram is further modified as a function of specimen thickness (9). Additionally, the layered structure facilitates interlayer shear displacements (10) and intercalation, i.e., the introduction of foreign atom species between the layers that modify structural, electronic or magnetic material properties (11). Last but not least, the reduced dimensionality leads to a spatial modulation of the charge density in a wide range of TMDCs - the formation of a charge-density wave (CDW) (12). 
Therefore, TMDCs are a prime example of a material class with tunable structural, electronic and magnetic properties. When extending the phase diagram into two additional dimensions, photodoping and time, the dynamic response of a material to specific optical stimuli can be described (13). This is particularly interesting because recent experiments found a number of metastable phases in TMDCs that are only accessible via non-equilibrium pathways (14-17). Therefore, active control over the temporal system response $(18,19)$ is of utmost importance when a material is used in energy conversion applications. Different approaches to this kind of control have been demonstrated in a large number of experiments (20-25). Recently, topological properties of the TMDC WTe 2 and the related transition metal trichalcogenide $\mathrm{LaTe}_{3}$ have been exploited in ultrafast electron diffraction (UED) experiments $(10,26)$.

The stable modification of $\mathrm{WTe}_{2}$ occurs in a crystal structure that is distorted with respect to other TMDC representatives, leading to the absence of inversion symmetry. This gives rise to the appearance of chiral pairs of topological Weyl points in the dispersion relation of the material, whose presence can be experimentally verified by chiral magnetic effects in the bulk and topological Fermi arcs on the surface (27). SiE et al. have shown that terahertz pulses can be used to unidirectionally drive the distorted phase towards a centrosymmetric structure (Fig. 1.1A and B). In the process, Weyl nodes of opposite chirality annihilate to form an energetically degenerate Dirac point, transiently suppressing the topological phase of $\mathrm{WTe}_{2}(10,28)$.

As a consequence of a specific two-dimensional topology of the Fermi surface and strong electron-phonon coupling, the layered compound $\mathrm{LaTe}_{3}$ forms an unidirectional CDW in its ground state. A second CDW along the perpendicular direction is only suppressed by a slight in-plane anisotropy of the lattice constants. Using an ultrashort optical stimulus, KogAR et al. were able to transiently lift the suppression of the competing CDW order during the first few picoseconds after optical excitation (Fig. 1.1C), thus giving rise to a transient phase with no equilibrium counterpart $(26,29)$.

In these particular examples, optical control aims at accessing and modifying intrinsic properties of a single material. However, when incorporated in future devices, functionality not only arises from bulk properties, but also from a combination of materials in heterostructures (30, 31). For example, this allows for designing charge-separation pathways across interfaces as required in solar cells and photocatalytic water splitting (32-35). Experimental techniques that can spatially resolve this kind of dynamics on its intrinsic femto- to nanosecond time scales are therefore urgently needed.

Various experimental techniques fundamentally allow for combining the femtosecond time resolution of stroboscopic pump/probe approaches with nanometer spatial resolution, some of 
A

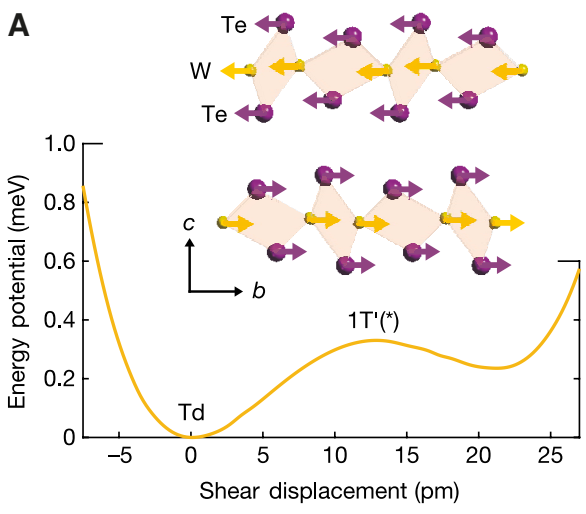

B

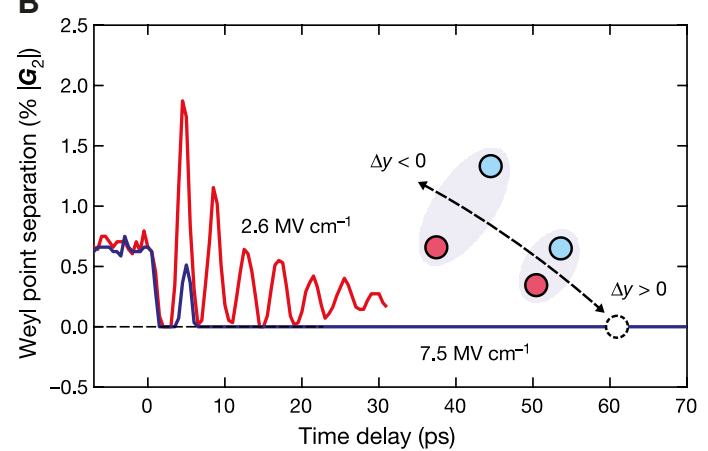

C

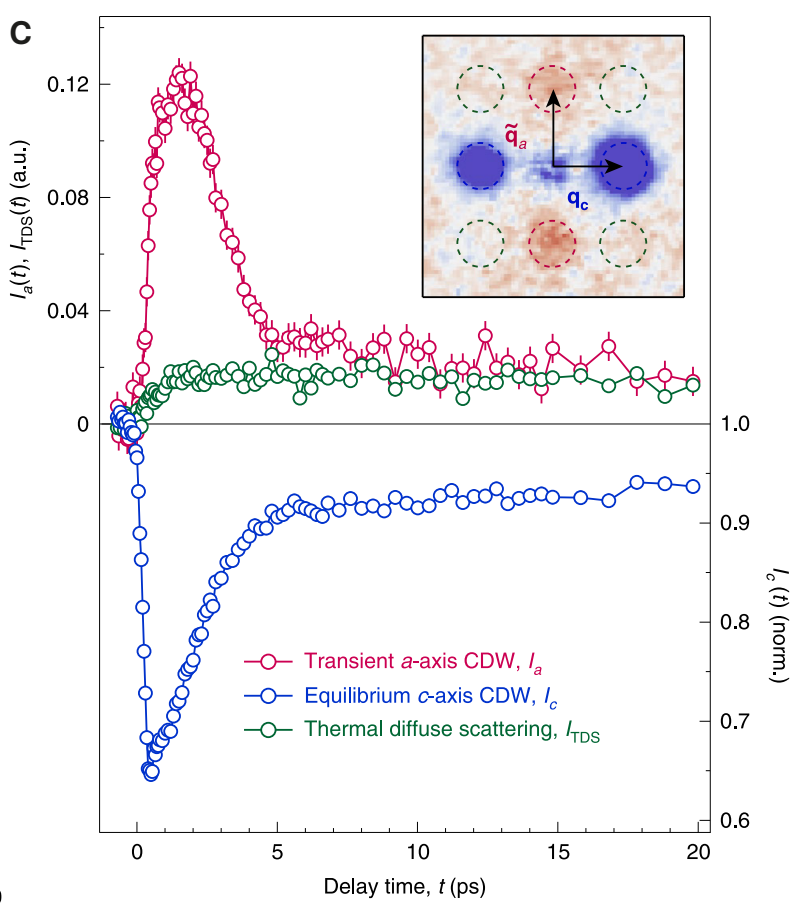

Figure 1.1: Active control of material properties using terahertz and optical pulses. (A) The TMDC WTe consists of $\mathrm{Te}-\mathrm{W}-\mathrm{Te}$ trilayers that are bound by weak van-der-Waals forces along the crystallographic $c$-axis. Terahertz excitation drives the orthorhombic $T d$ ground state towards the centrosymmetric $1 T^{\prime}\left(^{*}\right)$ phase by interlayer shearing (top). The energy potential corresponding to this process is depicted schematically (bottom). (B) The interlayer shear displacement $\Delta y$ influences the separation of Weyl points of opposite chirality (red and blue circles) in $\mathrm{WTe}_{2}$. The separation is shown as a function of time in units of the reciprocal lattice vector $\left|\mathbf{G}_{2}\right|$ for two different terahertz field strengths. While oscillations of the Weyl point separation are observed for $2.6 \mathrm{MV} / \mathrm{cm}$, a long-lived state is reached after excitation using $7.5 \mathrm{MV} / \mathrm{cm}$ field strength. In this state, pairs of Weyl points have annihilated to form Dirac points (dashed circle) in an inversion-symmetric band structure. A and B reprinted by permission from Springer Nature Customer Service Centre GmbH: Springer Nature, Nature, Ref. (10), Copyright 2019 Springer Nature Limited. (C) Time traces of the equilibrium CDW along the $c$-axis $\left(I_{c}\right)$, the transient $a$-axis CDW $\left(I_{a}\right)$ and the diffuse background scattering intensity $\left(I_{\mathrm{TDS}}\right)$ after optical excitation of $\mathrm{LaTe}_{3}$. In contrast to the crystal structure of TMDCs, the crystallographic $b$-axis of $\mathrm{LaTe}_{3}$ is perpendicular to the layers, while the $a$ - and $c$-directions are in-plane. Inset: The amplitudes of the equilibrium CDW and the optically induced CDW can be separately extracted from diffraction patterns due to their perpendicular scattering vectors $\mathbf{q}_{\mathbf{c}}$ and $\tilde{\mathbf{q}}_{\mathbf{a}}$. Dashed circles mark the integration areas of the time traces. Reprinted by permission from Springer Nature Customer Service Centre GmbH: Springer Nature, Nature Physics, Ref. (26), Copyright 2019 The Author(s), under exclusive licence to Springer Nature Limited. 
A

Electron microscopy

Electron probe
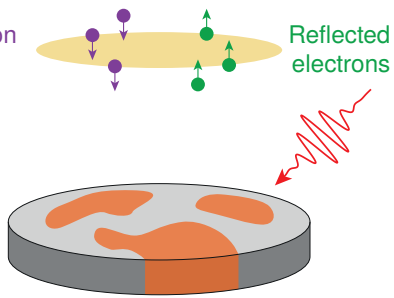

Trans-

mitted

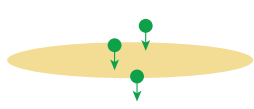

Electron optics
B

Photoemission

electron microcopy

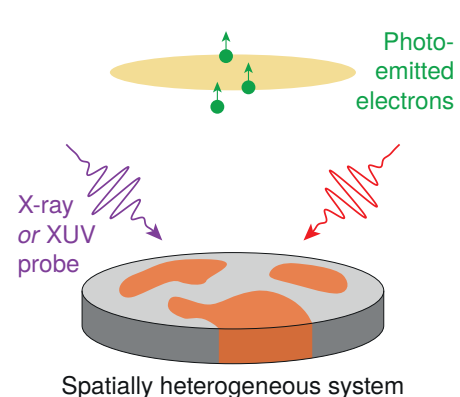

C Scanning probe microscopy

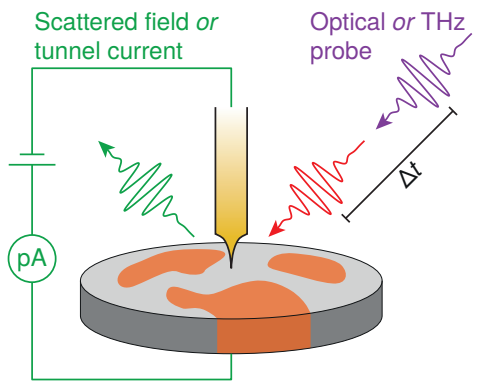

Optical pump pulse

Probe pulse

Probe response

Figure 1.2: Experimental techniques allowing for spatiotemporal imaging of ultrafast dynamics. (A) Ultrafast electron microscopy uses electron optics to illuminate the specimen using an ultrashort electron pulse. The transmitted or reflected electrons are used to form an image on an electron-sensitive detector. (B) Time-resolved photoemission electron microscopy uses an X-ray or extreme ultraviolet (XUV) pulse to photoemit electrons from the specimen. Electron optics are used to collect the electrons and form an image on a detector. (C) Timeresolved scanning probe techniques take advantage of the localization of optical or terahertz pulses at the apex of a nanometric tip. By scanning the tip over the surface, variations in scattered field or tunnel current can be used to construct an image of the specimen.

which are schematically depicted in Fig. 1.2. An ultrashort optical pump pulse (red) excites spatiotemporal dynamics in a heterogeneous system. Different pulsed probe interactions (purple) are used to measure a spatially resolved specimen response (green) as a function of temporal delay $\Delta t$ between pump and probe. Except for transmission electron microscopy (TEM ${ }^{1}$; panel A), which is a bulk probe, these are surface-sensitive techniques. Often, transitions between different approaches are gradual, such that some low-energy electron microscopes (LEEM; panel A) may also be used as photoemission electron microscopes (PEEM; panel B) by switching the probe and collecting photoemitted instead of reflected electrons. Generally, electron optical systems can be designed to offer a great flexibility and deliver, besides spatial images, spectroscopic and reciprocal space information $(36,37)$. Scanning probe techniques (panel C) can be combined with a wide range of tip-sample interactions resulting in techniques such as scanning tunneling microscopy (STM) and scanning near-field optical microscopy (SNOM) $(38,39)$. By pulsing the STM tip bias using a terahertz field or by using a pulsed near-field for SNOM, both techniques can be brought into the time domain (40-44).

When imaging ultrafast spatiotemporal dynamics, it is useful to have access to a single observable that describes the state of the system as a function of position and time-an order

${ }^{1}$ In the case of this and other abbreviations for microscopy techniques, we use the same abbreviation for the microscopy technique and the microscope device itself. 
parameter. In the extreme case of a phase transition induced by the optical pump, the order parameter of the original phase vanishes while new order emerges. Scanning probe techniques have demonstrated ways to access electronic order parameters, for example by mapping the evolution of the free-carrier response during a metal-insulator phase transition (43-46). Timeand angle-resolved photoemission (tr-ARPES) is nowadays a "workhorse" technique that can directly visualize the underlying electronic band structure $(35,47-50)$, and the number of published time-resolved PEEM experiments testifies to the potential of combining space- and time-domain approaches $(32,51-55)$. On the structural side, which electron microscopy is intrinsically able to access, we find a wealth of examples for the spatiotemporal mapping of specimen morphology dynamics using ultrafast transmission electron microscopy (UTEM) (56-62), and for the investigation of spatially averaged structural order parameters in UED studies $(10,24-26,63-65)$, as exemplified above for the two cases of $\mathrm{WTe}_{2}$ and $\mathrm{LaTe}_{3}$. However, no time-resolved nanoimaging of a structural order parameter has previously been reported in the literature.

The present thesis contributes to this area of research by introducing a novel nanoimaging approach at the Göttingen Ultrafast Transmission Electron Microscope (66). This approach combines femtosecond temporal and nanometer spatial resolution with sensitivity to the structural component of a CDW phase transition in $1 T-\mathrm{TaS}_{2}$, a widely investigated TMDC representative. We use ultrashort laser pulses to locally induce the phase transition, and track the subsequent spatiotemporal relaxation dynamics of the order parameter using ultrashort electron pulses. The resulting movies represent the first real-space observation of transient domain formation in a charge-density wave system.

The scientific studies that constitute the central part of this thesis have been conducted as part of the Collaborative Research Center 1073 "Atomic scale control of energy conversion”, which aims at understanding and controlling the elementary steps of energy conversion in materials with tunable excitations and interactions.

\section{Outline}

This cumulative thesis is composed of two original publications in Chapters 4 and 5, both of which are independent works giving a short introduction into the topic, illustrating the experimental work and discussing the respective results. Chapters 2 and 3 are structured in such a way that they embed the two publications in a broader scientific context. 
A brief introduction into the UTEM method is given in Chapter 2. Starting from conventional TEM, we illustrate several theoretical and experimental basics that are of relevance to the following chapters. The chapter is closed by a short summary of pioneering UTEM works that have contributed to the developments presented in this thesis. Chapter 3 introduces the TMDC materials under investigation in both publications and summarizes the results of previous time-domain studies characterizing the materials' behavior.

Chapter 4 describes a mechanical technique to prepare x-ray- and electron-transparent thin films from bulk crystals. The article "Structural and magnetic characterization of large area, freestanding thin films of magnetic ion intercalated dichalcogenides $\mathrm{Mn}_{0.25} \mathrm{TaS}_{2}$ and $\mathrm{Fe}_{0.25} \mathrm{TaS}_{2}$ " [see Ref. (67)] illustrates how different experimental techniques were used to verify the integrity of the obtained films.

In Chapter 5, results of the first real-space imaging of a structural phase transition are presented. In the publication "Ultrafast nanoimaging of the order parameter in a structural phase transition" [see Ref. (68)], we outline how a tailored dark-field imaging approach visualizes the spatiotemporal evolution of structural domains in $1 T-\mathrm{TaS}_{2}$, making full use of the capabilities of the UTEM method. Furthermore, complementary numerical simulations offer intriguing insights into the ultrafast relaxation dynamics near domain walls.

Finally, the main results of the two publications are summarized in Chapter 6 and put together to form a comprehensive picture. We discuss details of the experimental approach and deduce perspectives for future real-space imaging experiments. 


\section{Chapter 2}

\section{Fundamentals of ultrafast transmission electron microscopy}

The advent of electron microscopy came with the works of Max KnOLL and ERnst Ruska in the 1920s in Berlin. After realizing that magnetic coils can be used to focus electron beams just as a convex optical lens focuses light rays, it did not take long until the first transmission electron microscope (TEM) was constructed in 1932 (69). Thanks to the shorter electron wavelength, early TEMs soon surpassed light microscopes in terms of image resolution (70).

Today, TEMs are an indispensable research tool in a broad range of disciplines investigating the structure of matter from sub-nanometer to micrometer length scales. In solid state physics and material sciences, the method enables the investigation of crystal structures and defects (71), the distribution of phases $(72,73)$, chemical reactions $(74,75)$ and electromagnetic fields $(76)$ on a single-atom scale. Extended by electron energy loss spectroscopy (EELS) or energy-dispersive xray spectroscopy (77), analytical TEMs can map the composition of a specimen (78), analyze the chemical state of elements (79) and even map the vibrational response of individual atoms (80). In the biological sciences, cryo-TEMs are increasingly used to determine the atomic structure of proteins (81), even supplanting the hitherto dominating x-ray crystallography approach (82).

The rapidly evolving ultrafast transmission electron microscopy (UTEM) extends the preceding capabilities by simultaneous temporal resolution on the femtosecond scale (83). This is realized by implementing a stroboscopic laser pump/electron probe scheme in the tradition of all-optical pump/probe techniques in the fields of femtochemistry and condensed matter physics (84-86). Ultrashort electron probe pulses are generated by photoemission from a suitable emitter. This is schematically depicted in Fig. 2.1 by the example of the Göttingen UTEM instrument. In this way, UTEM allows for unique access to ultrafast dynamics of electronic, structural and magnetic degrees of freedom in spatially heterogeneous systems.

The purpose of this chapter is to outline the fundamentals of conventional and ultrafast TEM as a basis for the descriptions concerning sample preparation and characterization (Chapter 4) and 
a

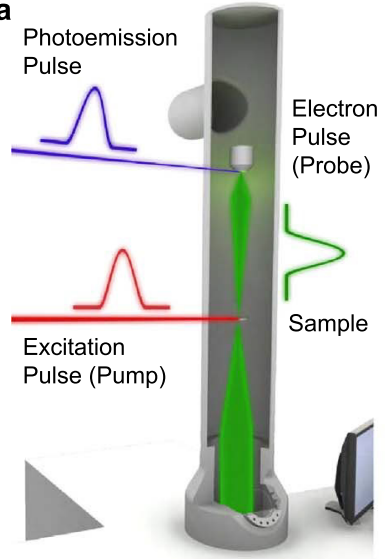

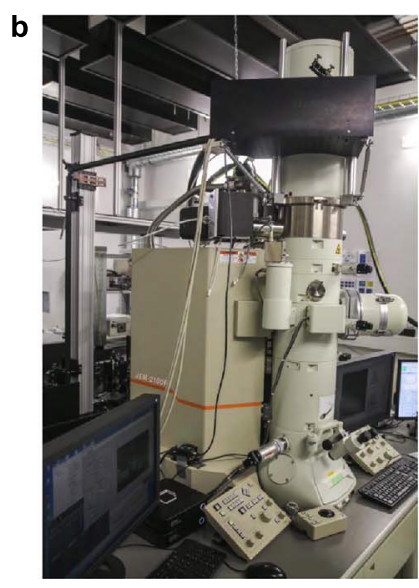
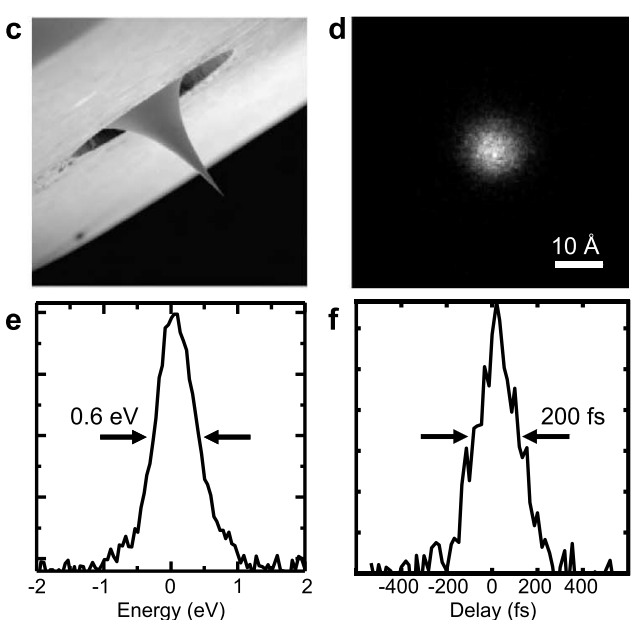

Figure 2.1: Schematic setup and electron pulse properties of the Göttingen UTEM instrument. A laserdriven Schottky field emission electron gun $(\mathbf{A})$ is combined with the column of a JEOL JEM-2100F (B). Side illumination of a nanoscopic $\mathrm{ZrO} / \mathrm{W}\left(\begin{array}{lll}1 & 0 & 0\end{array}\right)$ tip emitter $(\mathbf{C})$ enables the generation of ultrashort electron bunches, which can be focused down to $0.89 \mathrm{~nm}(\mathbf{D})$, with an energy width of $0.6 \mathrm{eV}$ (E) and a duration of $200 \mathrm{fs}(\mathbf{F})$ (apertured beam, at $200 \mathrm{kV}$ acceleration voltage). Figure and caption reprinted from Ref. (66).

the implementation of the ultrafast nanoimaging experiment at the Göttingen UTEM instrument (Chapter 5). The chapter is concluded by a concise discussion of prior approaches towards the spatiotemporal imaging of structural phase transitions using UTEM. Additionally, we will formulate the main challenges in this field that lead to the developments described in the two publications comprised by this thesis.

\subsection{Conventional transmission electron microscopy}

As first formulated by ERNST ABBE in 1873, the resolution of optical microscopes is fundamentally limited by half of the shortest wavelength in the visible range, i.e., limited to $200 \mathrm{~nm}$ (87). Given their wave nature, the same limit applies to electrons which are typically accelerated to energies between $80 \mathrm{keV}$ and $300 \mathrm{keV}$, corresponding to wavelengths of $4.18 \mathrm{pm}$ to $1.97 \mathrm{pm}$ (88). However, due to lens aberrations, the construction of TEMs that are able to reach the resolution limit is still subject of active research. The development of aberration correctors has pushed the absolute resolution below $50 \mathrm{pm}$ (at $300 \mathrm{keV}$ ) and the relative resolution to 15 times the electron wavelength (at $40 \mathrm{keV}$ ) (89-91). Apart from the involved length scales, the basic operating principles of TEMs and transmitted light microscopes are largely similar. A source emits a beam of light or electrons which is formed by condenser lenses to illuminate a specimen. Behind the specimen, a number of imaging lenses forms an image of the transmitted light or electrons on a detector. 
A simplified schematic of a TEM is shown in Fig. 2.2A. While early TEMs used a currentcarrying tungsten wire to generate free electrons via thermionic emission, similar to a light bulb, current implementations of TEMs use specialized emitters where electron emission is confined to a more point-like source. Subsequently, a series of electrostatic electrodes and an electrostatic accelerator form the beam and accelerate the electrons to the desired beam energy. The combination of emitter, electrostatic electrodes and accelerator is called the gun of the microscope. The electrostatic fields forms a gun cross-over at the exit aperture of the accelerator, where it is coupled into the column.

From here on, a number of magnetic condenser lenses and a circular condenser aperture allow for precise control over the beam diameter, the beam convergence angle and the beam current in the specimen plane. This is done by changing the focal length of the individual lenses, i.e., by adjusting the electric current flowing through the lens coils and by choice of aperture size. The specimen is inserted into the objective lens (OL) which is the strongest lens in the microscope. Typically, this lens is constructed symmetrically with respect to the specimen plane: it has a prefield taking part in forming the illuminating beam, and a postfield responsible for the first image magnification step leading to the formation of the first intermediate image. Between the

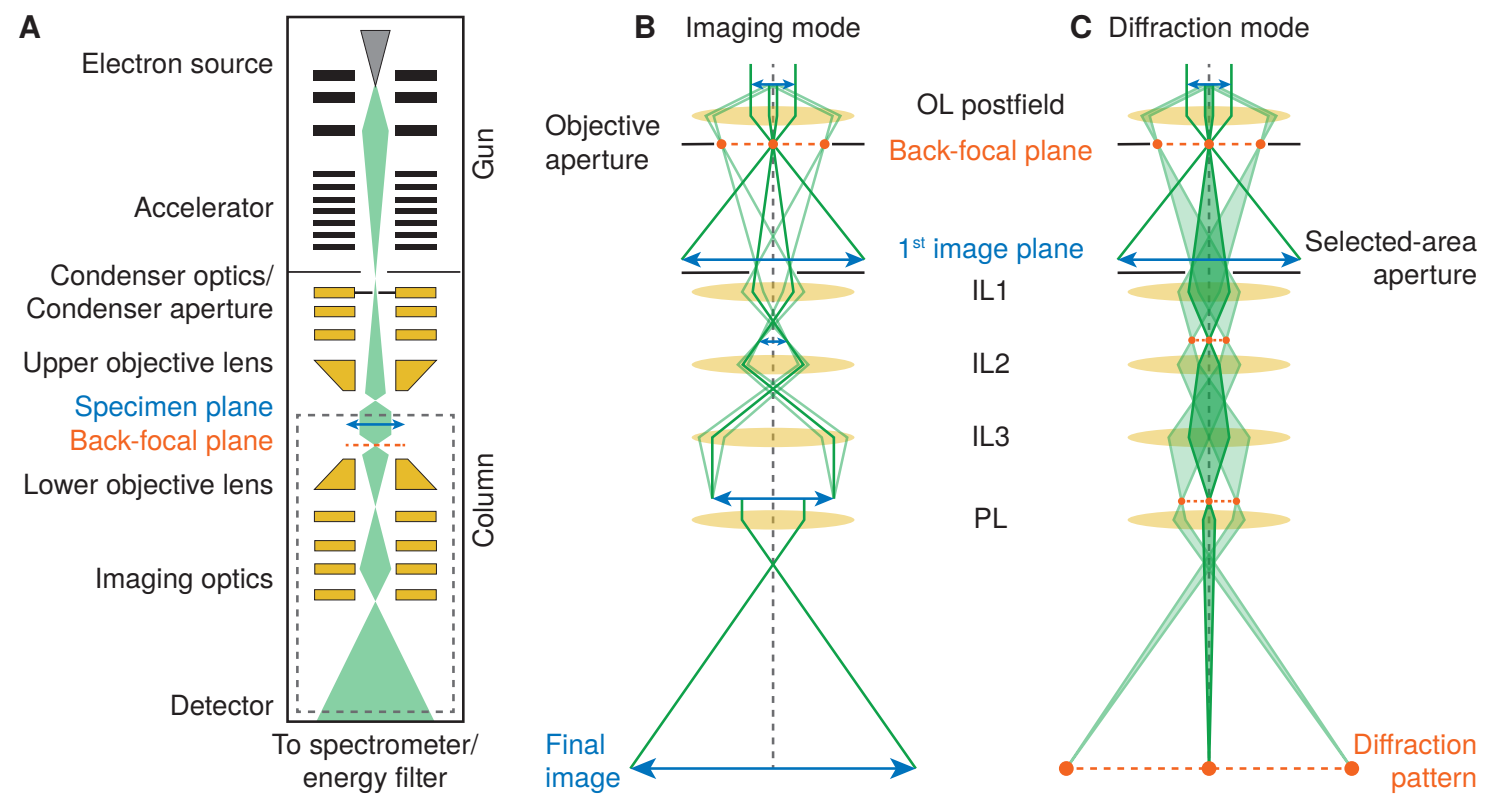

Figure 2.2: Schematic of a TEM and its basic operation modes. (A) Schematic of a TEM. Instead of the detector at the end of the column, the electron beam may also be coupled into an additional spectrometer or energy filter. The dashed gray box indicates the field of view of B and C. (B) Ray diagram of the imaging system set to imaging mode. Note that, although shown below the objective lens (OL) postfield, in reality, the back-focal plane is located within the objective pole piece (see A). (C) Ray diagram of the imaging system set to diffraction mode. 
specimen and the intermediate image plane, the diffraction plane (or back-focal plane, in short BFP) is located. The distance of the BFP from the objective lens is given by the focal length of the lens (typically a few millimeters).

The imaging system, usually consisting of intermediate lenses (IL1 to IL3) and a projector lens (PL), is then responsible for transferring the first intermediate image (or the diffraction pattern in the BFP) onto the electron-sensitive detector. Image magnification and rotation is adjusted by setting appropriate lens currents. Due to this versatility of the optics design, TEMs cover an enormous range of magnifications from $\sim 50 \mathrm{x}$ to $>1,000,000 \mathrm{x}$ in imaging mode (Fig. 2.2B). At the push of a button, the TEM switches to diffraction mode- thus projecting the BFP instead of the first image plane on the detector (Fig. 2.2C).

The focal length of the objective lens is constant in both modes such that the scaling of the BFP and the first intermediate image remain unchanged. This allows for the use of additional circular apertures of variable diameter to shape the electron beam. The objective lens aperture, when introduced into the BFP, filters spatial frequencies contributing to the real-space image in imaging mode. The selected-area aperture is located in the first image plane and allows for the selection of a circular specimen region from which the diffraction pattern is formed.

Specimens for TEM are typically thin films with a thickness, depending on material, electron energy and imaging conditions, between a monolayer of material (92) and roughly $200 \mathrm{~nm}$. TEM specimen holders usually accommodate a circular specimen carrier with a diameter of $3 \mathrm{~mm}$ and are inserted into the side of the column through a vacuum lock. All types of specimen holders can be rotated around the holder axis, while more complex designs have double-tilt, in-plane rotation or heating/cooling functionality $(88)$.

\subsection{Image contrast}

There are two main contrast mechanisms in classical TEM images: Amplitude contrast and phase contrast. Although both contribute to any image, imaging conditions are typically selected such that one type of contrast is dominating (88). For a quantitative description of phase contrast, one has to consider the Abbe theory of image formation. Assuming specimen illumination by a spatially homogeneous plane wave, specimen-specific information is encoded in the real-valued amplitude $a(\vec{r})$ and phase $\varphi(\vec{r})$ of the object wave $\psi(\vec{r})=a \exp (i \varphi)$. The objective lens (and additional, weaker lenses) transfer the object wave function onto the detector, which is only sensitive to the square of the amplitude. Accordingly, the full phase information is lost in an image acquired under in-focus conditions with perfect (aberration-free) lenses (88). 
A

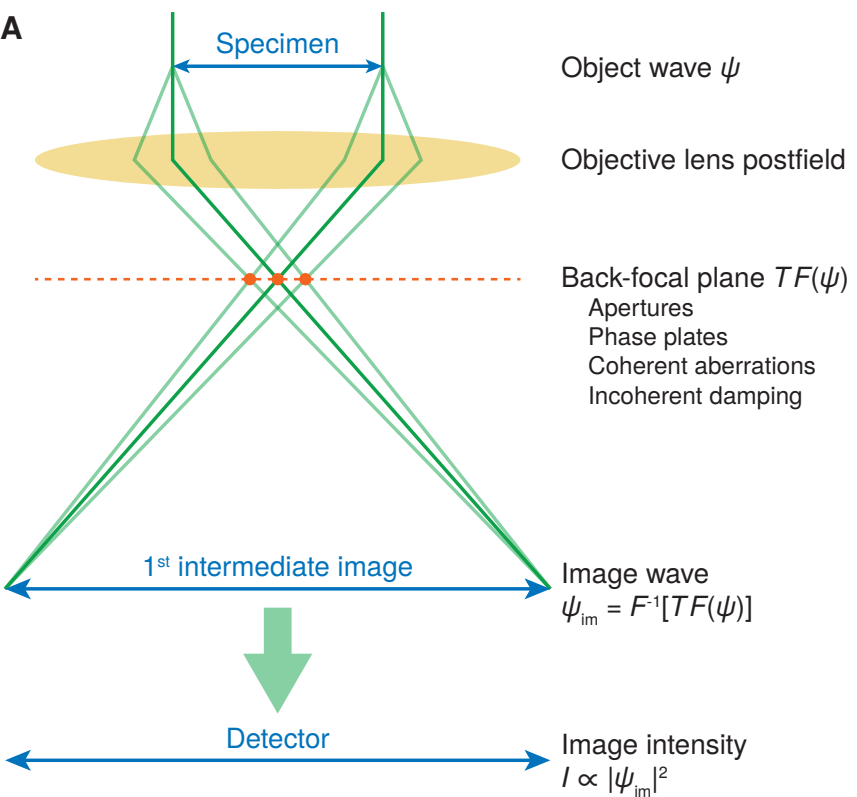

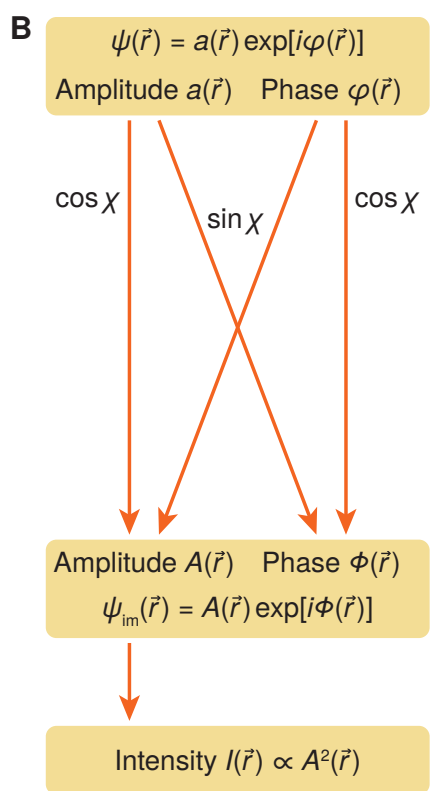

Figure 2.3: Image formation and contrast transfer channels in a TEM. (A) Ray diagram the objective lens region and corresponding expressions for the local electron wave functions along the beam path. The CTF $T(\vec{q})$ encodes all coherent and incoherent modulations that the electron beam undergoes in the imaging process as a function of the scattering vector $\vec{q}$. (B) Contrast transfer channels as controlled by the coherent phase modulations $\chi(\vec{q})$ entering $T(\vec{q})$. Adapted from Ref. (96).

First efforts to obtain phase contrast in an optical microscope were made by FrITs ZeRNIKE in the early 1930s, motivated by the low amplitude contrast of biological specimens $(93,94)$. By introducing a phase plate in the BFP of the microscope that shifted the phase between the unscattered illumination (with a scattering vector ${ }^{2}|\vec{q}|=0$ ) and scattered beams (with $|\vec{q}| \neq 0$ ) by $\chi_{\mathrm{PP}}= \pm \frac{\pi}{2}$, sensitivity to the more pronounced spatial refractive index variations is obtained due to interference between scattered and unscattered beams. In TEMs, Zernike-type phase plates equally lead to a drastic increase in contrast of biological specimens (95).

The effect of an arbitrary phase shift $\chi(\vec{q})$ can be formulated in terms of the contrast transfer function $(\mathrm{CTF}) T(\vec{q}) \propto \exp (-i \chi)$ (Fig. 2.3A). In the BFP, the wave is described by its Fourier transform $\mathscr{F}(\psi)$. The image wave $\psi_{\text {im }}=\mathscr{F}^{-1}[T \mathscr{F}(\psi)]$ in the image plane of the objective lens is obtained by applying the CTF and back-transforming the modulated wave, yielding a modified amplitude $A(\vec{r})$ and phase $\Phi(\vec{r})$. The image intensity on the detector is given by $I(\vec{r}) \propto\left|\psi_{\mathrm{im}}\right|^{2}=A^{2}(88)$.

The complex relationship between amplitude and phase of object and image wave can be

\footnotetext{
${ }^{2}$ Throughout this work, and in accordance with most TEM literature, we adapt the crystallographic definition for the scattering vector and related quantities with $|\vec{q}|=d^{-1}$, where $d$ is the distance between adjacent lattice planes diffracting an electron beam to a scattering vector $\vec{q}$.
} 
derived assuming a weak object imprinting only very small amplitude and phase modulations on the incident wave. In a linear approximation, the transfer of object amplitude and phase to image amplitude and phase is controlled by $\cos \chi$, while a mixing of object amplitude into the image phase and vice versa is proportional to $\sin \chi$ (Fig. 2.3B). From this, the specific effect of a Zernike phase plate becomes obvious. Since $\cos \chi_{\mathrm{PP}}=0$ and $\sin \chi_{\mathrm{PP}}= \pm 1$, only object phase information contributes to the image amplitude of a Zernike phase contrast image (96).

The effect of lens aberrations on the electron wave can also be described by means of a $\vec{q}$-dependent phase plate. This coherent aberration function $\chi_{\mathrm{A}}(\vec{q})$ includes contributions from defocus $\Delta f$, spherical aberration $C_{s}$ and other aberrations. The defocus is given by the distance between the specimen plane and the object plane of the objective lens. The phase contrast in images can be controlled by optimizing the width of the first passband of $\sin \chi_{\mathrm{A}}$, typically by changing $\Delta f$. This is, for example, the basis for high-resolution imaging and the Fresnel mode of Lorentz microscopy, which visualizes the Aharonov-Bohm phase shift acquired by an electron passing through a magnetic field (97).

An objective aperture is typically used to exclude spatial frequencies beyond the first passband of $\sin \chi_{\mathrm{A}}$. This is taken into account in the CTF as an additional aperture function $A(\vec{q})$. Additional envelope terms $E_{i}(\vec{q})$ lead to a damping of the CTF towards large values of $|\vec{q}|$. These include, mainly, the temporal coherence envelope (governed by chromatic aberration, the energy spread of the electron beam and instabilities in the high tension and lens currents) and the spatial coherence envelope (due to limited source coherence). Other envelopes consider the loss of information due to directional specimen drift, specimen vibration and the finite detector size (97). With the aperture function and an effective damping envelope $E(\vec{q})=\prod_{i} E_{i}$, the complete CTF is given by

$$
T(\vec{q})=A(\vec{q}) E(\vec{q}) \exp [-i \chi(\vec{q})]
$$

where $\chi(\vec{q})$ is the sum of all contributions to the $\vec{q}$-dependent phase shift, including defocus, lens aberrations and additional phase plates.

Pure amplitude contrast images are achieved by inserting a smaller objective aperture into the beam, selecting only the direct beam (bright-field imaging, in short BF imaging) or any coherently or incoherently scattered electrons (dark-field or DF imaging). This excludes interference effects between multiple beams and leads to other mechanisms dominating the image contrast.

Spatial intensity variations can indicate mass-thickness contrast due to varying specimen thickness (a thicker specimen scatters more efficiently) or varying specimen composition (incoherent elastic Rutherford scattering scales with the square of the atomic number $Z^{2}$ ). Mass-thickness 
contrast is most useful in non-crystalline or biological specimens. Diffraction contrast due to coherent elastic Bragg scattering appears when selecting a Bragg reflection in the BFP. This can be used to map the presence or absence of specific lattice orientations in the specimen. When using BF imaging on a crystalline specimen, both types of amplitude contrast typically contribute to the image (88).

\subsection{Elastic scattering}

Elastic scattering of electrons in a periodic crystal leads to the formation of Bragg diffraction patterns with discrete spots in the BFP of the TEM. As outlined in the previous section, the wave in the BFP is given by the Fourier transform of the real-space periodicities:

$$
F(\vec{q})=\int_{V} n(\vec{r}) \exp (2 \pi i \vec{q} \cdot \vec{r}) \mathrm{d}^{3} \vec{r}
$$

$F(\vec{q})$ is called the structure factor of a Bragg reflection with scattering vector $\vec{q}$. $V$ is the volume of the crystal unit cell and the real-valued quantity $n(\vec{r})$ is the scattering power. Electrons passing through the crystal are subject to Coulomb interactions with both, the positively charged nuclei and the negatively charged shells of the atoms. X-rays would see the same crystal periodicity and lead to Bragg reflections at the same scattering vectors $\vec{q}$, but experience a different scattering power because electromagnetic radiation is only scattered by the shell electrons (88).

With the substitution $\vec{r}=\vec{r}_{i}+\overrightarrow{\widetilde{r}}$, we can modify the expression for the structure factor to include a sum over all atoms $i$ in the crystal basis with basis vector $\vec{r}_{i}$, while $\overrightarrow{\vec{r}}$ is used to integrate over the charge distribution of every atom individually:

$$
F(\vec{q})=\sum_{i} \exp \left(2 \pi i \vec{q} \cdot \vec{r}_{i}\right) \underbrace{\int_{V_{\text {atom }}} n_{i}(\overrightarrow{\tilde{r}}) \exp (2 \pi i \vec{q} \cdot \overrightarrow{\tilde{r}}) \mathrm{d}^{3} \overrightarrow{\tilde{r}}}_{\text {Atomic scattering factor } f_{i}(\vec{q})}
$$

Accordingly, the structure factor is given by a sum over the atomic scattering factors $f_{i}(\vec{q})$ of all atoms in the crystal unit cell weighted by a phase factor. Atomic scattering factors are typically given as a function of $\sin \theta / \lambda$, where $\theta$ is the scattering semi-angle and the electron wavelength $\lambda$ is given in units of $\AA$ (98). Via Bragg's law, we find

$$
\frac{\sin \theta}{\lambda}=\frac{1}{2 d}=\frac{|\vec{q}|}{2}
$$


Parametrized as a sum of $n$ Gaussians of the form

$$
f\left(\frac{\sin \theta}{\lambda}\right)=\sum_{i=1}^{n} a_{i} \exp \left[-b_{i}\left(\frac{\sin \theta}{\lambda}\right)^{2}\right],
$$

atomic scattering factors for electron diffraction can be calculated from values for $a_{i}$ and $b_{i}$ as tabulated, e.g., in Refs. $(98,99)$.

The positions of basis atoms $\vec{r}_{i}$ are typically given in fractional coordinates $\left(u_{i}, v_{i}, w_{i}\right)$ in a (possibly non-Cartesian) coordinate system that is formed by the real-space lattice vectors $\vec{a}_{j}$ (such that $\vec{r}_{i}=u_{i} \vec{a}_{1}+v_{i} \vec{a}_{2}+w_{i} \vec{a}_{3}$ ). The corresponding reciprocal lattice vectors $\vec{b}_{k}$ are defined by $\vec{a}_{j} \cdot \vec{b}_{k}=\delta_{j k}$ with the Kronecker delta function $(j, k \in[1,3]$ for a three-dimensional crystal). Only specific reciprocal lattice vectors $\vec{q}_{h k l}=h \vec{b}_{1}+k \vec{b}_{2}+l \vec{b}_{3}$ lead to constructive interference of the phase factors in all scattering unit cells, and thus a non-zero intensity $I_{h k l} \propto\left|F_{h k l}\right|^{2}$ in the diffraction pattern:

$$
F_{h k l}=\sum_{i} f_{i}\left(\vec{q}_{h k l}\right) \exp \left[2 \pi i\left(u_{i} h+v_{i} k+w_{i} l\right)\right]
$$

If $\vec{q}$ is no reciprocal lattice vector, the sum of the phase factors over all scattering unit cells adds up to zero-no elastically scattered intensity can be detected at this scattering vector.

The Miller indices $(h k l)$ denote not only a specific Bragg reflection, but also a family of real-space lattice planes orthogonal to $\vec{q}_{h k l}$ with an inter-plane distance $d_{h k l}=1 /\left|\vec{q}_{h k l}\right|$. This way of calculating diffracted intensities is called the kinematic approximation and assumes a sufficiently thin specimen such that multiple scattering events play no dominant role (88).

\subsection{Specimen preparation}

A wide range of preparation methods for TEM specimens are available, depending on the experimental requirements and material availability. These reach from the investigation of ground and powder materials on a support film to complex multi-step preparation procedures starting from suitable bulk material or thin films grown on a substrate. Until the advent of focused ion beam (FIB) devices, the standard technique in materials science was a combination of mechanical preparation and successive broad-beam milling using argon ions. However, mechanical polishing procedures to thin certain materials down to an atomically thin wedge are also available (88). Today, focused gallium or helium ions are used in FIB devices to prepare thin films of material, so-called lamellae $(100,101)$. 
Some materials can be grown directly on electron-transparent membranes and can even be structured using electron-beam lithography (102-104). Commercially available TEM membrane types include amorphous silicon nitride membranes with millimeter-scale lateral sizes and thicknesses down to $5 \mathrm{~nm}$. These membranes have low internal stress, are smooth and chemically inert. For biological samples in cryo-TEM techniques, equally thin carbon films supported by copper or gold grids are a popular choice (88). Another route to TEM specimens for the case of layered materials with weak interlayer bonding is the exfoliation or "scotch-tape" technique (105).

Ultramicrotomy is a related preparation technique originating from the biological sciences. An ultramicrotome is a mechanical device that allows for cutting specimens into thin slices called sections. For this purpose, a specimen block is mounted on an arm, that, in operation, moves downwards and slides the block along an atomically sharp knife edge (Fig. 2.4A). The sections then float in a water trough until transferred onto a suitable specimen carrier (88). In contrast to most other TEM preparation methods, ultramicrotomy sections can reach lateral sizes of several hundreds of micrometers with very homogeneous thickness distributions (Fig. 2.5).

In the preparation of material science specimens, ultramicrotomy also has its advantages as demonstrated in Chapters 4 and 5. In this case, the typical fixation and dehydration procedures required for biological specimens are not necessary. Special resin and a mold are used to embed the material in a specimen block. The hardness of the resin should be as close as possible to that of the embedded material. If the specimen material is rigid enough, which holds true for
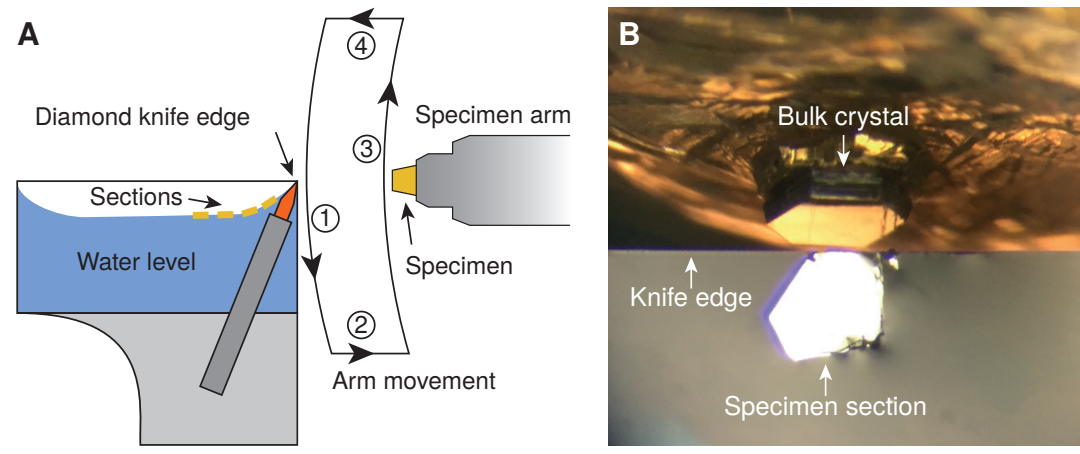

Figure 2.4: Specimen preparation using ultramicrotomy. (A) Schematic of the preparation process and the movement of the specimen arm. In operation, the arm moves downwards (1) and slides the specimen block along the atomically sharp edge of a diamond knife, resulting in an ultrathin section of the material. After cutting, the arm retracts slightly (2), moves upwards (3), feeds forward by the same length plus the desired section thickness (4), and cuts the next section (1). Ultrathin sections float on the water surface until transferred onto a specimen carrier. (B) Photograph of the diamond knife edge with a fresh section of graphite floating on the water surface taken through the binoculars of the ultramicrotome. The hexagonal bulk crystal is mounted on a block of gold and shown in the retracted position (3). 

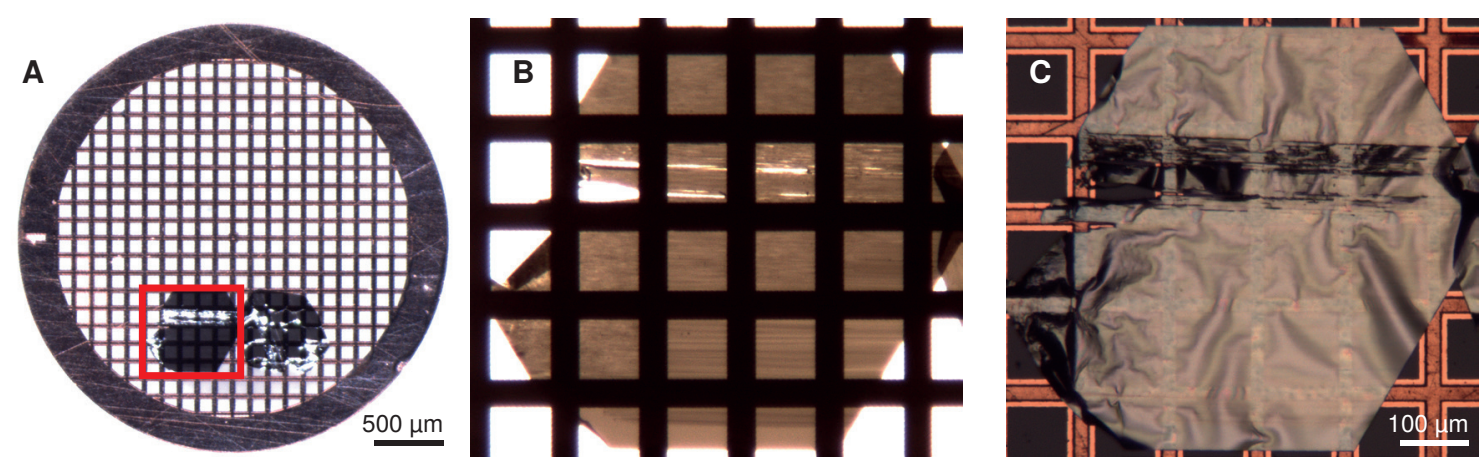

Figure 2.5: Light microscopy images of ultramicrotomed graphite films on a grid. (A) Two graphite films with $30 \mathrm{~nm}$ nominal thickness on a 200 lines per inch copper grid. (B) One of the graphite films observed in transmitted-light mode. The horizontal tear is due to a crystal defect of the bulk crystal. The field of view is indicated by the red box in A. See C for the scale bar. (C) The same graphite film as in B observed in reflected-light mode.

most (and even layered) crystalline materials, embedding can be completely abandoned and the material can be directly mounted on the specimen arm of the ultramicrotome as shown in Fig. 2.4B (106).

Before sectioning, the cured specimen block must be trimmed in order to expose a small facet to the knife edge. Often, razor blades and glass knives are used for this purpose. After trimming, the exposed facet is sectioned in the ultramicrotome. Special ultramicrotomy diamond knives allow for section thicknesses even below $50 \mathrm{~nm}$. For very thin bulk crystals, i.e., with a thickness of only a few tens to hundreds of microns, it may be worthwhile to glue the specimen to a block of soft material such as gold (Fig. 2.4B). In case the complete crystal has been sectioned, the diamond knife will cut into soft gold instead of the stainless-steel specimen holder which prevents damage to the knife edge (106).

When floating in the water trough, individual sections can be carefully moved and manipulated using an eyelash hair. There are many different methods to retrieve the sections and place them on a support film or copper grid. One method is to use the eyelash to guide the section towards a TEM grid held slightly tilted under water. When gently rising the grid above the water level, the sections remain on the grid (106).

However, there are also other tools commercially available, such as the "perfect loop". This tool essentially consists of a handle with a $3 \mathrm{~mm}$ ring attached. The ring can be used to lift a drop of water from the trough using the surface tension of the water, and with it the section that was floating on the water surface. When lowering the perfect loop onto a TEM grid positioned on a piece of filter paper, the water is absorbed by the paper, thus fixing the dried section on the grid. 


\subsection{Specimen thickness measurements}

There are several techniques that can help to assess the thickness of TEM specimens in situ and to a varying degree of accuracy. Analyzing convergent-beam diffraction patterns, for example, can give quite precise results for crystalline specimens (107). However, the most easily accessible technique relies on using EELS or energy-filtered transmission electron microscopy (EFTEM). EELS spectra show the probability of a specific energy loss due to inelastic scattering. Assuming independent events, the probability $P_{n}$ of the occurrence of $n$ of those scattering events while passing through the specimen is given by Poisson statistics (77):

$$
P_{n}=\frac{I_{n}}{I_{t}}=\frac{\left(\frac{t}{\lambda}\right)^{n}}{n !} \exp \left(-\frac{t}{\lambda}\right)
$$

Here, $I_{t}$ is the total incoming beam intensity, $I_{n}$ is the intensity of $n$-fold inelastically scattered electrons, $t$ is the absolute specimen thickness, and $\lambda$ the effective mean free path (MFP) in the material under the specific experimental conditions. Specifically, these include the initial electron energy and the beam convergence and collection angles. The most easily accessible quantity along with $I_{t}$ is $I_{0}$, the intensity of the zero-loss peak (ZLP), i.e., the intensity of unscattered electrons in the beam. Setting $n=0$ yields the formula for the EELS log-ratio technique for specimen thickness measurement:

$$
\frac{t}{\lambda}=\log \left(\frac{I_{t}}{I_{0}}\right) .
$$

This approach has been employed in sections 4.3 and 5.2.3, and allows for calculating the specimen thickness in units of the MFP from the integrated area under the ZLP and the area under the full spectrum. For this purpose, a suitable cutoff between ZLP and the low-loss part of the spectrum must be chosen (assuming that both ZLP and low-loss tails beyond the cutoff cancel each other), or the ZLP must be fitted using a model function. While the former is the typical approach for recording thickness maps using EFTEM, the latter is typically used for extracting the specimen thickness from EELS spectra.

Care must be taken to choose the dispersion of the spectrometer such that only a negligible amount of intensity lies outside of the recorded energy range. Sometimes, this approach is already sufficient for a quick specimen thickness estimate, as $t / \lambda<1$ is desirable for most TEM experiments. In order to obtain a value for $t$, one has to obtain an estimate for the MFP $\lambda$. Alternatively, the absolute thickness can also be calculated directly from the shape of the EELS spectrum (or, more specifically, the deconvoluted single-scattering spectrum) by Kramers-Kronig analysis, but this requires knowledge of the specimen's complex dielectric function (108). 
MaLis et al. have parametrized the MFP as a function of the collection semi-angle $\beta$ in mrad, the beam energy $E_{0}$ in $\mathrm{keV}$, and a relativistic factor $F$ for use in materials where the refractive index is much larger than unity (109):

$$
\lambda(\mathrm{nm})=\frac{106 F E_{0} / E_{\mathrm{m}}}{\log \left(2 \beta E_{0} / E_{\mathrm{m}}\right)} .
$$

The relativistic factor takes the form

$$
F=\frac{1+E_{0} / 1022}{\left(1+E_{0} / 511\right)^{2}}
$$

and the average energy loss $E_{\mathrm{m}}$ includes the element dependency. While tabulated values are available for some elements (77), $E_{\mathrm{m}}$ can be approximated by $E_{\mathrm{m}}=7.6 Z^{0.36}$ as a function of the atomic number $Z$. For compounds, either a simple average $\left(\bar{Z}=\sum_{i} f_{i} Z_{i}\right)$ or an effective atomic number

$$
Z_{\mathrm{eff}}=\frac{\sum_{i} f_{i} Z_{i}^{1.3}}{\sum_{i} f_{i} Z_{i}^{0.3}}
$$

derived from the Lenz scattering model (110) replaces $Z$, where $Z_{i}$ is the atomic number of each atom species occurring in the compound with atomic fraction $f_{i}$. This leads to a prediction of $\lambda$ within $20 \%$ accuracy (109).

A more recent approach by Iакоuвоvsкi $e t$ al. models $\lambda$ as a function of specimen density $\rho$ in $\mathrm{g} / \mathrm{cm}^{3}$ instead of the atomic number, resulting in the formula

$$
\lambda(\mathrm{nm})=\frac{200 F E_{0}}{11 \rho^{0.3}} / \log \left(\frac{\alpha^{2}+\beta^{2}+2 \theta_{\mathrm{E}}^{2}+\left|\alpha^{2}-\beta^{2}\right|}{\alpha^{2}+\beta^{2}+2 \theta_{\mathrm{C}}^{2}+\left|\alpha^{2}-\beta^{2}\right|} \cdot \frac{\theta_{\mathrm{C}}^{2}}{\theta_{\mathrm{E}}^{2}}\right)
$$

with the same relativistic factor $F, \theta_{\mathrm{C}}=20 \mathrm{mrad}$ and $\theta_{\mathrm{E}}=5.5 \rho^{0.3} / F E_{0}$. Additionally, the convergence semi-angle $\alpha$ (in mrad) is taken into account (111). This yields an improvement over the formula by MaLis et al. in most cases (77).

\subsection{Electron-sensitive detectors}

Even most modern TEMs are still equipped with a cathodoluminescent screen that allows for live observation of the electron beam with the bare eye. Before the advent of digital electron cameras, TEM images and diffraction patterns were recorded on photographic film and chemically developed to obtain the final micrograph. Nowadays, virtually any TEM in operation is equipped 
with a camera recording two-dimensional, pixelated image data. First TEM cameras were based on charge-coupled devices (CCDs) that are not radiation hard. Therefore, incoming electrons are typically converted to photons in a scintillator and transferred to the CCD sensor through a fiber-optic plate, where electric charges are collected in individual pixels. After exposure, every pixel row on the detector is shifted towards the detector edge for read-out. Here, the same analog-to-digital converter sequentially converts the collected charges per pixel into a digital number that is proportional to the collected beam intensity $(112,113)$.

More current camera designs use active pixel sensors that have separate read-out electronics for every individual pixel. These are based on CMOS (complementary metal-oxide semiconductor) technology and therefore often called CMOS sensors. By eliminating the necessity of shifting pixel rows through the detector, these designs allow for much faster frame rates and reduce undesired effects occurring in CCDs, such as smearing (due to continued exposure during read-out) and blooming (due to overexposed pixels) $(114,115)$.

However, the use of scintillators to convert electron events to photons reduces the detective quantum efficiency (DQE), which describes the loss of signal-to-noise ratio (SNR) in the detection process as a function of spatial frequency (113). This led to the development of direct electron detectors which can be directly exposed to the electron beam. Hybrid pixel detectors, on the one hand, are direct electron detectors consisting of a sensor layer bump-bonded to a separate chip with the CMOS read-out electronics. These event-driven detectors count every individual incoming electron and either generate a classical image or a continuous data stream of electron events. While representing the state of the art for particle physics and synchrotron experiments, in electron microscopy they suffer from the disadvantage that typical pixel sizes are large in comparison to CCD and CMOS cameras $(113,116,117)$.

Monolithic active pixel sensors, on the other hand, are direct electron detectors based on back-thinned, radiation-hardened CMOS chips (118). This design allows for much smaller pixel sizes than is currently possible with hybrid pixel technology. Additionally, fast read-out with frame rates up to hundreds of frames per second can be realized. This allows for operating these cameras in two different modes: In integrating mode, the behavior is very similar to that of scintillator-based CCD and CMOS cameras. Every pixel accumulates charges during exposure until the full frame is read out at once. In counting mode, one makes use of the fast read-out rates of the chip design. If the electron dose is low enough, i.e., on the order of one electron per 100 pixels or even lower, individual electron events can be identified due to the high DQE and SNR. The electron counting may either be done on-chip or on the camera computer. The result is then obtained by adding up tens, hundreds or even thousands of individual low-dose 
frames. Due to the event localization in the counting process, the attainable dynamic range is much higher than with conventional detectors, and Poisson noise (or shot noise) is the main source of noise in the final image $(113,119)$.

\subsection{Ultrafast transmission electron microscopy}

Temporal resolution in conventional TEMs as described in the previous sections is achieved by using a detector that can be read out fast enough to sample the time evolution of a specimen undergoing reversible or irreversible dynamics in situ. While conventional full-frame cameras restrict the resolution to the millisecond or sometimes microsecond time scale, some event-driven detectors can time-stamp individual electron events with nanosecond precision (116). Elementary excitations of electrons, lattice and spins, however, evolve on femto- to picosecond time scales. In order to reach the time resolution required for imaging this kind of dynamics, ultrafast TEMs typically use pulsed electron sources and specimen excitation in a laser pump/electron probe scheme. First, an ultrashort laser pulse (pump) is used to drive the specimen out of its equilibrium state. Subsequently, one or more ultrashort electron pulses (probe) are used to image the transient state of the specimen after a temporal delay $\Delta t(120,121)$.

In more detail, two different approaches to time-resolved TEM have emerged. From the 1980s on, Bostanjoglo et al. developed the first time-resolved TEM with $30 \mathrm{~ns}$ temporal resolution $(122,123)$. It was able to record three subsequent images of irreversible dynamics using three individual photoemitted electron pulses after a single impulsive laser excitation of the specimen (124). An electrostatic beam deflector was used to record the image series on a single detector frame, thus circumventing the need for a fast detector. This single-shot approach was further developed at Lawrence Livermore National Laboratory (LLNL) and termed dynamic transmission electron microscopy (DTEM) $(125,126)$. In its latest reported state, the DTEM system records an array of up to $16 \times 16$ sub-images on the detector with down to $10 \mathrm{~nm}$ spatial resolution using $10^{8}$ to $10^{9}$ electrons in $15 \mathrm{~ns}$ pulses (127-129). Combined with a compressive sensing approach, even more sub-images can be encoded in the same detector frame (129).

In contrast, ultrafast transmission electron microscopy (UTEM) uses a stroboscopic pump/ probe scheme to record movies of reversible dynamics. The image at every single temporal delay $\Delta t$ is integrated over millions of consecutive optical pump and electron probe pulses. In contrast to the DTEM approach, UTEMs are mostly operated in a single-electron regime, in which Coulomb repulsion within the individual electron pulses can be neglected. This allows 
for the realization of UTEMs with femtosecond electron pulse durations and a spatial resolution comparable to that of conventional TEMs (120).

The first stroboscopic UTEM was presented by the group of Ahmed ZewaIL at the California Institute of Technology $(\text { Caltech })^{3}(83,130,131)$. Since then, various implementations of the UTEM methodology have been put into operation in laboratories around the world (66, 132-146). Despite the fundamentally identical working principle, a decisive influence on the electron pulse properties is found in the specific design of the electron source. The most common implementation employs a flat photocathode to emit a single ultrashort electron pulse for each ultrashort laser pulse absorbed by the emitter. A typical choice, due to a low work function of only $2.4 \mathrm{eV}$, is a lanthanum hexaboride $\left(\mathrm{LaB}_{6}\right)$ emitter in a truncated-cone shape $(120)$. These emitters are often used in conventional TEMs where they are operated in a thermionic regime. A continuous electron beam is emitted from the $\mathrm{LaB}_{6}$ surface when heated by an electrical current flowing through the filament holding the emitter. In ultrafast operation, the work function of the cold emitter is overcome by the photon energy of the photoemission laser. Besides the original implementation at Caltech, $\mathrm{LaB}_{6}$-based UTEMs are operated, for example, at EPFL in Lausanne (133), at the University of Minnesota (136) and at KTH in Stockholm (138).

However, with typical diameters of tens of micrometers, $\mathrm{LaB}_{6}$ emitters emit a relatively incoherent electron beam due to the large effective source size. Applications demanding a high source coherence, such as electron holography, coherent nanodiffraction or quantum optics experiments are therefore challenging in thermionic UTEMs $(120,147,148)$. In conventional TEMs, beam coherence could be increased by spreading the beam in the gun or the illumination system. In the case of UTEM, however, the associated loss in beam current is often not tolerable, which led to the development of high-coherence electron sources based on metallic nanotips $(149,150)$, for example, at the University of Göttingen (66), at the University of Toulouse (140) and at the Chinese Academy of Sciences (143).

The Göttingen UTEM is based on a JEOL JEM-2100F Schottky field emission microscope (66) and was the first to use a tip-shaped photocathode in time-resolved operation (Fig. 2.1A to C). The Schottky field emitter shares the advantage of thermionic emitters that it can either be operated in a continuous mode at a temperature of $1800 \mathrm{~K}$ and extraction fields on the order of $0.5 \mathrm{~V} / \mathrm{nm}$ to $1 \mathrm{~V} / \mathrm{nm}$ at the tip apex, or in photoemission mode. When operating the emitter below $1400 \mathrm{~K}$, continuous electron emission is fully suppressed and photoelectron pulses are generated by the photoemission laser focused onto the tip apex. The emission process is supported by a zirconium oxide overlayer, reducing the work function to about $2.9 \mathrm{eV}$ at the $(100)$ front facet of

\footnotetext{
${ }^{3}$ The ZEwAIL group used the abbreviation UEM (for ultrafast electron microscopy) instead of UTEM.
} 

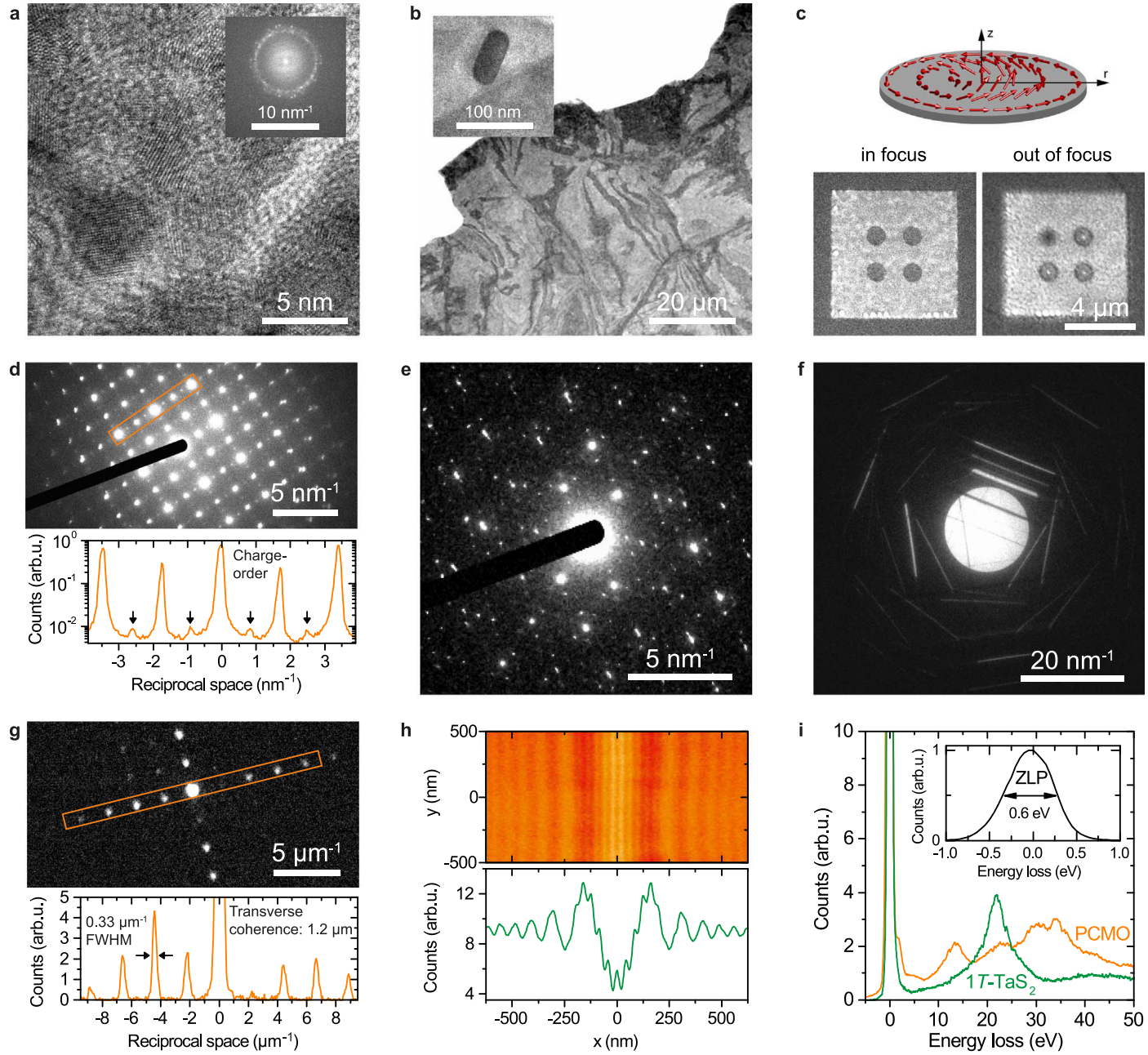

Figure 2.6: Exemplary experimental results achievable with the current status of the Göttingen UTEM instrument. (A) High-resolution TEM (HRTEM) micrograph of Au/Pd particles on an amorphous carbon film. Visible lattice planes with 2-Å spacing demonstrate the resolution capabilities of the modified instrument (here: using thermal electron emission). Inset: Fourier transform of a four times larger sample region. (B to I) Measurements acquired with photoelectron beams (typical acquisition times 5-60 s) and at an electron energy of $120 \mathrm{keV}$. (B) Bright-field image of an ultra-microtomed $50 \mathrm{~nm}$ thin sample of $1 \mathrm{~T}-\mathrm{TaS}_{2}$ showing bending contrast of the thin-film membrane. Close-up: drop-casted gold nanorod on the sample surface. (C) Lorentz imaging provides magnetic contrast in UTEM as demonstrated for permalloy islands on a silicon nitride support (see also Ref. (151)). The out-of-focus image reveals the existence of a magnetic vortex in each of the four islands (visible as black and white features, respectively, depending on vortex orientation). The magnetic structure of a single vortex is schematically depicted in the upper panel. (D) Diffraction pattern of the charge-ordered phase of an ion-polished PCMO $\left(\mathrm{Pr}_{0.7} \mathrm{Ca}_{0.3} \mathrm{MnO}_{3}\right)$ plan view sample. Weak superstructure spots are visible halfway between the lattice reflections. (E) Diffraction pattern of the nearly commensurate charge density wave (NC-CDW) phase of $1 \mathrm{~T}-\mathrm{TaS}_{2}$. The first-order NC-CDW diffraction spots are hexagonally arranged around structural reflections. (F) Convergent beam electron diffraction (CBED) pattern of an exfoliated $100 \mathrm{~nm}$ thick single-crystalline graphite flake. (G) High dispersion diffraction pattern of a $463 \mathrm{~nm}$ spaced grating replica, demonstrating 1.2- $\mu \mathrm{m}$ transverse coherence lengths. (H) Electron hologram obtained using a Möllenstedt biprism at a filament voltage of $9 \mathrm{~V}$, emphasizing the photoelectron coherence properties achievable in the UTEM. (I) Electron energy loss spectra of $1 \mathrm{~T}-\mathrm{TaS}_{2}$ and PCMO. Inset: zero-loss peak (ZLP) with a FWHM of $0.6 \mathrm{eV}$. Figure and caption reprinted from Ref. (66). 
the tungsten tip. This leads to emission of the pulsed electron beam from the front facet only, and thus greatly enhances the beam coherence in comparison to thermionic emitters (Fig. 2.1D to F). Considering brightness limitations, most imaging, diffraction and spectroscopy applications that are possible with an unmodified microscope can be performed at the Göttingen UTEM as well (Fig. 2.6).

The versatility of the Göttingen UTEM is also reflected by the diverse applications benefiting from the high-coherence pulsed beam (66). An early quantum optics experiment (and later follow-ups) investigated the inelastic interaction of the free electrons with optical near-fields $(147,148,152-154)$. While this kind of electron-photon interaction does not fundamentally require a pulsed electron beam, the necessary optical near-field strengths could initially only be achieved in pulsed schemes (155). Meanwhile, the use of optical resonators for the near-field confinement has opened up perspectives for continuous-beam interactions as well $(156,157)$. By temporally scanning optical and electron pulses across each other to obtain an electron-photon cross-correlation, the effect is routinely used to determine the electron pulse duration as reported in Ref. (66) and used in Chapter 5.

The femtosecond temporal resolution of the Göttingen UTEM has been harnessed by another set of experiments imaging optically driven (102) and current-driven magnetization dynamics $(103,104)$ on the nanoscale. Finally, the observation and analysis of strain waves in a singlecrystalline graphite film has been demonstrated, which contributes to the field of ultrafast structural dynamics (158). This field is of central importance to the present thesis and will be reviewed in the following section.

\subsection{Ultrafast imaging of structural and electronic dynamics}

While the UTEM quantum optics experiments certainly opened up novel experimental perspectives, investigations of electronic, structural and magnetic properties look back at a long tradition of conventional TEM investigations of the same or similar materials. However, by adding femtosecond time resolution, completely new insights into the couplings between the different degrees of freedom are obtained. The purpose of this section is to summarize a number of central experiments investigating ultrafast material dynamics using UTEM.

One advantage of the UTEM approach over related stroboscopic techniques is the availability of a number of contrast methods that allow for access to different degrees of freedom of the specimen in the same experimental setting. In some widely investigated materials, such as vanadium dioxide $\left(\mathrm{VO}_{2}\right)$ and different TMDCs (Chapter 3), electron-lattice coupling and strong 
correlation effects lead to physical phenomena that can only be fully understood by investigating both, the structural and electronic contributions. These phenomena include, for example, metalinsulator phase transitions (MITs) (159) and the formation of charge-density waves (CDWs) (160).

Ultrafast EELS, for example, can be used to gain insights into the electronic component of material dynamics as demonstrated for graphite, both in the low-loss and high-loss regimes (161164). High-loss EELS spectra allow to investigate the dynamics of chemical bonds on ultrafast time scales, while the electronic, magnetic and optical properties of materials are controlled by low-energy excitations $(165,166)$. Both types of observations are rather challenging: The low inelastic scattering cross-section for loss energies $>100 \mathrm{eV}$, on the one hand, leads to long integration times in low-dose ultrafast experiments. On the other hand, the finite width of the ZLP in TEMs without a monochromator covers relevant low-loss features such as the dynamics of band gaps in MITs.

Another way to obtain information about the transient electronic properties of materials exploits the inelastic electron-photon interaction. It has been shown in the past that spatial mapping of energy sidebands allows for imaging of plasmonic fields in metallic nanostructures (photon-induced near-field electron microscopy or PINEM) $(166,167)$. The intensity of the optical near-field depends on the dielectric function of the material-a property that undergoes a pronounced change in a MIT and that can thus serve as a source of image contrast, as exemplified using $\mathrm{VO}_{2}$ nanowires (168).

The structural component of the MIT in $\mathrm{VO}_{2}$ has been investigated in a number of diffraction experiments using UTEM $(131,169)$, dedicated UED setups $(24,64,170-173)$ and x-rays (174, 175). Diffraction experiments have the distinct advantage over all-optical experiments that even minute structural changes, such as the formation of vanadium dimers in the MIT (which is equivalent to the formation of a CDW along the vanadium chains), lead to the emergence or disappearance of distinct diffraction orders corresponding to the changes in crystal symmetry. These are spatially separated from other diffraction orders in the diffraction pattern, such thatgiven reasonably low inelastic scattering background intensity and detectors with sufficiently high DQE - even very weak changes can be observed and analyzed. The intensity of the vanishing diffraction orders can be considered an order parameter of the structural phase transition (24).

While UED experiments can also be conducted using UTEM devices $(28,131,134,163$, 169, 176-178), pioneering works have proven that the strength of UTEM lies in the nanometer spatial resolution it contributes to structural dynamics investigations $(56,57,131,169)$. The stroboscopic UTEM images in Fig. 2.7A demonstrate the change of contrast in a polycrystalline 
A

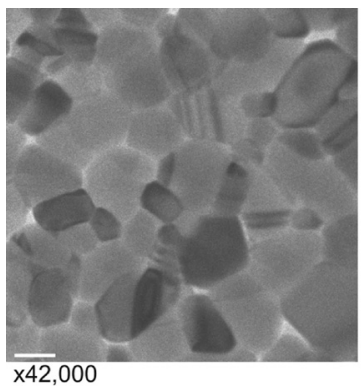

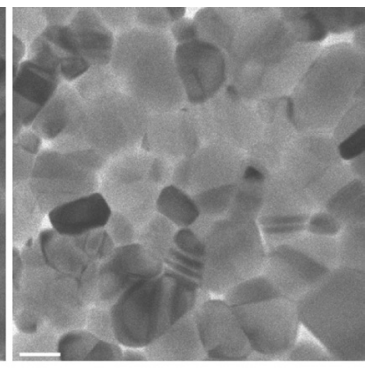

B

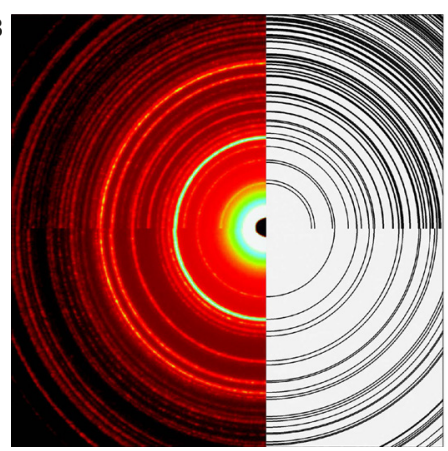

$M$

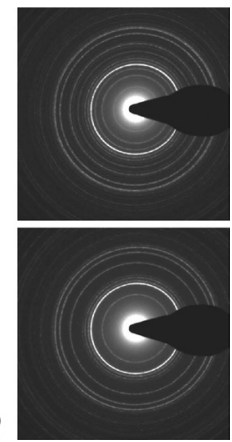

Figure 2.7: Ultrafast TEM images and diffraction patterns of $\mathbf{V O}_{2}$. (A) Images of the polycrystalline specimen in the monoclinic (M) room-temperature phase (left) and in the rutile (R) high-temperature phase (right) (before and after time-zero). The scale bar length is $100 \mathrm{~nm}$. (B) Diffraction patterns corresponding to the $\mathrm{M}$ and $\mathrm{R}$ phases (left) compared to theoretical ring positions (center). Raw diffraction patterns of both phases are shown as well (right). The higher symmetry of the $\mathrm{R}$ phase leads to the disappearance of a number of diffraction orders that are prominent in the dimerized M phase. Reprinted from Ref. (131). Copyright 2006 National Academy of Sciences.

$\mathrm{VO}_{2}$ specimen before and after optical excitation. While the overall shape of the individual crystallites remains unaffected by the phase transition, transient features can be observed within. Due to a strong contribution of diffracted beams that are not sensitive to the structural changes, these features carry rather low contrast. Simultaneously, the changes between the diffraction patterns observed in the monoclinic $(\mathrm{M})$ room-temperature and the rutile $(\mathrm{R})$ high-temperature phase are quite obvious (Fig. 2.7B).

This emphasizes the necessity of contrast enhancement techniques in the real-space imaging of structural dynamics using UTEM, as first demonstrated by the example of an organometallic nanoparticle undergoing a spin-crossover phase transition (58). Structural strain in the particle is released during the transition, causing a change in morphology (Fig. 2.8D). This leads to local changes in diffracted intensities that reflect the new lattice orientation. Using an individual circular aperture, a diffraction spot in the BFP can be selected (red circle in Fig. 2.8C) to obtain DF images of the phase transition dynamics (Fig. 2.8B). In this very specific case, an intense diffraction order with strong sensitivity to the crystal deformation was selected such that the corresponding BF images show a complementary spatial intensity distribution (Fig. 2.8A).

Diffraction contrast as showcased in Figs. 2.7 and 2.8 represents a typical contrast mechanism in real-space UTEM images (56-62). When carefully evaluated, the obtained BF and DF images also allow for quantitative insights into the structural dynamics. This includes the observation of acoustic phonon modes in BF images, as demonstrated using graphite (56) and in a number of recent works on the TMDCs $\mathrm{TaS}_{2}, \mathrm{WSe}_{2}$ and $\mathrm{MoS}_{2}$ (59-61). Using this approach, the propagation of individual phonon wavefronts can be analyzed in space and time (Fig. 2.9). 

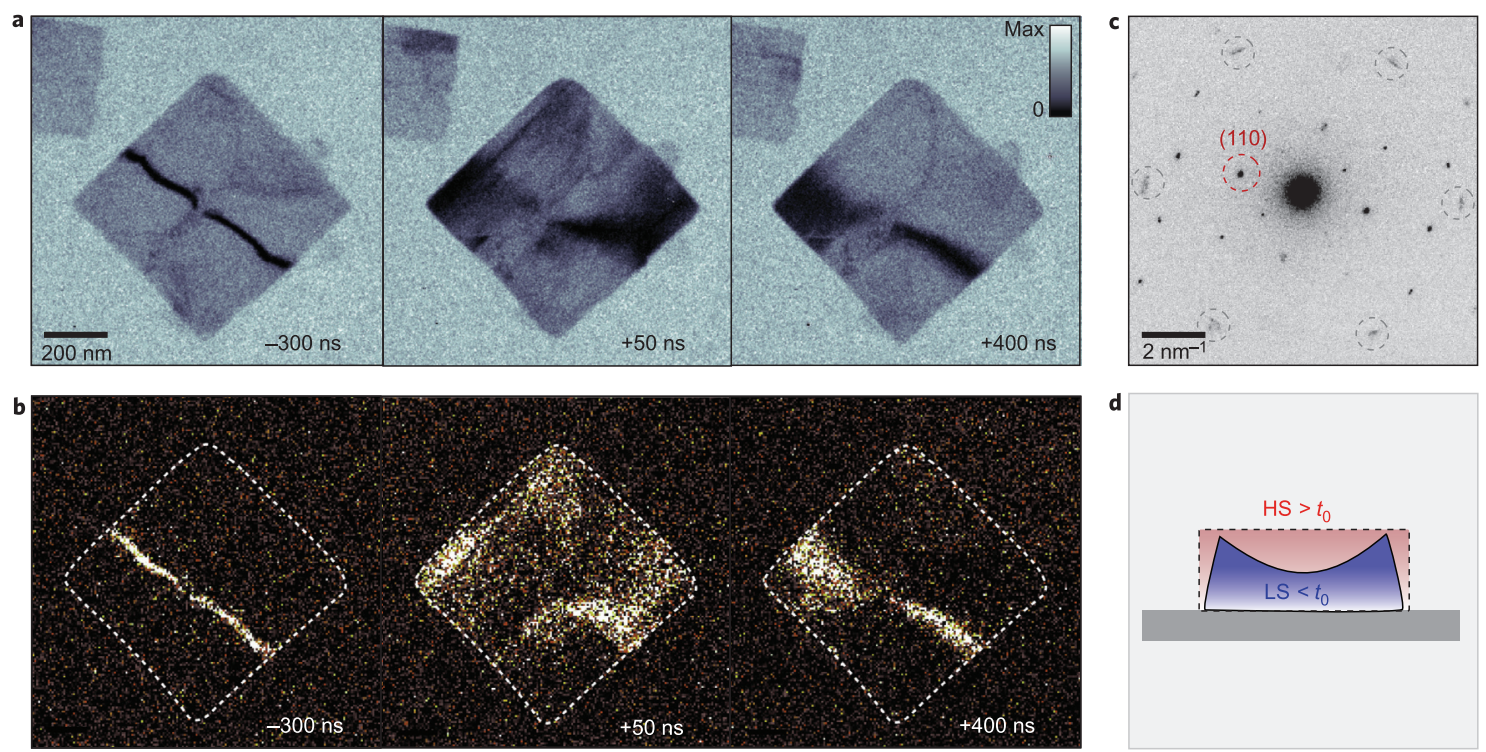

Figure 2.8: Single-nanoparticle morphology dynamics. (A) BF images of a nanoparticle at three different temporal delays. (B) Corresponding DF images obtained by selecting the (1 110$)$ diffraction order using a circular objective aperture in the BFP. $(\mathbf{C})$ Diffraction pattern of the single nanoparticle slightly tilted away from the $\left[\begin{array}{lll}0 & 0 & 1\end{array}\right]$ zone axis. The $\left(\begin{array}{lll}1 & 1 & 0\end{array}\right)$ reflection and diffraction peaks of the graphite substrate are encircled in red and gray, respectively. (D) Side view of the nanoparticle. Before time-zero, the particle is in a strained, diamagnetic low-spin (LS) state. After excitation, the particle transitions into a high-spin (HS) state which releases the strain. Reprinted by permission from Springer Nature Customer Service Centre GmbH: Springer Nature, Nature Chemistry, Ref. (58), Copyright 2013 Nature Publishing Group.
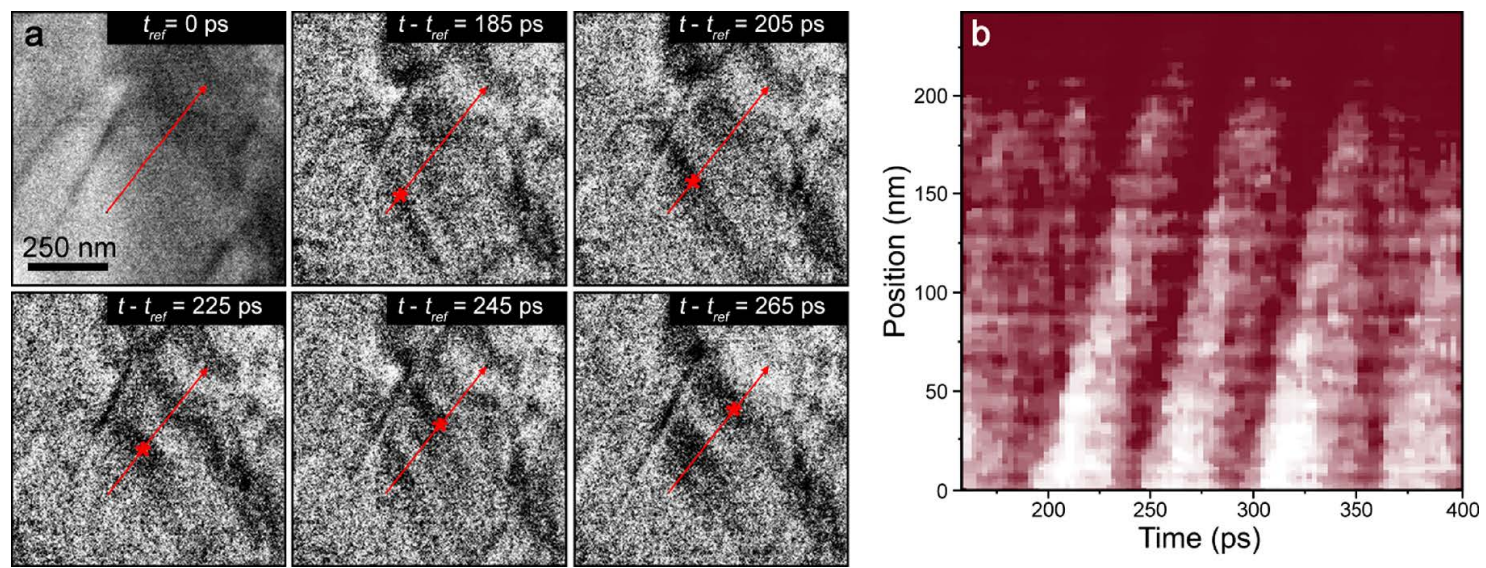

Figure 2.9: Imaging of acoustic phonon propagation in multilayer $1 T$-TaS $\mathbf{T}_{2}$ (A) BF reference image of the specimen at time-zero $\left(t_{\text {ref }}=0 \mathrm{ps}\right)$, and difference images of the observable dynamics. The red star indicates the propagation of an individual phonon wavefront. (B) Space-time plot extracted from a region of interest parallel to the red arrow in A. An average phonon velocity of $4.2(2) \mathrm{nm} / \mathrm{ps}$ can be extracted from the tilted bands. Reprinted with permission from Ref. (61). Copyright 2020 American Chemical Society. 
Furthermore, the nanosecond morphological dynamics of a free-standing black phosphorus membrane has recently been modeled on the basis of structural mechanics simulations and DF images from two different diffraction orders. Due to a high degree of in-plane anisotropy, these two sets of DF images encode very different dynamics along zig-zag and armchair directions of the material (62). For a full reconstruction of the transient strain distribution in graphite, a convergent-beam scanning diffraction technique has proven successful. By scanning a focused beam along the specimen, all relevant diffraction orders can be recorded at once in order to reconstruct the local specimen deformation and visualize propagating strain waves (158).

Beyond these examples of the imaging of morphological dynamics, the real-space mapping of order parameters using UTEM remains to be demonstrated. Typically, the corresponding features are rather weak, as seen in the MIT in $\mathrm{VO}_{2}$ (Fig. 2.7B) or superstructures caused by the presence of CDWs (section 3.2). The convergence angle of the beam in scanning diffraction techniques further expands the low-intensity spots to discs in reciprocal space, making them hard to separate from the background even with current direct detection technology. If convergence angles are too large, overlapping superstructure orders further complicate the evaluation of the individual convergent-beam diffraction patterns (see also section 6.2.6).

When using DF imaging instead to investigate a single-crystalline specimen, one is typically constrained to using an individual circular aperture to tailor the image contrast. Accordingly, intensity of symmetry-equivalent spots also encoding order parameter information is discarded. Taken together with the already weak intensity of these spots and typical beam currents of pulsed electron sources, a sufficient signal level in ultrafast DF images was challenging to achieve prior to the developments reported in Chapter 5. 



\section{Chapter 3}

\section{Properties of transition metal dichalcogenides}

Transition metal dichalcogenides (TMDC) are a class of materials that has been the subject of extensive scientific research since the 1970s. These compounds are described by the chemical formula $\mathrm{MX}_{2}$ with a transition metal $\mathrm{M}$ and a chalcogen $\mathrm{X}$, and form lattice structures consisting of quasi-two-dimensional, covalent $\mathrm{X}-\mathrm{M}-\mathrm{X}$ trilayers as shown in Fig. 3.1A and B. The individual trilayers are bound by weak van-der-Waals interactions between chalcogens along the out-of-plane $c$-axis (179).

As a whole, the TMDC class of materials exhibits an enormous wealth of exotic physical properties due to their unique combination of a quasi-two-dimensional crystal structure, strong spin-orbit and electron-lattice coupling and the occurrence of direct band gaps. External stimuli such as temperature, pressure or electrostatic doping can be used to further tune the material properties (180). Additionally, the properties of TMDCs can be significantly influenced by intercalation, i.e., the introduction of foreign atoms between the layers of the crystal $(11,181)$.

One member of this group is the semiconducting $\mathrm{MoS}_{2}$, which has long been used as a solid lubricant because of the weak interlayer bond (185). Apart from this more practical application, a number of materials in the TMDC family has stimulated scientific interest due to the appearance of correlated phenomena, such as superconductivity and the formation of CDWs in metallic TMDC representatives, e.g. in $\mathrm{TaS}_{2}$ and $\mathrm{NbSe}_{2}(12,186,187)$.

Following the example of the exfoliation of graphene monolayers from graphite (188), monolayers of TMDCs can be obtained from bulk crystals using the same technique (105). As seen in graphene, TMDC monolayers can exhibit new physical properties not present in their bulk ancestors. $\mathrm{MoS}_{2}$ and $\mathrm{WSe}_{2}$, for example, are indirect-band-gap semiconductors in their bulk form, and become direct-band-gap semiconductors when thinned down to a monolayer $(6,189$, 190). This could enable their use in transistors and optoelectronic devices $(6,191,192)$. 
A Perspective rendering of $1 T$-polytype layers
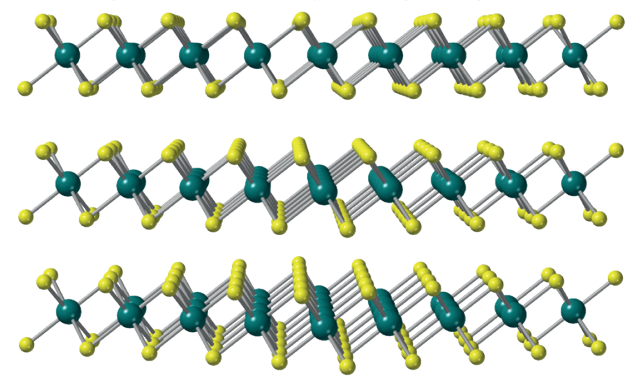

B

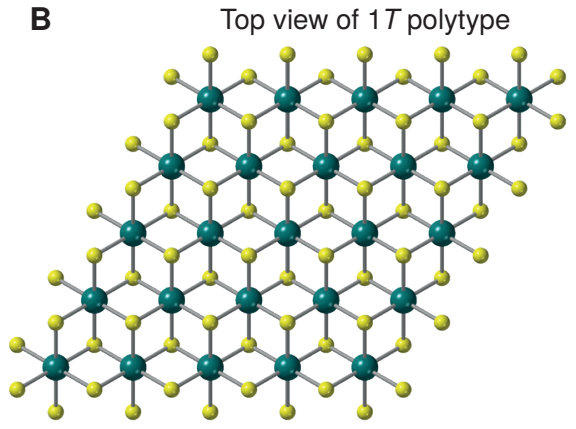

E

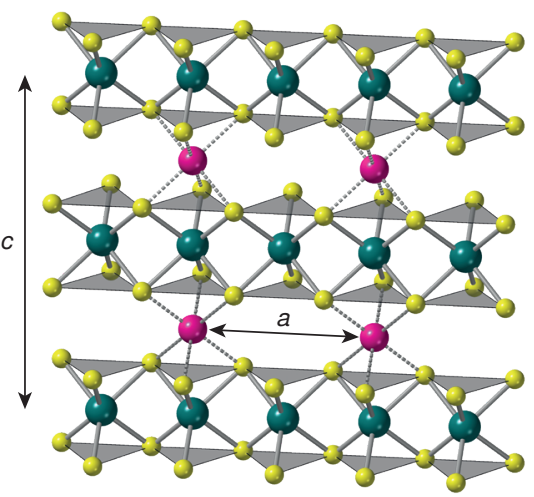

Figure 3.1: Crystal structure of TMDC polytypes. (A) Perspective rendering of three $1 T$-polytype $\mathrm{X}-\mathrm{M}-\mathrm{X}$ trilayers. The transition metal atoms $\mathrm{M}$ are drawn in turquoise, while the chalcogen atoms $\mathrm{X}$ are yellow. (B) Top view of the three trilayers shown in A. The hexagonal arrangement of transition metal and chalcogen atoms is visible. (C) Side view of the structure of a $1 T$-polytype material (space group $P \overline{3} m 1$ ). The in-plane lattice constant $a$ and the out-of-plane lattice constant $c$ are indicated. The octahedral coordination of the transition metal atom is visualized by gray polygons. (D) Side view of the structure of a $2 H_{a}$-polytype material $\left(P 6_{3} / m m c\right)$. The lattice constant $c$ is doubled compared to $\mathrm{C}$, and each unit cell contains two transition metal atoms. Gray polygons visualize the trigonal-prismatic coordination of the transition metal. (E) Side view of the structure of an intercalated $2 \mathrm{H}_{a}$ polytype $\left(\mathrm{Pb}_{3} / \mathrm{mmc}\right)$. Here, the specific case of an $2 \times 2$ in-plane long-range ordering is shown, thus doubling the lattice constant $a$ in comparison to D. The intercalant atom (magenta) is located within the octahedral interstitial site between the $2 H$ layers. The presented atom positions are that of $1 T-\mathrm{TaS}_{2}$ (A to C), $2 \mathrm{H}-\mathrm{TaS}_{2}$ (D) and $\mathrm{Mn}_{0.25} \mathrm{TaS}_{2}$ (E) from Refs. (182-184), but are representative for other TMDCs adopting the same or similar structures.

Additionally, properties depend on the polytype of the TMDC material, the most common of which are $1 T$ and $2 H$. While $1 T$-polytype TMDCs exhibit a trigonal symmetry with one trilayer per unit cell along the crystallographic $c$-axis (Fig. 3.1C), 2H-polytype TMDCs display a double unit cell size in $c$-direction and hexagonal symmetry (Fig. 3.1D ${ }^{4}$ ). The transition metal atoms in $1 T$ and $2 H$ materials are in octahedral and trigonal-prismatic coordination, respectively (179). Thinning a $2 \mathrm{H}$-polytype material down to a monolayer thus breaks inversion symmetry and

${ }^{4}$ It should be noted that the trilayer stacking in the $2 H_{b}$ polytype of $\mathrm{MoS}_{2}$ and $\mathrm{WSe}_{2}$ deviates from the $2 H_{a}$ stacking shown in Fig. 3.1D. However, space group and unit cell size are unaffected. See Ref. (5) for more details, and Ref. (181) for the nomenclature. 

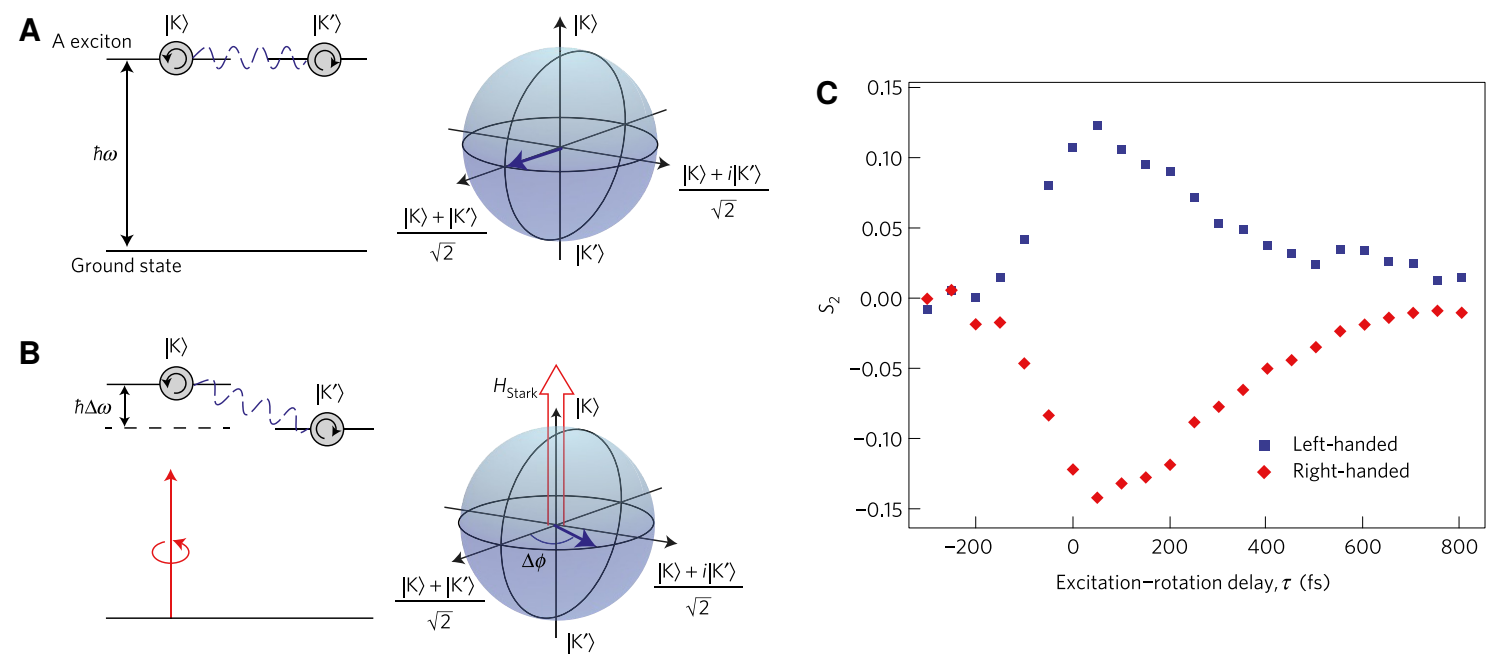

Figure 3.2: Coherent manipulation of valley pseudospin in $\mathbf{W S e}_{2}$. (A) Schematic of the excitation of a coherent superposition of $K$ and $K^{\prime}$ excitons using a linearly polarized optical pulse (left). The fixed phase relationship between the $K$ and $K^{\prime}$ valleys is shown by the pseudospin vector in a Bloch sphere (right). (B) Consecutive interaction with a circularly polarized below-band-gap optical control pulse lifts the valley energy degeneracy by $\hbar \Delta \omega$ due to the optical Stark effect and introduces a phase difference $\Delta \phi \propto \Delta \omega \Delta t$ between the excitons in the $K$ and $K^{\prime}$ valleys, where $\Delta t$ is the duration of the control pulse (left). As a result, the pseudospin is rotated by $\Delta \phi$ (right). (C) When detecting the time-integrated photoluminescence emerging from the material as a function of temporal delay $\tau$ between excitation and control pulse, the normalized Stokes parameter $S_{2}$ characterizes its polarization direction $\left(S_{2}=0\right.$ : no pseudospin rotation; $S_{2}= \pm 1$ : pseudospin rotated by $\left.\pm \pi / 2\right)$. The signal is reversed when switching the helicity of the control pulse (blue and red markers). The decay in $\left|S_{2}\right|$ with increasing delay time indicates an intervalley decoherence time of $350 \mathrm{fs}$. Reprinted by permission from Springer Nature Customer Service Centre GmbH: Springer Nature, Nature Physics, Ref. (23), Copyright 2016 Nature Publishing Group.

leads, together with strong spin-orbit coupling (193), to a valley-selective circular dichroism in monolayer $\mathrm{MoS}_{2}$ and $\mathrm{WSe}_{2}$. The emerging valley degree of freedom shares some similarities with the electron spin, opening up a completely new field of valleytronics $(6,192,194-197)$.

Valley physics allows for intriguing schemes of optical control over charge carriers, as shown by the occurrence of an anomalous Hall effect. This valley Hall effect is enabled by effective magnetic fields with opposite sign experienced by carriers in the $K$ and $K^{\prime}$ valleys of the material, and can be controlled by creating a valley polarization using circularly polarized light (198). Furthermore, it has been shown that a coherent superposition between $K$ and $K^{\prime}$ excitons can be manipulated by the helicity of optical pulses with photon energies below the band gap of the material (Fig. 3.2). The properties of the emerging photoluminescence help to understand valley relaxation and decoherence mechanisms, paving the way for the use of the valley degree of freedom as an information carrier $(23,199)$.

The consequences of the loss of inversion symmetry are just one example of how the quasitwo-dimensionality of TMDCs leads to the occurrence of exotic phenomena. This thesis presents 
two studies on materials whose functionality is also enabled by dimensionality: intercalation of $3 d$ transition metals between the layers of a $2 \mathrm{H}-\mathrm{TaS}_{2}$ host lattice gives rise to ferromagnetic phases at low temperatures (Chapter 4), and the low-dimensional Fermi surface of $1 T-\mathrm{TaS}_{2}$ offers ideal conditions for the emergence of a number of different CDW phases (Chapter 5). In the following sections, the relevant physical basics and material properties are outlined and put into context with previous works on these materials.

\subsection{Charge-density wave formation and metal-insulator transitions}

Various TMDCs in general, and $1 T-\mathrm{TaS}_{2}$ specifically, exhibit one or more CDW phases. In the presence of a CDW, the spatial electron density is modulated to form a standing-wave pattern. A periodic lattice distortion (PLD) accompanies the CDW due to strong electronphonon interactions, forming a superlattice that is either commensurate $(\mathrm{C})$ or incommensurate (IC) with the underlying structural lattice.

The instability of a one-dimensional chain of atoms to the formation of a CDW was first predicted by Rudolph Peierls $(160,200)$. Starting from the undistorted chain in Fig. 3.3A and following the descriptions in Ref. (201), the energy gain connected to CDW/PLD formation in this system can be demonstrated. Assuming a pure sinusoidal modulation of the electron density

$$
\rho(\vec{r})=\rho_{0}(\vec{r})\left[1+\rho_{1} \cos (2 \pi \vec{Q} \cdot \vec{r}+\varphi)\right],
$$

where $\rho_{0}$ is the electron density of the undistorted state, and $\rho_{1}, \vec{Q}$ and $\varphi$ are the amplitude, wave vector and phase of the modulation, respectively. The movement of the atoms due to the coupled PLD can by described by

$$
\vec{u}(\vec{r})=\vec{A} \sin (2 \pi \vec{Q} \cdot \vec{r}+\varphi) .
$$

The norm of the amplitude $\vec{A}$ is generally small in comparison to the lattice constant $a$. Both modulations are schematically depicted in the top panel of Fig. 3.3B. From the model, we obtain the following stability criterion for CDW/PLD formation via a statically displaced phonon mode $\vec{k}$ in a weak-coupling limit:

$$
\frac{4 g_{\vec{k}}^{2}}{\hbar \omega_{\vec{k}}}>\frac{1}{\chi(\vec{k})} .
$$

Here, $g_{\vec{k}}$ is the electron-phonon coupling constant, $\omega_{\vec{k}}$ is the phonon frequency, and $\chi(\vec{k})$ is the electronic susceptibility. For a Peierls instability to occur, a large coupling constant and 
A

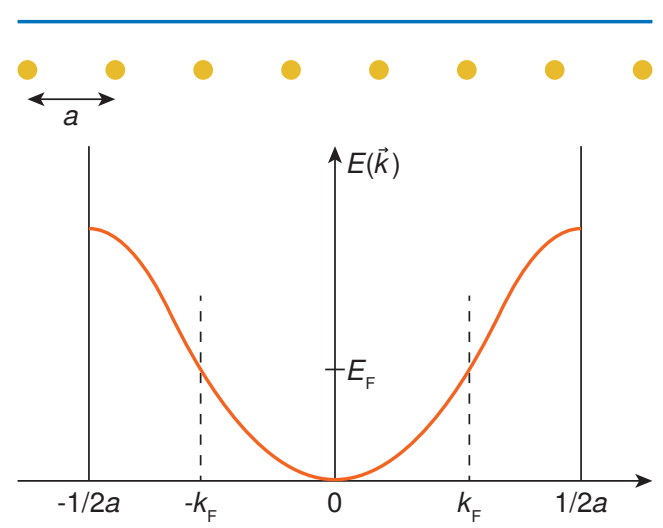

$\rho(\vec{r})$
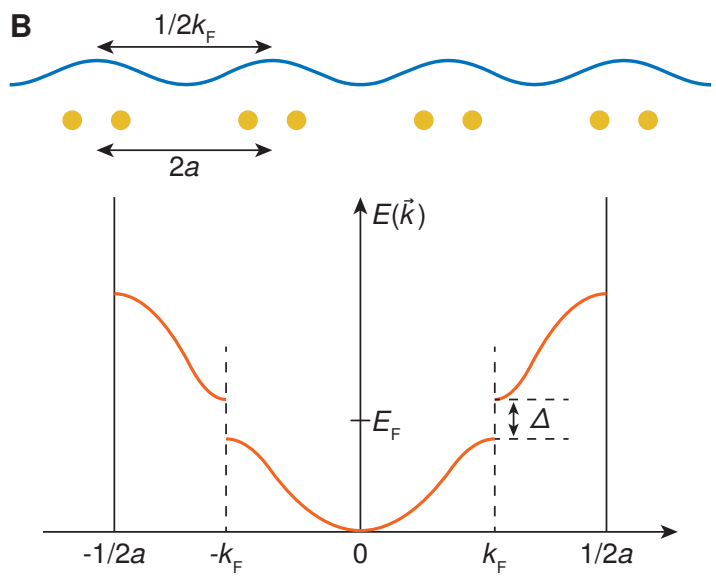

Figure 3.3: Peierls instability in a one-dimensional chain of atoms. (A) Schematic view of the undistorted chain of atoms (yellow) with lattice constant $a$ and isotropic electron density $\rho(\vec{r})$ (blue). The conduction band (orange) is half-filled up to the Fermi energy $E_{\mathrm{F}}$. (B) Below the transition temperature $T_{\mathrm{C}}$, both electron density and atom positions are modulated. The periodicity of the CDW/PLD state is $2 a$. Gaps of $\Delta$ open at the Fermi energy, resulting in a filled valence band and an empty conduction band. Adapted from Ref. (160).

a large susceptibility are required at the CDW wave vector $\vec{Q}=\vec{k}$. For the one-dimensional chain, these conditions are fulfilled at $2 \vec{k}_{\mathrm{F}}$, where $\vec{k}_{\mathrm{F}}$ is the Fermi wave vector. With falling temperatures, $\chi\left(2 \vec{k}_{\mathrm{F}}, T\right)$ diverges which causes a renormalization of phonon frequencies around $\vec{Q}=2 \vec{k}_{\mathrm{F}}$. This Kohn anomaly leads to a complete softening of the phonon mode $\vec{Q}$ (i.e., the renormalized phonon frequency $\tilde{\omega}_{\vec{Q}}$ approaches zero) and a second-order phase transition at the CDW transition temperature $T_{\mathrm{C}}$. The CDW wavelength is given by $\lambda=1 / 2 \vec{k}_{\mathrm{F}}(160,201)$.

In the presence of the CDW/PLD, the Brillouin zone edges of the superstructure coincide with the Fermi points at $\pm \vec{k}_{\mathrm{F}}$, opening an energy gap at the Fermi energy $E_{\mathrm{F}}$ (Fig. 3.3B, bottom). The gap size $\Delta$ is related to the PLD amplitude $\vec{A}_{\vec{Q}}$, the coupling constant $g_{\vec{Q}}$, the unrenormalized phonon frequency $\omega_{\vec{Q}}$ and the ionic mass $M$ of the atom species:

$$
\Delta=2\left|\vec{A}_{\vec{Q}}\right| g_{\vec{Q}} \sqrt{\frac{2 M \omega_{\vec{Q}}}{\hbar}} .
$$

The lower branch of the energy dispersion is completely occupied while the upper branch is unoccupied. The CDW/PLD formation in a linear chain therefore results in the occurrence of a MIT. Although, in this very specific case, the CDW wave vector $\vec{Q}$ is commensurate with the underlying one-dimensional lattice, a Peierls CDW generally is incommensurate due to the dependency of $\vec{Q}$ on the electron filling of the band structure (201).

The divergence of the susceptibility $\chi(\vec{k})$ at $2 \vec{k}_{\mathrm{F}}$ stems from a particular topology of the Fermi surface which allows for perfect nesting. Pairs of states at the Fermi energy $E_{\mathrm{F}}$ which 
A

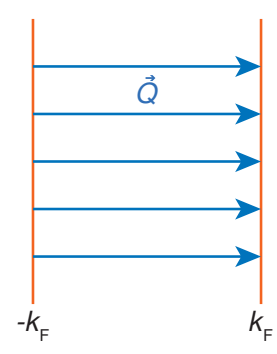

Perfect nesting in 1D
B

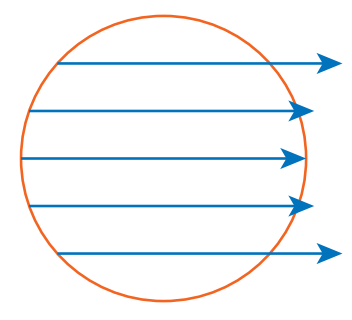

No nesting in 2D
C

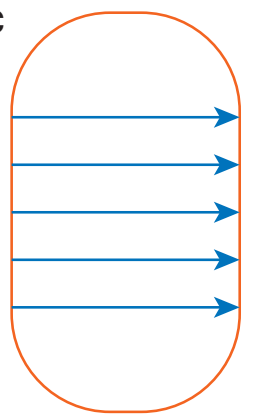

Partial nesting in 2D

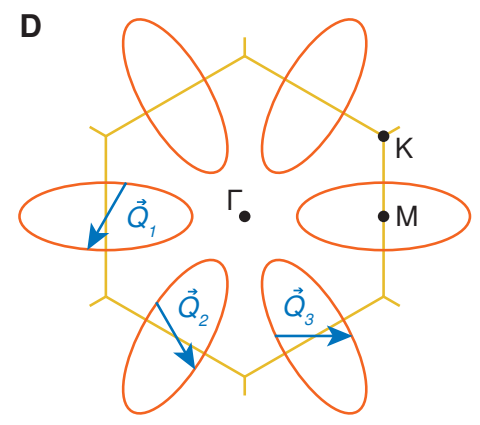

Fermi surface of $1 T-\mathrm{TaS}_{2}$

Figure 3.4: Fermi surface nesting in one- and two-dimensional cases. (A) Fermi surface topology of a onedimensional free-electron gas. The nesting vector $\vec{Q}$ (blue), connecting the Fermi surface at $\pm \vec{k}_{\mathrm{F}}$ (orange), is indicated. (B) Fermi surface topology of a two-dimensional free-electron gas. In this case, there are no pairs of states that allow for nesting. A and B adapted from Ref. (160). (C) The Fermi surface topology of a quasi-two-dimensional material allows for partial nesting. Adapted from Ref. (202). (D) Schematic of the Fermi surface of $1 T-\mathrm{TaS}_{2}$ in the first Brillouin zone (yellow) as an example of a quasi-two-dimensional material. The nesting vectors for the triple IC CDW in the material are indicated. Adapted from Ref. (203).

differ by $2 \vec{k}_{\mathrm{F}}$ give a diverging contribution to the integral describing $\chi(\vec{k})(160)$. These nesting conditions are trivially fulfilled in the complete Fermi surface of the one-dimensional chain. In higher-dimensional Fermi surfaces, however, perfect Fermi surface nesting conditions are typically only present, if at all, in low-dimensional materials and in a small fraction of the Fermi surface (Fig. 3.4).

CDWs exhibit two types of collective excitations. Amplitudons are oscillations of the CDW and PLD amplitudes $\rho_{1}$ and $\vec{A}$, while phasons are collective fluctuations of the phase $\varphi$ (160, 204). The $\vec{k}=0$ limit of the phason corresponds to a translation of the CDW through the lattice. However, at zero applied field, a number of effects such as commensurability with the underlying lattice, three-dimensional ordering or impurities practically always lead to a local pinning of the CDW phase $(203,205,206)$.

Another mechanism relevant in $1 T-\mathrm{TaS}_{2}$ that can lead to a gap opening in the electronic dispersion relation is driven by electron-electron correlations, as opposed to the CDW gap driven by electron-phonon coupling. This is described by the Hubbard model which has been introduced to explain correlation effects in $3 d$ transition metals (207). Some materials that would be conductors following conventional band theory show insulating behavior instead-they are Mott insulators.

To describe this behavior, the single-band Hubbard model introduces the parameter $U$ describing the Coulomb interaction energy of two electrons on the same atom, a hopping amplitude $t$ which is proportional to the band width $w$, and the degree of band filling $n$. In the strong-coupling 
limit $U \gg t$, the band splits into a lower Hubbard band and an upper Hubbard band. Assuming a half-filled band, the material would show insulating behavior due to Mott localization (208). In this model, a MIT - the Mott-Hubbard transition — can be initiated by changing the model parameters, e.g., via external pressure, doping or temperature $(159,209)$.

There are two additional scenarios that are worth mentioning in the context of MITs. Anderson localization can lead to a disorder-induced MIT and plays some role in the C phase of $1 T-\mathrm{TaS}_{2}$, as we will see later (210). In some other TMDCs, such as $1 T-\mathrm{TiSe}_{2}$, there are indications of a significant excitonic contribution to the insulating ground state. An excitonic insulator is formed when the band gap in a semiconductor is smaller than the exciton binding energy. This leads to a purely electronic instability (in contrast to the Peierls instability which is a combined electron-lattice instability). As a result, a spin-density wave or a CDW is formed which may be, in case of a CDW, accompanied by a PLD as a byproduct due to electron-phonon coupling (201).

\subsection{Charge-density wave signatures in electron diffraction}

CDW formation creates a unique signature in electron diffraction patterns. As outlined in section 2.3, electron diffraction is sensitive to the distribution of charges in the crystal unit cell, i.e., mainly to the charge of the nuclei and core electrons. The CDW itself, however, manifests itself in a redistribution of conduction electrons (203). This means that electron diffraction only shows the PLD accompanying the CDW formation, but not the electronic CDW itself.

The structure factor for a sinusoidal modulation of atom positions $\vec{u}_{i}=\vec{A} \cdot \sin \left(2 \pi \vec{Q} \cdot \vec{r}_{i}\right)$ (neglecting the phase $\varphi$ from Eq. 3.2) in the linear chain of atoms has been described by Overhauser (204). Including the PLD-induced atomic displacements $\vec{u}_{i}$, the structure factor (Eq. 2.3) assumes the form

$$
\begin{aligned}
F(\vec{q}) & =\sum_{i} f_{i}(\vec{q}) \exp \left[2 \pi i \vec{q} \cdot\left(\vec{r}_{i}+\vec{u}_{i}\right)\right] \\
& =\sum_{i} f_{i}(\vec{q}) \exp \left[2 \pi i \vec{q} \cdot\left(\vec{r}_{i}+\vec{A} \cdot \sin \left(2 \pi \vec{Q} \cdot \vec{r}_{i}\right)\right)\right]
\end{aligned}
$$

With the Jacobi-Anger identity (211)

$$
\exp (i x \cos \theta)=\sum_{n=-\infty}^{\infty} i^{n} J_{n}(x) \exp (i n \theta)
$$

where $J_{n}$ is the $n$-th Bessel function of the first kind, and the substitution $\theta \rightarrow \theta-\pi / 2$, we 
obtain

$$
F(\vec{q})=\sum_{i} f_{i}(\vec{q}) \sum_{n=-\infty}^{\infty} J_{n}(2 \pi \vec{Q} \cdot \vec{A}) \exp \left[2 \pi i(\vec{q}+n \vec{Q}) \cdot \vec{r}_{i}\right] .
$$

This expression leads to a non-vanishing $F(\vec{q})$ not only for the case where $\vec{q}$ is a reciprocal lattice vector $\vec{q}_{h k l}$ (Bragg reflections of the structural lattice), but also to the appearance of orders $n$ of CDW superstructure reflections with reciprocal lattice vectors $\vec{q}=\vec{q}_{h k l}-n \vec{Q}$. CDW spot intensities are determined by Bessel functions. In the special case of a commensurate CDW, Eq. 3.8 describes the interference between different orders of Bragg and CDW spots, leading to a complicated dependency of CDW spot intensities on the scattering vector $\vec{q}$.

For a triple CDW as shown in Fig. 3.4D, with periodicities $\vec{Q}_{j}$ and amplitudes $\vec{A}_{j}$, the corresponding expression is:

$$
\begin{gathered}
F(\vec{q})=\sum_{i} f_{i}(\vec{q}) \sum_{n_{1}, n_{2}, n_{3}} J_{n_{1}}\left(2 \pi \vec{Q}_{1} \cdot \vec{A}_{1}\right) J_{n_{2}}\left(2 \pi \vec{Q}_{2} \cdot \vec{A}_{2}\right) J_{n_{3}}\left(2 \pi \vec{Q}_{3} \cdot \vec{A}_{3}\right) \\
\cdot \exp \left[2 \pi i\left(\vec{q}+\sum_{j=1}^{3} n_{j} \vec{Q}_{j}\right) \cdot \vec{r}_{i}\right]
\end{gathered}
$$

Due to hexagonal symmetry, the modulation wave vectors are related via $\vec{Q}_{3}=\vec{Q}_{2}-\vec{Q}_{1}$, such that an infinite number of different triples $\left(n_{1}, n_{2}, n_{3}\right)$ leads to the same superstructure reflection $\vec{q}$ and an even more complicated CDW intensity behavior than in the one-dimensional case.

\subsection{Order parameters and phenomenological Landau theory}

As motivated in the introduction of the present thesis, order parameters are a useful concept to describe the state of a system as a function of position and time. On the phase diagram, two phases of matter with different symmetry are separated by a line of transitions, reflecting the fact that symmetry cannot change continuously. While the order parameter is zero in one phase (e.g., above a critical temperature $T_{\mathrm{C}}$ ), it assumes a non-zero value when crossing the transition line towards a symmetry-broken, ordered phase. The choice of order parameter for a given system is not unique and any thermodynamic variable that follows the aforementioned behavior may be selected. While order parameters can be scalar values, they may also take the form of a complex number or a tensor (212).

According to phenomenological Landau theory, one can write down a free-energy functional $\mathscr{L}(\phi, T)$ of any system as a function of order parameter $\phi(\vec{r})$ and temperature $T$. A suitable 
functional to describe a scalar, coarse-grained order parameter field is $(212,213)$ :

$$
\mathscr{L}(\phi, T)=\int \mathrm{d}^{d} x\left[\frac{1}{2}(\nabla \phi)^{2}+F(\phi, T)\right] .
$$

The potential $F$ is the Landau free-energy density and $d$ is the dimensionality of the system. The free energy must be analytic and consistent with the symmetries of the system (212). The time evolution of a non-conserved order parameter can be calculated using a dissipative equation of motion which is often referred to as time-dependent Ginzburg-Landau model ${ }^{5}$ (213, 214):

$$
\frac{\mathrm{d} \phi}{\mathrm{d} t}=-\frac{\partial \mathscr{L}}{\partial \phi}
$$

Inserting $\mathscr{L}$ yields (213):

$$
\frac{\mathrm{d} \phi}{\mathrm{d} t}=\Delta \phi-\frac{\partial F}{\partial \phi} .
$$

Near the critical temperature, $F$ can be expanded in a power series in $\phi(212)$ :

$$
F(\phi, T)=\sum_{n=0}^{\infty} \alpha_{n}(T) \phi^{n}
$$

An exemplary choice of an order parameter is the magnetization $M$ in the ferromagnetic Ising model (thus setting $\phi=M$ in the above equations). As pointed out by ERNST IsING, the one-dimensional chain of interacting spins does not exhibit a phase transition (215). Later on, the field-free two-dimensional model was solved analytically and shown to spontaneously magnetize below a critical temperature (216). To describe the ferromagnetic Ising model, it is sufficient to expand Eq. 3.13 to $\mathcal{O}\left(M^{4}\right)(212)$ :

$$
F(M, T)=F_{0}+\alpha_{0} \cdot\left(T-T_{\mathrm{C}}\right) \cdot M^{2}+\frac{\beta}{2} \cdot M^{4}-H M
$$

The coefficients $\alpha_{0}>0$ and $\beta>0$ are phenomenological parameters and $H$ is an additional external magnetic field.

At zero field and for $T<T_{\mathrm{C}}$, Eq. 3.14 describes a double-well potential with minima corresponding to both possible directions of the magnetization (Fig. 3.5A). The closer $T$ gets to $T_{\mathrm{C}}$, the more the positions of both minima approach $M=0$ until the minima merge at $T=T_{\mathrm{C}}$. Above the phase transition temperature, the potential is parabolic in shape with a single minimum

\footnotetext{
${ }^{5} \mathrm{By}$ adding a Laplacian term $-\Delta$ in front of the right-hand side of Eq. 3.11, the model can be modified to describe conserved order parameter fields. This is relevant, for example, when modeling phase separation in binary alloys $(213,214)$.
} 

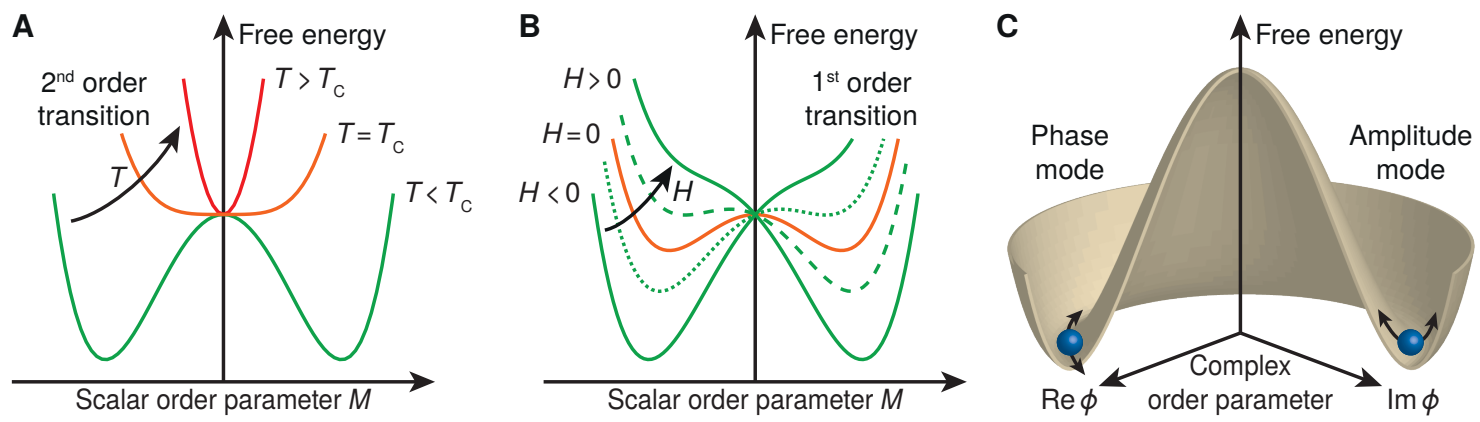

Figure 3.5: Landau free-energy densities for the Ising model and a complex order parameter. (A) Free energy for the ferromagnetic Ising model at three different temperatures below, at and above the critical temperature $T_{\mathrm{C}}$ (green, orange and red curve, respectively). With increasing temperature, both minima of the symmetry-broken phase continuously approach $M=0$ until they merge at $T_{\mathrm{C}}$. (B) Free energy for the Ising model at different external fields $H$ and $T<T_{\mathrm{C}}$. At $H=0$, the double-well potential from A is reproduced (orange curve). Green curves show the shape of the potential for applied fields $H \neq 0$ (solid line: monostable potential; broken line: bistable potential). (C) "Sombrero" potential of a complex order parameter $\phi$. Blue spheres schematically indicate amplitude and phase modes.

at $M=0$, reflecting the loss of ferromagnetism. This is consistent with a second-order phase transition. Different behavior is observed when changing the external field $H$ instead of the temperature. In this case, the energetic degeneracy of the potential minima is lifted and the system exhibits a preferred direction of magnetization as well as hysteresis, a sign of a first-order phase transition (Fig. 3.5B).

The potential term $\partial F / \partial M$ in Eq. 3.12 causes the magnetization to locally relax to the respective potential minimum with the passage of time. Simultaneously, the Laplacian term $\Delta M$ describes the energy cost related to the formation of a domain wall between two regions of opposite magnetization and drives the spatial evolution of magnetic domains the system (213). The phenomenological parameters $\alpha_{0}$ and $\beta$ and the critical temperature $T_{\mathrm{C}}$ can be derived from microscopic theory (216). The fact that an analytical solution exists for the two-dimensional Ising model, however, is a rather rare occasion. Alternatively, one can use the shape of the potential (Eq. 3.14) to derive relationships between the parameters and thermodynamic observables such as specific heat and magnetic susceptibility (217).

Due to its universality, this concept can be applied to describe the properties and nonequilibrium dynamics of other symmetry-breaking phase transitions as well. This includes the emergence of superconductivity (218) and the formation of CDWs (203). In the latter case, either the modulation of the conduction electron density (Eq. 3.1) or of the atomic positions (Eq. 3.2) can be considered natural order parameters of a one-dimensional CDW. For the triple CDW in hexagonal TMDCs (see also Fig. 3.4D), McMillan defined the real order parameter $\phi(\vec{r})$ as a sum of three complex order parameters $\phi_{i}(\vec{r})$ representing the individual 
CDW components $\vec{Q}_{i}$ :

$$
\phi=\operatorname{Re}\left[\phi_{1}+\phi_{2}+\phi_{3}\right]
$$

McMillan's free energy is then formulated in terms of $\phi_{i}$ and $\vec{Q}_{i}$ to create a potential that favors a triple CDW with its wavelength and direction given by the modulation wave vectors. The coupling between the three CDWs is controlled by a phasing term, ensuring that the phases of all $\phi_{i}$ add up to multiples of $2 \pi$ in thermodynamic equilibrium $(203,219)$.

Neglecting commensurability effects, the potential of a complex order parameter can be visualized in a "Sombrero" shape (Fig. 3.5C). For a C CDW, the angular dependency is more complex (220). Amplitude and phase modes of the CDW (section 3.1) are fluctuations along the radial and azimuthal coordinates of the potential, respectively. The emergence of these modes is dictated by the spontaneous symmetry breaking. In analogy to the theory of superconductivity, both excitations are sometimes called Higgs and Nambu-Goldstone modes (221).

In the past, Ginzburg-Landau models with different formulations of the free energy have been applied to describe CDWs and reproduce experimental observations. Static phenomena can be modeled by minimizing a suitable Landau free-energy functional $(26,46)$. In the time domain, the simulation of topological defects in a nascent IC CDW phase has been realized by calculating the evolution of the order parameter using Eq. 3.11, starting from a disordered state (222).

For modeling the reversible transition between two phases, one needs to consider the (mostly optical) experimental stimulus as a perturbation term in the Landau free energy. This term is typically quadratic in the order parameter and has the same effect on the shape of the potential as the temperature-dependent term in the Ising model (Eq. 3.14). The optical excitation quenches the symmetry-broken low-temperature order and transiently returns the system to a high-symmetry state. However, oscillations of the amplitude mode are not reproduced by the dissipative GinzburgLandau model (Eq. 3.11). In order to achieve this, a second time derivative can be added to the equation of motion (223-229).

Depending on the specific experimental conditions, the external stimulus may not only transiently perturb the potential on a femto- to picosecond time scale but deposit a significant amount of heat in the system that dissipates only after nano- to microseconds. We will show in Chapter 5 that, in this case, the spatiotemporal evolution of a CDW system can be simulated by coupling the free-energy density to the temperature of the electron system via the quadratic term $(228,229)$. The excitation is then modeled in terms of an instantaneous increase in electronic temperature. The electron system cools by transferring energy to the lattice and, subsequently, out of the simulation volume (see section 5.2.11 for details). 


\subsection{Equilibrium phase diagram of $1 T-\mathrm{TaS}_{2}$}

The CDW compound $1 T-\mathrm{TaS}_{2}$ is one of the most-studied TMDCs due to its intricate phase diagram with various different CDW and superconducting phases, some of which are present at and even above room-temperature. At these temperatures, however, $2 \mathrm{H}-\mathrm{TaS}_{2}$ is the stable modification of $\mathrm{TaS}_{2} .1 T-\mathrm{TaS}_{2}$ is only stable at high temperatures above $780 \mathrm{~K}$ and can be retained as a metastable polytype by thermal quenching to room-temperature (230). Temperatures between $780 \mathrm{~K}$ and $550 \mathrm{~K}$ are difficult to study because the material transforms back to the stable $2 H$ polytype $(12,231)$. The lattice constants of the undistorted metallic high-temperature phase of $1 T-\mathrm{TaS}_{2}$ are $a=3.36 \AA$ and $c=5.90 \AA$ (232). The corresponding crystal structure is shown in Fig. 3.1A to C.
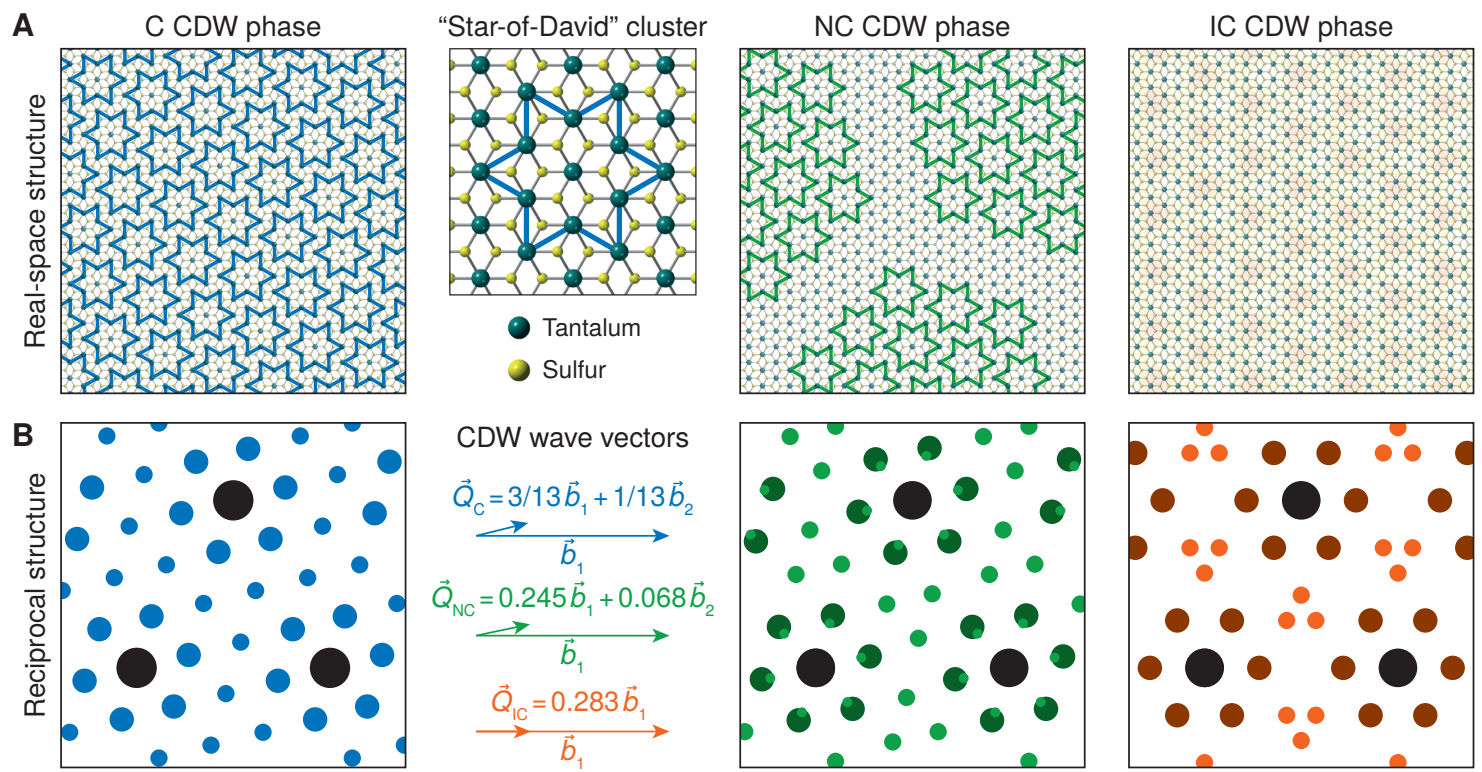

C

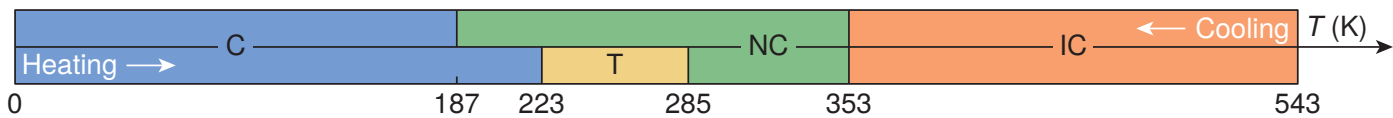

Figure 3.6: Phase diagram, real-space structure and reciprocal structure of the CDW phases in $1 T$-TaS . $_{2}$.

(A) Real-space structure of the C phase, the NC phase and IC phase. The $\sqrt{13} \times \sqrt{13}$ superstructure of the $\mathrm{C}$ CDW leads to the formation of "Star-of-David" clusters, consisting of 13 tantalum atoms each. In these clusters, the outer 12 tantalum atoms contract towards the central atom. In the NC phase, $\mathrm{C}$ domains are separated by discommensurations of the IC type. In the IC phase, the CDW is not registered with the lattice as indicated by the incommensurate charge-density modulation in the image background. (B) Reciprocal space structure of the three CDW phases along with reciprocal lattice and modulation wave vectors (233). The other vectors can be obtained by rotating in increments of $60^{\circ}$. Structural and superstructure spots are drawn in black and color, respectively. Superstructure spot size indicates the diffraction order [large: $1^{\text {st }}$ order; medium: $2^{\text {nd }}$ order; small (NC phase only): $6^{\text {th }}$ order]. Spots drawn in darker shades lie outside of the $q_{z}=0$ plane. (C) Phase diagram of the thermodynamically stable CDW phases in $1 T-\mathrm{TaS}_{2}$. 
When cooling the material below a temperature of $543 \mathrm{~K}, 1 T-\mathrm{TaS}_{2}$ forms an IC CDW that is aligned with the atomic lattice. Below $353 \mathrm{~K}$, the material undergoes a first-order phase transition and rearranges into a nearly commensurate (NC) CDW state. This is a specific case of an IC CDW with in-plane modulation wave vectors that are very close to being commensurate with the underlying lattice. Depending on the exact temperature within the NC CDW stability regime, angles in a range of $11^{\circ}$ to $13^{\circ}$ between the modulation vectors and the structural lattice are observed. Below a temperature of $183 \mathrm{~K}$, another first-order phase transition leads to the formation of a low-temperature C CDW at an angle of $13.9^{\circ}(182,231,233)$. In both, the NC and $\mathrm{C}$ phases, the in-plane mirror symmetry is broken due to the non-zero angle between the modulation and lattice vectors. This leads to the possible formation of symmetry-equivalent mirror phases called $\alpha$ and $\beta$ configurations (see also section 6.2.3).

The situation observed when heating $1 T-\mathrm{TaS}_{2}$ from the $\mathrm{C}$ phase is even more complex. Besides a hysteresis in the transition temperatures, a triclinic (T) CDW phase appears between $223 \mathrm{~K}$ and $283 \mathrm{~K}$. This phase loses its hexagonal symmetry due to a striped domain structure $(231,234)$. A schematic of the phase diagram for heating and cooling cycles is shown in Fig. 3.6C (231).

The C CDW and the IC CDW represent two extreme cases of CDW formation. In the C phase, the commensurate modulation leads to the formation of a $\sqrt{13} \times \sqrt{13}$ superstructure. In this superstructure, clusters of 13 tantalum atoms each contract towards the central atom (Fig. 3.6A, left panel). While the tantalum atoms are displaced in-plane by up to $7 \%$ of the lattice constant, a comparably large value, the sulfur atom modulation is mainly directed out-of-plane (235). The stacking of the C CDW in out-of-plane direction is partially disordered (73, 236-238). In reciprocal space, the PLD of the C CDW leads to superstructure reflections of first and second order as indicated by the blue spots in Fig. 3.6B (233). As a commensurate CDW, the Peierls model for a weak-coupling CDW is not sufficient to describe this type of electron-lattice interaction. On the contrary, the $\mathrm{C} \mathrm{CDW}$ is a prototypical example of a strong-coupling CDW in which the electronic energy gain is not concentrated near $k_{\mathrm{F}}$ but spread over the band structure as can be seen in Fig. 3.7 (201).

The insulating behavior of the $\mathrm{C}$ phase is still subject of active investigation. It has early been attributed to the involvement of electron correlation effects in the NC/C phase transition, leading to a Mott-Hubbard-type transition. Due to overlapping Hubbard bands, a pseudo gap at the Fermi level $E_{\mathrm{F}}$ is formed (i.e., the energy gap does not span the full Brillouin zone). The occurrence of Anderson localization then further prevents metallic conductivity (240). Time-resolved photoemission experiments have proven that Mott localization certainly plays an important role for the C phase of $1 T-\mathrm{TaS}_{2}(241-244)$. Specifically, Mott-insulating properties could lead to the 


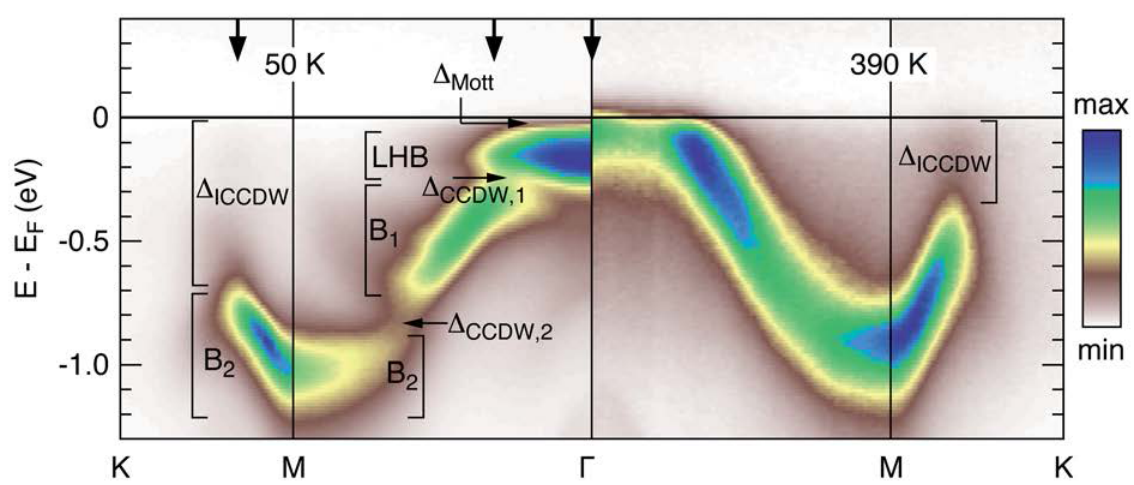

Figure 3.7: Static angle-resolved photoemission spectra of the $\mathrm{C}$ and $\mathrm{IC}$ phase in $\mathbf{1 T}$-TaS $\mathrm{T}_{\mathbf{2}}$. The characteristic gaps in the band structure at $50 \mathrm{~K}$ (C phase) and at $390 \mathrm{~K}$ (IC phase) are indicated. Both phases exhibit the Peierls-induced IC CDW gap at the Fermi energy $E_{\mathrm{F}}$. Additional C CDW gaps and the Mott gap split the band structure of the $\mathrm{C}$ phase into three submanifolds $B_{1}, B_{2}$ and the lower Hubbard band (LHB). The presence of the $\mathrm{C}$ CDW gaps visualizes that the electronic energy gain in the $\mathrm{C}$ phase is distributed over the Brillouin zone (201). Reprinted from Ref. (239). Licensed under CC BY 3.0.

formation of a quantum spin liquid in the $\mathrm{C}$ phase (245). However, recent findings also indicate that interlayer dimerization possibly leads to a band insulating state without the need to invoke electron-electron correlations $(238,246)$. It has been proposed to split the $\mathrm{C}$ phase in the phase diagram in a band-insulating $\mathrm{C}$ phase and a Mott-insulating I phase that only occurs during a heating cycle (247). Most recent calculations were finally able to reproduce the Mott-insulating nature of the $\mathrm{C}$ phase without assuming a double-layer order (248).

The drastic difference between the properties of the $\mathrm{C}$ and the IC phase can also be seen in electrical resistivity measurements (black curve in Fig. 3.8). The IC phase exhibits a resistivity that is multiple orders of magnitude smaller than that of the $\mathrm{C}$ phase. Additionally, the mechanism behind the formation of the IC CDW is much closer to a nesting-induced Peierls CDW (239). The loss in lattice registration is also reflected in the collapse of the Ta $4 f$ core-level splitting observed in $\mathrm{C}$ phase photoemission experiments as shown in red in Fig. 3.8 (249). The reciprocal structure of the IC PLD is depicted in the right panel of Fig. 3.6B up to the second-order superstructure reflections. The first-order spots are faint or invisible in zone-axis TEM diffraction patterns. This is due to a three-fold out-of-plane stacking periodicity of the IC CDW. Second-order superstructure spots can be seen in Fig. 5.5. In surface-sensitive techniques such as low-energy electron diffraction (LEED), however, first-order spots are the dominant feature due to a reduced out-of-plane probing depth $(222,250)$.

The NC phase, finally, exhibits a peculiar structure combining features of the $\mathrm{C}$ and IC phase. Distorted hexagonal domains of the $\mathrm{C}$ type are separated by discommensurations of the IC type, where the CDW amplitude is suppressed (Fig. 3.6A, center panel). The discommensurations 


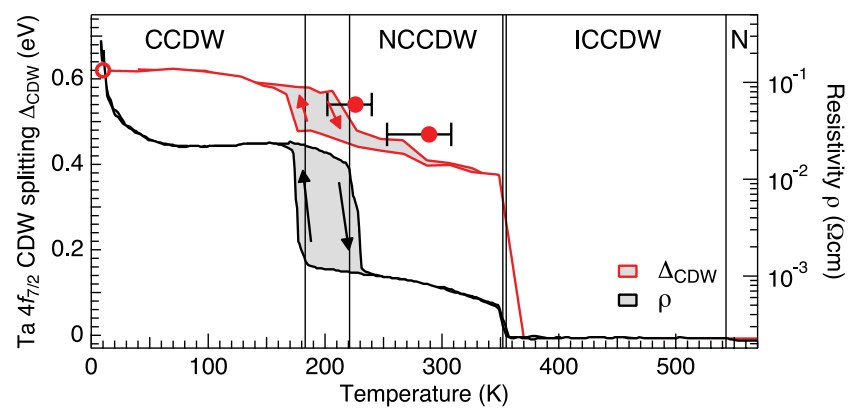

Figure 3.8: Electrical resistivity and core-level splitting in $\mathbf{1 T}-\mathrm{TaS}_{\mathbf{2}}$. Temperature-dependent measurements of the electrical resistivity (black) and the Ta $4 f$ core-level splitting $\Delta_{\mathrm{CDW}}$ (red) indicate the different electrical properties and orderings of the $\mathrm{C}, \mathrm{NC}$ and IC phases. The core-level splitting reflects the inequivalent Ta sites in the "Star-of-David" clusters. Reprinted with permission from Ref. (249). Copyright 2010 by the American Physical Society.

are not of constant width, but form a pattern of corner-sharing triangles. This can be described using a block-wave approach by taking a large number of higher-harmonic contributions to the fundamental wave vector $\vec{Q}_{\mathrm{NC}}$ into account. While the phase of the modulations is flat within the domains, phase jumps occur in the discommensurations. In the NC phase, the in-plane tantalum displacement is up to $9 \%$ of the lattice constant - even larger than in the $\mathrm{C}$ phase $(182$, 219). The lower the temperature, the larger the C-type domains within in the NC phase become. While $70 \AA$ domain sizes are observed in the bulk material at room temperature, domain sizes approach $100 \AA$ close to the NC/C phase transition temperature. This behavior leads to the broad angular regime given for the modulation wave vector and is further modified in the case of few-nanometer specimen thicknesses $(9,231)$. The discommensuration network around the C-type domains is understood to host the metallic conductivity of the NC phase (9).

In reciprocal space, the nearly commensurate characteristics of the NC phase leads to the appearance of higher-order superstructure spots that lie close to lower-order reflections. The center panel of Fig. 3.6B shows this situation for first- and sixth-order superstructure reflections. In zone-axis TEM diffraction patterns, mainly second- and sixth-order spots are visible due to three-fold out-of-plane stacking (as it is the case for the IC phase) (182).

Apart from the thermodynamically stable CDW phases that are shown in the phase diagram in Fig. 3.6C as a function of temperature, further phases can be realized as a function of additional tuning parameters, such as intercalation (section 3.7) and choice of substrate (251). Slight iron doping can lead to the emergence of an Anderson-localized phase and a superconducting phase at low temperatures, while titanium substitution strongly modifies the CDW phase diagram (7, $8)$. Increasing pressure does not only considerably shift the transition temperatures between the CDW phases but leads to a superconducting state as well (252). This rich electronic and structural 
phase diagram of $1 T-\mathrm{TaS}_{2}$ provides many opportunities to study the various electron-electron and electron-lattice correlations in a controlled way.

\subsection{Ultrafast charge-density wave dynamics in $1 T-\mathrm{TaS}_{2}$}

Static x-ray and electron diffraction $(12,233)$, scanning tunneling microscopy (STM) (231), angle-resolved photoemission spectroscopy (ARPES) (253), and transport measurements (254) have contributed enormously to the characterization of the CDW/PLD phases in $1 T-\mathrm{TaS}_{2}$ and related TMDCs since the 1970s. However, many material features and couplings are only accessible in the time domain. With the advent of ultrashort laser pulses, it has become possible to drive specimens into transient or metastable states that do not occur in thermodynamic equilibrium - sometimes called hidden states. These transient states can be described as a function of photodoping density and time $(13,255)$. The combination of a pump/probe scheme with most of the aforementioned techniques has allowed for invaluable insights into the femtosecond coupling of the different degrees of freedom.

The hidden $(\mathrm{H})$ metallic CDW state in $1 T-\mathrm{TaS}_{2}$ is a popular example of how the high peak intensity of ultrashort laser pulses facilitates the discovery of metastable states. The $\mathrm{H}$ state can be reached by quenching the insulating C CDW using a single 35 fs laser pulse with a fluence of $\sim 1 \mathrm{~mJ} / \mathrm{cm}^{2}$ or a pulsed current. The specimen can be reverted to its ground state by applying a train of longer pulses, by Joule heating or by thermally heating the specimen above $70 \mathrm{~K}$ $(14,256-260)$. There are indications that the formation of the hidden state is connected to a collapse of interlayer dimers, i.e., to a change in CDW stacking order with respect to the stacking of the C CDW (261). In this section, however, we concentrate on photoinduced transitions between the thermodynamically stable CDW phases of $1 T-\mathrm{TaS}_{2}$ and possible intermediate states. For example, a transient $N C^{*}$ phase has been proposed which can be reached after intense photodoping of the $\mathrm{C}$ phase (255). Still, the most investigated intermediate is the gapless transient state that is reached after a slight optical perturbation of the $\mathrm{C}$ or NC phase $(239,241,242)$.

Before the advent of time-resolved ARPES (tr-ARPES) instrumentation, transient optical reflectivity measurements have been used to detect the subsequent relaxation of the carrier system (Fig. 3.9A). Right after the initial quench of the CDW order in the C or NC phase, two separate dynamic components are visible: an electronic single-particle response due to photoexcited charge carriers and an overlaid periodic perturbation of the charge density due to a coherent excitation of the CDW amplitude mode at a frequency of roughly $2.4 \mathrm{THz}$ at the lowest experimental temperature (262). 

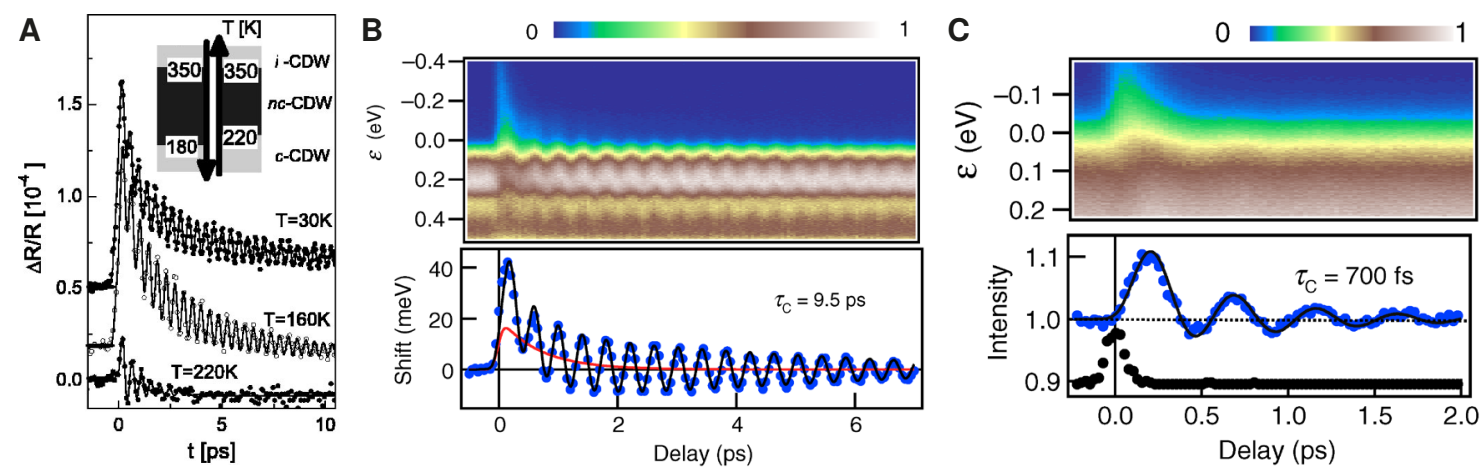

Figure 3.9: Electronic dynamics in $\mathbf{1 T}-\mathrm{TaS}_{\mathbf{2}}$ after an optical CDW quench. (A) Optical transient reflectivity curves at temperatures within the $\mathrm{C}$ phase (top and center) and the $\mathrm{NC}$ phase (bottom). The oscillatory response after weak optical excitation is caused by the coherent excitation of the CDW amplitude mode. The time constant of the damping and the mode frequency depend on the base temperature. Reprinted with permission from Ref. (262). Copyright 2002 by the American Physical Society. (B) The long-lasting oscillation of the amplitude mode in the $\mathrm{C}$ phase is also visible in tr-ARPES data measured near the $\Gamma$ point (top, $30 \mathrm{~K}$ base temperature, $135 \mu \mathrm{J} / \mathrm{cm}^{2}$ optical excitation). The electronic gap is filled by transient electronic states right after time-zero and recovers on a $680 \mathrm{fs}$ time scale (bottom, red line). The excitation of the amplitude mode leads to an additional periodic shift of the binding energies until damped after $9.5 \mathrm{ps}$ (blue dots and black line). (C) In tr-ARPES data measured in the NC phase (top, $300 \mathrm{~K}$ base temperature), the damping of the amplitude mode happens on a shorter $700 \mathrm{fs}$ time scale (bottom, blue dots and black line). The black dots indicate the temporal resolution. B and C reprinted from Ref. (242) with permission from the authors.

tr-ARPES has later been shown to yield information going beyond that obtained from transient reflectivity measurements $(239,241-244,263,264)$. For the first time, photoelectron spectra as a function of time allowed to disentangle the dynamics of the amplitude mode from a collapse of the electronic gap in the center of the Brillouin zone (Fig. 3.9B; see also the left panel of Fig. 3.7 for a momentum-resolved band structure of the $\mathrm{C}$ phase including lower-lying bands). Following a weak optical stimulus, transient electronic states emerge within the gap and coherent oscillations of the C CDW amplitude mode set in, evidencing strong electron-lattice coupling. The restoral of the electronic gap was found to follow a single-exponential behavior with a sub-picosecond time constant. Simultaneously, the oscillations of the amplitude mode cause a periodic shift of the photoemission spectra for a more extended period of time. Based on the decoupled nature of both dynamical features, it was concluded that the gap is of purely electronic nature and that the $\mathrm{C}$ phase of $1 T-\mathrm{TaS}_{2}$ is in fact Mott insulating $(241,242)$.

In case of the NC phase, transient reflectivity and tr-ARPES measurements (Fig. 3.9A and C) agree that the damping of the amplitude mode after quenching the NC order happens on a much shorter time scale. The weak damping in the $\mathrm{C}$ phase is attributed to the presence of the Mott gap such that the low-energy amplitudons can only scatter with other phonons on a several-picosecond time scale. Additionally, the lack of commensurability in the NC phase may 

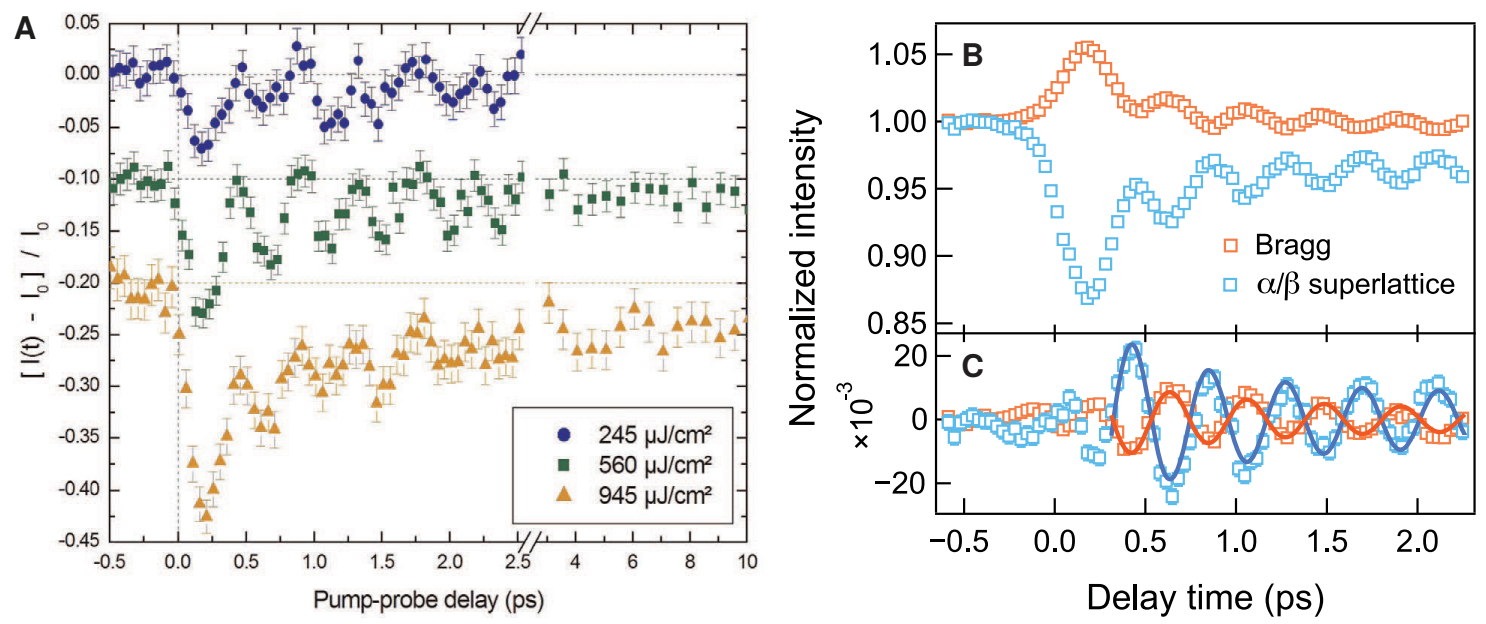

Figure 3.10: Structural dynamics in $1 T-\mathrm{TaS}_{2}$ after an optical CDW quench. (A) Periodic modulation of superstructure spot intensity in the $\mathrm{C}$ phase observed using ultrafast $\mathrm{x}$-ray diffraction ( $100 \mathrm{~K}$ base temperature). Reprinted from Ref. (256). Copyright 2014, with permission from Elsevier. (B) In the presence of the coherently oscillating amplitude mode, diffracted intensity is redistributed periodically between structural and superstructure reflections. Time traces acquired using MeV UED ( $40 \mathrm{~K}$ base temperature, $1 \mathrm{~mJ} / \mathrm{cm}^{2}$ optical excitation). (C) The intensity redistribution becomes even clearer after subtracting a single-exponential incoherent contribution from B. B and C reprinted from Ref. (265). Licensed under CC BY-NC 4.0.

lead to a broader spectrum of the amplitude mode, resulting in a faster dephasing of the coherent oscillations (242). More recent tr-ARPES approaches have succeeded in acquiring spectra in a larger portion of the Brillouin zone, specifically close to the $\Gamma$ point (Mott gap) and the $M$ point (CDW gap), by using femtosecond XUV instead of near-UV pulses $(239,263,264)$. Aided by improved temporal resolution, the experiments confirmed that the electronic Mott gap collapses on a sub-20 fs electron-hopping time scale, while the structural component acts as a bottleneck for the CDW melting process. The fastest pathway is a coherent relaxation of the amplitude mode which takes at least a half-cycle of the oscillation period $(\sim 200 \mathrm{fs})(239,244,264)$.

While transient reflectivity and tr-ARPES measurements access the electronic structure of a material, complementary structural insights can be obtained using diffraction techniques. ARPES spectra of the NC or IC phase are rare, whereas most ultrafast diffraction experiments have focused on the transition between those two phases $(65,222,250,266,267)$, with some exceptions in Refs. $(237,256,265)$. A coherent excitation of the amplitude mode in the $C$ phase of $1 T-\mathrm{TaS}_{2}$, while not affecting the symmetry of the material and the corresponding scattering vectors, periodically modulates the superstructure spot intensities (Fig. 3.10). More specifically, diffracted intensity is periodically redistributed between superstructure and structural Bragg spots - the closer the transient state is to the undisturbed metallic structure, the stronger the redistribution towards the structural reflections. 
A

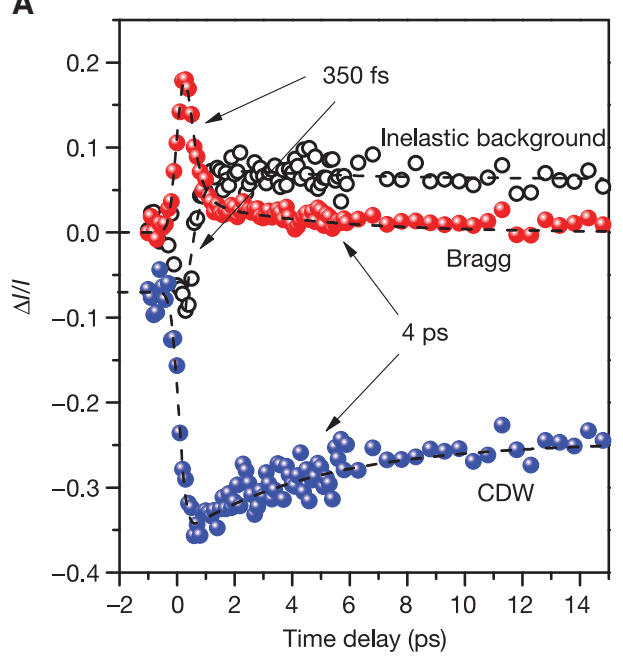

B

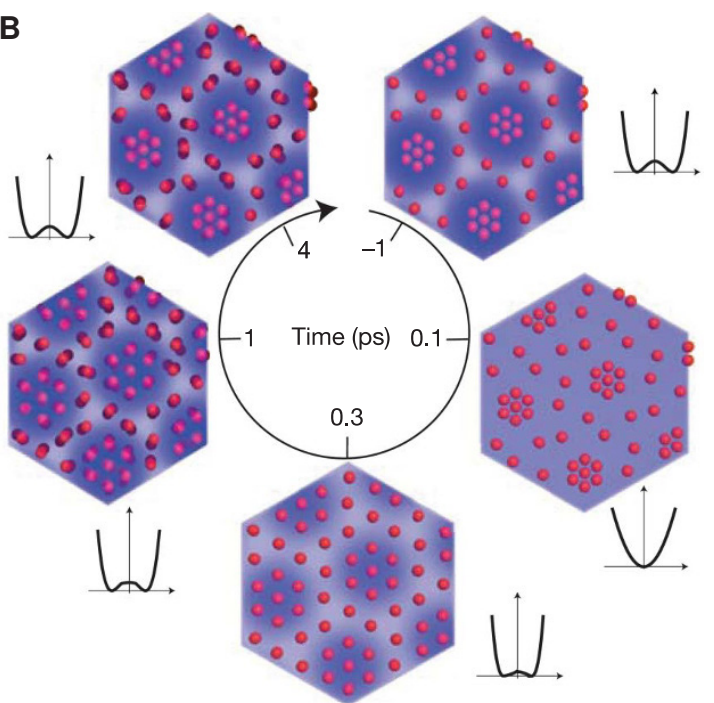

Figure 3.11: Ultrafast NC CDW/PLD quench in $1 T$-TaS ${ }_{2}$ observed by UED. (A) Ultrafast diffraction curves ( $200 \mathrm{~K}$ base temperature, $2.4 \mathrm{~mJ} / \mathrm{cm}^{2}$ optical excitation) averaged over all individual Bragg (red) and CDW (blue) reflections, and the inelastic background (open circles). The decrease in background intensity right after time-zero is an artifact due to tails of CDW peaks that reach into the region where the background intensity was evaluated. (B) Evolution of the real-space structure (red dots), the electron density (blue background color) and the underlying free-energy landscape as derived from UED and tr-ARPES data. Reprinted by permission from Springer Nature Customer Service Centre GmbH: Springer Nature, Nature, Ref. (65), Copyright 2010 Nature Publishing Group, a division of Macmillan Publishers Limited.

Similar behavior of intensity redistribution was first observed in the NC phase by EICHBERGER et al. in their pioneering UED work shown in Fig. 3.11 (65). Before time-zero, atomic structure and charge-density modulation are in thermal equilibrium, as shown by red dots and the modulated background color in panel B at -1 ps. The double-well shape of the underlying free-energy landscape indicates the thermodynamic stability of the NC phase. Right after time-zero, the perturbation of the electronic structure reshapes the potential towards an unmodulated state before the atomic structure is able to react (panel B at $0.1 \mathrm{ps}$ ). The rapid increase in structural intensity and the decrease in CDW intensity visible in panel $\mathrm{A}$ is interpreted in terms of a melting of the PLD along the coordinate of the amplitude mode (red dots in panel B at $0.3 \mathrm{ps}$ ). Using a fitting procedure, a time scale of 250(70) fs is extracted for this process (65) which is in agreement with tr-ARPES data $(239,244,264)$.

Parallel to the onset of the atomic motion, which is reflected by a relaxation of the structural order parameter towards the new potential minimum, the electron system starts to cool and restore the initial double-well shape of the potential. This cooling is mediated by scattering with strongly coupled phonon modes and leads to a redistribution of intensity from structural reflections into inelastic background on a $350 \mathrm{fs}$ time scale. The CDW order has partially recovered after $1 \mathrm{ps}$, 
and electron-lattice equilibration is understood to lead to a system that is thermalized at a higher temperature after $\sim 4 \mathrm{ps}$. The data in panel A, however, does not show oscillatory behavior although observed in an accompanying transient reflectivity measurement acquired under the same conditions. The authors attribute this to an oscillation amplitude that is buried within the noise level of the experiment (65). A direct observation of the amplitude mode is also more challenging in the $\mathrm{NC}$ than in the $\mathrm{C}$ phase due to the stronger damping.

While the general picture described in Fig. 3.11B is widely accepted in the literature, the precise pathways and time scales of thermalization are still a matter of contemporary debate. In a recent ultrafast low-energy electron diffraction (ULEED) experiment extending to $1500 \mathrm{ps}$ temporal delay, a slowdown of the relaxation dynamics in the NC and IC phase around 4 ps has indeed been confirmed. However, it was inferred from the intensity ratio of structural and satellite reflections that a second stage relaxation process extends until $60 \mathrm{ps}$ before reaching thermal equilibrium. For the case of the IC phase, this was explained by a decoupling of remaining excitations of the amplitude and phase modes from the electronic subsystem, and by the presence of topological defects (250).

Topological defects are known to play a crucial role in the phase formation kinetics after an optically induced NC/IC phase transition in $1 T-\mathrm{TaS}_{2}(222,265)$, as well as in other CDW materials $(223,268)$. Specifically, the CDW diffraction spots do not rotate during the phase transition - there is no global deformation that would transform the NC into the IC phase-but the IC modulation emerges after the suppression of the NC CDW, initially inhibiting longrange order $(222,266)$. An indicator for the presence and following annihilation of topological defects after the phase transition is a temporal narrowing of the IC diffraction spot width, i.e., the correlation length of the IC phase increases with time as observed using ULEED (222, 269). While this behavior had already been reported in UED and ultrafast x-ray diffraction experiments, it was previously explained in terms of a growth of the IC phase by a broadening of the discommensurations already present in the NC phase (266), and in terms of nucleation and growth of well-defined IC domains (267). The growth of the IC correlation length was found to follow a universal scaling law $\Delta t^{0.5}(222,267)$. Besides the observed narrowing of IC diffraction spots, an increase in IC spot intensity is observed over several hundred picoseconds, while the NC suppression shows the sharp threshold behavior described above $(222,266)$.

For the nanoimaging experiment presented in Chapter 5, two distinct regimes of photoinduced dynamics in $1 T-\mathrm{TaS}_{2}$ are relevant: A slight optical quench of the NC CDW order that is insufficient to drive the system into the IC phase [cf. Refs. $(65,250)]$, and a strong excitation that melts the NC phase and leads to the formation of a stable IC phase [cf. Refs. $(222,266,267)]$. 


\subsection{Kinematic diffraction simulation of the NC phase in $1 T-\mathrm{TaS}_{2}$}

The charge-density modulation in the NC phase of $1 T-\mathrm{TaS}_{2}$ is not purely sinusoidal as evidenced by its block-wave appearance. Higher harmonics of the modulation wave vectors $\vec{Q}_{i}$ play a decisive role in the formation of the commensurate domains separated by discommensurations (182). Accordingly, a Fourier series describing the atomic displacements $\vec{u}_{i}$ needs to be plugged into Eq. 3.5 for a more accurate result within the kinematic diffraction theory.

In their work, SpIJKerman et al. used 21 and 12 harmonics to describe the modulation of the tantalum and sulfur positions, respectively (182):

$$
\begin{aligned}
u_{\alpha}^{\mu}(\vec{r})=\sum_{n_{1}, n_{2}} A_{n_{1} n_{2} \alpha}^{\mu} \sin \left(2 \pi n_{1} \vec{Q}_{1} \cdot \vec{r}+2 \pi n_{2} \vec{Q}_{2} \cdot \vec{r}\right) \\
+B_{n_{1} n_{2} \alpha}^{\mu} \cos \left(2 \pi n_{1} \vec{Q}_{1} \cdot \vec{r}+2 \pi n_{2} \vec{Q}_{2} \cdot \vec{r}\right) .
\end{aligned}
$$

Here, $\alpha=1,2,3$ is the vector component and $\mu$ indicates the atom. The amplitudes $A_{n_{1}}^{\mu} n_{2} \alpha$ and $B_{n_{1} n_{2} \alpha}^{\mu}$ for all included harmonics $n_{1}$ and $n_{2}$ are given in Ref. (182) in units of $\AA$ along the normalized lattice vectors $\vec{a}_{\alpha} /\left|\vec{a}_{\alpha}\right|$. For tantalum, all $B_{n_{1} n_{2} \alpha}^{\mu}$ vanish due to the crystal site symmetry. The wave vectors $\vec{Q}_{1}=\left(\sigma_{1}, \sigma_{2}, \sigma_{3}\right)$ and $\vec{Q}_{2}=\left(-\sigma_{2}, \sigma_{1}+\sigma_{2},-\sigma_{3}\right)$ are given in fractional coordinates with $\sigma_{1}=0.2448(2), \sigma_{2}=0.0681$ (2) and $\sigma_{3}=1 / 3$. This modulation function now allows to numerically calculate structure factors for structural and CDW reflections.
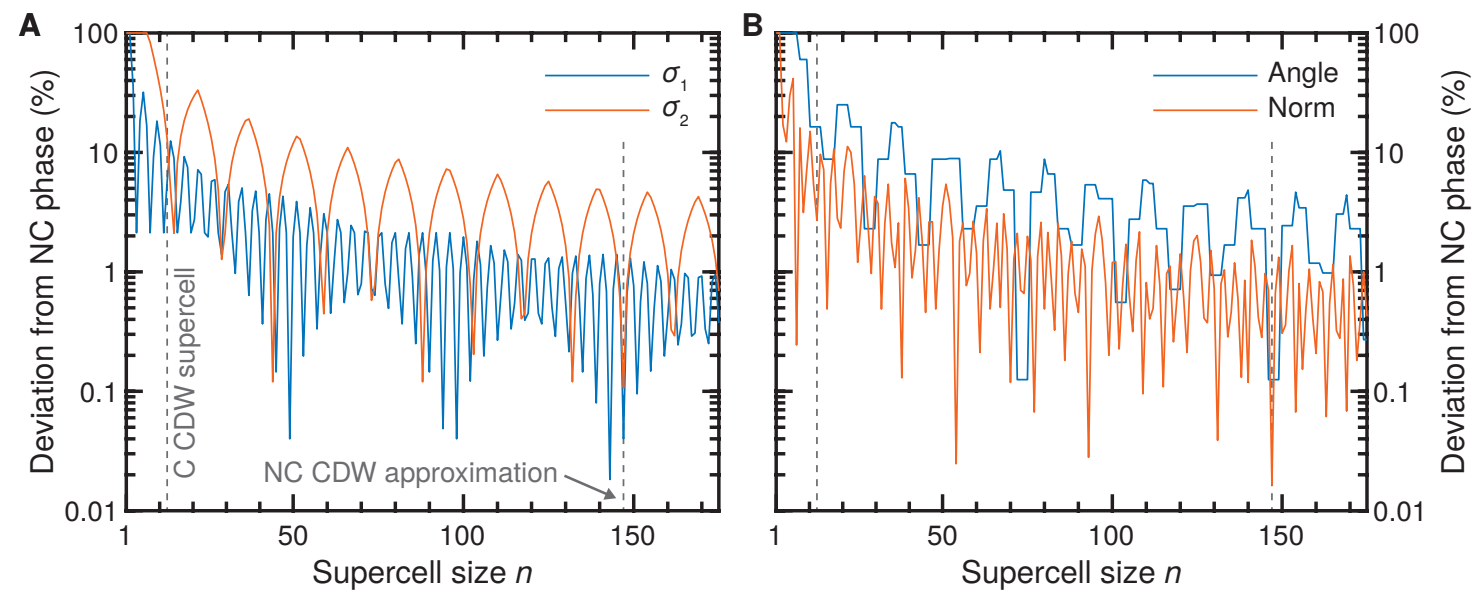

Figure 3.12: Supercell for "commensurate" NC CDW in 1T-TaS $\mathbf{S}_{2}$ (A) Relative deviations of $\sigma_{1}$ and $\sigma_{2}$ in a $n \times n$ commensurate supercell from the literature values in Ref. (182). The dashed line at $n=13$ indicates the supercell of the C CDW. A value of $n=147$ (second dashed line) is used in this work for the kinematic diffraction simulations of the NC CDW. (B) Relative deviations of the norm of the modulation wave vectors and of the angle with the structural lattice from literature values. 


\begin{tabular}{|l|ccccc|ccccc|}
\hline Atom species & $a_{1}$ & $a_{2}$ & $a_{3}$ & $a_{4}$ & $a_{5}$ & $b_{1}$ & $b_{2}$ & $b_{3}$ & $b_{4}$ & $b_{5}$ \\
\hline Sulfur & 0.2497 & 0.5628 & 1.3899 & 2.1865 & 0.7715 & 0.2681 & 1.6711 & 7.0267 & 19.5377 & 50.3888 \\
\hline Tantalum & 1.0190 & 2.2291 & 3.4097 & 3.9252 & 2.2679 & 0.2694 & 1.7962 & 7.6944 & 31.0942 & 91.1089 \\
\hline
\end{tabular}

Table 3.1: Coefficients for atomic scattering factors of sulfur and tantalum. Atomic scattering factors up to $\sin \theta / \lambda=2 / \AA$ can be calculated according to Eq. 2.5. Coefficients taken from Ref. (99).

In order to obtain translational symmetry, the in-plane NC CDW modulation wave vector components have been slightly modified to form a "commensurate" NC CDW in a supercell of $n \times n$ undistorted unit cells. The relative deviations of the resulting commensurate values for $\sigma_{1}$ and $\sigma_{2}$ from the literature values are shown in Fig. 3.12A as a function of $n$. At a value of $n=147$, both curves show a local minimum with $\sigma_{1}=36 / 147$ and $\sigma_{2}=10 / 147$. Higher values of $n$ would only increase the computational complexity without adequate benefits for the calculated structure factors. The norm and angle of the CDW wave vector with respect to the structural lattice amount to $0.9797 / \mathrm{nm}$ and $11.93^{\circ}$ at $n=147$, while SPIJKERMAN et al. report $0.9795 / \mathrm{nm}$ and $11.94^{\circ}$ (see Fig. 3.12B for the relative deviation of these values as a function of $n$ ). Atomic scattering factors for tantalum and sulfur are calculated according to Eq. 2.5 with the coefficients from Table 3.1. Based on the above approach, the kinematic diffraction simulations referenced in section 5.2.6 were carried out.

\subsection{Intercalated TMDCs}

In intercalated TMDCs, the intercalant atoms occupy well-defined octahedral or tetrahedral interstitial sites between the weakly bound layers as shown in Fig. 3.1E, forming the compound $\mathrm{A}_{y} \mathrm{MX}_{2}$ with the intercalant species $\mathrm{A}$. The process of intercalation is generally associated with a charge transfer from the intercalant species to the host, modifying the bond between trilayers towards an ionic bond (for the intercalation of alkali metals) or a covalent bond (for the intercalation of $3 d$ transition metals). Additionally, long-chain organic molecules are suitable for intercalation which can lead to a drastic increase in layer separation in $1 T-\mathrm{TaS}_{2}$ from the non-intercalated value of $5.90 \AA$ to up to $57 \AA(181,182)$.

In a first approximation, the charge transfer changes the filling of the transition metal $d$-bands which form the conduction band, and the topology of the Fermi surface whose characteristics becomes more three-dimensional. By introducing different intercalates into a known and wellstudied host system such as $\mathrm{TaS}_{2}$, the electronic and magnetic properties of the material can be fine-tuned in a unique way (181). This includes metal-semiconductor transitions (and vice versa) and the formation of ferro- and antiferromagnetic phases in the material $(181,270)$. 
As an example of an alkali intercalant species, lithium atoms can be introduced between the layers of $1 T$ and $2 H$ TMDCs, either electrochemically or by immersing the material in a suitable solution. In an electrochemical cell, a deintercalation process can be initiated by applying a reversed bias. The diffusion of lithium atoms between interstitial sites is a thermally activated process. In-plane long-range ordering of intercalant atoms is observed for specific intercalant concentrations and under suitable temperature and pressure conditions. Typical orderings are $2 \times 2$ and $\sqrt{3} \times \sqrt{3}$, corresponding to a concentration of $y=1 / 4$ and $1 / 3$, respectively. While an intercalant species may either occupy octahedral or tetrahedral interstitial sites, there are also more complex cases in which both sites are occupied (e.g., for silver intercalation) (181).

The process of lithium intercalation is of great technological importance because it is the basis for rechargeable lithium-ion batteries. Nowadays, these are in widespread use as the main power source of, for example, portable electronic devices and electric vehicles. In commercial lithium-ion batteries, a lithium deintercalation and intercalation process occurs simultaneously at a layered oxide cathode and a graphite anode (4). However, due to the relatively low specific capacity of graphite, new anode materials are needed to produce high-capacity lithium-ion batteries (271). TMDCs or heterostructures combining different two-dimensional materials may be possible candidates for higher-capacity anode materials $(30,272,273)$.

The ordering of intercalant atoms in such intercalation processes can be investigated, statically or even in situ, using electron imaging and diffraction (274). Ordered superlattices cause the appearance of superstructure reflections in diffraction images. A $2 \times 2$ ordering, for example, causes superstructure reflections halfway between the structural reflections of the host lattice, reflecting the doubled in-plane lattice constant.

More generally, the superstructure reflections of an $n \times n$ intercalant ordering appear at the same positions in reciprocal space at which the satellite reflections of an $n \times n \mathrm{C} \mathrm{CDW}$ would emerge. Apart from that, the physics governing the spot intensities corresponding to both phenomena is quite different. While the satellite spot intensities of a CDW, as outlined in section 3.2, are governed by an infinite sum of phase factors weighed by Bessel functions, the spot intensities of an intercalant superlattice are given by the finite sum over all phase contributions of the atoms in the $n \times n$ supercell (section 2.3). Interestingly, CDW formation and ordered intercalant superlattices can coexist in certain TMDCs such as $1 T-\mathrm{TaS}_{2}$ and $2 \mathrm{H}-\mathrm{TaSe}_{2}$. The intercalation of atoms or molecules in a CDW material can either modify or stabilize (275-278), or suppress the CDW ordering $(181,279-281)$. 


\subsection{Properties of $\mathrm{Mn}_{0.25} \mathrm{TaS}_{2}$ and $\mathrm{Fe}_{0.25} \mathrm{TaS}_{2}$}

The $3 d$ ion-intercalated TMDCs $\mathrm{Mn}_{0.25} \mathrm{TaS}_{2}$ and $\mathrm{Fe}_{0.25} \mathrm{TaS}_{2}$ are two examples of an intercalated $2 H$ polytype $(184,282)$. Due to the strong covalent character of the bond between tantalum and manganese or iron, respectively, the material loses its two-dimensional electronic character, effectively suppressing CDW formation (283). Additionally, intercalant diffusion between the trilayers is inhibited in this case such that manganese and iron intercalation can only occur during compound growth (181). This is typically done using chemical vapor transport techniques $(270,282)$. Theoretically, the $1 T-\mathrm{TaS}_{2}$ polytype also supports $3 d$ ion intercalation, but these intercalants prefer octahedral sites and would partially substitute tantalum atoms (181).

The resulting isostructural compounds $\mathrm{Mn}_{0.25} \mathrm{TaS}_{2}$ and $\mathrm{Fe}_{0.25} \mathrm{TaS}_{2}$ show a long-range $2 \times 2$ inplane intercalant ordering, as depicted in Fig. 3.1E. The intercalant atoms are also ordered in the out-of-plane direction, resulting in the formation of infinite $\mathrm{A}-\mathrm{Ta}-\mathrm{A}$ chains. Higher intercalant concentrations can also lead to a $\sqrt{3} \times \sqrt{3}$ in-plane ordering in both materials $(270,284-286)$. Due to the charge transfer to the tantalum $5 d$ band, the intercalant atoms in both structures are in $2+$ valence states $(270,282)$. The lattice parameters and other material properties are summarized in Table 3.2.

\begin{tabular}{|l|ccc|}
\hline Compound & $\mathbf{M n}_{\mathbf{0 . 2 5}} \mathbf{T a S}_{\mathbf{2}}$ & $\mathbf{F e}_{\mathbf{0 . 2 5}} \mathbf{T a S}_{\mathbf{2}}$ & References \\
\hline Lattice constant $a(\AA)$ & $6.645(1)$ & $6.6141(15)$ & $(184,282)$ \\
\hline Lattice constant $c(\AA)$ & $12.552(2)$ & $12.154(3)$ & $(184,282)$ \\
\hline Intercalant valence & $\begin{array}{c}\mathrm{Mn}^{2+} \\
\left(d^{5} \text { configuration }\right)\end{array}$ & $\begin{array}{c}\mathrm{Fe}^{2+} \\
\left(d^{6} \text { configuration }\right)\end{array}$ & $(270,282)$ \\
\hline Curie temperature $T_{\mathrm{C}}(\mathrm{K})$ & 80 & 155 & $(270,287)$ \\
\hline Easy axis of magnetization $M$ & $M \perp c$ & $M \| c$ & $(282,288)$ \\
\hline
\end{tabular}

Table 3.2: Properties of $\mathbf{M n}_{0.25} \mathrm{TaS}_{2}$ and $\mathrm{Fe}_{0.25} \mathrm{TaS}_{2}$. The isostructural compounds crystallize in the $P 6_{3} / m m c$ space group. See Fig. 3.1E for a drawing of the crystal structure.

The literature reports a structural phase transition occurring in one of the two compounds, $\mathrm{Mn}_{0.25} \mathrm{TaS}_{2}$. The distance between manganese and tantalum in the chains along the $c$-axis is fixed by symmetry to be exactly $c / 4$. A variation in the $\mathrm{Ta}-\mathrm{Mn}$ bond distance therefore alters the length of the crystal's unit cell in a cooperative process. At a temperature of around $690 \mathrm{~K}$, the character of the manganese $3 d$ electrons transforms from a partly delocalized behavior at lower temperatures to a more localized behavior at higher temperatures, inducing a change of $1 \%$ in the ratio $c / a$ of the lattice constants $(184,284)$. The $\mathrm{Fe}_{0.25} \mathrm{TaS}_{2}$ system has been much less studied and, although sharing the same structure, there are no reports of a similar discontinuity. 
However, the magnetic properties of both compounds $\mathrm{Mn}_{0.25} \mathrm{TaS}_{2}$ and $\mathrm{Fe}_{0.25} \mathrm{TaS}_{2}$ are of primary relevance for the present thesis. Below the Curie temperatures of $80 \mathrm{~K}(270)$ and $155 \mathrm{~K}$ (287), respectively, ferromagnetic order emerges. Both materials show differences in the direction of magnetization, in coercivity and in the mechanisms leading to the ferromagnetic behavior. These details are outlined in section 4.2 of the first publication contributing to this cumulative thesis. While the static magnetic properties of these materials have been well-investigated in previous decades, there is a lack of time-resolved investigations. As demonstrated in Ref. (289), transient reflectivity and ultrafast magneto-optical spectroscopy measurements yield relevant information about the ultrafast demagnetization dynamics and the coupling of spin and lattice degrees of freedom.

In comparison to pristine or alkali-intercalated TMDCs, thin films of $3 d$ ion-intercalated TMDCs are difficult to obtain by exfoliation or cleavage because of the increased bonding between the layers (270). Many material investigations can be conducted using bulk materials. This includes optical spectroscopy and electron diffraction in reflection geometries, or X-ray magnetic circular dichroism (XMCD) studies in total electron yield (TEY) or fluorescence yield (FY) modes. All of these techniques can either be realized statically or time-resolved. Nevertheless, having access to electron- and photon-transparent thin films as reported in Chapter 4 enables the use of transmission geometries, e.g., for UTEM (Chapter 5) or ultrafast XMCD (290). 



\section{Chapter 4}

\section{Structural and magnetic characterization of large area, free-standing thin films of magnetic ion intercalated dichalcogenides $\mathrm{Mn}_{0.25} \mathrm{TaS}_{2}$ and $\mathrm{Fe}_{0.25} \mathrm{TaS}_{2}$}

Th. Danz, Q. Liu, X. D. Zhu, L. H. Wang, S. W. Cheong, I. Radu, C. Ropers, R. I. Tobey

J. Phys. Condens. Matter 28, 356002 (2016)

doi:10.1088/0953-8984/28/35/356002

Free-standing thin films of magnetic ion intercalated transition metal dichalcogenides are produced using ultramicrotoming techniques. Films of thicknesses ranging from $30 \mathrm{~nm}$ to $250 \mathrm{~nm}$ were achieved and characterized using transmission electron diffraction and $\mathrm{X}$-ray magnetic circular dichroism. Diffraction measurements visualize the long range crystallographic ordering of the intercalated ions, while the dichroism measurements directly assess the orbital contributions to the total magnetic moment. We thus verify the unquenched orbital moment in $\mathrm{Fe}_{0.25} \mathrm{TaS}_{2}$ and measure the fully quenched orbital contribution in $\mathrm{Mn}_{0.25} \mathrm{TaS}_{2}$. Such films can be used in a wide variety of ultrafast $\mathrm{X}$-ray and electron techniques that benefit from transmission geometries, and allow measurements of ultrafast structural, electronic, and magnetization dynamics in space and time.

This is the Accepted Manuscript version of an article accepted for publication in Journal of Physics: Condensed Matter. IOP Publishing Ltd is not responsible for any errors or omissions in this version of the manuscript or any version derived from it. The Version of Record is available online at doi:10.1088/0953-8984/28/35/356002. 


\subsection{Introduction}

Transition metal dichalcogenides (TMDCs) are a broad class of layered materials with a variety of structural and electronic properties. Depending on the specific compound and layer stacking, TMDCs can exhibit metallic (e.g. $\mathrm{TaS}_{2}$ ), semi-metallic (e.g. $\mathrm{WTe}_{2}$ ), semiconducting (e.g. $\mathrm{MoSe}_{2}$ ), or insulating (e.g. $\mathrm{HfS}_{2}$ ) behaviour $(5,6)$. Recent work has focused on the emergence and dynamics of charge density wave formation (commensurate and incommensurate) concomitant with metal-to-insulator transitions $(264,291)$. Yet other work dealt with the properties of monolayers of these materials. Loose interlayer bonding via van der Waals forces allows the fabrication of single monolayers of material which often exhibit electronic phases that are starkly different from bulk properties. Studies on monolayers have brought forth possibilities in optoelectronics and valleytronics (192, 292), and spintronics (193).

The intrinsic properties of the bulk materials can be further expanded by intercalation of atoms and small molecules between the layers. In particular, the incorporation of $3 d$ transition metals results in the onset of an array of magnetic properties which can be tuned by intercalation concentration, choice of intercalated species, and choice of host lattice (270). In total, the range of magnetic properties is enormous, providing a platform on which to study magnetism and domain structure as well as their dynamics.

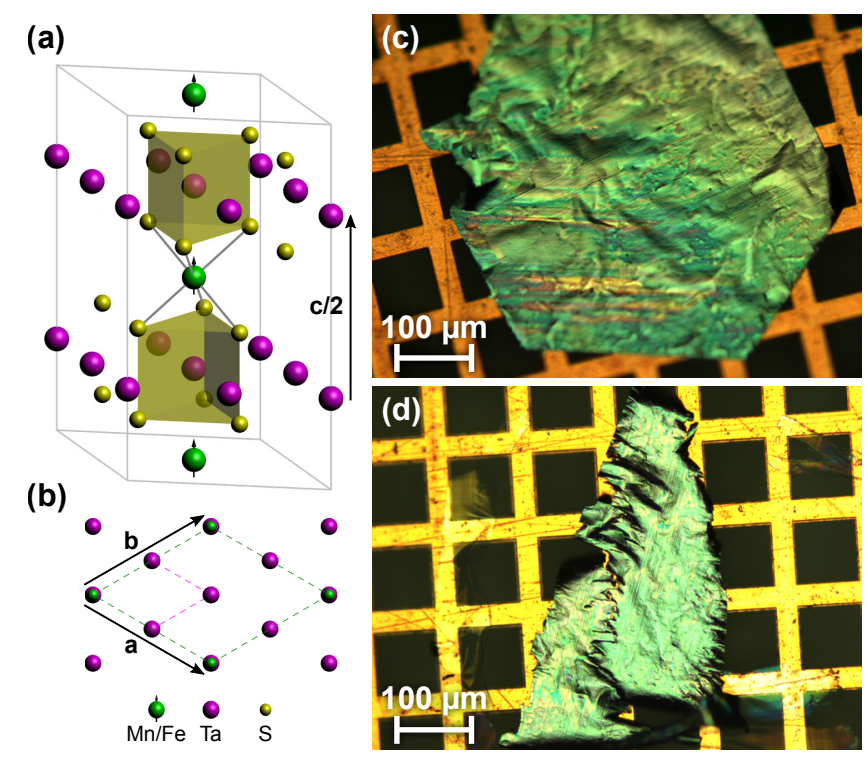

Figure 4.1: (a) Unit cell of $3 d$ ion intercalated TMDC samples. Trilayers of tantalum and sulfur are stacked along the $c$-axis. Magnetic ions fit between the layers in specific locations determined by the concentration $y$. (b) Planar view shows the crystallization of intercalants at $y=0.25$. (c) $100 \mathrm{~nm}$ sample of $\mathrm{Mn}_{0.25} \mathrm{TaS}_{2}$, and (d) $200 \mathrm{~nm}$ sample of $\mathrm{Fe}_{0.25} \mathrm{TaS}_{2}$ on a 200 lines per inch copper grid. 
Here we discuss a sample preparation technique that provides free-standing thin films of magnetic ion intercalated $\mathrm{TaS}_{2}$. In thin film form, these samples open the possibility for a number of magnetization dynamics studies with new techniques such as ultrafast electron diffraction $(65,293)$ and microscopy (176), as well as X-ray absorption (294), all of which benefit from transmission geometries where the penetration depth of both pump and probe can be easily matched. Moreover, for X-ray absorption studies, the transmission geometry is the most robust when compared to its total electron yield $\left(\mathrm{TEY}^{6}\right)$ or fluorescence yield (FY) counterparts in that it provides a direct measure of dichroic absorption and is unaffected by electronic saturation effects (298). The samples were prepared using ultramicrotoming techniques (106), and their structural and magnetic properties were measured using transmission electron diffraction and Xray circular magnetic dichroism, respectively. Two different materials were studied, $\mathrm{Mn}_{0.25} \mathrm{TaS}_{2}$ and $\mathrm{Fe}_{0.25} \mathrm{TaS}_{2}$, which differ in their magnetic properties while sharing the same structure.

\subsection{Material description}

TMDCs (chemical formula $\mathrm{MX}_{2}$ where $\mathrm{X}$ is a chalcogen and $\mathrm{M}$ a transition metal) consist of stacks of $\mathrm{X}-\mathrm{M}-\mathrm{X}$ sandwich layers which are van der Waals bonded along the $c$-axis (179). Variations in layer stacking result in a wide range of physical properties, while particular stacking configurations promote intercalation. For $\mathrm{TaS}_{2}$, the metallic $2 \mathrm{H}$ polytype facilitates intercalation. Upon intercalation, the new chemical formula is $\mathrm{A}_{y} \mathrm{MX}_{2}$, which in the context of TMDCs is understood to maintain the $\mathrm{MX}_{2}$ structure, while new ions are incorporated between the host layers. The process of $3 d$ ion intercalation occurs during the growth phase, for example using iodine vapor transport. Interestingly, particular intercalant concentrations lead to long-range crystalline order of intercalants between the host layers. This is the case for two situations, that of intercalant density $y=1 / 4$ and $y=1 / 3$, where the intercalants form an ordered $2 \times 2$ or $\sqrt{3} \times \sqrt{3}$ superlattice, respectively $(270,284,285)$. When such ordered states occur, the intercalated ion is known to reside in specific sites of the host lattice (the trigonal antiprismatic hollow site between the layers of $2 \mathrm{H}-\mathrm{TaS}_{2}$ ), ensuring that the local environment of the magnetic ion is unchanged as one varies the intercalant species or host lattice. A three-dimensional view of the crystallographic structure is shown in Fig. 4.1(a) with specific emphasis on the location of the intercalant ion between $\mathrm{X}-\mathrm{M}-\mathrm{X}$ sheets. The crystallization of intercalants is best visualized in a planar view along the $c$-axis, as shown in Fig. 4.1(b).

\footnotetext{
${ }^{6}$ The original publication uses the incorrect abbreviation TIY at this point. A total ion yield measurement uses ionic currents to conduct X-ray absorption spectroscopy (XAS), e.g., in liquids and gases $(295,296)$. This is in analogy to a TEY measurement using electric currents when acquiring bulk XAS spectra (297).
} 
Bulk materials are characterized by Curie temperatures of $80 \mathrm{~K}$ for $\mathrm{Mn}_{0.25} \mathrm{TaS}_{2}$ (270) and $155 \mathrm{~K}$ (287) for $\mathrm{Fe}_{0.25} \mathrm{TaS}_{2}$. The large intercalant-intercalant distance inhibits direct exchange mechanisms, and an indirect RKKY interaction is understood to play a crucial role $(270,286)$. In this case, the exchange interaction between local moments is mediated by the conduction electrons of the host lattice. The isolated ions thus exhibit structural aspects reminiscent of local-moment magnetic systems. At the same time, there are indications of itinerant magnetism in $\mathrm{Mn}_{0.25} \mathrm{TaS}_{2}$ due to a strong hybridisation between the intercalant's $3 d$ states with the Ta $5 d_{z^{2}}$ states which form the conduction band (299). This class of materials thus bridges the divide between truly local-moment magnetism (as in $4 f$ systems) and itinerant ferromagnetism observed in $3 d$ transition metals.

The two materials are further distinguished by their magnetic orientation. While $\mathrm{Mn}_{0.25} \operatorname{TaS}_{2}$ is an easy plane ferromagnet with low coercivity (288), $\mathrm{Fe}_{0.25} \mathrm{TaS}_{2}$ exhibits a strong out-of-plane magnetic anisotropy with high coercivity (282). Additionally, as we will confirm, $\mathrm{Fe}_{0.25} \mathrm{TaS}_{2}$ is known to have a large orbital contribution to the magnetization, while the orbital contribution in $\mathrm{Mn}_{0.25} \mathrm{TaS}_{2}$ is fully quenched.

\subsection{Preparation of free-standing thin films}

TMDCs can be readily thinned down to monolayer thickness using the 'scotch tape method' (5), as well as by lithium intercalation (300) or by ultrasonification (301). However, due to charge transfer from the intercalant to the host and a concomitant change of the interlayer bonding towards a covalent bond (181), standard cleaving methods must be abandoned in the case of $3 d$ ion intercalated TMDCs. To our knowledge, there have been no reports of exfoliation of thin samples of $\mathrm{Mn}_{0.25} \mathrm{TaS}_{2}$ and $\mathrm{Fe}_{0.25} \mathrm{TaS}_{2}$.

Recently, the preparation of free-standing films of $1 T-\mathrm{TaS}_{2}$ using an ultramicrotome was reported (302). The resulting films had lateral dimensions of $\sim 200 \mu \mathrm{m}$ and thickness of $30 \mathrm{~nm}$. On the basis of supporting transmission electron microscope (TEM) diffraction images, it has been shown that the crystallinity of the sample and the transition temperatures between the different charge density wave phases are unaffected, and that the preparation principle is suitable for making thin TMDC samples. Following this demonstration, we applied similar methodologies, however here to the more strongly bonded $3 d$ ion intercalated TMDCs.

Following standard techniques used in specimen sectioning (106), we embedded $\mathrm{Mn}_{0.25} \mathrm{TaS}_{2}$ and $\mathrm{Fe}_{0.25} \mathrm{TaS}_{2}$ bulk crystals with lateral dimensions of $\sim 500 \mu \mathrm{m}$ in transparent epoxy (Epofix Cold-Setting Embedding Resin) for cutting in a Leica EM UC6 ultramicrotome. After mixing 
resin and hardener, the epoxy was heated for a few minutes in an oven at $60{ }^{\circ} \mathrm{C}$ in order to lower its viscosity and remove air bubbles. Afterwards, we covered the bottom of $15 \mathrm{~mm} \times 7 \mathrm{~mm}$-sized molds with a thin epoxy film and cured the epoxy for $\sim 30 \mathrm{~min}$ at $60^{\circ} \mathrm{C}$. Then we cut scratches in the epoxy layers using a razor blade, introduced bulk crystals in an upright position and filled the rest of the molds with epoxy. After another $\sim 3 \mathrm{~h}$ of curing at $60^{\circ} \mathrm{C}$, the epoxy blocks were ready for sectioning. Epoxy blocks were trimmed to expose the $a b$-plane of the sample material. After pre-sectioning with a glass knife, a diamond knife (dEYEmond ULTRA $45^{\circ}$ ) was used to produce thin sections from the block face. These were transferred onto square mesh copper TEM grids using a metallic loop suspending the sections in a thin water film ('perfect loop'). As read out from the ultramicrotome settings, we were able to obtain large area sections from $250 \mathrm{~nm}$ down to $30 \mathrm{~nm}$ in thickness. We found that $\mathrm{Mn}_{0.25} \mathrm{TaS}_{2}$ was more easily sectioned than $\mathrm{Fe}_{0.25} \mathrm{TaS}_{2}$, which had a tendency to disintegrate during cutting. We attribute this to deformations of the $\mathrm{Fe}_{0.25} \mathrm{TaS}_{2}$ lattice induced during the clipping of a small fraction from a spatially more extended crystal platelet. For $\mathrm{Mn}_{0.25} \mathrm{TaS}_{2}$ in contrast, we had suitably sized bulk crystals available from the start.

While sample thicknesses were as-read from the microtome settings, the $\mathrm{Mn}_{0.25} \mathrm{TaS}_{2}$ sample displayed in Fig. 4.1(c) was also tested via electron energy loss spectroscopy (EELS) to assess the thickness (109). Assuming an effective atomic number of $Z=34$, thickness measurements of three locations of the sample resulted in values ranging from $80 \mathrm{~nm}$ to $100 \mathrm{~nm}$ (with $\pm 20 \%$ accuracy), in close agreement with the $100 \mathrm{~nm}$ as-read value. Furthermore, all measurements relating to structural (electron diffraction) and magnetic (X-ray dichroism) properties remain qualitatively similar regardless of thickness.

\subsection{Characterization}

\subsubsection{Optical microscopy}

Optical microscopy was conducted for every sample, representative images of which are shown in Fig. 4.1(c) and (d). The sectioned samples showed large lateral areas of several hundreds of microns for $\mathrm{Mn}_{0.25} \mathrm{TaS}_{2}$ and slightly smaller for $\mathrm{Fe}_{0.25} \mathrm{TaS}_{2}$, and a good adhesion to the grids.

In the optical images, we can identify regions of the samples that appear grooved. This is most easily seen as horizontal lines near the bottom of Fig. 4.1(c). The grooves correspond to the cutting direction of the ultramicrotome and indicate imperfections that develop during multiple uses of the diamond knife. In Ref. (302), it is suggested that this is caused by a reaction 
of the tantalum with the diamond blade. In our studies, we did not use a virgin knife, and thus we are unable to classify the origin of the knife damage. However, over the course of many cuts, we note that the density of scratches and nicks in the blade did not noticeably increase, while the sample quality remained unchanged.

Despite the structural inhomogeneities that are caused by our preparation technique, we presently do not witness modifications to the sample magnetic properties, for example due to cut-induced strain. In particular, in the X-ray studies presented below, the onset of magnetic properties occurs at the same temperature (within $10 \mathrm{~K}$ ) of the pre-cut bulk samples (measured using MPMS). We take this as an indication of limited strain-induced modification to the magnetic properties.

\subsubsection{Transmission electron diffraction}

In order to verify the crystallinity of the sectioned samples, and particularly to investigate the ordering of intercalated ions, we recorded selected area electron diffraction (SAED) patterns in a TEM. Images recorded at $200 \mathrm{keV}$ electron energy are shown in Fig. 4.2. For $\mathrm{Fe}_{0.25} \mathrm{TaS}_{2}$, a thinner edge region was required in order to clearly witness a nice diffraction pattern. Importantly, both diffraction patterns exhibit the expected superlattice reflections midway between the structural reflections of the host lattice, indicative of the $2 \times 2$ ordering of the intercalants. Furthermore, we observe no sign of a $\sqrt{3} \times \sqrt{3}$ superlattice which would emerge in case of intercalant excess or disorder (303). In general, good quality diffraction patterns were acquired on the thinnest samples (XMCD measurements discussed below can be performed on all sample thicknesses), while $\mathrm{Mn}_{0.25} \mathrm{TaS}_{2}$ showed better spatial homogeneity in intercalant concentration than $\mathrm{Fe}_{0.25} \mathrm{TaS}_{2}$.
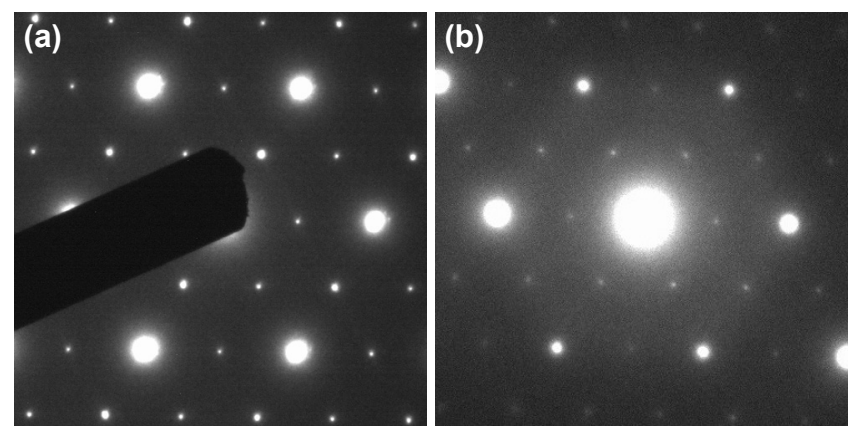

Figure 4.2: Selected area electron diffraction (SAED) images of (a) $50 \mathrm{~nm}$ sample of $\mathrm{Mn}_{0.25} \mathrm{TaS}_{2}$, and (b) $200 \mathrm{~nm}$ sample of $\mathrm{Fe}_{0.25} \mathrm{TaS}_{2}$. Weak superlattice spots are visible in both diffraction patterns halfway between the structural reflections of $2 \mathrm{H}-\mathrm{TaS}_{2}$. The images are scaled to accommodate different camera lengths. 


\subsubsection{XMCD spectroscopy}

To assess the magnetic properties of the thinned samples, X-ray magnetic circular dichroism (XMCD) measurements in a transmission geometry were performed using the ALICE chamber at beamline PM3 of the BESSY II synchrotron at Helmholtz-Zentrum Berlin. The ALICE chamber features a liquid helium cooled sample holder and an electromagnet, both of which rotate with respect to the incoming beam direction and facilitate the study of both out-of-plane and in-plane magnetization characteristics. Details on the broader versatility of ALICE can be found in Ref. (297). Measurements were conducted at a range of temperatures below the respective Curie temperatures of the two samples. Full spectroscopic information over the $L_{3,2}$ absorption edges of Fe and Mn was collected. Since XMCD is sensitive to the magnetization component parallel to the X-ray wave vector, measurements of $\mathrm{Fe}_{0.25} \mathrm{TaS}_{2}$ were performed at normal incidence, while $\mathrm{Mn}_{0.25} \mathrm{TaS}_{2}$ (and the applied field) was rotated by an angle $\Theta$ with respect to the X-ray beam direction to maximize the projection of the magnetization onto the $\mathrm{X}$-ray wave vector. Rotation angles achieved did not exceed $40^{\circ}$ from normal. For a fixed X-ray helicity, the direction of the magnetic field was switched, and the transmitted intensity was acquired for oppositely magnetized films, denoted as $I_{+}$and $I_{-}$, from which we calculate the absorption cross sections $\mu_{ \pm}=-\log \left(I_{ \pm} / I_{0}\right)$ for the two magnetization directions $\left(I_{0}\right.$ is the incident photon flux).

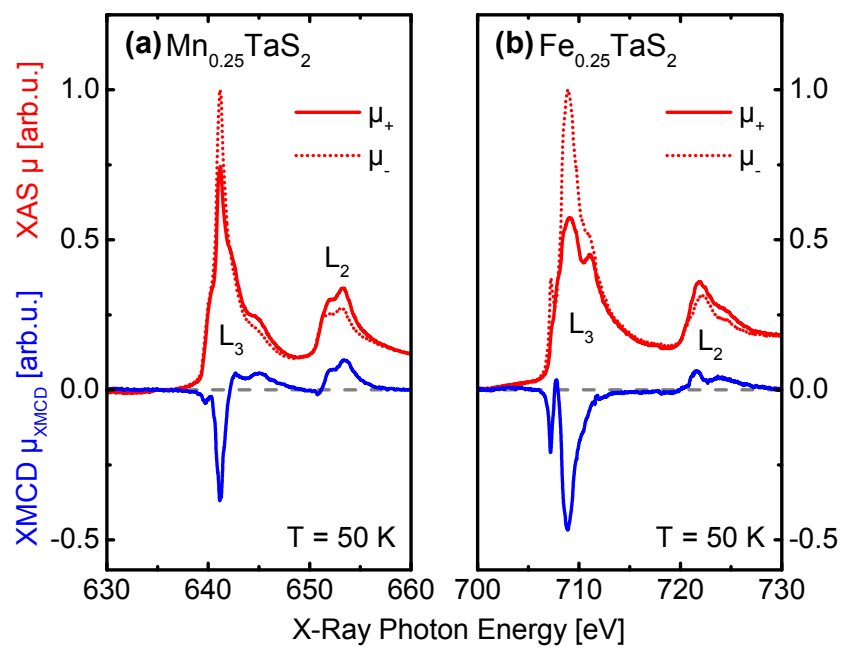

Figure 4.3: X-ray absorption spectra $\mu_{ \pm}$(red) for (a) $150 \mathrm{~nm}$ sample of $\mathrm{Mn}_{0.25} \mathrm{TaS}_{2}$, and (b) $200 \mathrm{~nm}$ sample of $\mathrm{Fe}_{0.25} \mathrm{TaS}_{2}$, for two orientations of applied magnetic field. The sample and applied field were rotated $40^{\circ}$ with respect to the incoming beam for $\mathrm{Mn}_{0.25} \mathrm{TaS}_{2}$, while $\mathrm{Fe}_{0.25} \mathrm{TaS}_{2}$ was acquired at normal incidence. XMCD spectra are shown in blue. The asymmetry in $L_{3}$ and $L_{2}$ for the case of $\mathrm{Fe}_{0.25} \mathrm{TaS}_{2}$ indicates a large unquenched orbital moment. 
Figure 4.3 shows representative X-ray absorption spectra $\mu_{ \pm}$(red) for $\mathrm{Mn}_{0.25} \operatorname{TaS}_{2}$ and $\mathrm{Fe}_{0.25} \mathrm{TaS}_{2}$ across the respective $L_{3,2}$ edges for both orientations of the magnetic field. Included in the figure is the extracted XMCD signal $\mu_{\text {ХмсD }}$ (blue) for the two materials, calculated following standard treatments for XMCD analysis $(304,305)$ :

$$
\mu_{\mathrm{xMCD}}(E)=\frac{1}{\gamma \cos (\Theta)}\left[\mu_{+}(E)-\mu_{-}(E)\right]
$$

The XMCD signal has been corrected for both angle of incidence $\Theta$ and degree of helicity of the source $\gamma$. We used $\gamma \approx .925$ which is characteristic for the off-axis emission of the bend magnet radiation at beamline PM3 (specifically at the Fe $L$ edge) (306).

A cursory comparison between the XMCD spectra for the two materials unveils a notable difference, namely, the integrated intensity of the $L_{3,2}$ edges are vastly different. As is well known from XMCD analysis, differences in the integrated intensity at the two edges is a manifestation of varying degrees of spin and orbital contributions to the total magnetization.

Applying sum rules to the respective edges, we can extract the ratio of orbital to spin magnetic moment (305):

$$
\frac{m_{\text {orb }}}{m_{\text {spin }}}=\frac{2 q}{9 p-6 q}
$$

where $p$ represents the area under the $L_{3}$ edge, and $q$ the overall area under both absorption edges:

$$
\begin{aligned}
& p=\int_{L_{3}} \mu_{\text {ХмСD }}(E) \mathrm{d} E \\
& q=\int_{L_{3}+L_{2}} \mu_{\text {ХмСD }}(E) \mathrm{d} E
\end{aligned}
$$

The measured ratio of orbital to spin magnetic moment for $\mathrm{Fe}_{0.25} \mathrm{TaS}_{2}$ is found to be .39 (4) and in close agreement with previously obtained values of .33 (286). Meanwhile the measured value for $\mathrm{Mn}_{0.25} \mathrm{TaS}_{2}$ is $-.06(6)$, which to our knowledge is the first direct measurement of the fully quenched orbital moment in this material. Previously, the existence of a quenched orbital moment was inferred by the isotropic magnetic susceptibility in the paramagnetic phase (270). The results and corresponding error bars are derived from the ensemble of measurements below $50 \mathrm{~K}$ and $110 \mathrm{~K}$ for $\mathrm{Mn}_{0.25} \mathrm{TaS}_{2}$ and $\mathrm{Fe}_{0.25} \mathrm{TaS}_{2}$, respectively.

As a final measurement, hysteresis curves were obtained at the peak of the $L_{3}$ edge for both samples, as shown in Fig. 4.4. In the case of $\mathrm{Mn}_{0.25} \mathrm{TaS}_{2}$, the hysteresis vanishes above $90 \mathrm{~K}$, in 


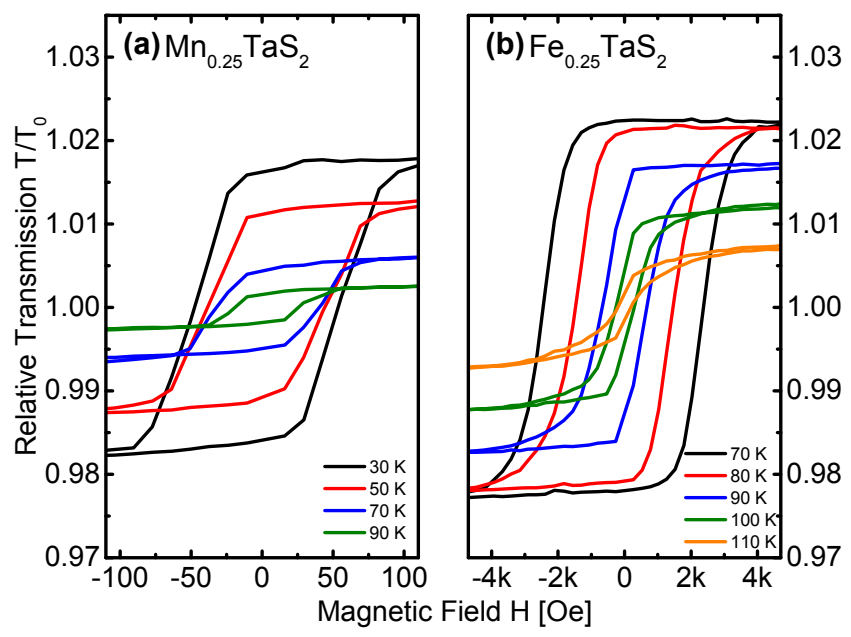

Figure 4.4: Hysteresis curves at the $L_{3}$ edge of (a) $50 \mathrm{~nm}$ sample of $\mathrm{Mn}_{0.25} \mathrm{TaS}_{2}$, and (b) $200 \mathrm{~nm}$ sample of $\mathrm{Fe}_{0.25} \mathrm{TaS}_{2}$. Note the different field strengths for the two samples.

close agreement with our independent measurements on the bulk samples prior to sectioning $\left(T_{\mathrm{C}}=96 \mathrm{~K}\right)$ using a magnetic property measurement system (MPMS). This lies $10 \mathrm{~K}$ above the literature value of $80 \mathrm{~K}$ (270) mentioned at the outset and indicates good sample quality, since off-stoichiometry drastically reduces the ferromagnetic transition temperature in $3 d$ ion intercalated TMDCs (307). For $\mathrm{Fe}_{0.25} \mathrm{TaS}_{2}$, we measure a Curie temperature of $\sim 110 \mathrm{~K}$, which agrees reasonably well with our independent MPMS measurements on the bulk samples (in zero-field-cooled measurements, we witness a broad transition region extending from $160 \mathrm{~K}$ to $120 \mathrm{~K}$ ). However, the measured transition temperature lies below the literature value of $155 \mathrm{~K}$ (287) for phase pure $\mathrm{Fe}_{0.25} \mathrm{TaS}_{2}$, and is likely indicative of regions of off-stoichiometry (307). This is also corroborated by our SAED results which indicate a degree of spatial inhomogeneity. Nonetheless, the expected high coercivity of the iron intercalated sample as compared to the manganese intercalated sample is clearly visible.

\subsection{Conclusion and outlook}

In conclusion, we prepared free-standing thin films of magnetic ion intercalated transition metal dichalcogenides using ultramicrotoming techniques. Thicknesses ranging between $30 \mathrm{~nm}$ and $250 \mathrm{~nm}$ were achieved and characterized by transmission electron diffraction and XMCD spectroscopy. Using transmission electron diffraction, we witness the intercalant $2 \times 2$ crystallographic ordering in both materials, a characteristic of the intercalation concentration $y=1 / 4$. Using XMCD spectroscopy, we measured the orbital contribution to the total magnetization for the two 
materials. Thin films facilitate true absorption measurements, and are preferred for quantitative measurements over fluorescence and electron yield geometries. This has allowed us to verify the unquenched orbital moment in $\mathrm{Fe}_{0.25} \mathrm{TaS}_{2}$, as well as the fully quenched orbital moment in $\mathrm{Mn}_{0.25} \mathrm{TaS}_{2}$.

Looking ahead, we note that thin films of intercalated dichalcogenides have wide applicability in time-resolved X-ray and electron diffraction studies. In both cases, the sample thickness can be optimized to easily match the absorption depth of both pump and probe pulses, ensuring that measurements are performed on uniformly excited materials. With respect to time-resolved $\mathrm{XMCD}(308,309)$, we foresee the capability of measuring magnetization dynamics of 'local moment' orbital and spin components on the intercalant site, as well as dynamics of itinerant conduction electrons of the host lattice (measured at a suitable host absorption edge). Due to the RKKY interaction, conduction electrons acquire a modest spin polarization which we expect exhibits different magnetization dynamics. In the broader context of intercalated dichalcogenides, as one measures dynamics in a variety of members of this family, the orbital, spin, and conduction electron contribution to the total magnetization can be varied, providing a robust platform on which to study ultrafast magnetism.

Finally, we also envisage a range of time-resolved coherent X-ray scattering measurements to resolve the dynamics of magnetic domains on sub-picosecond timescales, and length scales of a few tens of nanometers. For the case of ultrafast transmission electron microscopy (UTEM) $(130,147)$, thin magnetic films open the door to ultrafast Lorentz microscopy, thus providing a measure of magnetic domain motion and domain wall dynamics on timescales approaching femtoseconds, and spatial length scales of a few nanometers (310).

\section{Acknowledgments}

The authors thank P. Pourhossein Aghbolagh and R. Chiechi for discussions and instructions on the use of the ultramicrotome, as well as N. Rubiano da Silva, M. Möller, and J. Momand for help with TEM diffraction images, A. Feist for assistance with EELS spectroscopy, and the HZB for the allocation of synchrotron radiation beamtime at BESSY II. Synchrotron work was supported by F. Radu and T. Kachel. TD, CR, and RT thank the German Academic Exchange Service (DAAD) for support within the framework of the U4 University Network during TD's stay in Groningen. XZ acknowledges support from the National Natural Science Foundation of China (Grant No. 11204312) for work conducted at the High Magnetic Field Lab (Hefei). 
The work at Rutgers University was supported by the NSF under Grant No. NSF-DMREF1233349, and the work at Postech was supported by the Max Planck POSTECH/KOREA Research Initiative Program (Grant No. 2011-0031558) through NRF of Korea funded by MSIP. The research leading to these results has received funding from the European Community's Seventh Framework Programme (FP7/2007-2013) under Grant Agreement No. 312284. 

Chapter 5

\title{
Ultrafast nanoimaging of the order parameter in a structural phase transition
}

\author{
Th. Danz, T. Domröse, C. Ropers
}

Science 371, 371-374 (2021)

doi:10.1126/science.abd2774

Understanding microscopic processes in materials and devices that can be switched by light requires experimental access to dynamics on nanometer length and femtosecond time scales. Here, we introduce ultrafast dark-field electron microscopy to map the order parameter across a structural phase transition. We use ultrashort laser pulses to locally excite a $1 T-\mathrm{TaS}_{2}$ thin film, and image the transient state of the specimen by ultrashort electron pulses. A tailored dark-field aperture array allows us to track the evolution of charge-density wave domains in the material with simultaneous femtosecond temporal and 5-nm spatial resolution, elucidating relaxation pathways and domain wall dynamics. The unique benefits of selective contrast enhancement will inspire future beam shaping technology in ultrafast transmission electron microscopy.

This is the Accepted Version of the article published in Science 371, 371-374 (2021). 


\subsection{Main Text}

Optical control over physical and chemical properties of materials is a recurring motif from femtochemistry to ultrafast condensed matter physics. This broad interest is based on the impact of optical control strategies in current and future technology, such as data storage devices (311, 312), neuromorphic computing (313), photonic circuits (314), and energy conversion (2). In parallel, fundamental scientific discoveries reveal unique light-induced properties and correlation effects, involving coupled order parameters (315), metastable or hidden states $(14,26,258,316)$, superconductivity (317), changes in topology (10), and metal-insulator transitions $(170,175)$.

Typically, microscopic correlations evolve on femto- to picosecond time scales, a regime that is accessible by ultrafast measurement methodology. This approach enables spatially averaged probing of, e.g., electronic gaps (48), optical conductivity (318), magnetization (290), and instabilities against periodic lattice and charge-density modulations $(65,268)$. Functionality of devices, however, usually arises from nanoscale structuring or from interfacing different materials. This calls for experimental approaches capturing the dynamics in terms of a spatially dependent order parameter.

Recent experiments accomplished time-resolved mapping of the local free-carrier response in a correlated metal-insulator phase transition using near-field probing $(43,44)$. However, direct sensitivity to the spatiotemporal structural modifications governing the transition on the atomic scale remains an open challenge. Ultrafast transmission electron microscopy (UTEM) has proven a valuable tool to study lattice dynamics $(58,60,64,66,131,158,163)$, but does not provide direct contrast of the structural order parameter.

In this work, we demonstrate real-space imaging of charge-density wave (CDW) phases with nanometer spatial and femtosecond temporal resolution. Introducing a tailored ultrafast dark-field electron microscopy scheme, we obtain maps of the order parameter in a prototypical CDW system. We observe the formation, stabilization, and relaxation of CDW domains after optical excitation, and identify drastic differences between the emerging domain patterns for continuous-wave and pulsed illumination. Corroborated by time-dependent Ginzburg-Landau simulations, we discuss the non-equilibrium evolution of the order parameter near domain walls.

Specifically, we investigate the transition metal dichalcogenide $1 T-\mathrm{TaS}_{2}$. Owing to its lowdimensional character and strong correlation effects, this system is a promising target for nontrivial order parameter dynamics. It displays several CDW phases with both Mott and Peierls contributions and particularly complex orbital textures $(201,246)$. The CDW states and the 
A
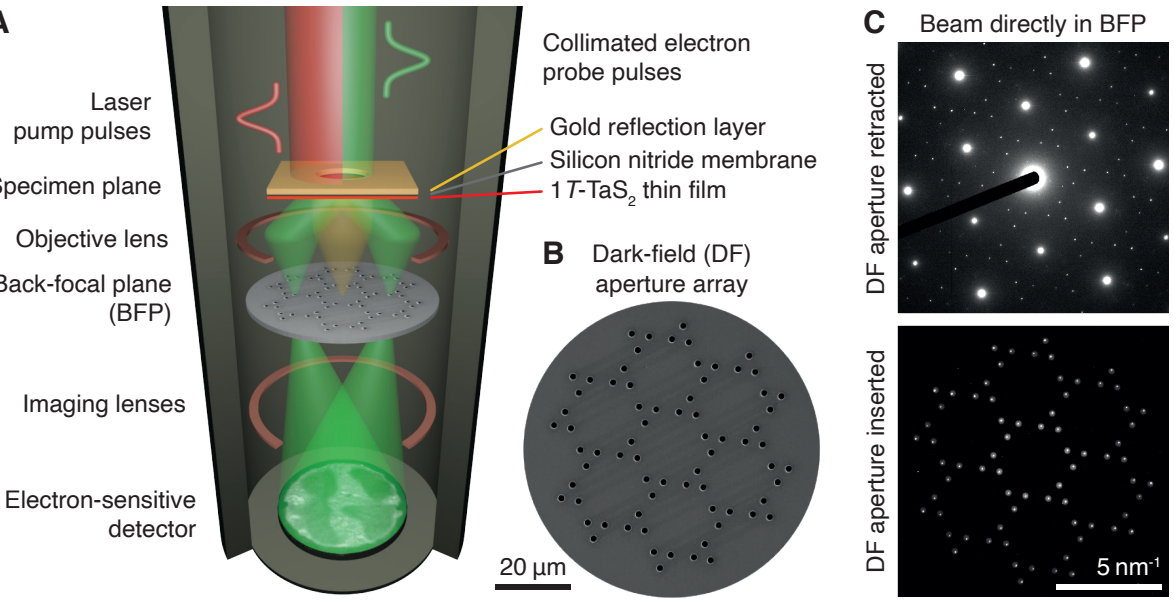

Figure 5.1: Dark-field imaging in the ultrafast transmission electron microscope. (A) Sectional drawing of the experimental setup. Electron (green) and optical pulses (red) are incident close to perpendicular on the specimen. (B) Scanning electron micrograph of the dark-field (DF) aperture array placed in the back-focal plane (BFP) in (A). (C) Electron diffractograms ${ }^{7}$ of the $1 T-\mathrm{TaS}_{2}$ thin film at room temperature with the DF aperture array shown in (B) retracted from (top) and inserted into the electron beam path (bottom). Only superstructure reflections are transmitted through the aperture array.

transitions between them have been the subject of various ultrafast spectroscopy and electron diffraction experiments in the past $(65,222,239,250,265-267)$.

Our study focuses on the room- and high-temperature phases of the material $(12,182,201$, 233). In all experiments, the sample is initially held at room temperature. Above a temperature of $T^{*}=353 \mathrm{~K}$, the material exhibits an incommensurate superstructure aligned with the underlying hexagonal lattice (IC CDW phase; in short IC phase hereafter). Below the phase transition temperature, the CDW transforms into a nearly commensurate superstructure with an angle of $\sim 12^{\circ}$ between the modulation wave vectors and the lattice directions, effectively reducing the symmetry of the material (NC CDW phase or NC phase). Each of the CDW phases is accompanied by a pronounced periodic lattice distortion (PLD). In electron diffraction images, the PLD of the NC phase is evident from satellite diffraction peaks arranged around the bright structural reflections (Fig. 5.1C, top). Owing to a threefold out-of-plane stacking periodicity in the NC phase, mainly second-order satellites are visible (182).

In real-space images, NC CDW contrast is obtained by means of dark-field (DF) imaging, i.e., by inserting a DF aperture into the back-focal plane of the microscope's objective lens. It was recognized early on that DF imaging is in principle suitable to map the spatial distribution of PLDs (12). However, standard circular DF apertures are too large for this purpose (12), and a single satellite diffraction peak is too weak due to brightness limitations of pulsed electron

\footnotetext{
${ }^{7}$ High-resolution prints of both diffractograms can be found in the appendix (Chapter A).
} 
beams ( $<1 \%$ intensity compared to structural reflections) (204). Thus, the present work uses focused ion beam etching to manufacture a tailored array of 72 small apertures, selecting the brightest second-order satellite reflections and blocking all other diffraction peaks (Fig. 5.1B) (319).

When the DF aperture array is inserted into the column of the electron microscope (Fig. 5.1C, bottom), only electrons scattered into NC satellite diffraction peaks can reach the detector and contribute to the image contrast, whereas electrons elastically scattered into main lattice peaks and IC diffraction peaks are blocked (Fig. 5.5). Accordingly, the DF images directly reflect the local order parameter $\phi$ of the NC CDW with a spatial resolution below $5 \mathrm{~nm}$ (319). Kinematical diffraction simulations based on the NC structure (182) indicate that the dependency between local image intensity and order parameter is close to quadratic with the specific set of reflections considered (319). Inelastic scattering contributions account for some background in the image even in the IC phase (233).

This approach now allows us to directly image CDW dynamics in the material (Movie 5.1), with a contrast unattainable in conventional bright-field imaging (Movie 5.2). In our timeresolved experiments, we use femtosecond laser pulses ( $800 \mathrm{~nm}$ center wavelength, $80 \mathrm{fs}$ FWHM duration, $420 \mathrm{kHz}$ repetition rate) to excite a freestanding $70 \mathrm{~nm} 1 T-\mathrm{TaS}_{2}$ film, and probe the transient state of the specimen using ultrashort electron pulses $(120 \mathrm{keV}$ electron energy, $530 \mathrm{fs}$ FWHM duration). After filtering using the DF aperture array, a spatial image is formed in the detector plane (see Fig. 5.1A, and Fig. 5.6 for more details). Spatially inhomogeneous excitation is required to trigger spatiotemporal dynamics in this experiment. Therefore, we use the circular gold aperture that supports the film as a means to structure the excitation profile by interference between the main beam and edge reflections (see supplementary text for further information). Additionally, the gold layer acts as a heat bath and prevents thermal load on the specimen outside the region of interest (319). This very local excitation and effective thermal coupling ensures reversibility of the observed dynamics at an unprecedented repetition rate for a structural phase transition.

Ultrafast DF images are shown in Fig. 5.2A as a function of optical pump/electron probe delay $\Delta t$ (319). Initially, the image within the circular aperture is flat with minor diffraction features and spatial variations of the NC CDW/PLD amplitude at room temperature. Directly after the temporal overlap of electron and laser pulses ('time-zero'), an inhomogeneous suppression of image intensity is evident with its shape governed by the excitation profile (Fig. 5.2E). Within two picoseconds, well-defined domains have emerged, and the boundaries separating bright from dark regions have become visibly sharper (Fig. 5.2D). On a 100 ps time scale, a slight growth 
A $\Delta t=-5 \mathrm{ps}$

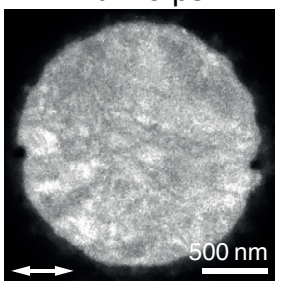

$130 \mathrm{ps}$

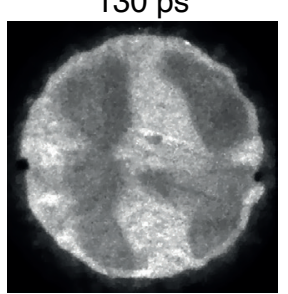

B

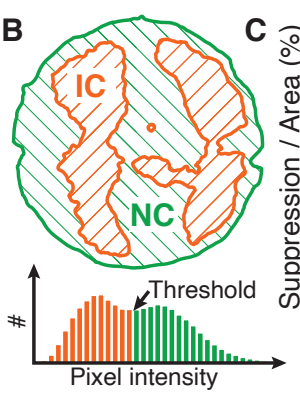

$0.75 \mathrm{ps}$

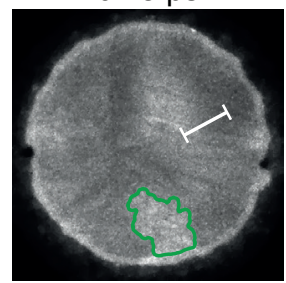

550 ps

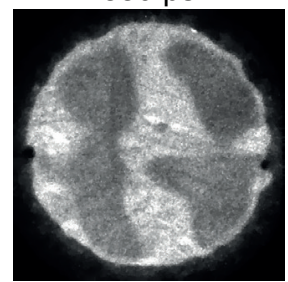

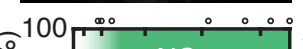

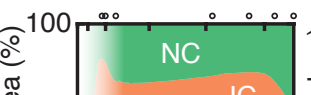

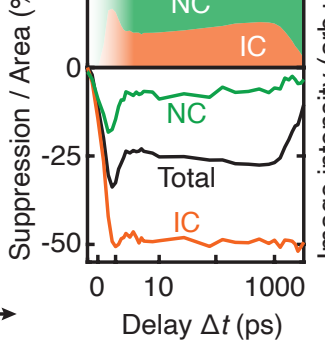

$1 \mathrm{ps}$

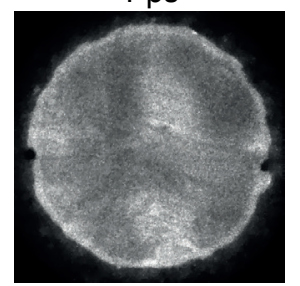

1500 ps
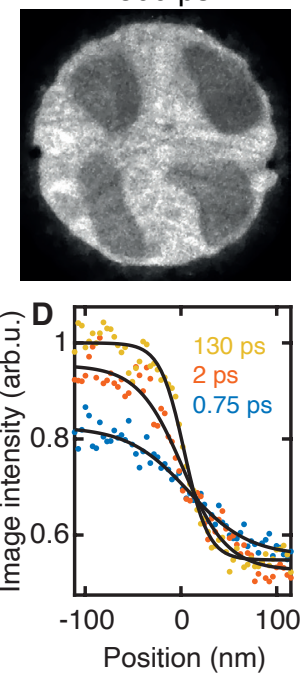

2 ps

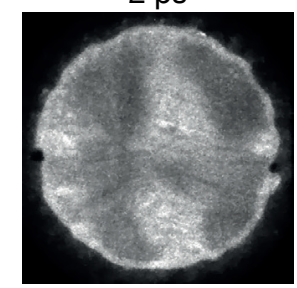

3141 ps

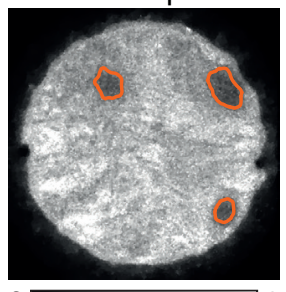

Image int. (arb.u.)

E

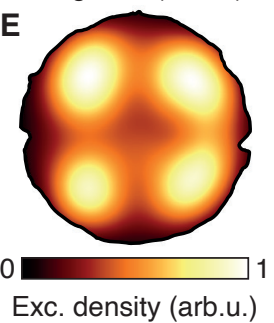

Figure 5.2: Ultrafast dark-field domain imaging of charge-density wave dynamics. (A) Ultrafast DF micrographs of transient domain configurations in the $1 T-\mathrm{TaS}_{2}$ film obtained in the laser pump/electron probe scheme $\left(2.6 \mathrm{~mJ} / \mathrm{cm}^{2}\right.$ pump fluence, linear pump polarization indicated by white arrow). Pump/probe delay steps were chosen so as to capture all major stages of the dynamics (see black circles above (C)). (B) Top: Image segmentation at $130 \mathrm{ps}$ delay time. Bottom: The segmentation threshold is determined from the intensity histogram of the full image series within the circular aperture. (C) Top: Area fractions of NC and IC regions after completed phase separation, as determined from the segmented images. Bottom: Average intensity of the image series within the entire aperture (black curve), and average intensity in weakly and strongly pumped regions (green/orange curve; evaluated regions are indicated in (A) using corresponding colors). (D) Exemplary profiles of NC/IC phase boundaries taken on the white line indicated in (A). (E) Spatial profile of the excitation density giving rise to the initial suppression pattern (see (319) and Fig. 5.7).

of the darkened regions and a further contrast sharpening is observed. Finally, these domains diminish in area, and a homogeneous NC contrast is re-established after a few nanoseconds.

The image series contains considerably more information than spatially averaged diffraction data alone. Specifically, we can individually analyze the PLD dynamics in weakly and strongly pumped regions, which either show a transient quench and recovery, or a persistent phase transformation with a full suppression within the temporal resolution (green and orange curves in Fig. 5.2C, bottom) (65). The practically binary contrast is a clear sign of a completed phase separation into distinct NC and IC regions no later than a few picoseconds after the pump. This allows us to carry out image segmentation in order to identify the temporal evolution of the 


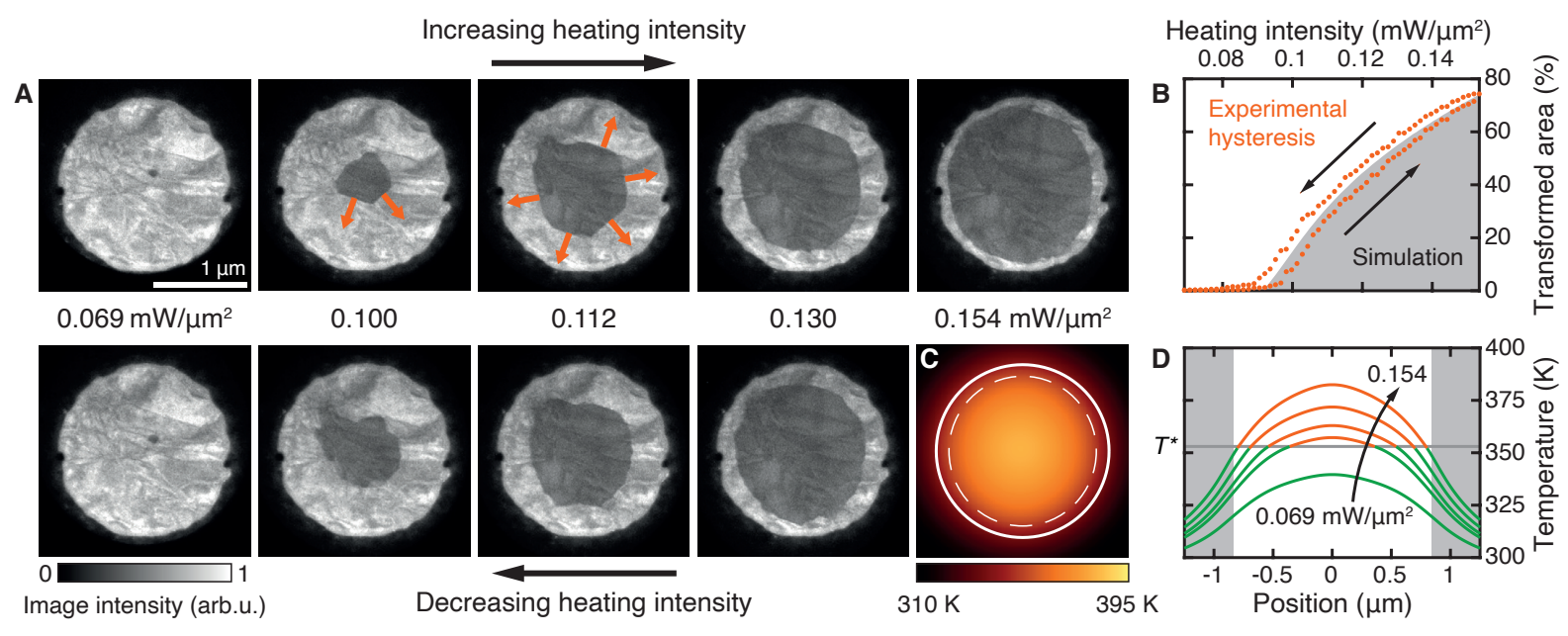

Figure 5.3: Dark-field domain imaging with continuous-wave laser excitation. (A) DF micrographs of laser intensity up-sweep and down-sweep. Orange arrows indicate phase boundaries oriented along preferential lattice directions. (B) Hysteretic behavior as extracted from the micrographs (orange dots). The characteristic intensity dependency is reproduced by heat transfer simulations (gray). (C) Temperature distribution simulated for a laser heating intensity of $0.154(9) \mathrm{mW} / \mu \mathrm{m}^{2}$ for comparison with the experimental result (solid line: edge of circular aperture; dashed line: edge of transformed area). (D) Radial profiles of the simulated temperature distribution in the film for the experimental laser intensities presented in (A). Green (orange) segment of the curves: temperature below (above) the phase transition temperature $T^{*}$. Shaded regions are outside the aperture. (See (319) for details on simulations.)

respective area fractions (Figs. 5.2B and 5.8). In particular, this analysis shows that the delayed additional suppression of the spatially averaged contrast (black curve in Fig. 5.2C), starting after a few picoseconds and lasting up to one nanosecond, is caused by the growth of the IC domains (see the moderate increase of the IC fraction in Fig. 5.2C, top).

The CDW phase pattern in the time-resolved experiment is a direct consequence of the spatiotemporal non-equilibrium, and strongly deviates from the situation observed under constant heating of the structure using a $532 \mathrm{~nm}$ continuous-wave laser (Fig. 5.3A and Movie 5.3): Upon increasing the laser heating intensity, an IC domain nucleates close to the center of the thin film, and grows until it almost fills the circular aperture. Decreasing the laser intensity results in a reduction of the switched area.

The steady-state phase pattern arises from the dynamical equilibrium between deposited and dissipated energy. Owing to efficient equilibration within the film compared to the thermal coupling to the support and heat bath, the optical excitation profile is not evident in this experiment. This provides us with a detailed picture of the sample's thermal state. We use a heat transfer simulation to predict the temperature distribution for each laser intensity (Fig. 5.3, B-D), and quantify the thermal boundary resistance between the film and the substrate. The value obtained falls well in line with those of related interfaces (319). 
A slight hysteresis of $<4 \mathrm{~K}$ is observed in the heating cycle (orange dots in Fig. 5.3B), a signature of the first-order character of the phase transition (12). On microscopic length scales, we identify two different mechanisms affecting the hysteretic behavior: The up-sweep reveals a preferential formation of $\mathrm{NC} / \mathrm{IC}$ phase boundaries along specific crystallographic directions (indicated by orange arrows in Fig. 5.3A; see supplementary text for details). On the other hand, local pinning sites visibly affect the down-sweep due to some degree of structural inhomogeneity (cf. Fig. 5.9, A-C).

The appearance of these effects only under continuous excitation is a result of very different energy and time scales governing the dynamics. The steady-state experiment is rather sensitive to local defects and structural anisotropy, as there is sufficient time for small spatial variations in the CDW free-energy landscape to determine the phase pattern. On the other hand, the time-resolved experiment is characterized by electron-lattice non-equilibrium involving strong thermal gradients.

In order to obtain a better understanding of the spatiotemporal CDW evolution and energy redistribution, we simulate the three-dimensional dynamics of the order parameter as well as the electron and lattice temperatures (319). Employing a Ginzburg-Landau model for a nonconserved order parameter, we capture the symmetry-breaking nature of the first-order NC/IC phase transition (see (214), model 'A'). As in the experiments, we do not separately analyze the individual wave vector components of the CDW. Accordingly, we consider the spatially varying PLD amplitude as a single, real-valued order parameter $\phi$ with a generic free-energy functional for a first-order phase transition, parametrized by the local electron temperature $T_{\mathrm{el}}$. The evolution of the electron and lattice temperatures is treated with coupled heat diffusion equations using the thermal parameters extracted from the steady-state experiment (319).

For values of $T_{\mathrm{el}}$ below the phase transition temperature $T^{*}$, the free-energy landscape exhibits a double-well shape (Fig. 5.4B). Initially, the simulation volume is in thermodynamical equilibrium with $\phi=1$, corresponding to one of the potential minima at room temperature. The optical excitation at time-zero is modeled as a jump in electronic temperature with the spatial distribution given by the excitation profile (Fig. 5.2E). In this highly non-equilibrium state with $T_{\text {el }}>T^{*}$ in large areas of the sample, the reshaped potential is close to parabolic with a single IC CDW minimum at $\phi=0$. The subsequent relaxation of the order parameter towards the high-symmetry state causes a global order parameter suppression at early times. Following a rapid electron-lattice energy transfer on a time scale of $350 \mathrm{fs}$ (65), the simulation in Fig. 5.4A and Movie 5.4 closely reproduces the phase separation and cooling observed in the experimental dataset (cf. Fig. 5.2A). 
A

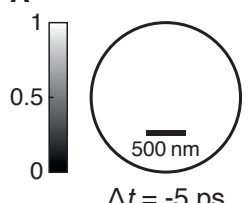

$\Delta t=-5 \mathrm{ps}$

B

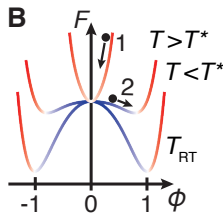

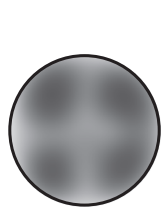

$0.1 \mathrm{ps}$

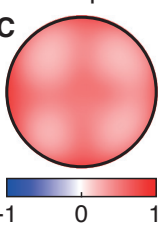

Dynamic evolution of simulated dark-field intensity $\phi^{2}(\Delta t)$
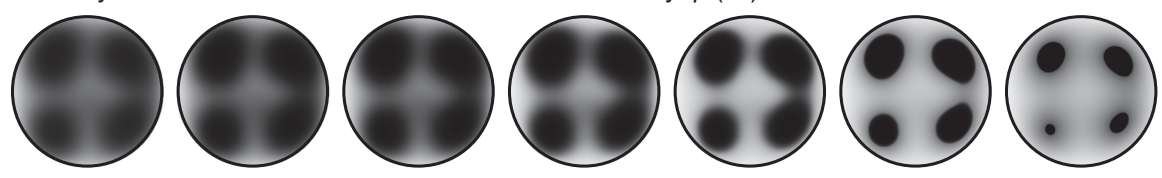

$0.75 \mathrm{ps}$
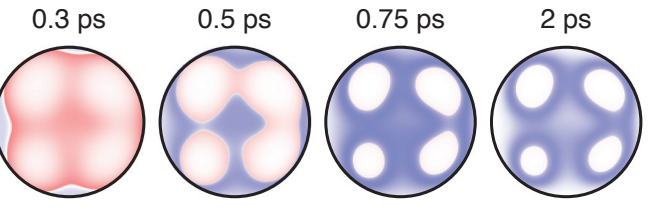

$5 \mathrm{ps}$

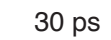

$3141 \mathrm{ps}$

Non-equilibrium contribution to order parameter $\phi^{2}(\Delta t)-\phi_{\mathrm{eq}}^{2}\left[T_{\mathrm{el}}(\Delta t)\right]$

Figure 5.4: Time-resolved Ginzburg-Landau simulations of optically induced order parameter dynamics.

(A) Series of simulated dark-field micrographs (squared order parameter $\phi^{2}$ projected along the specimen thickness in order to mimic DF image contrast). (B) Sketch of free-energy landscapes for different electron temperatures (color scale as described in $(\mathrm{C})$ ). $(\mathbf{C})$ Deviation of the squared order parameter from its local equilibrium value $\phi_{\text {eq }}^{2}$ (red/blue color: order parameter is larger/smaller than at the free-energy minimum). (See (319) for details on simulations.)

Local trajectories of the order parameter can be classified according to the magnitude of its non-equilibrium contribution (Fig. 5.4C and Movie 5.5). In regions with sufficient excitation density, the order parameter relaxes into the IC potential minimum within the electron-lattice coupling time (see point ' 1 ' in Fig. 5.4B). The spatiotemporal evolution of the corresponding four white patches adiabatically follows the reshaping of the potential on heat diffusion time scales. On the other hand, and as a consequence of the nearly flat potential just below the phase transition temperature, more weakly excited regions undergo a transient quench of the NC phase, relaxing on a few-picosecond time scale (point '2') (see also Fig. 5.10).

Close to the phase boundaries, the suppression of NC charge-ordering lasts until $30 \mathrm{ps}$ after excitation (see blue contours forming around the white patches after $2 \mathrm{ps}$ ), and a minor slowdown of the IC formation is observed as well (light red regions at $0.5 \mathrm{ps}$ ). These processes are a direct consequence of the delicate interplay between fast electron-lattice coupling and order parameter dynamics in a reshaped free-energy landscape, and ultimately result in the sharpening of domain walls observed in simulation and experiment (Fig. 5.11).

Our study illustrates how contrast enhancements by post-specimen $(95,320-322)$ or even pre-specimen beam shaping (323) allow for sensitivity to further degrees of freedom in complex materials, paving the way for other types of investigation. These possibilities include imaging of transient phonon populations (324), and particularly visualizing amplitude and phase modes in charge-density wave materials (204). Furthermore, spatially resolved investigations of topological defects in the charge-ordering of these materials may lead to a deeper understanding of phase formation kinetics $(222,266-268)$. Lastly, careful design of the beam shaping parameters 
may facilitate multi-phase imaging, or the simultaneous mapping of chiral mirror domains (265). Building upon these opportunities, ultrafast transmission electron microscopy will offer extraordinary perspectives for observing nanoscale dynamics in correlated materials.

\section{Acknowledgments}

The authors thank Murat Sivis for technical support in focused ion beam milling of specimen and DF aperture array, as well as Kai Rossnagel (University of Kiel) for supplying high-quality $1 T-\mathrm{TaS}_{2}$ crystals. We acknowledge assistance from the Göttingen UTEM Team and especially Tyler Harvey who designed the excitation beam path. Furthermore, we are grateful to Carolin Wichmann for providing the in-plane rotation specimen holder.

\section{Funding}

This work was funded by the Deutsche Forschungsgemeinschaft (DFG, German Research Foundation) in the Collaborative Research Center "Atomic scale control of energy conversion" (217133147/SFB 1073, project A05) and via resources from the Gottfried Wilhelm Leibniz Prize (RO 3936/4-1). Th.D. gratefully acknowledges a scholarship by the German Academic Scholarship Foundation.

\section{Author contributions}

Th.D. and Ti.D. conducted the experiments and analyzed the data. Th.D. designed and manufactured the DF aperture array. Th.D. and Ti.D. prepared the specimen and implemented the time-resolved simulations. Th.D. carried out the steady-state simulations. C.R. conceived and directed the study. All authors discussed the results and their interpretation and wrote the manuscript.

\section{Competing interests}

The authors declare no competing interests.

\section{Data and materials availability}

The data in the manuscript are available from GRO.data (325). 


\subsection{Materials and Methods}

\subsubsection{Ultrafast transmission electron microscopy in DF mode}

The Göttingen Ultrafast Transmission Electron Microscope (UTEM) is based on a commercial JEOL JEM-2100F Schottky field emission microscope, modified to allow for ultrashort electron pulse generation from a $\mathrm{ZrO} / \mathrm{W}$ Schottky field emitter tip using $400 \mathrm{~nm}$ ultrashort laser pulses. This high-coherence ultrafast electron source enables the investigation of spatiotemporal dynamics in a laser pump/electron probe scheme with sub-nanometer spatial resolution, $0.6 \mathrm{eV}$ energy resolution, and down to $200 \mathrm{fs}$ temporal resolution. Technical details on the instrument are given in $(66)$.

In this study, the $800 \mathrm{~nm}$ pump pulses arrive at the specimen near normal incidence $\left(\sim 6^{\circ}\right)$ and are focused down to $15 \mu \mathrm{m}$ FWHM using an aspherical lens. A collimated electron beam illuminates the specimen with a $2.4 \mu \mathrm{m}$ spot diameter (slightly larger than the $1.8 \mu \mathrm{m}$ circular gold aperture supporting the membrane; see subsection "Specimen preparation and characterization"). The specimen is mounted in an in-plane rotation specimen holder (Fischione Model 2040).

In DF mode, the DF aperture array filters NC CDW wave vector components in the back-focal plane of the objective lens where a first electron diffraction pattern is formed (see extended schematic in Fig. 5.6A). Finally, the real-space image is captured either on a direct detection camera (Direct Electron DE-16 in "counting mode"), or a conventional scintillator-coupled CCD (Gatan UltraScan 4000). The CCD detector has only been used in the steady-state experiment (Fig. 5.3A and Movie 5.3).

\subsubsection{Manufacturing of DF aperture array and theoretical resolution limit}

In order to introduce the DF aperture array into the microscope, we constructed an objective lens aperture holder that accommodates two standard-sized $3 \mathrm{~mm}$ silicon nitride grids for transmission electron microscopy (TEM) (Fig. 5.6, B and C). Each TEM grid (Silson; $50 \mathrm{~nm} \mathrm{Si}_{3} \mathrm{~N}_{4}$ film thickness, $500 \mu \mathrm{m}$ window size) is covered with a polycrystalline, sufficiently electron-opaque gold film of $600 \mathrm{~nm}$ nominal thickness by magnetron sputtering. Subsequently, we prepared the aperture array (Fig. 5.6D) using focused ion beam milling (FEI Nova NanoLab 600 DualBeam).

Based on Fig. 5.6E, we determine an actual gold film thickness of $780 \mathrm{~nm}$ and a hole diameter of $1.8 \mu \mathrm{m}$. Given the corresponding passband in reciprocal space, we estimate an achievable spatial image resolution in DF mode better than $5 \mathrm{~nm}$. 


\subsubsection{Specimen preparation and characterization}

We used magnetron sputtering to deposit a $5 \mathrm{~nm}$ titanium adhesion layer and a $200 \mathrm{~nm}$ gold film on the backside of standard-sized $3 \mathrm{~mm}$ silicon nitride TEM grids (Norcada; $30 \mathrm{~nm} \mathrm{Si}_{3} \mathrm{~N}_{4}$ film thickness, $10 \mu \mathrm{m}$ window size). Afterwards, we created a $1.85 \mu \mathrm{m}$ circular through-hole in the center of the $10 \mu \mathrm{m}$ window by focused ion beam milling, and deposited a $1 T-\mathrm{TaS}_{2}$ flake obtained by ultramicrotomy on top (Leica Ultracut UCT with DiATOME Ultra $45^{\circ}$ diamond knife; $50 \mathrm{~nm}$ nominal thickness) (67).

Figure 5.9, A-C shows electron micrographs of the specimen structure obtained using different imaging modes with a continuous electron beam. In order to characterize the actual specimen thickness, we use STEM-EELS (scanning transmission electron microscopy with an electron energy loss spectrum recorded per image pixel) at $200 \mathrm{kV}$ acceleration voltage $(\sim 13.0 \mathrm{mrad}$ convergence angle, $\sim 14.3 \mathrm{mrad}$ collection angle), and extract the local specimen thickness per pixel in units of the electron mean free path (MFP) (77). Using the formula derived by Iakoubovskii et al. (111), we calculate the MFP of $1 T-\mathrm{TaS}_{2}$, gold, and the silicon nitride membrane based on the convergence angle, the collection angle, and the respective material densities (neglecting the thin titanium adhesion layer) $(326,327)$. This results in an MFP of $126 \mathrm{~nm}$ for $1 T-\mathrm{TaS}_{2}, 153 \mathrm{~nm}$ for the silicon nitride membrane, and $99 \mathrm{~nm}$ for gold. Treating the $30 \mathrm{~nm}$ silicon nitride thickness as fixed, we obtain actual layer thicknesses of $70 \mathrm{~nm}$ for the free-standing $1 T-\mathrm{TaS}_{2}$ flake, and $145 \mathrm{~nm}$ for the gold film (averaged along the image edges) (Fig. 5.9, D and E).

\subsubsection{Image post-processing, image segmentation, and delay curves}

Due to limited maximum exposure times of the cameras, each individual image taken consists of a certain number of frames (between one and 60, depending on the specific experiment) that are added up to obtain the final image. For data taken with the direct detection camera under low-dose conditions and presented in the main text, effective integration times per image range between $11 \mathrm{~min}$ (Fig. 5.2C) and $65 \mathrm{~min}$ (Fig. 5.2A). Fluctuations in beam current over time are compensated by image normalization based on the intensity of reference images taken at regular intervals and under identical experimental conditions (before time-zero).

After removal of fixed-pattern noise, we use total variation denoising to reduce the presence of shot noise in the experimental image series while retaining edges (328), especially those between regions of different CDW phases. Additionally, we align the individual images in each 
image series using the edge of the circular gold aperture as a reference in order to compensate for potential specimen drift during the experiment.

An image segmentation approach is applied to convert the NC/IC CDW image contrast to binary masks of the two phases, obtaining their respective occupied areas. In order to calculate the image intensity threshold of the full image series, we use Otsu's method which minimizes the intra-class variance and maximizes the inter-class variance of bright and dark pixels (329). After applying the threshold to the image series, we remove NC and IC regions from the binary masks whose area is smaller than a certain threshold in order to reduce fragmentation of the masks.

Finally, delay curves for weakly and strongly pumped regions (green/orange curve in Fig. 5.2C) are derived by spatially averaging the image intensity over the regions indicated in Fig. 5.2A at 0.75 and $3141 \mathrm{ps.} \mathrm{The} \mathrm{average} \mathrm{signal} \mathrm{(black} \mathrm{curve)} \mathrm{is} \mathrm{obtained} \mathrm{from} \mathrm{the} \mathrm{total} \mathrm{counts} \mathrm{inside}$ the circular aperture. All logarithmic delay axes in this work are generated using a symmetric logarithm transformation in order to be able to include data before and close to time-zero as well (330).

\subsubsection{Extraction of excitation profile from experimental data}

The spatial profile of the excitation density shown in Fig. 5.2E is extracted from an ultrafast DF image series recorded as a function of pump fluence at a fixed pump/probe delay of $0.75 \mathrm{ps}$ (Fig. 5.7). After image segmentation, we assign the peak excitation density $I_{1}$ to the region that is being transformed into the IC phase already at the lowest pump fluence $F_{1}$. For all areas of the specimen transformed into the IC phase at a fluence $F_{i}$, we extract an excitation density $I_{i}=I_{1} \cdot F_{1} / F_{i}$. The final excitation profile is obtained after Gaussian smoothing and normalizing to the average within the gold aperture.

\subsubsection{Kinematical diffraction simulation of the NC phase}

The presence of the nearly commensurate $\mathrm{CDW}$ in the $\mathrm{NC}$ phase of $1 T-\mathrm{TaS}_{2}$ leads to superlattice reflections that appear in the diffraction pattern in addition to the structural Bragg reflections. Overhauser described the structure factor for a sinusoidal charge-density modulation in a linear chain of atoms in (204). However, for a three-dimensional, nearly commensurate CDW as in $1 T-\mathrm{TaS}_{2}$, and in the presence of possible higher-harmonic contributions to the charge-density modulation, an analytic calculation of structure factors becomes much more difficult. 
In order to compare experimental data with simulated order parameter dynamics, we need access to the dependency between the order parameter of the NC phase (the CDW/PLD amplitude) and the total intensity of the 72 superlattice reflections that contribute to the DF image contrast. We use a dataset of atom positions by Spijkerman et al. which includes a number of different harmonics to describe the modulation of both tantalum and sulfur positions in the material (182). The atomic scattering factors of tantalum and sulfur are included in the calculations (99). Additionally, we approximate the NC CDW structure by a commensurate one with a size of $147 \times 147 \times 3$ undistorted unit cells.

In this setting, we calculate the structure factors of the 72 reflections as a function of CDW amplitude by linearly scaling the harmonic coefficients between zero and their full value. A fit of CDW amplitude vs. simulated DF image intensity reveals a power law scaling with an exponent of approximately 1.92. For the sake of simplicity, we use a value of 2 in the main text and Fig. 5.4.

\subsubsection{Finite element simulations: General setup}

The simulation results outlined in the main text are obtained using finite element simulations in COMSOL Multiphysics (331). All simulations are conducted using the same model of the specimen but varying meshes and sizes of the simulation volume according to the requirements of each simulation step.

The lateral dimensions of the full simulation geometry cover the area of the $10 \mu \mathrm{m}$ silicon nitride window (Fig. 5.9F). Outside of the window, sufficient thermal coupling to the heat bath/200 $\mu \mathrm{m}$ thick silicon frame of the TEM grid is assumed. $1 T-\mathrm{TaS}_{2}$ and gold layers of equal size are positioned on the bottom and top of the silicon nitride membrane, respectively. Gold and silicon nitride material is removed within the circular aperture. We neglect the presence of the thin titanium adhesion layer in the model. All layer thicknesses and geometry dimensions are defined as determined from the actual specimen (see subsection "Specimen preparation and characterization"). Throughout the different simulations, we use material properties taken from the references listed in Table 5.1. For the thermal properties, we use temperature-dependent values where possible, and constant extrapolation outside the available data range. The heat bath is at room temperature at all times $\left(T_{\mathrm{RT}}=293.15 \mathrm{~K}\right)$. 


\subsubsection{Finite element simulations: Field calculations}

Initially, we calculate the absorption behavior for both optical pump wavelengths in this study (532 nm and $800 \mathrm{~nm}$ ) using the "Wave Optics" module of COMSOL in order to derive the absorbed laser power per unit volume at any point of the specimen. In order to reduce computational complexity, we only calculate the optical fields inside and close to the circular aperture (two wavelengths from aperture edge to simulation boundary). Additionally, we harness the symmetry of the model by reducing the simulation volume to one quadrant of the geometry and setting appropriate boundary conditions for the field ("Perfect Magnetic Conductor" at stitch boundaries where the electric field is tangential to the boundary, and "Perfect Electric Conductor" where the electric field is normal to the boundary).

The simulation itself is carried out using a scattered field formulation for the electric field. In a first step, we calculate the fields resulting from a plane wave impinging on the specimen stack in perpendicular incidence with the circular aperture removed ("background field"). In the second step, we calculate the "scattered field" of the full structure including the circular aperture, taking the background field into account. The sum of background field and scattered field then gives a precise solution for the field distribution inside and around the structure.

\subsubsection{Finite element simulations: Steady-state experiment}

We simulate the domain pattern of the steady-state experiment using the "Heat Transfer in Solids" module of COMSOL. We now take the full size of the simulation geometry into account, because we can only assume sufficient thermal coupling to the heat bath at the edges of the silicon nitride window. However, we are still able to reduce the model to a single quadrant due to symmetry (using "Thermal insulation" boundary conditions at the stitch boundaries). The absorbed laser power per unit volume at a pump wavelength of $532 \mathrm{~nm}$ (see previous subsection) is entered as a three-dimensional heat source. Due to the layered nature of $1 T-\mathrm{TaS}_{2}$, we assume a reduction of thermal conductivity by a factor of $\sim 3$ perpendicular to the layers (332). Then we use both the incident laser intensity and the thermal conductivity of the silicon nitride membrane to fit the simulation results for the transformed area vs. incident laser intensity curve to the experimental data (Fig. 5.3B).

The obtained value for the heat conductivity of the silicon nitride layer amounts to $0.9(2) \%$ of the silicon nitride bulk value; thus, it is dominated by the quality of the $1 T-\mathrm{TaS}_{2} / \mathrm{silicon}$ nitride and silicon nitride/gold interfaces. From this, we deduce a total interfacial thermal resistance of $\sim 0.7 \cdot 10^{-6} \mathrm{~m}^{2} \mathrm{~K} / \mathrm{W}$, which falls well in line with results for the thermal contact between a 
$\mathrm{MoS}_{2}$ flake and platinum electrodes (333). From the fit, we obtain a reasonable value of $20 \mu \mathrm{m}$ FWHM for the focused laser spot size at $532 \mathrm{~nm}$.

\subsubsection{Finite element simulations: Heat transfer in ultrafast experiment}

Based on the specimen model as refined in the steady-state experiment, we simulate the timedependent heat transfer underlying the specimen response in the ultrafast experiment. We describe the non-equilibrium state of the $1 T-\mathrm{TaS}_{2}$ layer in the first few picoseconds using a twotemperature approach for both electron and lattice systems, while assuming full electron-lattice equilibration at all times in gold and silicon nitride.

We consider a linear electronic heat capacity $C_{\mathrm{el}}=\gamma \cdot T_{\mathrm{el}}$ for the $1 T-\mathrm{TaS}_{2}$ layer with a linear coefficient of $\gamma=8.5 \mathrm{~mJ} / \mathrm{mol} / \mathrm{K}^{2}$ (cf. (334)), and we choose the lattice heat capacity $C_{\mathrm{ph}}$ such that we retain $C_{\mathrm{total}}=C_{\mathrm{el}}+C_{\mathrm{ph}}$ as given in (335). Electron and lattice thermal conductivities are distributed between both subsystems as given in (336). The electron-lattice coupling constant is selected such that subsystems equilibrate on a timescale of $\tau_{\mathrm{el}-\mathrm{ph}}=350 \mathrm{fs}(65)$, representing the rapid energy transfer from the electron system to a subset of strongly coupled phonon modes linked to the CDW/PLD formation. A timescale of $\sim 4 \mathrm{ps}$ has been attributed to the actual equilibration of electron and lattice temperature in the system (65).

The COMSOL field calculations do not closely reproduce the observed excitation pattern due to the precise shape of the actual gold aperture in the experiments. Instead, we combine the depth dependency from the field calculations with the spatial profile of the excitation density as extracted from experimental data (shown in Fig. 5.2E; see subsection "Extraction of excitation profile from experimental data"). This excitation pattern breaks the four-fold symmetry of the model; thus, we simulate the full $10 \mu \mathrm{m} \cdot 10 \mu \mathrm{m}$ square of the silicon nitride membrane in this step.

Based on the assumption that the experimentally observed order parameter evolution essentially follows the temperature distribution at late times, we tune the mean excitation fluence in the simulations such that the regions with temperatures $T \geq 353 \mathrm{~K}$ reproduce the experimentally observed pattern at $3141 \mathrm{~ns}$ (Fig. 5.2A). This leads to a fluence of $1.3 \mathrm{~mJ} / \mathrm{cm}^{2}$ in the simulations, while the estimated fluence in the experiments is $2.6 \mathrm{~mJ} / \mathrm{cm}^{2}$. We attribute this difference to the uncertainty in determining the experimental pump spot diameter. In order to reproduce the experimental data at early times as well, we assume an instantaneous thermal equilibration of the electron temperature over the specimen thickness due to ultrafast heat transport by highly excited electrons (337). 


\subsubsection{Finite element simulations: Time-dependent Ginzburg-Landau approach}

Based on the ultrafast heat transfer simulations, we implement a simulation of the order parameter dynamics in the $1 T-\mathrm{TaS}_{2}$ layer using a time-dependent Ginzburg-Landau approach. We use a partial differential equation based on model 'A' by Hohenberg and Halperin (214), describing the spatiotemporal evolution of a non-conserved order parameter:

$$
\frac{\mathrm{d} \phi}{\mathrm{d} t}=d \cdot \Delta \phi-\xi \cdot \frac{\partial F}{\partial \phi}
$$

Here, $d$ is a measure of the energy related to the formation of a domain wall between NC and IC phases, and $\xi$ is a global time scale of the free-energy landscape $F$. The behavior of the free-energy functional

$$
F\left(\phi, T_{\mathrm{el}}\right)=F_{0}+\alpha_{0} \cdot\left(T_{\mathrm{el}}-T_{\mathrm{C}}\right) \cdot \phi^{2}+\frac{\beta}{2} \cdot \phi^{4}+\frac{\gamma}{3} \cdot \phi^{6}
$$

models a first-order phase transition in the order parameter $\phi$ as a function of the local electron temperature $T_{\mathrm{el}}$ with $\alpha_{0}>0, \beta<0$, and $\gamma>0$ (217). The critical temperature $T_{\mathrm{C}}$ denotes the low-temperature side of the hysteresis loop, while

$$
T_{1}=\frac{\beta^{2}}{4 \alpha_{0} \gamma}+T_{\mathrm{C}}
$$

is the high-temperature end of the bistability regime. The potential minima attributed to NC and IC phase, respectively, are as follows:

$$
\begin{array}{clrl}
\phi_{\mathrm{NC}}\left(T_{\mathrm{el}}\right) & = \pm \sqrt{\frac{-\beta+\sqrt{\beta^{2}-4 \alpha_{0}\left(T_{\mathrm{el}}-T_{\mathrm{C}}\right) \gamma}}{2 \gamma}} & \text { for } & T_{\mathrm{el}}<T_{1} \\
\phi_{\mathrm{IC}} & =0 & \text { for } & T_{\mathrm{el}}>T_{\mathrm{C}}
\end{array}
$$

The bistability of $\phi_{\mathrm{NC}}$ reflects the two possible alignments of the NC CDW with the lattice and the symmetry-breaking character of the phase transition. At the phase transition temperature of $T^{*}=353 \mathrm{~K}$, the free energies $F\left(\phi_{\mathrm{NC}}\right)$ and $F\left(\phi_{\mathrm{IC}}\right)$ of both CDW phases are equal:

$$
T^{*}=\frac{3 \beta^{2}}{16 \alpha_{0} \gamma}+T_{\mathrm{C}}
$$


The entropy $S=-\mathrm{d} F / \mathrm{d} T_{\text {el }}$ of both states is given by:

$$
\begin{aligned}
S_{\mathrm{NC}}\left(T_{\mathrm{el}}\right) & =S_{0}-\alpha_{0} \cdot \phi_{\mathrm{NC}}\left(T_{\mathrm{el}}\right)^{2} & & \text { for } T_{\mathrm{el}}<T_{1}, \\
S_{\mathrm{IC}} & =S_{0} & \text { for } & T_{\mathrm{el}}>T_{\mathrm{C}} .
\end{aligned}
$$

For the heat capacity $C=T_{\mathrm{el}} \cdot\left(\mathrm{d} S / \mathrm{d} T_{\mathrm{el}}\right)$, we find:

$$
\begin{aligned}
C_{\mathrm{NC}}\left(T_{\mathrm{el}}\right) & =C_{0}+\frac{\alpha_{0}^{2} \cdot T_{\mathrm{el}}}{\sqrt{\beta^{2}-4 \alpha_{0}\left(T_{\mathrm{el}}-T_{\mathrm{C}}\right) \gamma}} & \text { for } \quad T_{\mathrm{el}}<T_{1}, \\
C_{\mathrm{IC}} & =C_{0} & \text { for } \quad T_{\mathrm{el}}>T_{\mathrm{C}} .
\end{aligned}
$$

Due to its first-order character, the phase transition is associated with a finite latent heat $\Delta H$ and a jump in heat capacity $\Delta C$ (both evaluated at $T^{*}$ ):

$$
\begin{aligned}
& \Delta C\left(T^{*}\right)=C_{\mathrm{IC}}\left(T^{*}\right)-C_{\mathrm{NC}}\left(T^{*}\right)=\frac{2 \alpha_{0}^{2}}{\beta} T^{*}, \\
& \Delta H\left(T^{*}\right)=\left[S_{\mathrm{IC}}\left(T^{*}\right)-S_{\mathrm{NC}}\left(T^{*}\right)\right] \cdot T^{*}=-\frac{3}{4} \frac{\alpha_{0} \beta}{\gamma} T^{*} .
\end{aligned}
$$

By normalizing the NC CDW potential minima to $\phi_{\mathrm{NC}}\left(T_{\mathrm{RT}}\right)=1$ at room temperature, and using literature values for $\Delta C(335)$ and $\Delta H(12)$, we can derive values for the constants $\alpha_{0}, \beta$, and $\gamma$ :

$$
\begin{aligned}
\alpha_{0} & =8.04 \mathrm{~J} / \mathrm{mol} / \mathrm{K}, \\
\beta & =-151 \mathrm{~J} / \mathrm{mol}, \\
\gamma & =626 \mathrm{~J} / \mathrm{mol} .
\end{aligned}
$$

This leads to a width of hysteresis $\Delta T=T_{1}-T_{\mathrm{C}}=1.1 \mathrm{~K}$ which is very well compatible with our upper estimate of $4 \mathrm{~K}$ (see main text).

In our simulations, the parameter $d$ effectively prevents the formation of step-like phase boundaries between NC and IC CDW regions. Given the spatial resolution in measuring the phase boundary width in the steady-state experiment, we deduce an upper limit of $d=1 \mathrm{~nm}^{2} / \mathrm{ps}$. Smaller values of $d$ only have a moderate effect on the ultrafast order parameter dynamics on the length scales considered in this work, such that the specific selection of $d$ is not of great significance. We choose a value of $0.1 \mathrm{~nm}^{2} / \mathrm{ps}$ in our simulations (cf. Fig. 5.11A). The global time scale $\xi$ is the only remaining parameter in order to fit the magnitude of the initial order 
parameter suppression during optical excitation. A value of $\xi=5 \cdot 10^{-4} \mathrm{~mol} / \mathrm{J} / \mathrm{ps}$ is used in the simulations presented in Fig. 5.4.

Initially, the simulation volume is in the room-temperature potential minimum of the NC phase with $\phi_{\mathrm{NC}}\left(T_{\mathrm{RT}}\right)=1$. In the experiments, the confinement of the optical excitation to the field of view of the electron beam and the good coupling to the heat bath suppress independent nucleation of the NC phase. The chiral state of the re-established NC phase is therefore determined by the initial NC orientation. Generally, the presence of the second NC potential minimum would allow for modeling of the probabilistic appearance of "mirror domains" (265). However, the deterministic nature of our simulation prohibits this kind of relaxation dynamics in agreement with our experimental observations.

\subsection{Supplementary Text}

\subsubsection{Structured excitation profile inside the circular gold aperture}

In the main text, we state that the precise shape of the (almost) circular aperture on top of the specimen determines the spatial profile of the excitation density visible in the ultrafast experiment (Fig. 5.2A) and gives rise to a four-lobe excitation pattern as shown in Fig. 5.2E. This behavior arises due to the symmetry breaking by the linear optical polarization.

This interpretation is backed up by the fact that the phase pattern in the steady-state experiment bears no sign of the four-lobe characteristics. Accordingly, the excitation profile seems not to be related to a significant degree of specimen inhomogeneity, e.g. in the strain distribution. Instead, the pattern is almost completely governed by the stationary thermal state induced by the heating laser. Two small exceptions of that behavior are noted in the main text (local pinning sites and preferential formation of NC/IC phase boundaries along specific crystallographic directions). These may play a minor role as well for explaining the subtle differences in the domain shapes between ultrafast experiment and time-resolved simulation. Additional shape irregularities that are not reproduced in the simulations may stem from the fact that the extracted excitation profile is not sufficiently well resolved.

We further support our interpretation with an additional dataset. In Fig. 5.12, we provide DF images at a fixed pump/probe delay and pump fluence, but with a varying angle of linear polarization between $0^{\circ}$ and $45^{\circ}$. Comparing the images at $0^{\circ}$ and $45^{\circ}$ linear polarization, one can see that a part of the four-lobe pattern is rotated by $45^{\circ}$, demonstrating the influence of the pump field on the domain pattern. 
Some of the additional complexity that is visible in the pattern at $45^{\circ}$ arises from the fact that the polarization rotation is done outside of the column of the electron microscope. After polarization manipulation, the beam passes additional mirrors with a combination of $\mathrm{s}$ - and $\mathrm{p}$ polarization. This leads to an additional phase-shift per reflection between s- and p-components of the beam, and thus a deviation from linear towards elliptic polarization characteristics.

\subsubsection{Orientation of NC/IC phase boundaries along preferential directions}

In the steady-state experiment, we observe the formation of NC/IC domain walls along preferential directions that reflect the hexagonal symmetry of the underlying lattice. In order to analyze these preferential directions, we first determine the relative rotation between diffraction and imaging modes of our TEM with the help of an anisotropically etched silicon frame. Most of the rotation is already compensated in the imaging system of the TEM, so that the residual offset only amounts to $1.2(4)^{\circ}$.

From the steady-state DF image series, we select four images that show the most pronounced formation of straight phase boundaries. After exclusion of those phase boundaries that seem to exhibit small "steps", we extract the angles (modulo $60^{\circ}$ ) of the phase boundaries indicated in Fig. 5.13A. The resulting angle interval is drawn in gray over the diffraction image of the NC phase in Fig. 5.13B. The obtained direction seems to indicate some correspondence with the $\{11 \overline{2} 0\}$ planes of the underlying hexagonal lattice, but future work will be required to associate this with a particular microscopic origin. 


\subsection{Supplementary Figures}

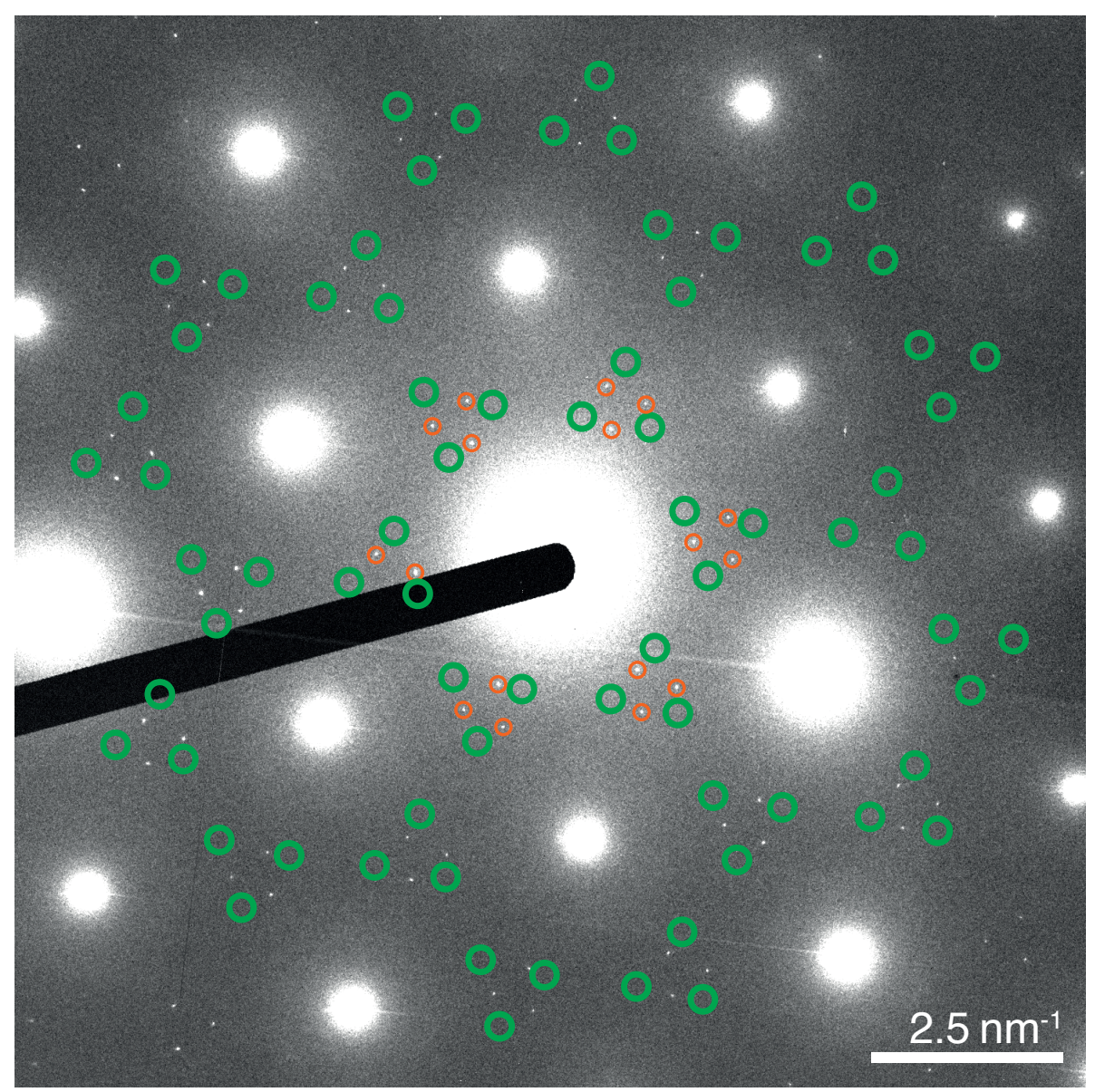

Figure 5.5: Diffraction pattern ${ }^{8}$ of the IC phase of $1 T-\mathrm{TaS}_{2}$.

The thin film is heated from room temperature using $0.071 \mathrm{~mW} / \mu \mathrm{m}^{2}$ continuous-wave laser intensity. Secondorder IC superstructure reflections are visible along with bright structural reflections and some inelastic background. As in the NC phase, first-order reflections are forbidden due to stacking periodicity (233). For clarity, those IC reflections that appear closest to the direct beam have been highlighted by orange circles. Position and size of the individual apertures in the DF aperture array are indicated by green circles (cf. Fig. 5.1B). The superstructure peaks of the IC phase appear close to the positions of the individual apertures in the DF array, however, there is no overlap between them.

\footnotetext{
${ }^{8} \mathrm{An}$ unmarked high-resolution print of the diffractogram can be found in the appendix (Chapter A).
} 

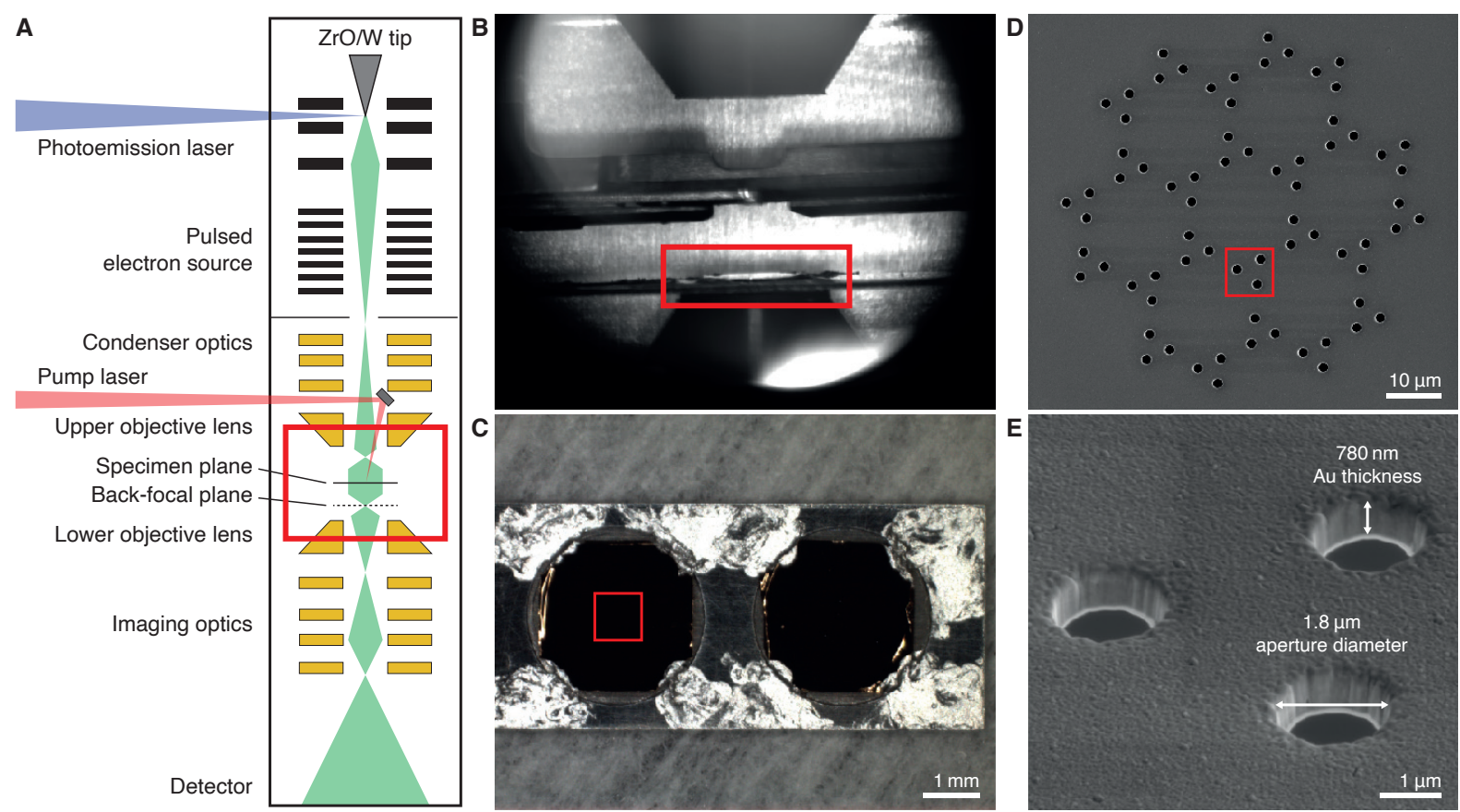

Figure 5.6: Extended schematic of the experiment and the DF aperture array.

(A) Schematic of the Göttingen Ultrafast Transmission Electron Microscope (UTEM) in the configuration used in this study. Red boxes indicate the field of view of the subsequent subfigure. (B) Optical camera image of the objective lens gap. Specimen holder and custom aperture holder are visible in the gap. The $3 \mathrm{~mm}$ DF aperture frame is marked. (C) Two DF aperture frames installed in the aperture holder. (D) Scanning electron micrograph of the DF aperture array taken after ion milling of the hole pattern. (E) Cross-sectional view of a hole triplet $\left(52^{\circ}\right.$ specimen tilt; image taken on another DF aperture array from the same batch). 


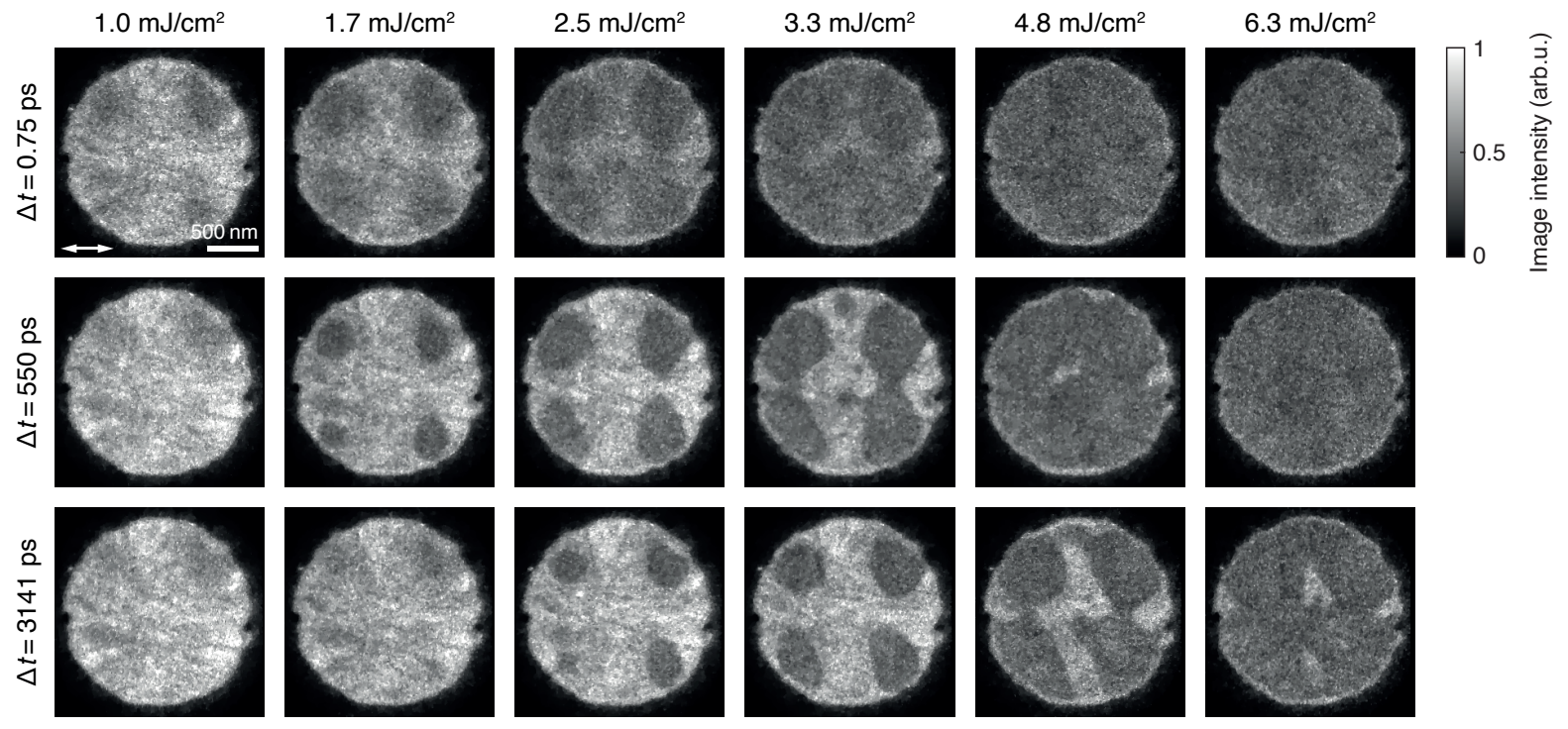

Figure 5.7: Ultrafast DF images as a function of laser fluence at fixed pump/probe delays.

Images taken with horizontal pump polarization as indicated by white arrow (11 min integration time per frame at $0.75 \mathrm{ps}$ delay, $5.5 \mathrm{~min}$ integration time at $550 \mathrm{ps}$ and $3141 \mathrm{ps}$ ). The spatial profile of the excitation density has been extracted from this dataset (see subsection "Extraction of excitation profile from experimental data"). 


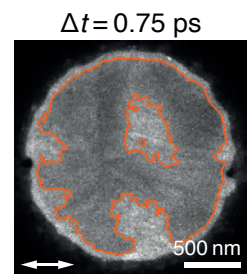

130 ps

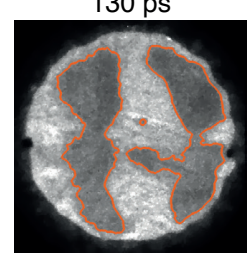

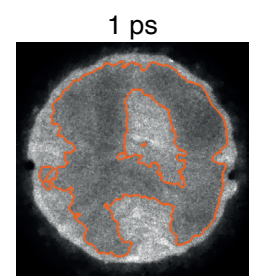

550 ps

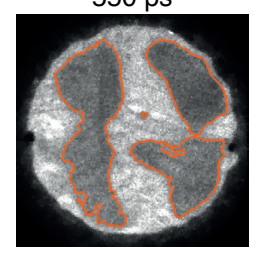

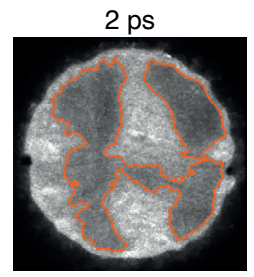

1500 ps

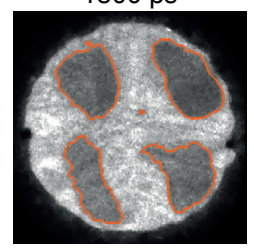

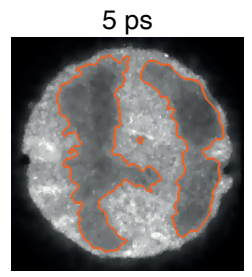

2500 ps

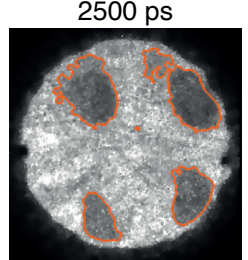

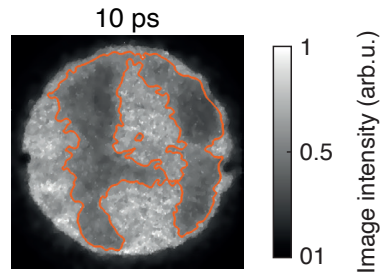

3141 ps

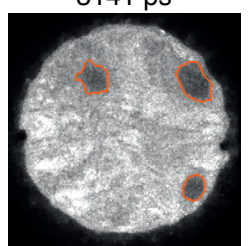

Figure 5.8: Demonstration of image segmentation.

Demonstration of the image segmentation approach based on the image series presented in Fig. 5.2A. The contrast of the additional images at 5, 10 and $2500 \mathrm{ps}$ has been adjusted in order to accommodate for a different intensity ratio of bright and dark regions (due to a slight drift of the DF aperture array during image acquisition). 
A

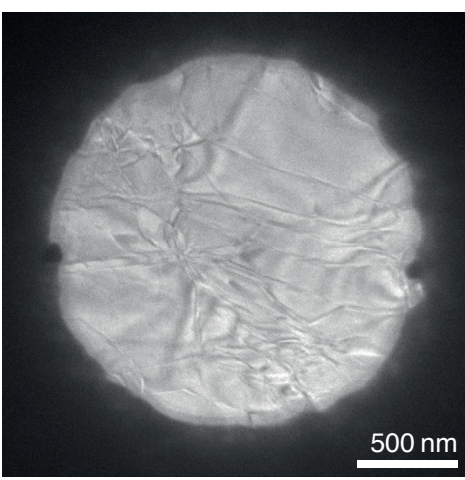

D

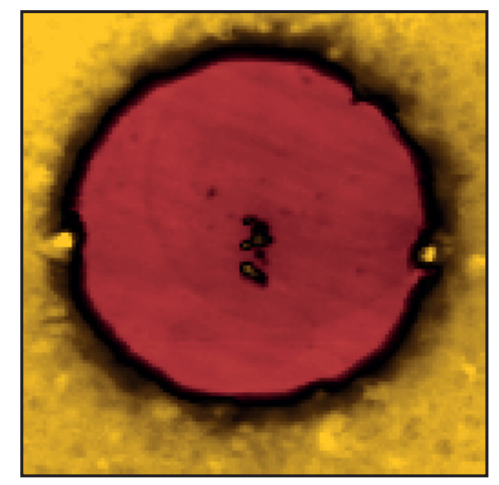

F
B

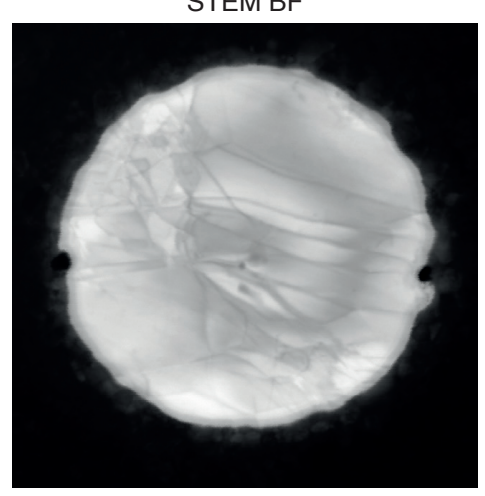

E

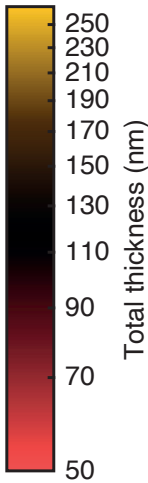

C

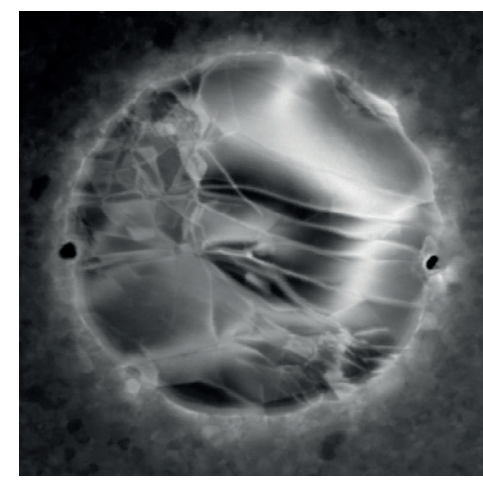

3D model of thickness map

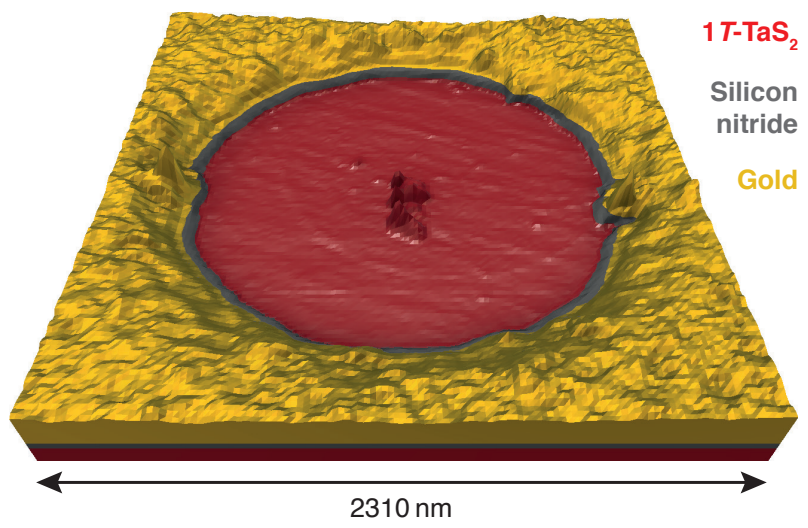

$2310 \mathrm{~nm}$

Cross section of simulation model

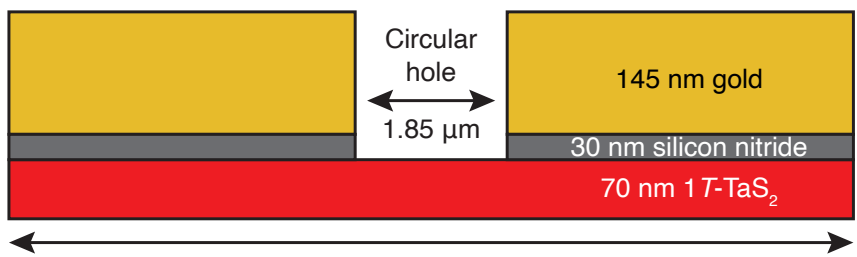

$10 \mu \mathrm{m}$ simulation box

Figure 5.9: Imaging of the specimen structure with a continuous electron beam (TEM imaging at $120 \mathrm{kV}$ acceleration voltage; STEM imaging at $200 \mathrm{kV}$ acceleration voltage).

(A) TEM image. (B) STEM bright-field (BF) image. (C) STEM annular DF image (i.e., using an annular electron detector). (D), (E) Specimen thickness map derived from STEM-EELS data. Different specimen components are indicated by different colors. (F) Cross-section of the simulation model used in the finite element simulations. 


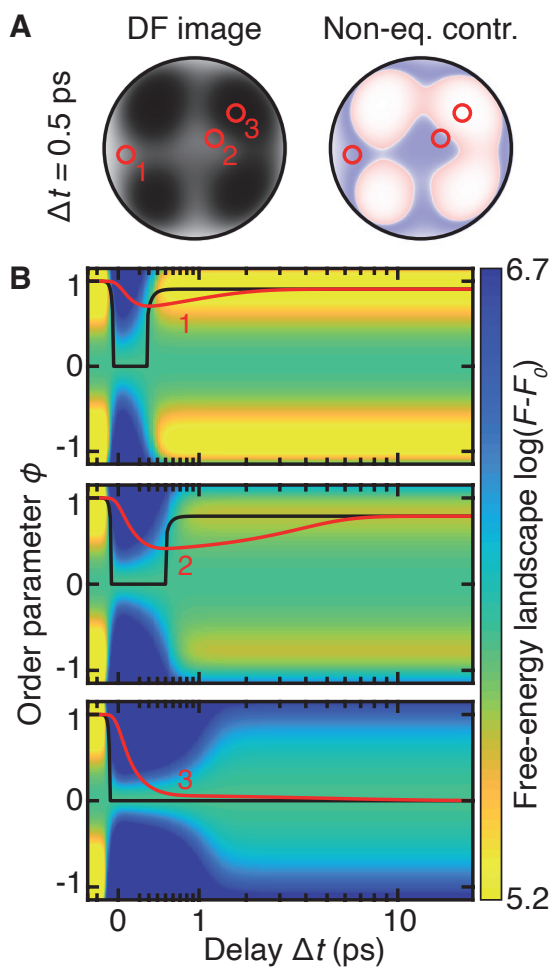

Figure 5.10: Order parameter trajectories on the free-energy surface for different levels of local excitation. (A) Representative locations with light NC CDW suppression (1), strong suppression (2), and IC phase formation (3) (cf. Fig. 5.4, A and C). (B) Order parameter trajectories at the locations indicated in (A). Red curve: Transient value of order parameter. Black line: Global free-energy minimum as a function of delay $\Delta t$ (shown for positive values of the order parameter only). 

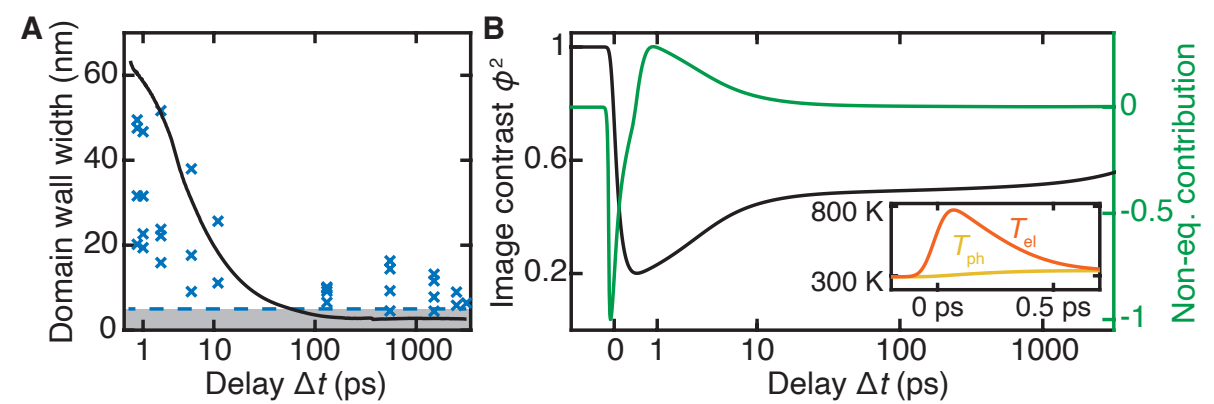

Figure 5.11: Domain wall width and extended simulation results.

(A) Width of NC/IC domain walls as extracted from time-resolved experimental data at different positions (blue data points; cf. Fig. 5.2A), and simulation results (black line; cf. Fig. 5.4A). The dashed blue line is the domain wall width extracted from the steady-state images (cf. Fig. 5.3A). The shaded region of the plot indicates the resolution limit of the experiment. (B) Spatially averaged delay curves corresponding to the simulation results in Fig. 5.4, A and C (black and green curves, respectively). Inset: Electron (orange) and lattice temperatures (yellow) during electron-lattice equilibration. 


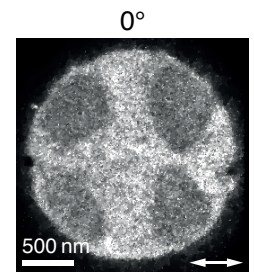

$25^{\circ}$

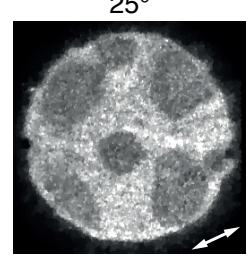

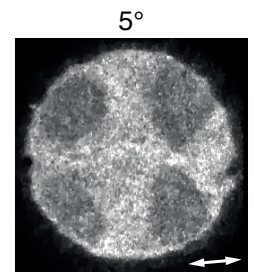

$30^{\circ}$

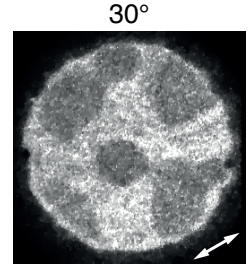

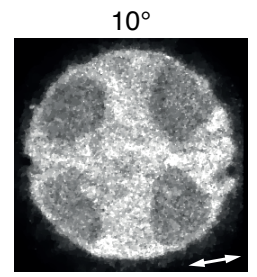

$35^{\circ}$

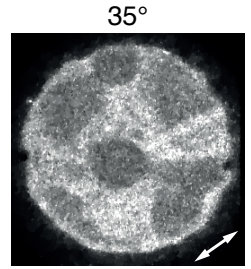

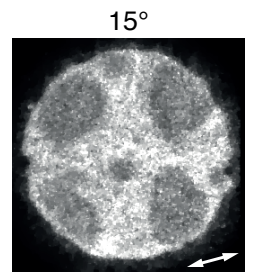

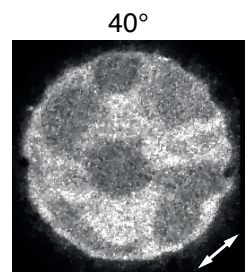

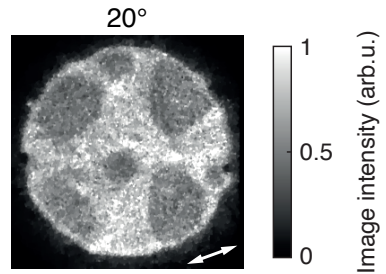

$45^{\circ}$

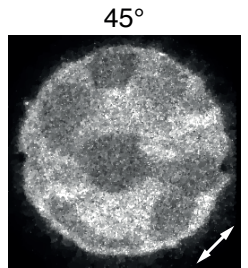

Figure 5.12: Ultrafast DF images as a function of linear pump polarization.

Pump polarization indicated by white arrows. Images taken at a fixed $550 \mathrm{ps}$ pump/probe delay and $2.6 \mathrm{~mJ} / \mathrm{cm}^{2}$ pump fluence (11 min integration time per frame). This figure is discussed in the supplementary text (see subsection "Structured excitation profile inside the circular gold aperture"). 

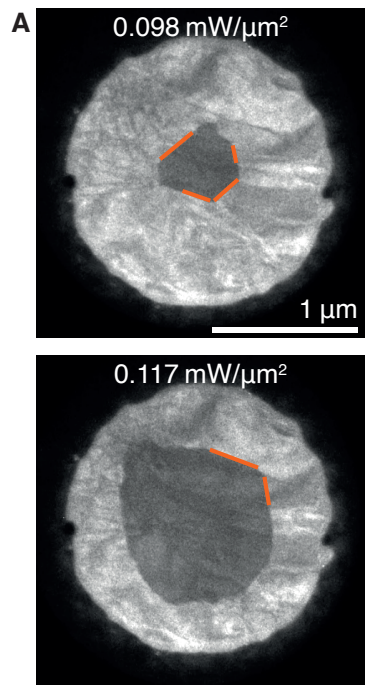
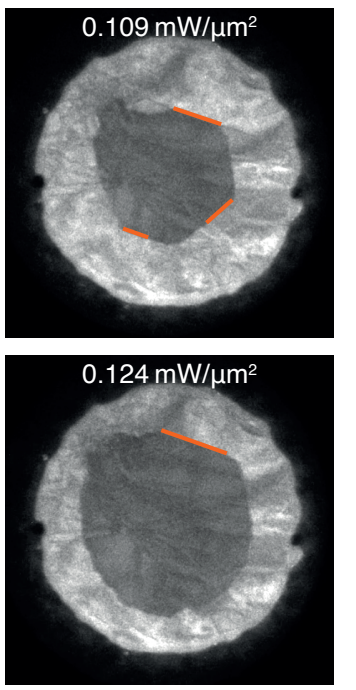
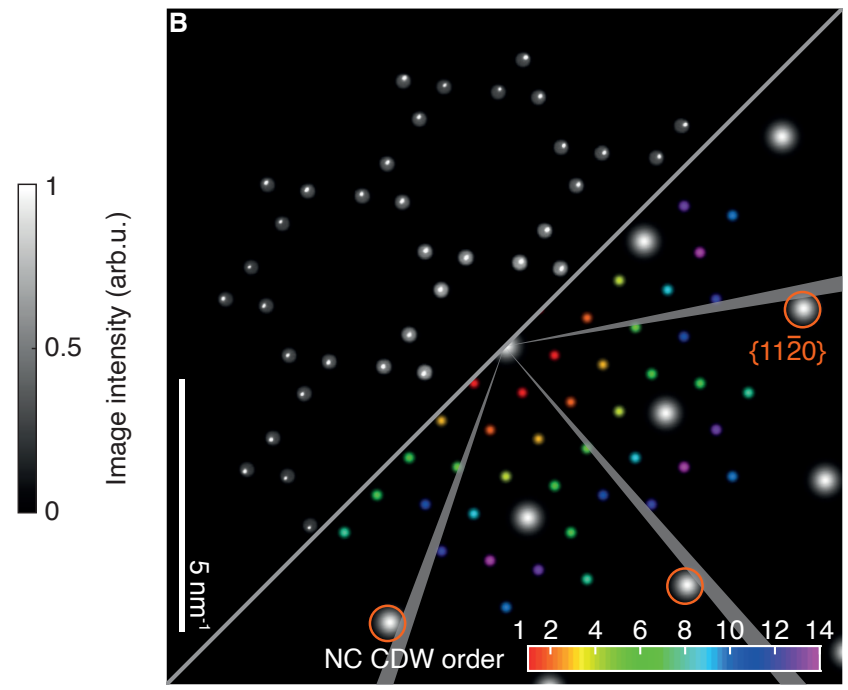

Figure 5.13: Identification of reciprocal lattice directions in the steady-state DF images.

(A) Selection of steady-state DF images that exhibit pronounced formation of NC/IC phase boundaries along preferential lattice directions. Orange lines indicate the subset of boundaries that has been used to determine the angular intervals shown in (B). (B) Top half: Diffraction image of the NC phase with the DF aperture array (only second-order satellites are visible). Bottom half: Diffraction pattern generated from theoretical spot positions of the underlying lattice (white spots) and of NC CDW orders from 1 to 14 that belong to the $\left(\begin{array}{llll}0 & 0 & 0 & 0\end{array}\right)$ structural reflection (rainbow color scale) (182). Grey angle intervals indicate the reciprocal lattice directions corresponding to the lattice planes indicated in (A). Both diffraction images have been aligned to precisely reflect the orientation of the real-space images. This figure is discussed in the supplementary text (see subsection "Orientation of NC/IC phase boundaries along preferential directions"). 


\subsection{Supplementary Tables}

\begin{tabular}{|c|c|c|c|}
\hline & Gold & $\begin{array}{l}\text { Silicon nitride } \\
\text { membrane }\end{array}$ & $1 T-\mathrm{TaS}_{2}$ \\
\hline $\begin{array}{l}\text { Complex refractive } \\
\text { index }\end{array}$ & $\begin{array}{l}0.53+2.22 i(532 \mathrm{~nm}) \\
0.15+4.91 i(800 \mathrm{~nm}) \\
(338)\end{array}$ & $\begin{array}{l}2.06(532 \mathrm{~nm}) \\
2.02(800 \mathrm{~nm}) \\
(339)\end{array}$ & $\begin{array}{l}2.25+1.46 i(532 \mathrm{~nm}) \\
3.01+2.09 i(800 \mathrm{~nm}) \\
(340)\end{array}$ \\
\hline $\begin{array}{l}\text { Density and molar } \\
\text { mass }\end{array}$ & $19.3 \mathrm{~g} / \mathrm{cm}^{3}(326)$ & $3.1 \mathrm{~g} / \mathrm{cm}^{3}(327)$ & $\begin{array}{l}6.86 \mathrm{~g} / \mathrm{cm}^{3} \\
245.078 \mathrm{~g} / \mathrm{mol}(326)\end{array}$ \\
\hline $\begin{array}{l}\text { Thermal } \\
\text { conductivity }\end{array}$ & $317 \mathrm{~W} /(\mathrm{m} \cdot \mathrm{K})(326)$ & $\begin{array}{l}\text { between } 3.66 \text { and } \\
6.08 \mathrm{~W} /(\mathrm{m} \cdot \mathrm{K}) \\
\text { (temperature range } \\
\text { from } 101 \text { to } 463 \mathrm{~K} \text { ) } \\
(341)\end{array}$ & $\begin{array}{l}\text { between } 3.82 \text { and } \\
8.38 \mathrm{~W} /(\mathrm{m} \cdot \mathrm{K}) \\
\text { electronic } \\
\text { contribution between } \\
0.00 \text { and } \\
2.68 \mathrm{~W} /(\mathrm{m} \cdot \mathrm{K}) \\
\text { lattice contribution } \\
\text { between } 3.61 \text { and } \\
5.81 \mathrm{~W} /(\mathrm{m} \cdot \mathrm{K}) \\
(\text { temperature range } \\
\text { from } 182 \text { to } 367 \mathrm{~K}) \\
(336)\end{array}$ \\
\hline $\begin{array}{l}\text { Specific heat } \\
\text { capacity }\end{array}$ & $129 \mathrm{~J} /(\mathrm{kg} \cdot \mathrm{K})(326)$ & $\begin{array}{l}\text { between } 241 \text { and } \\
919 \mathrm{~J} /(\mathrm{kg} \cdot \mathrm{K}) \\
\text { (temperature range } \\
\text { from } 101 \text { to } 457 \mathrm{~K}) \\
(341)\end{array}$ & $\begin{array}{l}\text { between } 12.2 \text { and } \\
54.1 \mathrm{cal} /(\mathrm{mol} \cdot \mathrm{K}) \\
\text { (temperature range } \\
\text { from } 172 \text { to } 367 \mathrm{~K}) \\
(335) \\
\text { linear electronic heat } \\
\text { capacity coefficient: } \\
8.5 \mathrm{~mJ} / \mathrm{mol} / \mathrm{K}^{2}(334)\end{array}$ \\
\hline
\end{tabular}

Table 5.1: Physical material properties used in the finite element simulations. 


\subsection{Supplementary Movies}

\section{Movie 5.1: Ultrafast DF image series.}

Data taken with $2.6 \mathrm{~mJ} / \mathrm{cm}^{2}$ pump fluence, $11 \mathrm{~min}$ integration time per frame, horizontal pump polarization. This dataset is used to extract the delay curves shown in Fig. 5.2C.

Movie 5.2: Ultrafast bright-field (BF) image series.

Data taken under the same experimental conditions as Movie 5.1 (except for a shorter 5.5 min integration time per frame). Only the DF aperture array is retracted from the column of the microscope. Note that no CDW contrast is visible, and that the movement of diffraction contrast lines indicates a mechanical oscillation of the membrane on nanosecond timescales, however, with no discernible influence on the DF images (cf. Movie 5.1).

Movie 5.3: Steady-state DF image series.

Data taken with $5 \mathrm{~s}$ integration time. A subset of these images is also presented in Fig. 5.3A.

Movie 5.4: Time-dependent Ginzburg-Landau simulation of ultrafast DF images.

A subset of these images is also presented in Fig. 5.4A.

Movie 5.5: Time-dependent Ginzburg-Landau simulation (non-equilibrium contribution). A subset of these images is also presented in Fig. 5.4C.

The movies are available as Supplementary Materials on the Science website at the following link: science.sciencemag.org/content/371/6527/371/suppl/DC1 (Movies S1 to S5). 


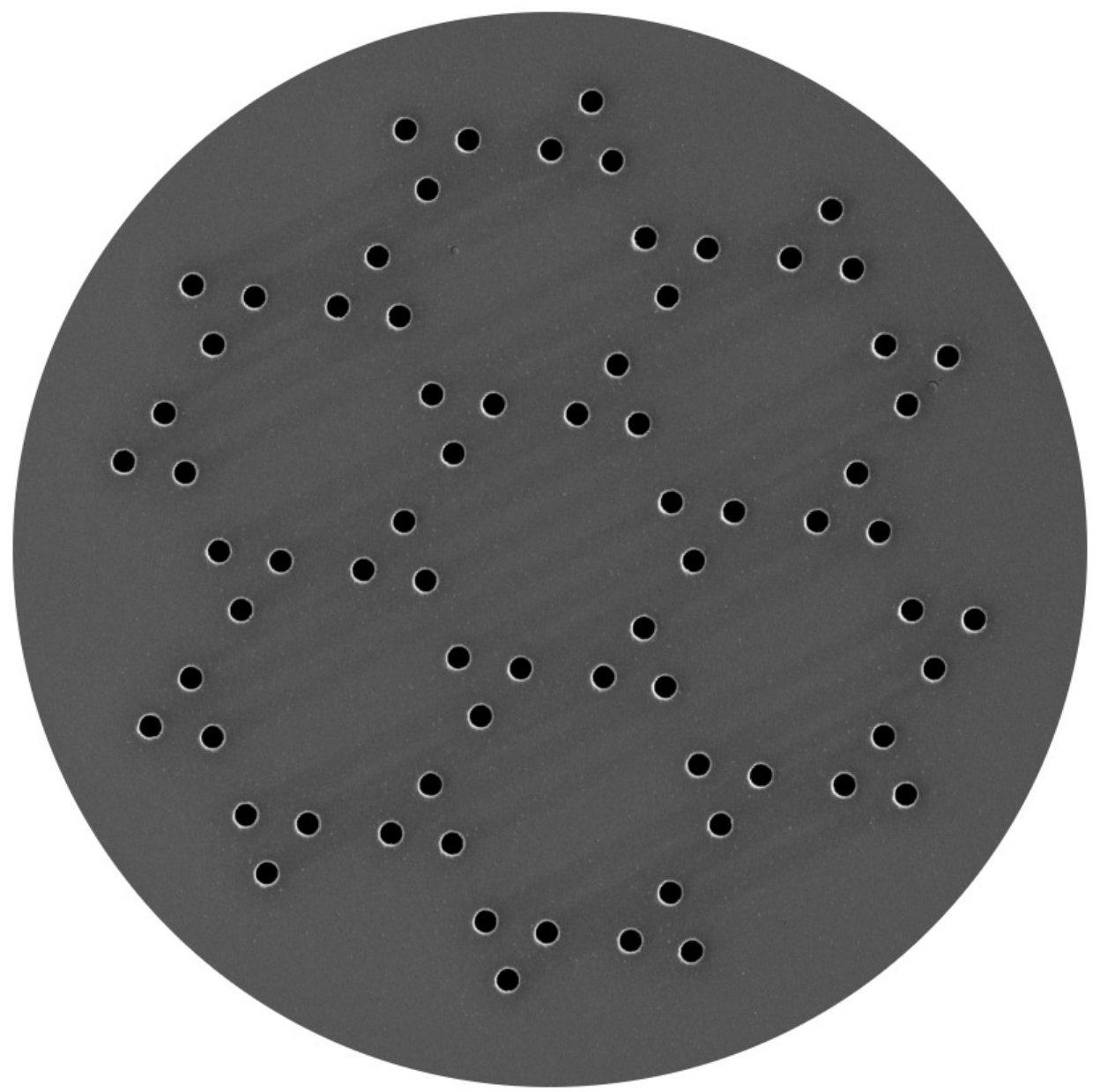

Tailored dark-field aperture array enabling sensitivity to the structural order parameter of the nearly commensurate charge-density wave phase in tantalum disulfide. See Figure 5.1 for more details. 



\section{Chapter 6}

\section{Discussion}

The work described in Chapters 4 and 5 of this thesis represents a major contribution to the rapidly evolving field of ultrafast electron microscopy and diffraction. The successful implementation of a novel nanoimaging approach at the Göttingen UTEM has been enabled by the following combination of distinct achievements and experimental capabilities:

- the use of ultramicrotomy to prepare large-area, free-standing films of layered TMDCs,

- the design of a specimen that spatially structures the optical excitation pattern and allows for stroboscopic probing of the NC/IC phase transition in $1 T-\mathrm{TaS}_{2}$ with hundreds of kilohertz repetition rates,

- the fabrication of a custom DF aperture array that is tailored to only transmit the intensities of 72 individual, second-order CDW reflections in the diffraction pattern of $1 T-\mathrm{TaS}_{2}$,

- the use of an in-plane rotation specimen holder that allows for aligning the specimen with the DF aperture array,

- the implementation of a direct electron detection camera at the Göttingen UTEM which allows for virtually background-free electron detection with a high DQE,

- and the high-coherence beam of the Göttingen UTEM, allowing for tightly focused diffraction spots in the BFP.

This chapter is structured in three parts: Firstly, we elaborate on the preparation and characterization of the specimen structure and give a broader perspective on the dichroic probing of magnetism in $\mathrm{Mn}_{0.25} \mathrm{TaS}_{2}$ and $\mathrm{Fe}_{0.25} \mathrm{TaS}_{2}$. Secondly, we discuss the points relating to the DF aperture array, the beam coherence and the reversibility of the CDW dynamics in $1 T-\mathrm{TaS}_{2}$. Additionally, we put our approach into context with other real-space imaging schemes and formulate perspectives for future ultrafast nanoimaging experiments with a focus on correlated materials. Finally, we briefly summarize the specific merits of the work presented in this thesis. 


\subsection{Preparation and characterization of thin films for UTEM experiments}

Chapter 4 describes a mechanical preparation technique for electron- and x-ray-transparent thin films of $\mathrm{Mn}_{0.25} \mathrm{TaS}_{2}$ and $\mathrm{Fe}_{0.25} \mathrm{TaS}_{2}$ using ultramicrotomy. This technique has then been applied to prepare $1 T-\mathrm{TaS}_{2}$ thin films for the ultrafast nanoimaging experiment described in Chapter 5 . Several static characterization methods were used to ensure high specimen quality and properties that do reflect those of the bulk crystals. Furthermore, we presented a specimen structure that allows for high-repetition-rate probing of the NC/IC phase transition in $1 T-\mathrm{TaS}_{2}$.

\subsubsection{Preparation of thin films using ultramicrotomy}

The investigated thin films of all three materials were cut using diamond knives with some differences in the specific procedures and ultramicrotome models. The specimens shown in Chapter 4 have been embedded in resin and sectioned using a "dEYEmond ULTRA $45^{\circ}$ " diamond knife and a "Leica EM UC6" ultramicrotome. The specimen in Chapter 5 has been cut directly from the bulk crystal which was glued to a block of gold using low-viscosity instant glue. Here, we used a predecessor-model ultramicrotome "Leica Ultracut UCT" with a "DiATOME Ultra $45^{\circ}$ " diamond knife. For our purposes, the only difference between both ultramicrotome models lies in comfort features implemented with the newer generation, such as a touch screen control panel instead of physical buttons, and optimized specimen illumination. Apart from that, no influence on the specimen quality, e.g., in terms of mechanical cutting precision was observed. Instead, environmental factors such as the amount of external vibrations present in the preparation room were found to have a drastic influence on the preparation.

Concerning the knives, there are only a handful of companies worldwide able to produce diamond knives for ultramicrotomy. This is due to the enormous experience necessary to sharpen a diamond knife to the atomic level. With the company "dEYEmond" having left the market, ultramicrotomy diamond knives are sold by the Swiss market leader "DiATOME" and a few other vendors. While the knife used to prepare the thin films of the intercalated specimens exhibited some knife edge wear due to prior use, similar damage appeared on the DiATOME knife edge after an extended period of use. Therefore, we are unable to identify a difference in the quality of both knives.

Specimen preparation by ultramicrotomy has been popularized in the material sciences in the 1990s (106). While, nowadays, some of the earlier advantages over competing preparation methods are not entirely valid anymore, ultramicrotomy is still able to deliver large-area, highquality thin films whose atomic and electronic structure is unmodified with respect to the 
bulk. Most importantly, and in contrast to the widely used FIB preparation of TEM specimens, there is no formation of an amorphous surface layer and no implantation of impurity atoms (342). Additionally, no other method than ultramicrotomy can deliver large-area films of the $3 d$ ion-intercalated TMDCs $\mathrm{Mn}_{0.25} \mathrm{TaS}_{2}$ and $\mathrm{Fe}_{0.25} \mathrm{TaS}_{2}$, while competing with the mechanical exfoliation technique in case of $1 T-\mathrm{TaS}_{2}$ (105). However, the specimen thickness of exfoliated films can only be post-characterized using, e.g., atomic force microscopy (AFM) or TEM. Still, these films can exhibit very homogeneous thickness distributions, while ultramicrotomed films may suffer from some degree of striation due to knife edge wear (cf. Fig. 4.1C).

In time-resolved electron diffraction experiments without spatial resolution, large and homogeneous specimen areas are advantageous. For the case of TMDCs, this can be obtained in the reflection geometry by in situ cleaving of a bulk crystal (250). In a transmission geometry, ultramicrotomed thin films can be used analogously. For the case of $1 T-\mathrm{TaS}_{2}$, this has first been demonstrated by EICHBERgER et al. in an UED experiment (65). Other UED experiments using ultramicrotomed films are described in Refs. $(265,266)$.

EICHBERGER et al. published a follow-up article in which the preparation procedure for the $30 \mathrm{~nm} 1 T-\mathrm{TaS}_{2}$ films is described in more detail (302). In this reference, a reaction of the tantalum atoms in the material with the diamond knife was suggested, leading to a rapid deterioration of the knife edge. After some more years of experience, we can confirm the early observation from section 4.4.1 that we are unable to identify a specific reaction of this kind. In contrast, we attribute the damage of our DiATOME knife to the usual wear and tear. However, the expectation of tantalum-induced effects is certainly not wrong as there are, in fact, observations of the formation of a tantalum carbide layer when evaporating tantalum on diamond (343).

\subsubsection{Specimen thickness measurements}

In the present thesis, EELS-based thickness measurements (section 2.5) have been used to assess the thickness of thin films prepared by ultramicrotomy. For most UTEM experiments, specimen thicknesses in the range of $50 \mathrm{~nm}$ to $100 \mathrm{~nm}$ are desirable. Although the thickness is generally controlled by adjusting the feed rate of the ultramicrotome, a faulty calibration of the feed rate or vibrations during the sectioning process can lead to deviations in the actual thickness. In Chapter 4, the MFP of $\mathrm{Mn}_{0.25} \mathrm{TaS}_{2}$ was calculated using the model by MALIs et al. (Eq. 2.9) in order to derive the thickness of the specimen shown in Fig. 4.1C from EELS spectra. While the specimen has a nominal thickness of $100 \mathrm{~nm}$, the EELS measurements resulted in values between $80 \mathrm{~nm}$ and $100 \mathrm{~nm}$. 
The MFP of the individual components in the $1 T-\mathrm{TaS}_{2} /$ silicon nitride/gold specimen structure reported in Chapter 5 has been derived according to IАкоuвovski et al. (Eq. 2.12). A comparison of these values to values obtained for the same materials using the model by MaLis et al. is shown in Table 6.1. It becomes apparent that Eq. 2.9 probably underestimates the MFP and that the exact choice of $Z$ is of main significance for compounds containing both heavy and light elements (77). We determined an average $1 T-\mathrm{TaS}_{2}$ thickness of $70 \mathrm{~nm}$ based on the MFP of $126 \mathrm{~nm}$, while a feed rate of $50 \mathrm{~nm}$ was set at the ultramicrotome during preparation (see Fig. 5.9D and E for thickness maps).

\begin{tabular}{|l|c|c|c|}
\hline MFP model / Material & Gold & $\begin{array}{c}\text { Silicon nitride } \\
\text { membrane }\end{array}$ & $\mathbf{1 T}$-TaS \\
\hline MALIS et al. (based on $\bar{Z}$ ) & $71 \mathrm{~nm}$ & $130 \mathrm{~nm}$ & $90 \mathrm{~nm}$ \\
\hline MALIS et al. (based on $Z_{\text {eff }}$ ) & $71 \mathrm{~nm}$ & $128 \mathrm{~nm}$ & $86 \mathrm{~nm}$ \\
\hline IAKOUBOvSKII et al. (based on $\rho$ ) & $99 \mathrm{~nm}$ & $153 \mathrm{~nm}$ & $126 \mathrm{~nm}$ \\
\hline
\end{tabular}

Table 6.1: Comparison of calculated MFP values for gold, silicon nitride and $\mathbf{1 T}$-TaS $\mathbf{S}_{\mathbf{2}}$ The values have been derived using the models given in Eqs. 2.9 (Malis et al.) and 2.12 (IAкоubovsKi et al.) for the experimental conditions reported in section 5.2.3.

\subsubsection{Further specimen characterization}

Besides direct thickness measurements, additional specimen characterization techniques can be helpful to assess the quality of ultramicrotomed thin films. In Chapter 4, we demonstrated a specimen characterization approach using light microscopy, TEM diffraction and static XMCD at a synchrotron beamline.

Light microscopy in reflected-light mode is very well suited to assess the overall morphology of a thin film as demonstrated in Fig. 4.1C and D. Although no transmitted-light images have been taken from the intercalated TMDC specimens, the transmitted-light image of an ultramicrotomed graphite flake in Fig. 2.5B demonstrates that this contrast reveals the degree of homogeneneity of the specimen thickness on a macroscopic scale.

TEM diffraction can, as outlined in section 3.7, be used to probe both superlattices: those due to intercalant long-range ordering and those due to the presence of a PLD. For the case of the $\mathrm{Mn}_{0.25} \mathrm{TaS}_{2}$ and $\mathrm{Fe}_{0.25} \mathrm{TaS}_{2}$ thin films, we could verify the structural integrity after ultramicrotome preparation by the presence of the $2 \times 2$ supercell formation. In $1 T-\mathrm{TaS}_{2}$, the occurrence of the NC CDW in room-temperature diffraction patterns indicates intact electronic and structural properties. Most importantly, the diffraction patterns only exhibit strong superstructure reflections of the second order and weaker sixth-order spots (Fig. 5.1C, top), as is dictated 
by the three-fold CDW stacking periodicity (section 3.4). Equally, we only see second-order superstructure reflections in the laser-heated IC phase (Fig. 5.5). For the case of $\mathrm{Mn}_{0.25} \mathrm{TaS}_{2}$ and $\mathrm{Fe}_{0.25} \mathrm{TaS}_{2}, \mathrm{XMCD}$ delivers valuable insights into the thin film integrity by measuring the magnetic properties of the material. Hysteresis curves at an absorption edge of the intercalant ion can be used to verify if the Curie temperature and coercivity of the material are unchanged with respect to the bulk material (Fig. 4.4).

The thin films of $\mathrm{Mn}_{0.25} \mathrm{TaS}_{2}$ and $\mathrm{Fe}_{0.25} \mathrm{TaS}_{2}$ presented in Chapter 4 are a considerable advantage for XMCD experiments. With bulk specimens, the acquisition of XMCD spectra is commonly done using TEY and FY techniques. The concept behind TEY and FY is that, after absorption of the X-ray beam, the number of emitted photoelectrons or photons is proportional to the absorption coefficient. However, some effects lead to a deviation from the linear relationship. For the case of TEY measurements, saturation effects in the photoelectron response occur as a function of x-ray photon energy when the x-ray attenuation length approaches the electron escape depth (298). In FY measurements, self-absorption effects lead to a strong dependency of the overall shape of the spectra on the detection geometry (344). Using ultramicrotomed thin films, the transmission method allows for the most direct access to quantitative data, as demonstrated in Ref. (345) and for the ratio of orbital to spin magnetic moment (section 4.4.3).

\subsubsection{A broader perspective on the dichroic probing of magnetism}

$\mathrm{XMCD}$ is a complementary technique to UTEM in the sense that it is sensitive to the out-of-plane component of the magnetization only. In TEMs, Lorentz and differential phase contrast imaging can be used to reconstruct the in-plane magnetization component on the nanoscale $(76,151$, 346), but without element specificity. Sensitivity to the other component can be obtained by tilting the specimen away from perpendicular incidence of the x-ray or electron beam. Both approaches can be brought into the time domain, as demonstrated in the past for ultrafast Lorentz imaging (102-104, 310) and ultrafast XMCD at synchrotron light sources and free-electron lasers $(290,347)$. Using $\mathrm{X}$-ray holography or coherent diffractive imaging (CDI) techniques, the XMCD method can be combined with few-10 nm spatial magnetic imaging resolution $(347,348)$. Newer developments even significantly expand the applicability of dichroic imaging to table-top high-harmonic generation setups (349). Recently, a combined spatiotemporal resolution of $16 \mathrm{~nm}$ and $40 \mathrm{fs}$ in an ultrafast demagnetization experiment has been reported (350).

Additionally, there is an electron-beam equivalent to XMCD that was experimentally confirmed by Schattschneider et al. only in 2006 (351). Electron energy-loss magnetic chiral dichroism 

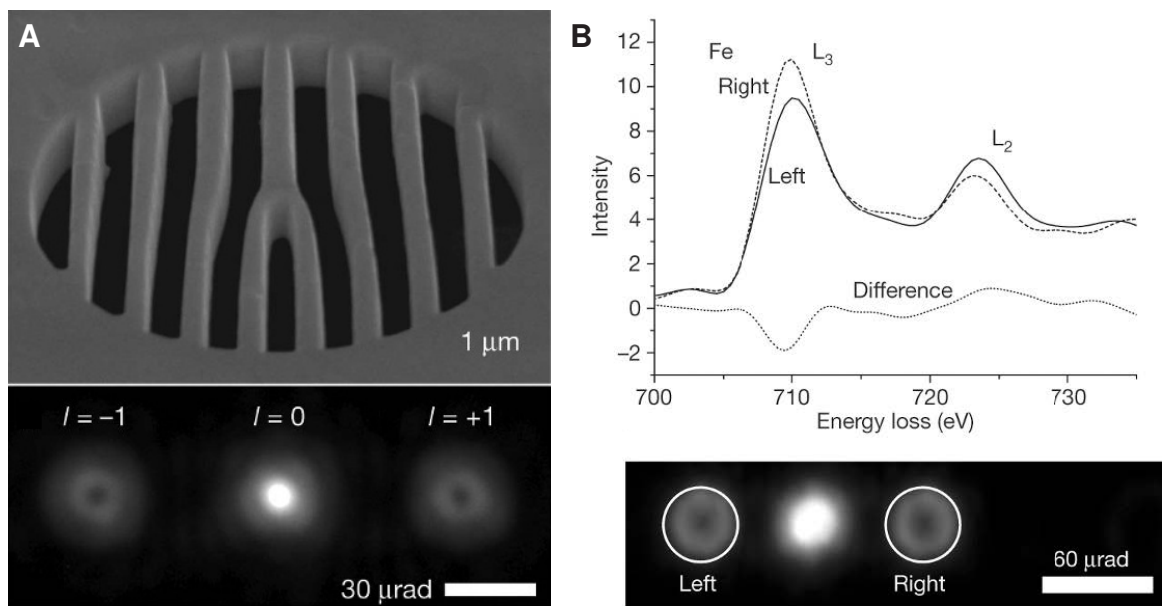

Figure 6.1: Application of electron vortex beams to EMCD. (A) A binary mask with an edge dislocation can be used to generate electron beams with non-zero orbital angular momentum (top). Beams with a topological charge of $l=0$ and $l= \pm 1$ are observed in the far-field diffraction pattern (bottom). (B) In order to record EMCD spectra of the iron $L_{3,2}$ absorption edges (top), the mask is inserted into the electron beam path below the specimen plane. Using EELS, dichroic spectra can be extracted from the intensity of the left and right sidebands with $l= \pm 1$ (bottom). Reprinted by permission from Springer Nature Customer Service Centre GmbH: Springer Nature, Nature, Ref. (320), Copyright 2010 Nature Publishing Group, a division of Macmillan Publishers Limited.

(EMCD) has since been successfully applied to measure magnetic properties of different materials in the TEM $(352,353)$. Later on, it has also been shown that electron vortex beams carrying orbital angular momentum $l \hbar$ with a topological charge of $l= \pm 1$ can be used to obtain a higher SNR in comparison to the standard method (Fig. 6.1) (320). Still, the use of vortex beams for EMCD is impeded by a number of experimental complications $(354,355)$.

An alternate route to measuring magnetization using electrons are spin-polarized electron beams. Employing the spin-dependent reflectivity of a ferromagnetic surface, magnetic domain structures can be imaged using spin-polarized low-energy electron microscopy (SPLEEM) (36, $356,357)$. When incorporated in a scanning electron microscope with polarization analysis (SEMPA), a SPLEEM-based detector can even be used to detect the spin polarization of secondary electrons originating from a magnetic specimen (358). On the UTEM side, the implementation of a picosecond-pulsed spin-polarized electron source has also been realized in the past (132).

Although the signal in the high-loss regime is expected to be rather faint, future technological advancements will almost certainly open up the possibility of conducting time-resolved EMCD measurements using UTEM and offer intriguing experimental possibilities. Concerning the two materials $\mathrm{Mn}_{0.25} \mathrm{TaS}_{2}$ and $\mathrm{Fe}_{0.25} \mathrm{TaS}_{2}$, both are understood to be RKKY ferromagnets which leads to some spin polarization in the $5 d_{z^{2}}$ bands of tantalum $(286,299)$. However, in $\mathrm{Mn}_{0.25} \mathrm{TaS}_{2}$, a strong hybridization between the tantalum $5 d_{z^{2}}$ and manganese $3 d$ states occurs, leading to a 
delocalization of the manganese electrons and to the appearance of itinerant magnetism (299). Therefore, it would certainly be interesting to probe the respective magnetic moments on the tantalum atoms in the two materials at the tantalum $M_{5,4}$ edge at loss energies of $1735 \mathrm{keV}$ and $1793 \mathrm{keV}$ (359), both statically and time-resolved.

\subsubsection{Design of the specimen structure for the DF experiment}

In contrast to reflection-geometry experiments, where the bulk of the specimen can absorb a great amount of laser-deposited heat, thermal conductivity in free-standing thin films has a two-dimensional character due to the extremely high aspect ratio (the film shown in Fig. 4.1C has an aspect ratio of approximately 4000:1), and the total heat capacity of the specimen is much smaller. Therefore, transmission-type experiments are typically conducted at repetition rates between $\sim 100 \mathrm{~Hz}(24,237)$ and a few $\mathrm{kHz}(58,65,360)$.

One of the specific merits of the real-space imaging experiment described in Chapter 5 is the stroboscopic probing of a $70 \mathrm{~nm} 1 T-\mathrm{TaS}_{2}$ thin film at hundreds of $\mathrm{kHz}$ repetition rates. While the presented data has been acquired at a $420 \mathrm{kHz}$ repetition rate, we have also been able to drive the NC/IC phase transition in the same specimen structure at a $600 \mathrm{kHz}$ repetition rate. This was, at that point in time, only limited by the maximum stable repetition rate of the "Coherent RegA" laser. The ultrafast DF images in Fig. 5.2A reveal that the last IC domains relax back into the NC phase after slightly more than $3 \mathrm{~ns}$, such that the specimen must have cooled down well below the threshold after $100 \mathrm{~ns}$ (corresponding to $10 \mathrm{MHz}$ ). This makes room for the assumption that it should even be possible to reversibly drive the phase transition at few-MHz repetition rates.

Instrumental to this success is the design of the specimen structure as shown in Figs. 5.1A and 5.9F. In the experiment, the laser beam is focused down to $15 \mu \mathrm{m}$ FWHM diameter, while the diffraction limited focus diameter should be on the order of $5 \mu \mathrm{m}$ to $10 \mu \mathrm{m}$. The deviation between those values is probably due to aberrations collected in the focusing lens and due to some clipping along the optical incoupling port of the UTEM. If a homogeneous specimen had been excited using a $15 \mu \mathrm{m}$ beam, the absorbed energy would be approximately 65 times larger than the energy deposited within the $1.85 \mu \mathrm{m}$ circular aperture of the specimen structure. Furthermore, the presence of the gold film around the aperture ensures coupling to a macroscopic heat bath. The good thermal coupling and the confinement of the excitation to the experimental region of interest are two key components of the design of the specimen structure.

Additionally, the diameter and depth of the circular aperture are on the order of the pump wavelength, giving rise to the onset of waveguiding effects. This results in a complex optical 
mode profile at the specimen surface which determines the inhomogeneous shape of the excitation density shown in Fig. 5.2E. The consequential strong thermal gradients imprinted on the specimen at early times enable the observed spatiotemporal domain evolution in the first place (Fig. 5.2A). Despite a slight distortion caused by the irregular edges of the aperture, the observed four-lobe pattern is expected due to the symmetry breaking by the linear optical polarization (section 5.3.1). For more regular shapes of the aperture, numerical simulations (section 5.2.8) can be used perspectively to predict and tailor the optical mode profile at the specimen surface.

Although one of the strengths of UTEM is its ability to locally probe inhomogeneous systems, the use of ultramicrotomed $1 T-\mathrm{TaS}_{2}$ films still is a great advantage in the preparation process (section 5.2.3). After coating the backside of the silicon nitride membrane with the gold reflection layer and milling the circular aperture using FIB, the large area of the ultramicrotomed $1 T-\mathrm{TaS}_{2}$ film allows for some degree of freedom in its subsequent placement on top of the aperture.

\subsubsection{Alternate routes to high-repetition-rate probing}

For future experiments, a FIB-based transfer process as shown in Fig. 6.2 may be used as an alternative to the manual film placement. In this case, a small $1 T-\mathrm{TaS}_{2}$ flake had been transferred onto a standard TEM copper grid after ultramicrotome preparation. A micromanipulator was then used to extract one mesh cell, transfer it onto a silicon nitride membrane and mount it using FIB-deposited platinum. In analogy to the preparation of the DF specimen structure, the membrane had a front-side gold coating and a circular through-hole prepared in advance.

However, static TEM diffraction after preparation of the FIB-based structure has only revealed the hexagonal lattice of the material, and no trace of CDW superstructure reflections. It has to be assumed that implantation of scattered gallium ions during the preparation process severely damaged the electronic structure of the material (342), effectively suppressing CDW formation. This indicates that even more care has to be taken in future FIB-based approaches not to contaminate the $1 T-\mathrm{TaS}_{2}$ film. The same problem has also been reported to occur when using reactive ion etching processes for the preparation of $1 T-\mathrm{TaS}_{2}$ thin films. While energy-dispersive $\mathrm{x}$-ray spectroscopy reveals almost perfect stoichiometry for ultramicrotomed samples, reactiveion-etched samples exhibit large fractions of oxygen and carbon (302).

Generally, the combination of pulsed laser excitation and FIB-deposited platinum is problematic. The resulting amorphous platinum compound contains a large fraction of organic byproducts of the gaseous precursor. For platinum deposition, the organometallic precursor trimethyl(methylcyclopentadienyl)platinum $\left(\mathrm{MeCpPtMe}_{3}\right)$ is a common choice. Some of the 

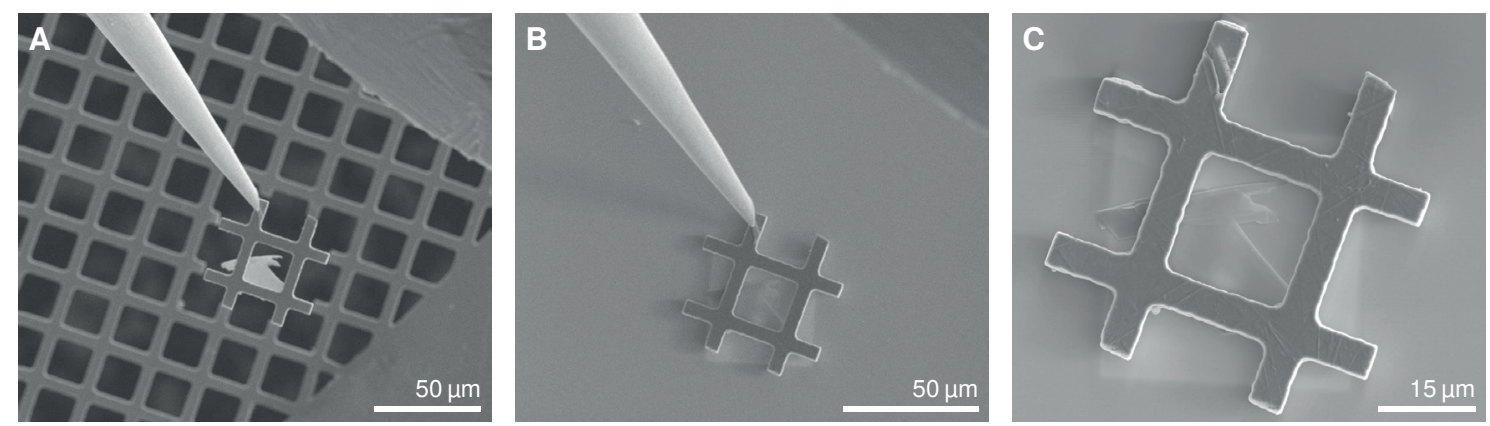

Figure 6.2: Transfer of a $1 T-\mathrm{TaS}_{2}$ film using FIB manipulation. (A) A micromanipulator is secured to the copper grid using ion-beam-assisted platinum deposition. The grid bars are cut by FIB milling before lifting the extracted portion of the grid. (B) Subsequently, the grid portion is positioned on a gold-coated silicon nitride membrane and fixed using platinum deposition. (C) In a last step, the micromanipulator is detached using FIB milling. A through-hole in the membrane (below the $1 T-\mathrm{TaS}_{2}$ flake) prepared in advance defines the region of interest for TEM.
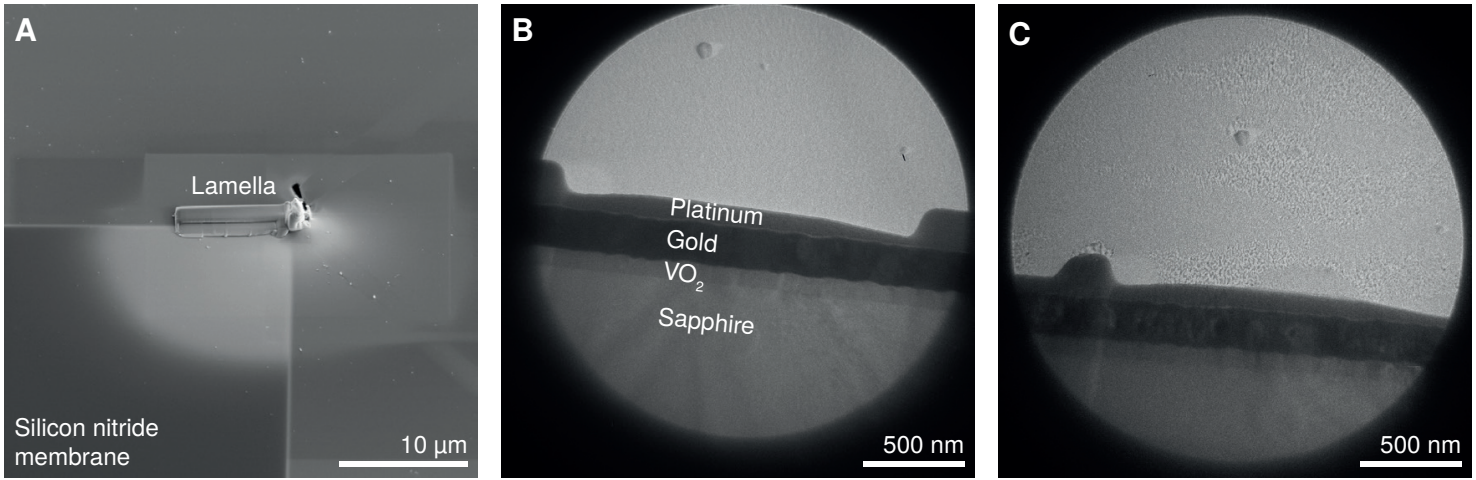

Figure 6.3: Crystallization of FIB-deposited platinum. (A) Scanning electron micrograph of a cross-sectional $\mathrm{VO}_{2}$ lamella after placement on a pristine silicon nitride membrane (dark area in the bottom left corner). Some platinum residue is visible on the membrane (white quarter circle). (B) TEM image of the lamella with the different layers indicated. (C) After exposure to ultrashort laser pulses, platinum crystallization is visible on the silicon nitride membrane. 
precursor is also deposited in undesired regions of the specimen. Under laser illumination, these leftovers crystallize and modify the surface, changing for example its optical properties. This is shown in Fig. 6.3 using the example of a $\mathrm{VO}_{2}$ lamella. The effect of this kind of laser annealing is probably very similar to thermal annealing as a post-deposition treatment (361).

As an alternative, stroboscopic repetition rates could be increased by optimizing the thermal conductivity in the experimental region of interest. While the specimen structure used in the nanoimaging experiment features a free-standing film of $1 T-\mathrm{TaS}_{2}$, the use of a sufficiently electron-transparent substrate with a low scattering cross-section and high thermal conductivity may help to reach that goal. Standard choices for TEM are amorphous carbon and silicon nitride membranes, but there are also more uncommon options such as single-crystalline silicon and even diamond membranes.

\begin{tabular}{|l|cc|}
\hline Material & Thermal conductivity & References \\
\hline Silicon nitride membrane (amorphous) & $4.9 \mathrm{~W} /(\mathrm{m} \cdot \mathrm{K})$ & $(341)$ \\
\hline Silicon (bulk) & $148 \mathrm{~W} /(\mathrm{m} \cdot \mathrm{K})$ & $(326)$ \\
\hline Silicon membrane (single-crystalline) & $22 \mathrm{~W} /(\mathrm{m} \cdot \mathrm{K})$ & $(362)$ \\
\hline Carbon membrane (amorphous) & 0.2 to $2.2 \mathrm{~W} /(\mathrm{m} \cdot \mathrm{K})$ & $(363)$ \\
\hline IIa diamond (bulk) & $2300 \mathrm{~W} /(\mathrm{m} \cdot \mathrm{K})$ & $(326)$ \\
\hline CVD diamond (bulk, polycrystalline) & $>1200 \mathrm{~W} /(\mathrm{m} \cdot \mathrm{K})$ & $(364)$ \\
\hline Diamond membrane (polycrystalline) & $\begin{array}{c}\text { Reduced by a factor } \\
\text { of } 5 \text { to } 8 \text { w.r.t. IIa diamond }\end{array}$ & $(365)$ \\
\hline
\end{tabular}

Table 6.2: Thermal conductivities of TEM membranes (in-plane) and bulk materials. All values are given at or close to room temperature.

As shown in Table 6.2, these materials differ widely in their thermal conductivities. The obvious choice from this point of view is diamond due to its extremely high thermal conductivity. Polycrystalline diamond membranes with thicknesses of a few tens of nanometers can be produced by chemical vapor deposition (CVD) and a following etching process. While bulk CVD diamond reaches at least half of the thermal conductivity of IIa diamond (natural diamond that contains the lowest density of impurities), the value is found to be reduced in polycrystalline membranes by a factor of 5 to 8 with respect to IIa diamond (365). With that, the thermal conductivity would still be a factor of two higher than that of bulk silicon (326), and more than one order of magnitude higher than that of single-crystalline silicon membranes (362).

To test the usefulness of diamond substrates in UTEM experiments, we obtained membranes from two companies, "Diamond Materials" and "Applied Diamond". The respective membrane thicknesses are 50 and $110 \mathrm{~nm}$, and $40 \mathrm{~nm}$. Figure 6.4A shows a TEM image of one of the diamond membranes. In the out-of-focus image, one can see the diamond grains whose different 

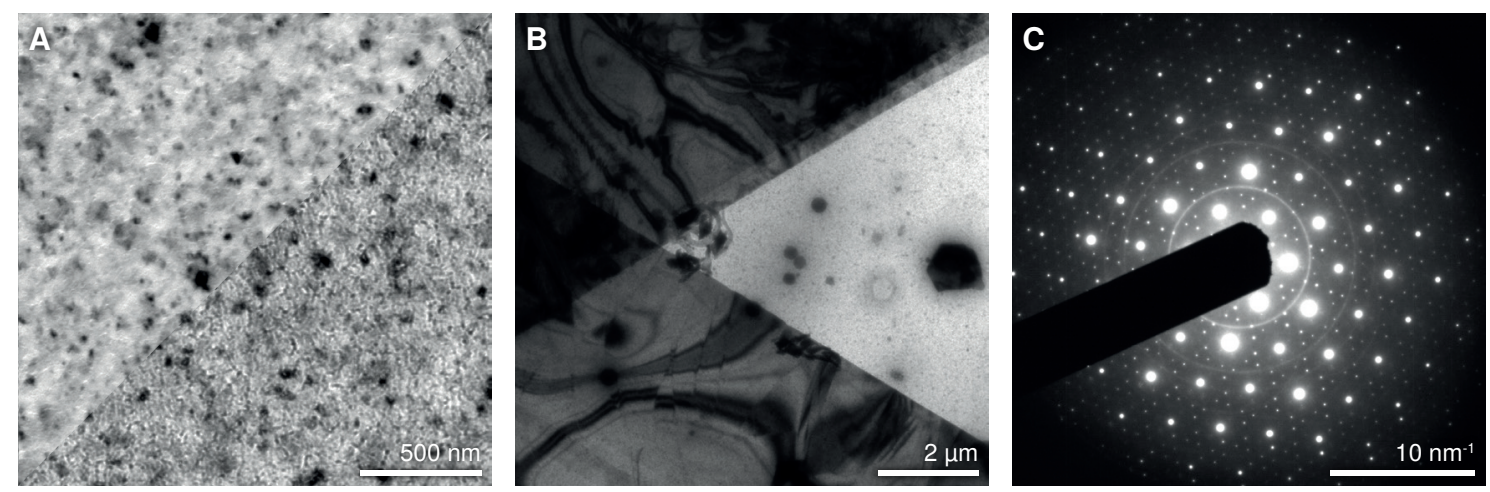

Figure 6.4: TEM images and diffraction patterns of polycrystalline diamond membranes. (A) TEM image of a $50 \mathrm{~nm}$ diamond membrane (Diamond Materials) imaged under in-focus (top half) and out-of-focus (bottom half) conditions. The out-of-focus image visualizes the nanoscale grain boundaries in the film. (B) TEM image of a $40 \mathrm{~nm}$ diamond membrane (Applied Diamond) with two overlapping ultramicrotomed $1 T-\mathrm{TaS}_{2}$ flakes. (C) Diffraction image taken from the upper flake in B. The polycrystalline diamond membrane gives rise to diffraction rings in addition to the structural and CDW reflections of $1 T-\mathrm{TaS}_{2}$.

orientations lead to the granular contrast in the in-focus image. Generally, there is no large difference to other membrane materials in terms of handling, and $1 T-\mathrm{TaS}_{2}$ flakes obtained by ultramicrotomy can be deposited on the membranes for TEM observation (Fig. 6.4B). In the diffraction images, strong scattering from the diamond grains overlaps with the hexagonal pattern of $1 T-\mathrm{TaS}_{2}$ (Fig. 6.4C).

Further inspection of diffraction patterns obtained from empty diamond membranes reveals that a large fraction of the electron beam is elastically scattered by the membrane alone. We estimate that $83 \%$ and $38 \%$ of the electrons remain unscattered while passing through the $50 \mathrm{~nm}$ and $110 \mathrm{~nm}$ membranes, respectively. The two $40 \mathrm{~nm}$ membranes by the second manufacturer left $63 \%$ and $77 \%$ of the incident electrons unscattered. This large scattering cross-section leads to multiple scattering effects that are evident from additional diffraction rings centered around the brightest structural reflections of $1 T-\mathrm{TaS}_{2}$.

We find that the gain in thermal coupling of the specimen is not high enough to tolerate the pronounced elastic scattering background. While heating intensities on the order of $0.15 \mathrm{~mW} / \mu \mathrm{m}^{2}$ were necessary to statically heat most of the specimen structure discussed in section 6.1.5 to temperatures above the NC/IC phase transition temperature (Fig. 5.3A), we experimentally found that values about 100 times smaller are necessary to reach the same local temperature in the specimen shown in Fig. 6.4B. In conclusion, it is much more efficient to confine the specimen excitation to the region of interest of the UTEM experiment than to improve the thermal coupling by choice of substrate only. 


\subsection{Ultrafast nanoimaging using UTEM}

In Chapter 5, we describe the application of the tailored DF aperture array for ultrafast and static imaging of CDW domain formation in $1 T-\mathrm{TaS}_{2}$. In the following sections, we will discuss details of the nanoimaging approach going beyond the scope of the Supplementary Materials of the original publication (sections 5.2 to 5.6).

\subsubsection{Feasibility of the DF imaging technique}

As outlined at the beginning of this chapter, the ultrafast nanoimaging experiment is a first of its kind due to the combination of a number of unique components and concepts. These deal in part with the low electron dose and the consequential long integration times affecting all ultrafast electron imaging and diffraction experiments in the single-electron regime.

The DF aperture most certainly is the central component of those. Except for the occasional use of annular DF apertures (366-369) and different types of phase plates $(95,322,370,371)$ for contrast enhancement and reduction of aberrations, there are no reports concerning a similarly complex DF aperture to be found in the literature. This can be attributed to the relatively small added value for static TEM techniques compared to the resultant loss of experimental flexibility. Noteworthy, in their 1995 paper, Ishiguro and SATo describe the use of a bandpass filter very similar to our DF aperture array in the post-processing of static high-resolution TEM images of the NC phase of $1 T-\mathrm{TaS}_{2}$ (372). However, by filtering at the time of acquisition, we are able to image nanoscale domain formation without the need to resolve individual atomic positions.

The main challenge of ultrafast DF imaging of CDW dynamics is the vanishingly small fraction of electrons scattered by the superstructure periodicities. For the case of the diffraction image shown in the top panel of Fig. 5.1C, the total intensity in those 72 superstructure reflections that are transmitted through the DF aperture array is (after background subtraction) approximately $0.01 \%$ of the total scattered intensity. Considering the fact that the integration time needed to acquire each of the DF images shown in Fig. 5.2A amounts to $\sim 1 \mathrm{~h}$, it becomes very clear that the nanoimaging experiment would not be feasible with the use of an individual circular aperture. With the smallest conventional objective aperture typically available in TEMs (5 $\mu \mathrm{m}$ diameter), the inelastic background impairing the DF image contrast would be higher by a factor of $\sim 8$ - and integration times would be 72 times longer as well.

The aperture design could be even further optimized by including additional superstructure reflections. The present design considers all second-order CDW spots that are arranged around 
the direct beam and the first-order Bragg spots. However, there is still considerable intensity contained in the 72 additional second-order CDW spots arranged around the second Bragg order. When doing this, care must be taken to consider potential distortions in the BFP that grow stronger with increasing distance from the optical axis of the microscope.

The second component is the specimen structure already discussed in section 6.1.5, allowing for stroboscopic driving of the NC/IC phase transition at repetition rates approaching the $\mathrm{MHz}$ regime. Compared to the $\leq 10 \mathrm{kHz}$ repetition rates used in related experiments, we gain another factor of $\geq 40$ in intensity. Lastly, the "Direct Electron DE-16" direct detector in counting mode used for the acquisition of all ultrafast DF images shown in this thesis plays an important role. Not only does the direct detector yield a DQE that is about two times higher than that of scintillator-based CCD cameras, but it also has a considerably higher SNR (373).

Taking these three factors together, we achieved an optimization of the integration time per DF image by a factor of $\geq 6000$. On the technical side, the crystallographic alignment of the specimen with respect to the orientation of the DF aperture array is achieved by using an in-plane rotation specimen holder (section 5.2.1). This is not absolutely critical for the success of the experiment, but nevertheless contributes significantly to its practicability.

Upcoming nanoimaging experiments will continue to profit from the general technical development of the UTEM method. Recently, a CEFID energy filter ("CEOS Energy Filtering and Imaging Device") has been installed at the Göttingen UTEM. The filter is equipped with an event-driven hybrid pixel detector (the "Timepix3"-based "ASI EM-CheeTah"). In the future, this unique combination will allow for the suppression of a large fraction of the inelastically scattered background intensity that is visible in the DF images while maintaining single-electron sensitivity at essentially background-free detection.

\subsubsection{DF imaging and electron beam coherence}

In parallel to the optimization of the integration times, the coherence of the source also plays a key role in the nanoimaging experiment. The individual $1.8 \mu \mathrm{m}$ apertures in the DF aperture array are significantly smaller than those conventionally used for DF imaging in TEMs. The reciprocal size of each of the apertures amounts to $\Delta q=0.27 / \mathrm{nm}$ (Fig. 6.5C). From this passband, we obtain the upper bound for the resolution of $1 / \Delta q \approx 5 \mathrm{~nm}$ given in section 5.2.2. And indeed, Fig. 5.11A confirms this value, because the NC/IC domain wall width extracted from both, the laser-heating and ultrafast nanoimaging experiments, exhibits a lower limit of $\sim 5 \mathrm{~nm}$. An upper limit to the actual microscopic width of a domain wall should be given by the $\sim 2 \mathrm{~nm}$ width of 

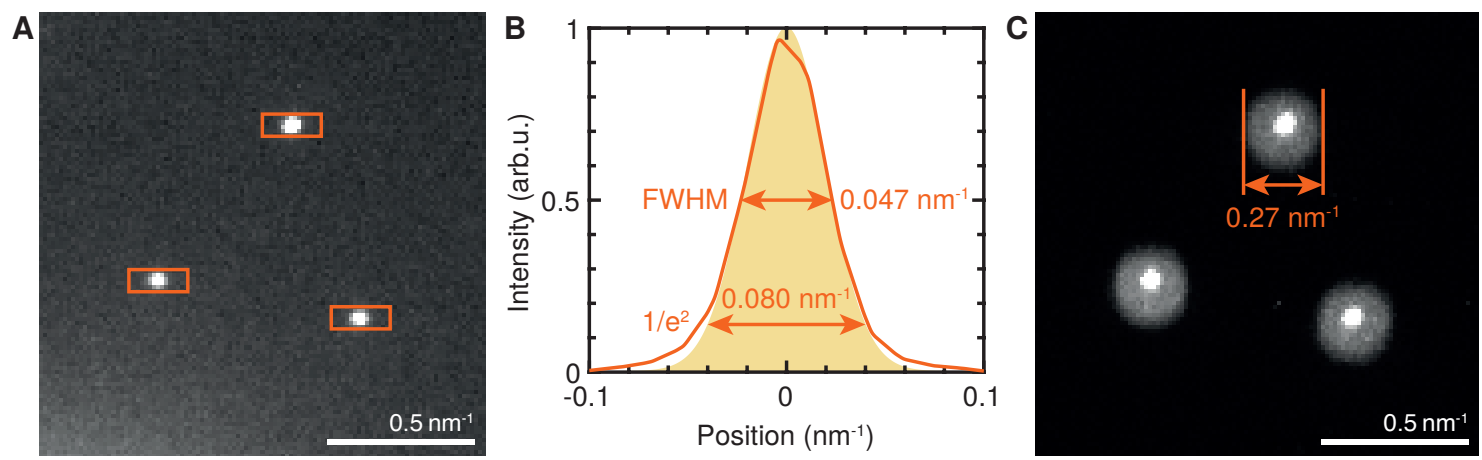

Figure 6.5: Determination of CDW spot width and DF aperture diameter. (A) Close-up of a second-order CDW spot triplet in the diffraction pattern shown in the top panel of Fig. 5.1C. The orange boxes indicate line-out positions for the CDW spot width determination in B. (B) CDW spot profile averaged over nine selected second-order spots (orange line) and Gaussian fit for spot width determination (yellow area). The resulting FWHM and $1 / e^{2}$ spot widths are indicated. (C) Close-up of the same spot triplet as in A in the apertured diffraction pattern shown in the bottom panel of Fig. 5.1C. The individual aperture diameter, averaged over the same nine spots as in $\mathrm{B}$, is indicated.

the discommensurations in the equilibrium NC phase (9). This supports the interpretation that the resolution of the nanoimaging experiment is limited by the DF aperture diameter.

At the same time, the CDW spots have a FWHM width of $\Delta q=0.047 / \mathrm{nm}$ in static diffraction images taken with photoelectrons, resulting in a lower limit to the electron beam coherence length of $1 / \Delta q \approx 22 \mathrm{~nm}$ in DF imaging mode (Fig. 6.5A and B). Specifically, this value is not influenced by the NC CDW in-plane correlation length because we measure an almost identical FWHM of $0.044 / \mathrm{nm}$ for the Bragg spots. Based on the lattice constant of $1 T-\mathrm{TaS}_{2}$, the coherence length is sufficient to illuminate an area of 3900 undistorted unit cells, leading to an adequate sampling despite the incommensurability of the NC CDW.

In the terms of the publication describing the gun of the Göttingen UTEM (66), the DF imaging mode uses spot 1 , a custom alpha setting close to alpha 1 and the largest condenser aperture CLA 1 (close to TEM-111; high coherence setting at $120 \mathrm{kV}$ acceleration voltage). Due to the small-diameter collimated beam in this setting, there are no significant objective lens aberrations which would broaden the Bragg and CDW spots. Instead, the lower limit to the coherence length should be very close to its actual value. From the coherence length and the illumination diameter of $2.4 \mu \mathrm{m}$ (section 5.2.1), we obtain an estimate of $0.91 \%$ for the degree of coherence. We can compare this result to a published reference value: In TEM-111 at $200 \mathrm{kV}$ acceleration voltage (high coherence setting), the degree of coherence of the Göttingen UTEM was measured to be $1.43 \%$ (66). Since the degree of coherence generally is slightly lower at $120 \mathrm{kV}$ than at $200 \mathrm{kV}$, our measured value falls well in line.

In the design of a DF aperture array, it is of fundamental importance to choose the individual 
aperture diameter such that most of the superstructure spot intensity passes through the aperture, but the amount of parasitic background intensity transmitted around the spot is kept as small as possible. We chose to only reject intensities beyond three times the $1 / e^{2}$ diameter of the CDW peaks (Fig. 6.5B). This allows for a spatial misalignment of the DF aperture array on the order of the $1 / e^{2}$ spot width without losing useful CDW intensity at once (see Fig. 6.5C for an example of a slightly imperfect alignment).

From the degree of coherence in DF imaging mode, we derive a normalized emittance of $21 \mathrm{~nm} \cdot \mathrm{mrad}$, which is a useful value for comparison with other UTEM implementations. The emittance characterizes the average spread of position and momentum of an electron beam in phase space. The smaller the emittance, the higher the coherence of the electron beam $(66,374)$. It can be safely assumed that the nanoimaging experiment would be at the very limit of feasibility if the width of the CDW spots approached the diameter of the individual apertures, yielding a maximum normalized emittance of $\sim 120 \mathrm{~nm} \cdot \operatorname{mrad}$ allowing for use of the DF aperture array as presented in Fig. 5.1B.

Thermionic UTEMs typically reach emittance values of $10^{4}$ to $10^{2} \mathrm{~nm} \cdot \operatorname{mrad}(375) \mathrm{such}$ that the above experimental requirement is hard to fulfill in this case. A typical workaround in a conventional TEM would be to spread the beam diameter in order to linearly increase the coherence length to the necessary amount. However, this results in a quadratic loss of beam current which is typically undesirable in UTEMs, ultimately illustrating the importance of the high-coherence source of the Göttingen UTEM in the demonstrated DF imaging approach.

\subsubsection{The role of probabilistic domain switching in the NC phase}

As outlined in section 3.4, both the $\mathrm{NC}$ and $\mathrm{C}$ phase of $1 T-\mathrm{TaS}_{2}$ can be aligned with the lattice in two symmetry-equivalent ways. In case of the NC phase, this leads to the formation of $\alpha$ and $\beta$ mirror domains aligned with the underlying hexagonal lattice at an angle of approximately $\pm 12^{\circ}$. Typically, a single $\alpha$ or $\beta$ domain extends over the whole crystal or thin film (12). This behavior is also observed in the thin films obtained by ultramicrotomy and has been attributed to lattice strain, effectively lifting the degeneracy between $\alpha$ and $\beta$ states (265).

In an early experiment, we noticed that intense single-shot laser pulses can be used to induce switching between $\alpha$ and $\beta$ orientations. Figure 6.6 shows room-temperature DF micrographs that have been acquired using a conventional aperture selecting $\mathrm{NC} / \alpha$ phase superstructure reflections. Initially, the specimen exhibits $\alpha$ and $\beta$ domains (Fig. 6.6A). Over the course of the experiment, additional images of the same specimen region have been taken after interaction 


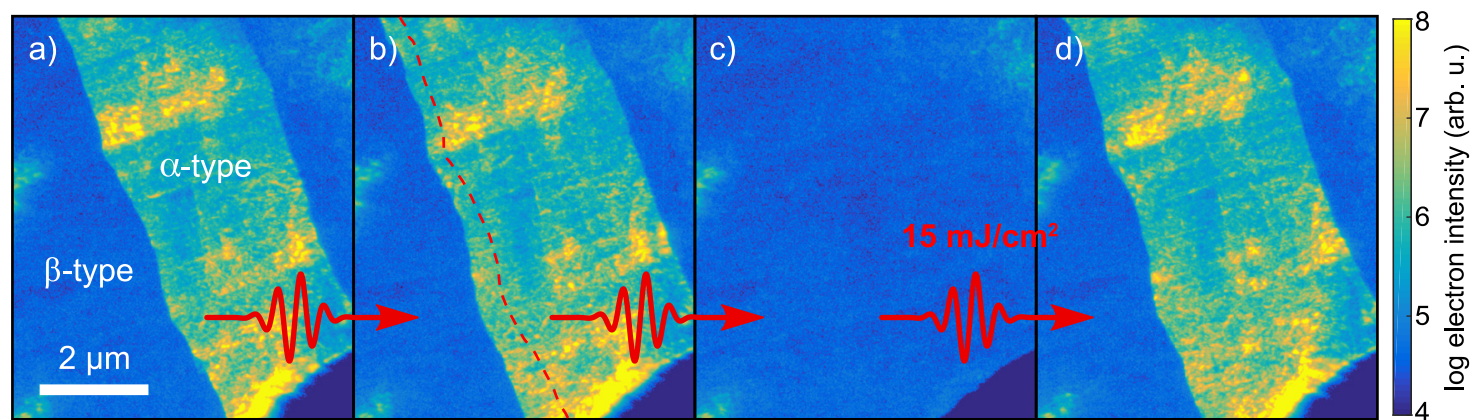

Figure 6.6: Single-pulse domain switching in the NC phase of $1 T-\mathrm{TaS}_{2}$. Conventional DF images of an ultramicrotomed $1 T-\mathrm{TaS}_{2}$ film in false-color representation. An individual circular DF aperture has been placed such that $\alpha$-type mirror domains show high image intensity while there is virtually no intensity in $\beta$-type regions. Between the individual panels (A) to (D), single ultrashort laser pulses $\left(15 \mathrm{~mJ} / \mathrm{cm}^{2}\right.$ at $\left.800 \mathrm{~nm}\right)$ have interacted with the specimen and, in turn, modified the mirror domain structure. The dark region in the bottom right corner of each image shows part of the supporting copper grid. Reprinted from Ref. (376) with permission from the author.

with individual, intense laser pulses. This induced either a slight change in domain shape (from panel A to B) or even led to the complete dis- and reappearance of individual domains (from panel B over $\mathrm{C}$ to $\mathrm{D}$ ). The emergence of the $\alpha$ domain may be seeded by the copper specimen grid visible in the bottom right corner (376).

A more detailed investigation of this probabilistic switching behavior has been conducted by ZoNG et al. using TEM and UED. They have found that the probability of creating a $\beta$ domain within a specimen in the $\alpha$ state (the injection of a domain wall) increases with laser fluence. In contrast, the probability of restoring the initial $\alpha$ state decreases with increasing fluence. The current understanding of this behavior is that a higher excitation fluence increases the density of topological defects in the nascent IC phase due to a large number of independent nucleation sites (see also section 3.5). When cooling down to the NC phase again, the different phases of the charge-density modulation at IC nucleation sites lead to a higher probability of the appearance of the second symmetry-equivalent NC phase. With a smaller number of topological defects induced, lower fluences lead to a higher probability of restoring the initial configuration (265).

This behavior can potentially lead to difficulties in ultrafast experiments. With two degenerate $\alpha$ and $\beta$ ground states, the reversibility requirement for the stroboscopic approach is, strictly speaking, not fulfilled. When driving this kind of bistable phase transition between the $\mathrm{NC}$ and IC phases of $1 T-\mathrm{TaS}_{2}$, the two possible configurations of the NC phase are visible simultaneously in the diffraction pattern as reported in Ref. (377). While both ground states can be separately analyzed in UED experiments, any instance in which the specimen relaxes into the "other" configuration leads to a loss of DF image intensity in the nanoimaging experiment. 
Conveniently, we did not observe this kind of switching behavior in our preceding ultrafast diffraction experiments using the same specimen structure (378). This can be attributed to two main reasons: Firstly, the pump fluences in the nanoimaging experiment ranged between $2.6 \mathrm{~mJ} / \mathrm{cm}^{2}$ (Fig. 5.2A) and $6.3 \mathrm{~mJ} / \mathrm{cm}^{2}$ (Fig. 5.7), while the switching threshold at room temperature was found to be $\sim 5 \mathrm{~mJ} / \mathrm{cm}^{2}$ (265). Therefore, the switching probability is low even in our highest fluence case. And secondly, the formation of $\beta$ domains in an $\alpha$ ground state takes places on length scales that are similar or larger than the circular $1.85 \mu \mathrm{m}$ region of interest in the nanoimaging experiment (cf. Ref. (265) and Fig. 6.6). Simultaneously, the specimen outside of the circular gold aperture remains in the $\mathrm{NC}$ phase at all delay times due to the confinement of the optical excitation (see the bright circular rim in Fig. 5.2A at $\Delta t>0$ ). This leads to a high probability that, upon cooling, the original $\mathrm{NC}$ phase orientation grows back into the IC regions. Thus, the influence of independent nucleation sites is fully suppressed in the nanoimaging experiment.

\subsubsection{A note on CDW phases and domain walls}

The mirror domain walls between $\alpha$ and $\beta$ configurations are just one example of a larger number of different phenomena that are subsumed under the term domain wall. In the descriptions of the nanoimaging experiment in Chapter 5, we use the term to describe the phase boundaries between $\mathrm{NC}$ and IC regions in $1 T-\mathrm{TaS}_{2}$. These domain walls are a non-equilibrium phenomenon due to the underlying spatially inhomogeneous temperature distribution. At every point of the specimen, the local temperature only allows for one stable phase, NC or IC (at least outside of the very narrow hysteresis; see section 5.2.11). In contrast, mirror domain walls are line defects in the CDW superlattice of the crystal. The $\alpha$ and $\beta$ configurations on both sides of the mirror domain wall represent thermodynamically stable CDW phases.

The bistability is also reflected by the free-energy landscape we used for the time-dependent Ginzburg-Landau simulations (Fig. 5.4B). At room temperature, the potential has a double-well shape with equal minima at an order parameter of $\phi= \pm 1$, corresponding to the $\alpha$ and $\beta$ configurations. Only upon the transition into the IC phase, both minima merge to form the IC phase minimum at $\phi=0$ while the symmetry of the material is being restored. Due to the first time derivative of the order parameter in the equation of motion (Eq. 5.1), the order parameter only asymptotically approaches the minimum at $\phi=0$. Therefore, a relaxation of the simulation into the $\beta$ minimum at $\phi=-1$ is precluded. Would a fluctuation term be included in the model, it could be expanded to cover situations involving $\alpha / \beta$ switching behavior. However, 
for describing coherent behavior such as oscillations of the amplitude mode, one would also need to add a second time derivative to the equation of motion as outlined in section 3.3.

Generally, domain walls in themselves already contain some intricate physics. While it has been hypothesized that mirror domain walls in the $\mathrm{C}$ phase of $1 T-\mathrm{TaS}_{2}$ may host conducting channels (265), domain walls can also be induced within a single configuration of the $\mathrm{C}$ phase using current pulses from an STM tip. At these domain walls, the phase of the charge-density modulation changes abruptly, leading to very different properties depending on the specific structure and orientation of the domain wall. Such domain walls can exhibit in-gap states that in fact may lead to metallic behavior $(15,16,379,380)$.

\subsubsection{Ultrafast nanoimaging of electronic order parameters}

In Chapter 5, we demonstrated a combined $530 \mathrm{fs}$ temporal and $5 \mathrm{~nm}$ spatial imaging resolution with a contrast tailored to map the structural order parameter of the NC/IC phase transition in $1 T-\mathrm{TaS}_{2}$. While the DF imaging approach is unique in its structural imaging capabilities, there are a number of related techniques that already are or may in the near future be able to spatially probe the ultrafast electronic component of correlated phase transitions.

A recent demonstration of those capabilities uses inelastic electron-light scattering to conduct photon-induced near-field electron microscopy (PINEM) of an individual $\mathrm{VO}_{2}$ nanowire in an UTEM (Fig. 6.7A and B, top panel) (168). An ultrashort gating laser pulse induces an optical near-field around the nanowire (Fig. 6.7B, center panel). As a consequence, the electron beam undergoes inelastic scattering into a number of well-defined energy sidebands towards the gain and loss sides of the spectrum. Specifically, the near-field intensity encodes the dielectric function of the material, leading to a PINEM contrast between the insulating room-temperature and metallic high-temperature phases below and above $340 \mathrm{~K}$, respectively.

When a second pump laser pulse is introduced, the temporal response of the dielectric function can be probed in a stroboscopic experiment (Fig. 6.7C and D). The gating pulse is in overlap with the electron probe pulse at all times and enables the near-field interaction. The polarization of the pump pulse is chosen such as to minimize interaction with the electron pulse (Fig. 6.7B, bottom panel). Advantageously, the temporal resolution is determined by the $50 \mathrm{fs}$ duration of the optical gating and pump pulses - not by the duration of the electron pulse as it is usually the case. However, the effective electron current yielding contrast is reduced by the ratio of gating and electron pulse duration. The spatial resolution of the energy-filtered PINEM maps is on the order of $20 \mathrm{~nm}(168)$. 
A

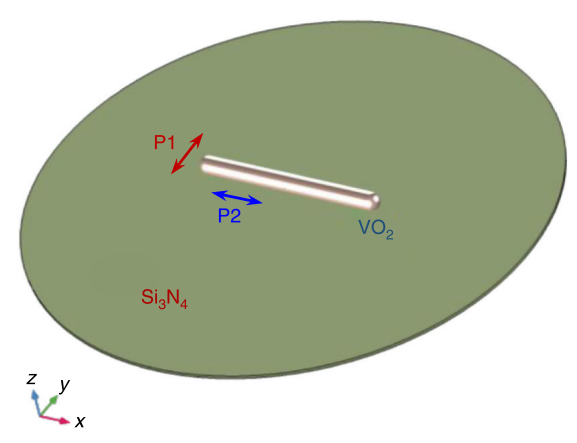

B

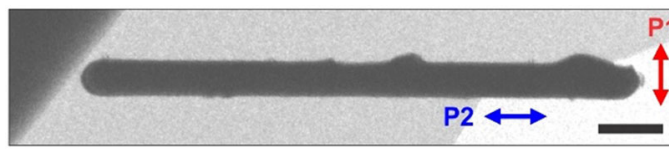

Bright-field image

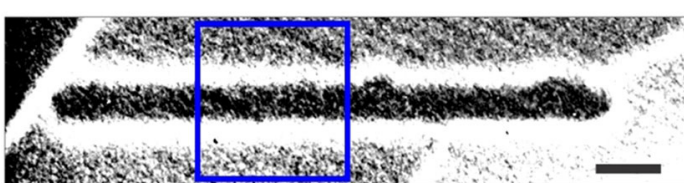

PINEM map at $\lambda=800 \mathrm{~nm}$

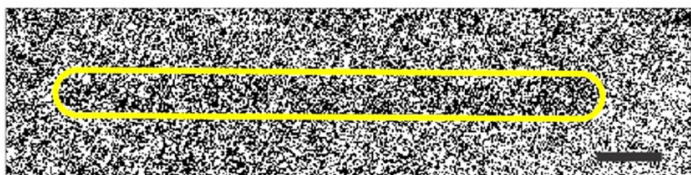

PINEM map at $\lambda=400 \mathrm{~nm}$
C

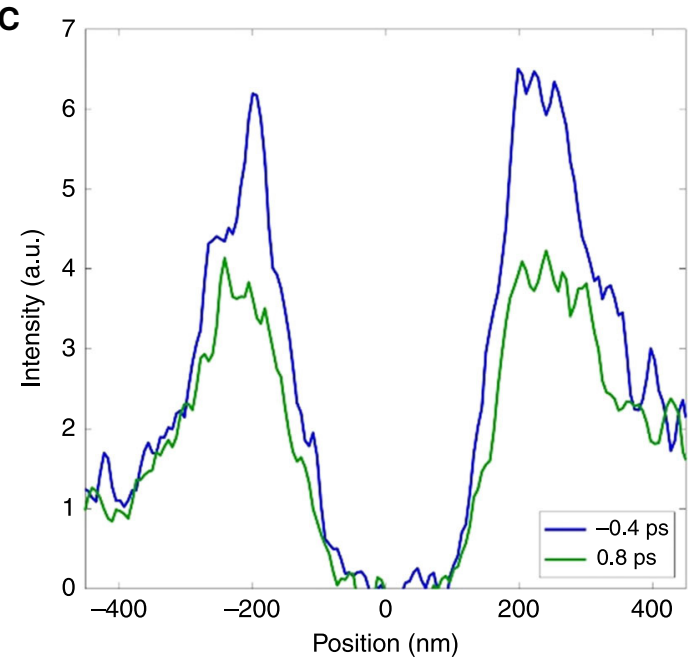

D

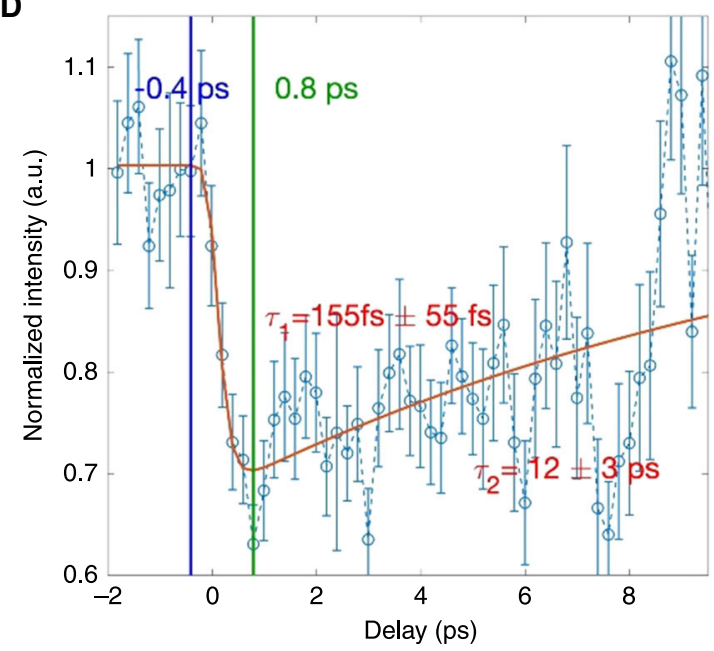

Figure 6.7: Two-color PINEM probing of electronic dynamics in $\mathrm{VO}_{2}$. (A) Rendering of the $\mathrm{VO}_{2}$ nanowire geometry on a silicon nitride membrane. The polarization of the $800 \mathrm{~nm}$ gating pulse P1 (red) and of the $400 \mathrm{~nm}$ pump pulse P2 (blue) is indicated. (B) Top: BF micrograph of the nanowire with the polarization of both pulses indicated. Center: PINEM map of the nanowire formed by energy-filtering electrons that have been gain-scattered due to interaction with the gating pulse P1. Bottom: PINEM map due to interaction solely with the pump pulse P2. No inelastically scattered intensity is visible by choice of the pulse polarization. (C) PINEM line scan across the nanowire as indicated by the blue box in B for two temporal delays $\Delta t$ between optical pump and electron probe pulse. The gating pulse is in overlap with the probe pulse at all times. The response of the specimen to the optical excitation is reflected by the different PINEM intensities before and after time-zero. (D) Spatially integrated PINEM signal as a function of temporal delay. The observed decay and recovery can be described using a biexponential model. Reprinted from Ref. (168). Licensed under CC BY 4.0.

In scanning probe approaches, the optical near-field scattered by an oscillating nanometric tip is used to form images of the free-carrier response of the specimen (scanning near-field optical microscopy or SNOM). Ultrafast variants of this technique have been successfully applied to map the MIT of small $\mathrm{VO}_{2}$ crystals and nanowires $(43,44)$. In the experiment in Ref. (44), ultrashort pump and probe laser pulses are focused onto the scanning tip with a polarization parallel to the shaft. Figure $6.8 \mathrm{~B}$ schematically shows the scanning of the tip from the substrate 

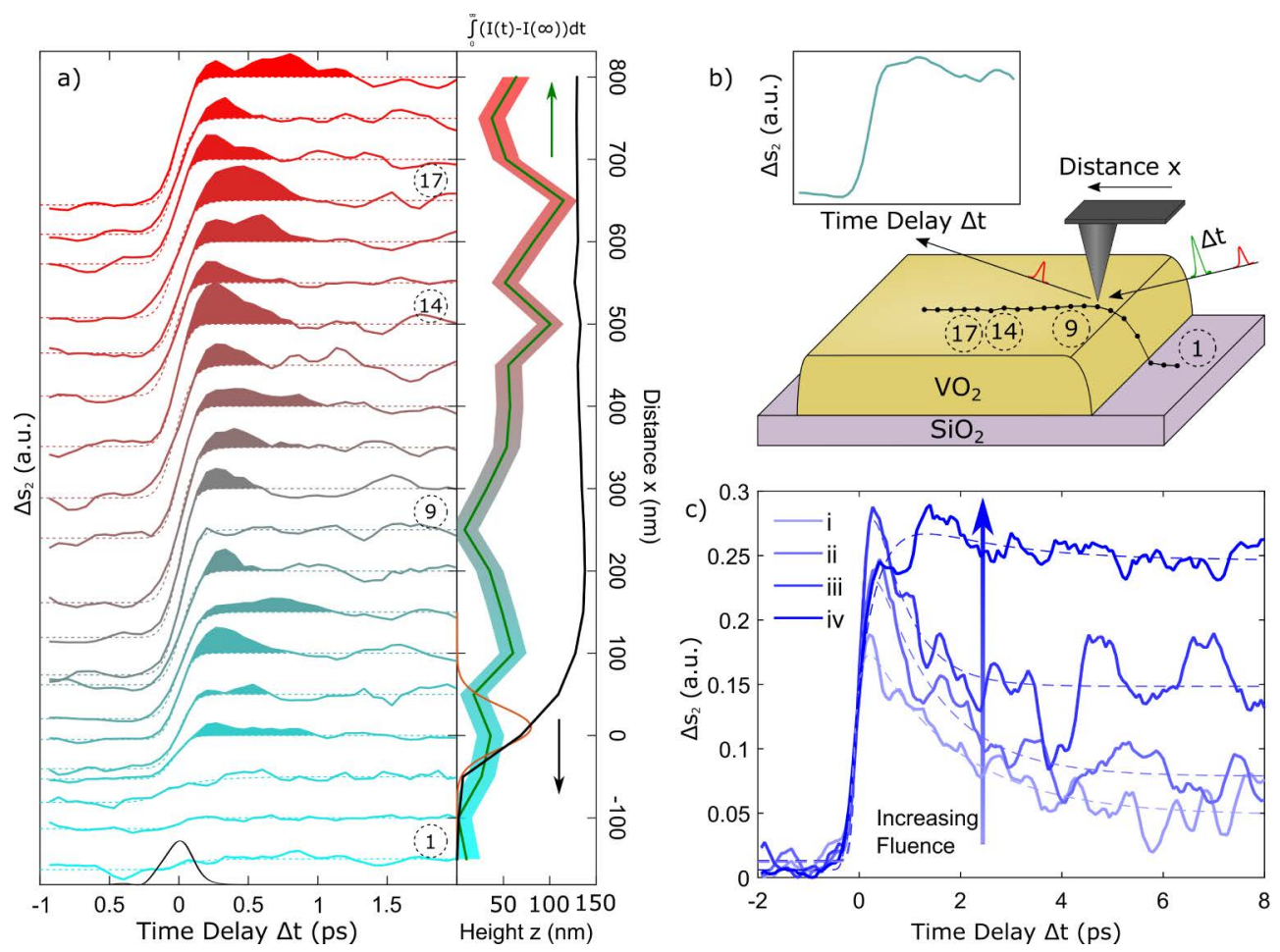

Figure 6.8: Scanning near-field optical microscopy of electronic dynamics in $\mathrm{VO}_{2}$. (A) Left: Change in tip-scattered intensity $\Delta s_{2}$ as a function of the position on the specimen (see B) and the temporal delay $\Delta t$ between optical pump and probe pulses. The specific response varies strongly across the crystal. The filled areas in some of the traces indicate an overshoot and fast relaxation after time-zero. Right: Height profile of the crystal (black) and integral of the filled areas (colors corresponding to the left panel). The temporal and spatial resolution is indicated by a Gaussian in both panels. (B) Schematic of the tip scanning across the crystal, starting from the substrate. At each point, a delay curve (see A) is acquired. (C) Four delay curves as a function of pump fluence illustrate different regimes of photoinduced dynamics. Reprinted with permission from Ref. (44). Copyright 2016 American Chemical Society.

onto a $\mathrm{VO}_{2}$ crystal, acquiring a temporal delay curve at every point (Fig. 6.8A). Depending on the pump fluence and the position on the crystal, behavior ranging from a quickly decaying electronic excitation to a photoinduced transition into the metallic phase is observed (Fig. 6.8C). The spatial resolution of this technique is limited by the 10 to $20 \mathrm{~nm}$ radius of the tip apex, while the few-100 fs temporal resolution is determined by the optical pulse duration.

There is one major difference between this kind of SNOM-based nanoimaging and the DFbased nanoimaging technique introduced in the present thesis: While the DF approach uses far-field excitation of the full specimen structure, the near-field excitation in the time-resolved SNOM experiment in Fig. 6.8 is scanned with the tip. Thus, the obtained specimen response is local and allows for insights into the specimen properties as modified, e.g., by local strain between $\mathrm{VO}_{2}$ and the substrate (44). However, ultrafast SNOM can also be combined with 
far-field excitation of the region of interest. In Ref. (43), this is realized by polarizing the pump pulses perpendicularly to the tip shaft.

To the author's knowledge, there is no published experiment demonstrating ultrafast nanoimaging of the electronic component of a phase transition in $1 T-\mathrm{TaS}_{2}$. Still, in a static SNOM experiment, the transition between the $\mathrm{C}$ and $\mathrm{NC}$ phase has been investigated (46). Interestingly, a $450 \mathrm{~nm}$ broad spatial transition region between $\mathrm{C}$ and $\mathrm{NC}$ phase regions was observed in the experiment. Supported by Ginzburg-Landau theory, this behavior has been interpreted in terms of a non-equilibrium CDW order across the $\mathrm{NC} / \mathrm{C}$ phase boundary which gives rise to the observed gradual transition in metallicity. On a microscopic level, the transition is suggested to involve a change in size of the $\mathrm{C}$-type domains making up the $\mathrm{NC}$ phase. While a large number of other non-equilibrium states is known in $1 T-\mathrm{TaS}_{2}$ (section 3.5), this would be the only one accessible without using ultrashort stimuli.

Finally, time-resolved photoemission electron microscopy (PEEM) $(51,54)$ has a great potential for representing an electronic counterpart to our structure-sensitive technique. In a number of experiments, PEEM has demonstrated its capabilities of imaging the dynamics of charge carriers after optical excitation $(32,53,55)$. These instruments achieve down to $50 \mathrm{~nm}$ spatial and $30 \mathrm{fs}$ temporal resolution (55). With the correction of chromatic and spherical aberrations, few-nm resolution can be obtained in static PEEMs $(381,382)$.

Most intriguingly, state-of-the-art time-resolved PEEMs are also momentum microscopes that can switch between real-space and reciprocal-space imaging $(383,384)$. And the analogy to TEMs does not end there: By inserting an aperture into the image plane, band structure information can be extracted from a selected area of the specimen. Conversely, an aperture in the BFP can be used to control the contrast in real-space images. Additionally, energy filtering can be used to only take photoelectrons into account that were emitted from certain binding energies. Both filtering techniques combined would perspectively allow for tailoring the contrast of ultrafast DF PEEM images to precisely map the temporal evolution of CDW gaps in the band structure of $1 T-\mathrm{TaS}_{2}$ (cf. Fig. 3.7).

\subsubsection{Ultrafast DF imaging and 4D-STEM}

An alternative approach capable of structural nanoimaging is scanning transmission electron microscopy (STEM). Conventional STEM images are acquired by scanning a focused electron beam across the specimen, and recording a BF or DF signal as a function of position using a semiconductor detector $(73,385)$. The obtained atomic-scale information can be further 
expanded by acquiring a full diffraction pattern at every point of the specimen using a pixelated detector instead of the integrated signal. This enables access to structural information such as composition, orientation and strain, as well as local electromagnetic fields (76, 386, 387). Because of the four-dimensional nature of the recorded datasets (two real-space and two reciprocal dimensions), this technique is commonly called 4D-STEM (387).

In combination with UTEM, time-resolved 4D-STEM promises femtosecond time resolution with powerful contrast tailoring options in post-processing. A first impression of these capabilities has been given by FEIST et al. In this work, the strain field in a graphite film was reconstructed as a function of temporal delay from Bragg line shifts in convergent-beam electron diffraction patterns (158). As reported by SuNG et al. in a recent preprint, static 4D-STEM can also be applied to map the CDW order parameter in $1 T-\mathrm{TaS}_{2}$ with $5 \mathrm{~nm}$ spatial resolution. To this end, virtual DF images are formed by applying a mask similar to our DF aperture array to the individual diffraction patterns and summing up the masked intensities (31). However, no nanoimaging using time-resolved 4D-STEM has been demonstrated to date.

That is because achieving the combination of high temporal, spatial and reciprocal resolution as well as sufficient SNR is challenging. The use of a convergent electron beam spreads the spot intensities in reciprocal space. This decreases the SNR of the CDW superstructure reflections compared to parallel-beam diffractograms due to the mixing of spot and background intensities within the virtual mask. Additionally, diffraction discs start to overlap already at moderate convergence angles which leads to interference effects and complicates the generation of virtual DF images (388). At $80 \mathrm{kV}$ acceleration voltage, second-order NC spots start to overlap at a convergence semi-angle of $1.7 \mathrm{mrad}$ (cf. Fig. 6.5A), while the overlap between second-order $\mathrm{NC}$ and IC spots already sets in at $0.7 \mathrm{mrad}$ (cf. Fig. 5.5). This leads to a loss of contrast to the NC/IC phase transition, and thus explains the relatively small convergence semi-angle of $0.55 \mathrm{mrad}$ at $80 \mathrm{kV}$ used in Ref. (31). Typical convergence semi-angles for STEM in uncorrected microscopes are on the order of $10 \mathrm{mrad}(88)$.

Convergence angles can be linearly reduced by using smaller condenser aperture diameters, leading to a quadratic loss of beam current which is undesirable in a stroboscopic experiment, or by slightly spreading the electron beam, effectively reducing spatial resolution. Newer TEM models allow for an increased degree of control over the beam convergence by implementing a four-lens condenser system. This certainly opens up perspectives for ultrafast nanoimaging using 4D-STEM techniques. A related time-resolved x-ray diffraction microscopy technique has already been shown to resolve the mesoscopic phase progression in photoexcited $\mathrm{VO}_{2}$, but with only $350 \mathrm{~nm}$ spatial and 100 ps temporal resolution (389). 


\subsubsection{Perspectives for future nanoimaging experiments}

In the future, the ultrafast nanoimaging approach presented in this thesis can be incorporated into novel experimental schemes and applied to different materials. For this purpose, the DF aperture array can be easily adapted to obtain sensitivity, e.g., to the structural order parameter of the MIT in $\mathrm{VO}_{2}$. Most intriguingly, the PINEM approach presented in Ref. (168) could be combined with ultrafast DF imaging to yield quasi-simultaneous information about lattice and carrier dynamics, playing out the full strength of the UTEM approach. This is particularly appealing because the long-standing question about the specific role of Mott and Peierls contributions to the MIT has still not been conclusively clarified $(24,45,64,131,169-175,390-392)$.

$\mathrm{A} \mathrm{VO}_{2}$ specimen for high-repetition-rate experiments can be prepared using the sol-gel method as demonstrated in early UTEM experiments in Refs. $(131,169)$. When prepared directly on silicon nitride membranes, the resulting polycrystalline films are ready for TEM inspection and show the expected change in crystal symmetry across the phase transition (Fig. 6.9A and B). Besides, lift-off and transfer procedures are possible as well and allow for free placement of the $\mathrm{VO}_{2}$ film $(131,169)$, for example on top of a prepared gold aperture.

Alternatively, electron-lithographic processes would allow for structuring isolated, polycrystalline $\mathrm{VO}_{2}$ islands on a silicon nitride membrane. In the past, a similar approach has proven
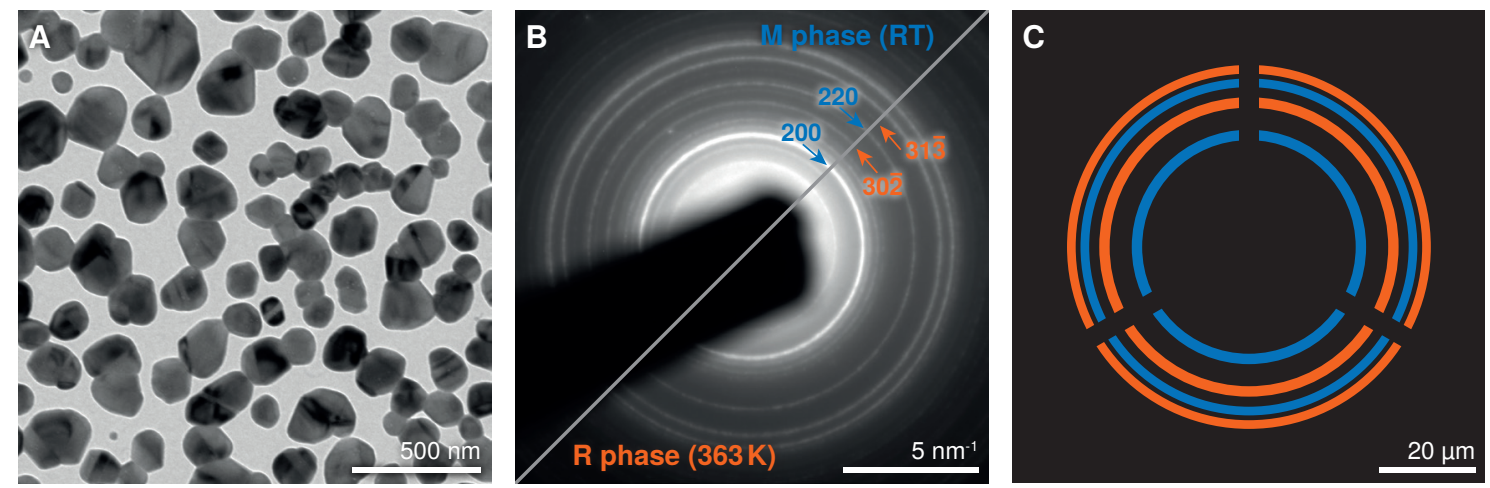

Figure 6.9: Proposal of DF apertures for polycrystalline $\mathrm{VO}_{2}$. (A) $\mathrm{BF}$ micrograph of $\mathrm{VO}_{2}$ single crystals on a silicon nitride membrane at room temperature. The crystals were prepared using the sol-gel method (393). (B) Diffraction patterns taken in the monoclinic (M) room-temperature phase (top) and in the rutile (R) high-temperature phase at $363 \mathrm{~K}$ (bottom). Due to the higher symmetry in the high-temperature phase, a number of distinct diffraction orders vanishes across the phase transition. The most isolated vanishing diffraction

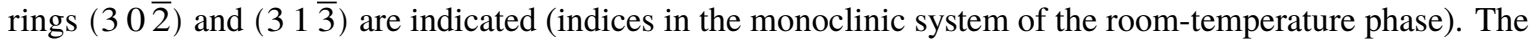
intensity of these rings can be considered as an order parameter of the transition from the $\mathrm{M}$ to the $\mathrm{R}$ phase. An increase in intensity in the $(200)$ and $(220)$ rings could indicate a transition into a photoinduced monoclinic metallic $(\mathscr{M})$ phase (see main text). (C) Proposal of two complementary DF apertures based on the $(30 \overline{2})$

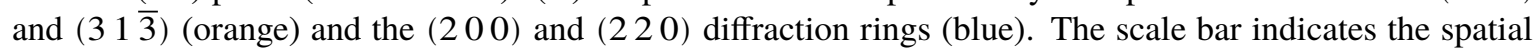
dimensions required for use in the Göttingen UTEM. 
to be successful in an ultrafast demagnetization experiment (102). As silicon nitride is almost transparent at $800 \mathrm{~nm}$ wavelength, this confines the optical excitation to the individual islands. Two different DF apertures as shown in Fig. 6.9C could then be used to spatially differentiate between two distinct phase transition regimes: the transition into the thermodynamically stable rutile high-temperature phase (orange aperture) and into a proposed photoinduced monoclinic metallic phase (blue aperture) $(24,170,394)$.

Apart from this specific experimental proposal, tailored DF apertures can in principle be applied to any material system that exhibits the dynamic formation or melting of superstructure features. This includes, for example, the formation of CDWs in other TMDCs such as $1 T-\mathrm{TiSe}_{2}$, which exhibits a C CDW transition temperature of $202 \mathrm{~K}$ (395). This transition is still accessible in UTEM experiments without overly large experimental overhead.

Recently, the $\mathrm{RTe}_{3}$ class of layered materials (rare-earth tritellurides) has attracted considerable attention in experiments using various ultrafast probes due to its symmetry-breaking phase transitions into unidirectional stripe-ordered CDW states $(26,29,223,227,229,268,396)$. In $\mathrm{LaTe}_{3}$, a specific member of that material class, CDW formation occurs up to temperatures of $\sim 670 \mathrm{~K}$, and a small lattice anisotropy can be exploited to optically control the direction of the charge-density modulation under non-equilibrium conditions (see Chapter 1 and Fig. 1.1C) (26). Other types of long-range order also generate superstructure diffraction signatures that are suitable for the DF imaging approach, such as the charge- and orbital-ordered phase in $\mathrm{Pr}_{0.7} \mathrm{Ca}_{0.3} \mathrm{MnO}_{3}$ below a transition temperature of $240 \mathrm{~K}$ (Fig. 2.6D) $(21,397)$.

Returning to $1 T-\mathrm{TaS}_{2}$, current-induced picosecond switching between different insulating and metallic states has been demonstrated in the past $(257,258,316,398)$. Based on the large changes in resistivity and the high switching speed, new concepts for $1 T-\mathrm{TaS}_{2}$-based non-volatile memory devices have been proposed $(257,258,316)$. Provided that picosecond current pulses can be triggered in situ using a photoconductive switch (399), the experimental scheme can be transformed into a stroboscopic UTEM experiment.

Additionally, ongoing progress in the development of UTEM sources (400) and pulse compression schemes using radiofrequency $(139,401-403)$ and terahertz fields (404-406) will in the future routinely allow for generating sub-100 fs electron pulses with an increased bunch charge. This will ultimately allow to resolve coherent features such as oscillations of the CDW amplitude mode, considerably reduce integration times, and bring the DF imaging technique a giant step closer towards the imaging of actual devices. 


\subsection{Concluding remarks}

The present thesis reports on the development and application of a tailored ultrafast electron microscopy scheme. Applied to the NC/IC CDW phase transition in $1 T-\mathrm{TaS}_{2}$, this scheme has delivered the first real-space observation of transient domain formation in a CDW system. Furthermore, this work represents

- the first demonstration of ultrafast nanoimaging of a structural phase transition with femtosecond temporal and $5 \mathrm{~nm}$ spatial resolution,

- the realization of stroboscopic probing of a structural phase transition at hundreds of kilohertz repetition rates,

- the first implementation of tailored contrast enhancement in ultrafast electron microscopy,

- as well as the most direct illustration of the drastic differences in the domain pattern between continuous and pulsed laser excitation.

This set of achievements has only become possible by integrating a number of unique components. By the use of the DF aperture array, we obtained sensitivity to the structural order parameter of the NC/IC phase transition in $1 T-\mathrm{TaS}_{2}$. Together with an optimized specimen design and the high DQE of a direct detector, we reduced the integration time by almost four orders of magnitude with respect to more unfavorable conditions. Additionally, the high-coherence source of the Göttingen UTEM allowed for combining spatiotemporal resolution with a normalized emittance of $21 \mathrm{~nm} \cdot \mathrm{mrad}$. This permitted the use of an individual aperture diameter of $1.8 \mu \mathrm{m}$ in the DF aperture array-considerably smaller than conventional objective lens apertures.

Furthermore, we presented time-dependent Ginzburg-Landau simulations that reproduce the domain formation and domain wall dynamics observed in the ultrafast experiment. The parameters for the time-dependent simulations have been extracted from the domain patterns of the steady-state heating experiment in a self-contained approach. In this manner, we have shown that ultrafast DF imaging allows for intriguing insights into the interplay of order parameter dynamics and nanoscale thermal transport.

Previously, the nanoscale imaging of order parameter dynamics was mostly limited to electronic and magnetic degrees of freedom. With the present work, the spatiotemporal imaging of structural processes on femtosecond time and nanometer length scales has finally moved into reach. Allowing for sensitivity to further structural degrees of freedom in complex materials, our approach will contribute to a better understanding of actively controlled processes in energy conversion devices. 


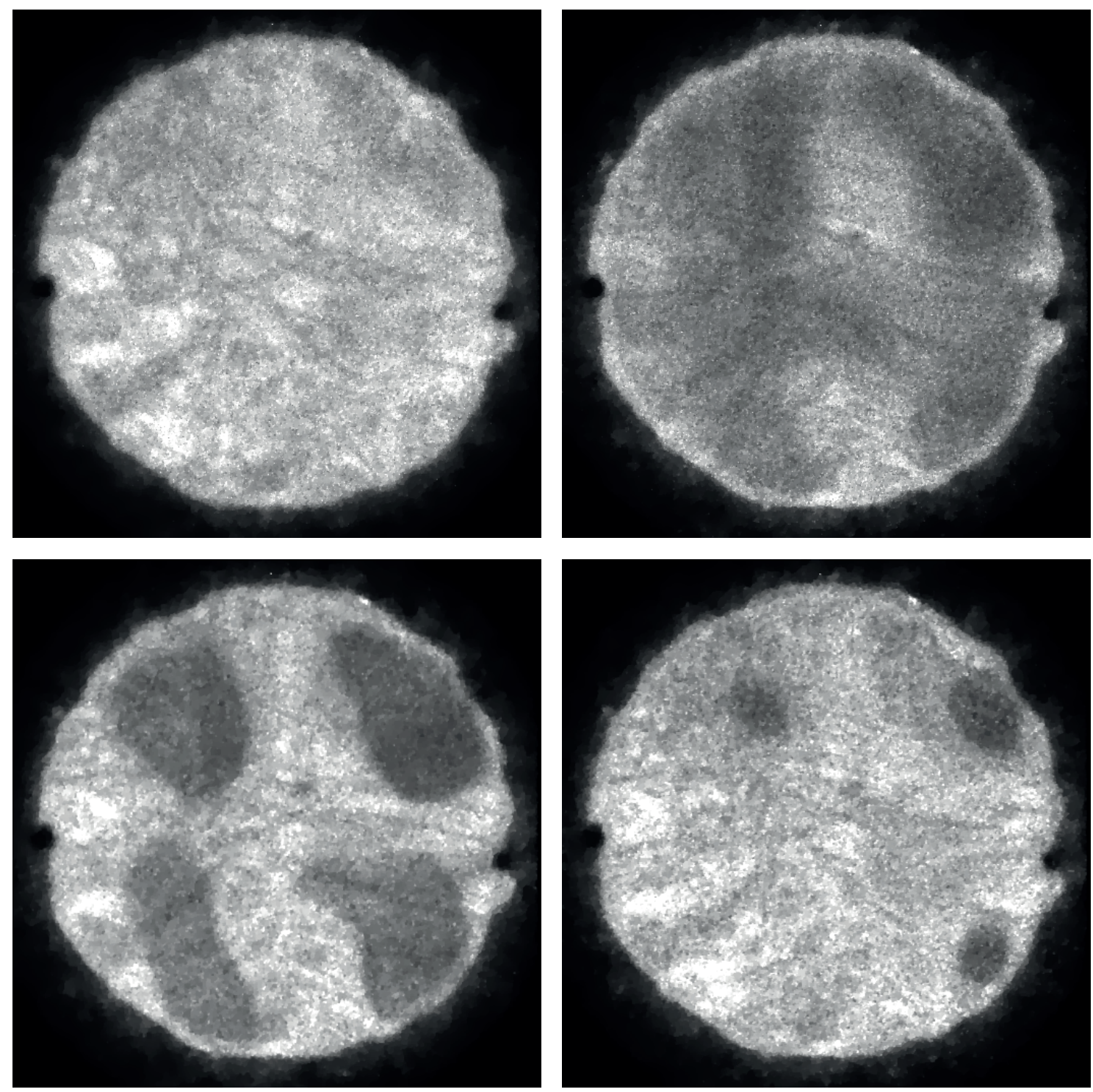

Ultrafast dark-field images of charge-density wave dynamics in tantalum disulfide. Top left to bottom right: Temporal delays of -5 ps, 1 ps, 1500 ps and 3141 ps. See Figure 5.2 for more details. 



\section{Appendix A}

\section{High-resolution prints of diffractograms}

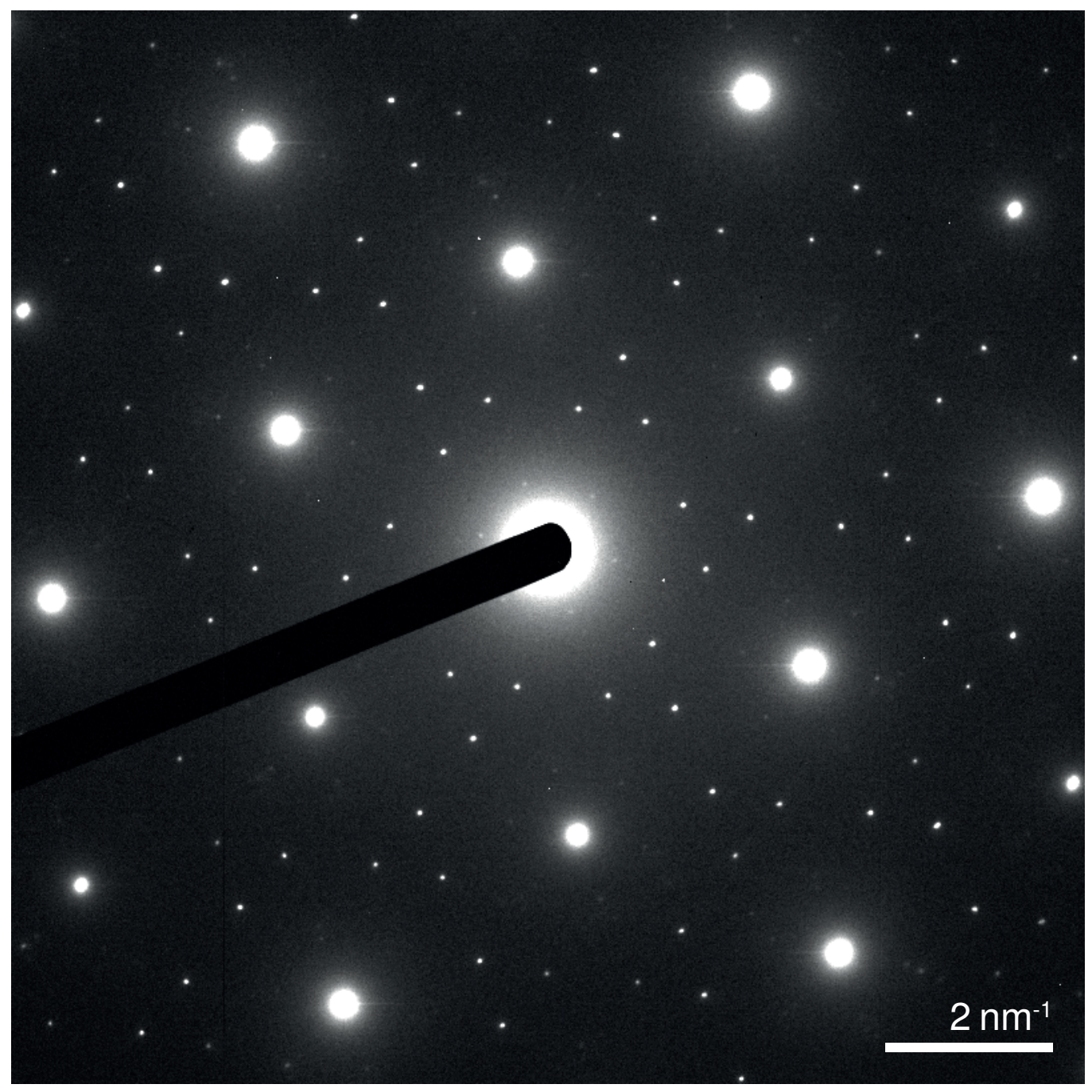

Diffractogram of the NC phase in $1 T-\mathrm{TaS}_{2}$ as shown in the top panel of Fig. 5.1C. 


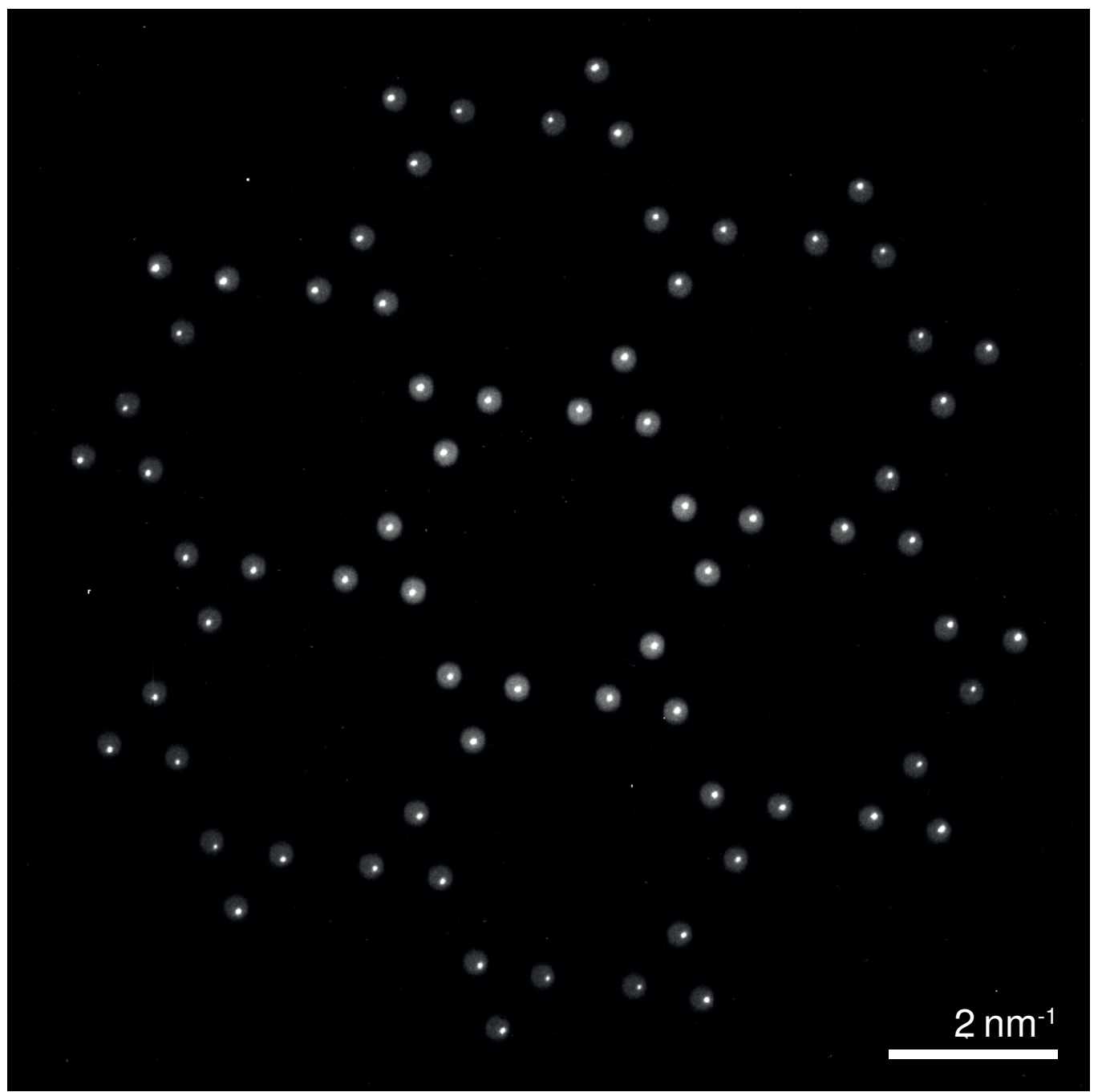

Apertured diffractogram of the NC phase in $1 T-\mathrm{TaS}_{2}$ as shown in the bottom panel of Fig. 5.1C. 


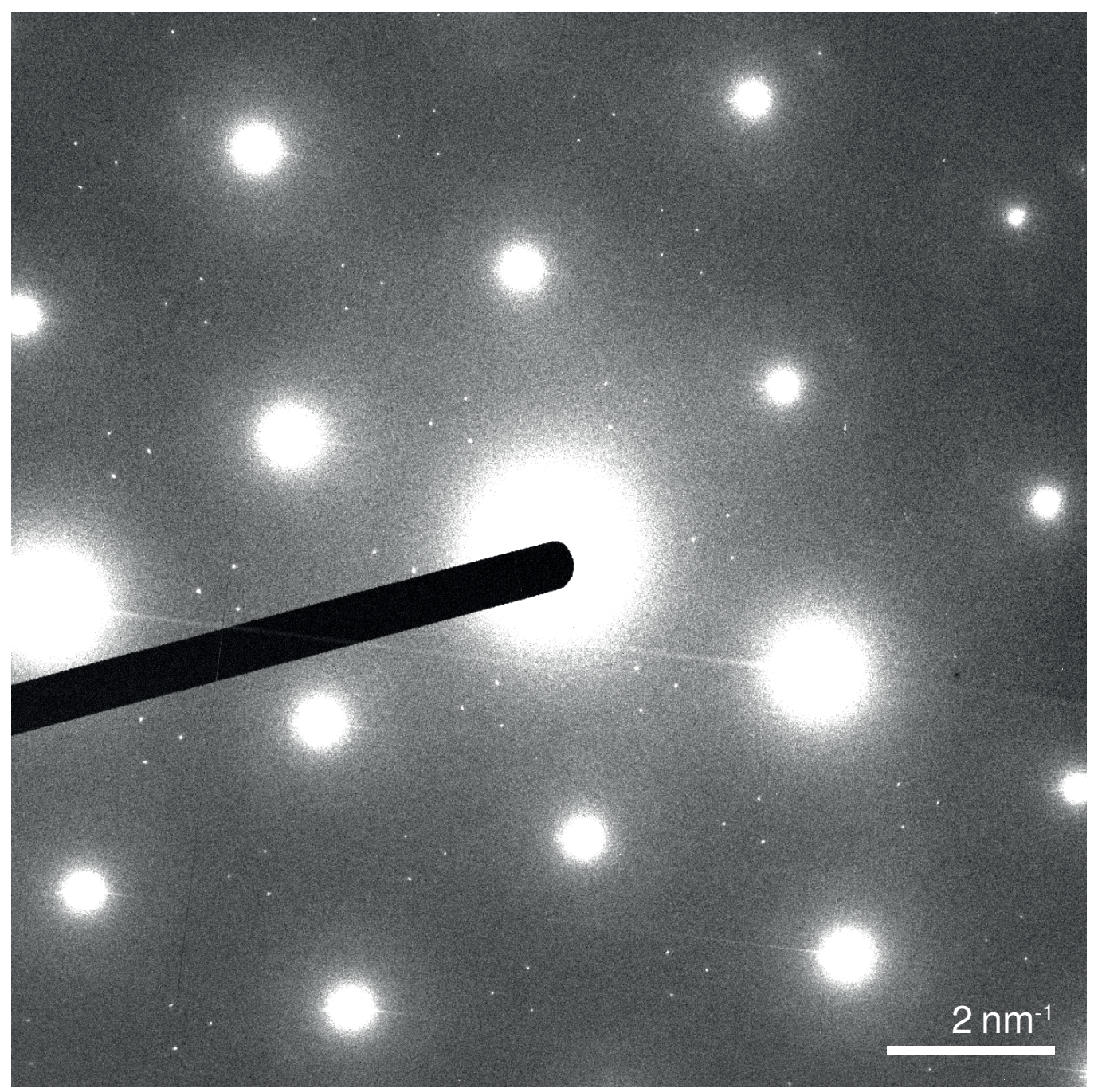

Diffractogram of the IC phase in $1 T-\mathrm{TaS}_{2}$ as shown in Fig. 5.5. 



\section{Bibliography}

1. S. J. Moniz, S. A. Shevlin, D. J. Martin, Z. X. Guo, J. Tang, Visible-light driven heterojunction photocatalysts for water splitting - a critical review. Energy Environ. Sci. 8, 731-759 (2015); doi:10.1039/c4ee03271c (cit. on p. 1).

2. K. Sivula, R. van de Krol, Semiconducting materials for photoelectrochemical energy conversion. Nat. Rev. Mater. 1, 15010 (2016); doi:10.1038/natrevmats.2015.10 (cit. on pp. 1, 68).

3. M. A. Green, S. P. Bremner, Energy conversion approaches and materials for highefficiency photovoltaics. Nat. Mater. 16, 23-34 (2016); doi:10.1038/nmat4676 (cit. on p. 1).

4. F. Wu, J. Maier, Y. Yu, Guidelines and trends for next-generation rechargeable lithium and lithium-ion batteries. Chem. Soc. Rev. 49, 1569-1614 (2020); doi:10.1039/C7CS00863E (cit. on pp. 1, 51).

5. J. A. Wilson, A. D. Yoffe, The Transition Metal Dichalcogenides: Discussion and Interpretation of the Observed Optical, Electrical and Structural Properties. Adv. Phys. 18, 193-335 (1969); doi:10.1080/00018736900101307 (cit. on pp. 1, 30, 56, 58).

6. Q. H. Wang, K. Kalantar-Zadeh, A. Kis, J. N. Coleman, M. S. Strano, Electronics and optoelectronics of two-dimensional transition metal dichalcogenides. Nat. Nanotechnol. 7, 699-712 (2012); doi:10.1038/nnano.2012.193 (cit. on pp. 1, 29, 31, 56).

7. R. Ang, Y. Tanaka, E. Ieki, K. Nakayama, T. Sato, L. J. Li, W. J. Lu, Y. P. Sun, T. Takahashi, Real-Space Coexistence of the Melted Mott State and Superconductivity in Fe-Substituted $1 T-\mathrm{TaS}_{2}$. Phys. Rev. Lett. 109, 176403 (2012); doi:10.1103/PhysRevLett.109.176403 (cit. on pp. 1, 43). 
8. X. M. Chen, A. J. Miller, C. Nugroho, G. A. de la Peña, Y. I. Joe, A. Kogar, J. D. Brock, J. Geck, G. J. MacDougall, S. L. Cooper, E. Fradkin, D. J. Van Harlingen, P. Abbamonte, Influence of Ti doping on the incommensurate charge density wave in $1 T-\mathrm{TaS}_{2}$. Phys. Rev. B 91, 245113 (2015); doi:10.1103/PhysRevB.91.245113 (cit. on pp. 1, 43).

9. A. W. Tsen, R. Hovden, D. Wang, Y. D. Kim, J. Okamoto, K. A. Spoth, Y. Liu, W. Lu, Y. Sun, J. C. Hone, L. F. Kourkoutis, P. Kim, A. N. Pasupathy, Structure and control of charge density waves in two-dimensional $1 \mathrm{~T}^{-T a S_{2}}$. Proc. Natl. Acad. Sci. U.S.A. 112, 15054-15059 (2015); doi:10.1073/pnas.1512092112 (cit. on pp. 1, 43, 112).

10. E. J. Sie, C. M. Nyby, C. D. Pemmaraju, S. J. Park, X. Shen, J. Yang, M. C. Hoffmann, B. K. Ofori-Okai, R. Li, A. H. Reid, S. Weathersby, E. Mannebach, N. Finney, D. Rhodes, D. Chenet, A. Antony, L. Balicas, J. Hone, T. P. Devereaux, T. F. Heinz, X. Wang, A. M. Lindenberg, An ultrafast symmetry switch in a Weyl semimetal. Nature 565, 61-66 (2019); doi:10.1038/s41586-018-0809-4 (cit. on pp. 1-3, 5, 68).

11. M. Rajapakse, B. Karki, U. O. Abu, S. Pishgar, M. R. K. Musa, S. M. S. Riyadh, M. Yu, G. Sumanasekera, J. B. Jasinski, Intercalation as a versatile tool for fabrication, property tuning, and phase transitions in 2D materials. npj 2D Mater. Appl. 5, 30 (2021); doi:10.1038/s41699-021-00211-6 (cit. on pp. 1, 29).

12. J. A. Wilson, F. J. Di Salvo, S. Mahajan, Charge-density waves and superlattices in the metallic layered transition metal dichalcogenides. Adv. Phys. 24, 117-201 (1975); doi:10.1080/00018737500101391 (cit. on pp. 1, 29, 40, 44, 69, 73, 83, 113).

13. J. Ravnik, M. Diego, Y. Gerasimenko, Y. Vaskivskyi, I. Vaskivskyi, T. Mertelj, J. Vodeb, D. Mihailovic, A time-domain phase diagram of metastable states in a charge ordered quantum material. Nat. Commun. 12, 2323 (2021); doi:10.1038/s41467-021-22646-7 (cit. on pp. 2, 44).

14. L. Stojchevska, I. Vaskivskyi, T. Mertelj, P. Kusar, D. Svetin, S. Brazovskii, D. Mihailovic, Ultrafast Switching to a Stable Hidden Quantum State in an Electronic Crystal. Science 344, 177-180 (2014); doi:10.1126/science.1241591 (cit. on pp. 2, 44, 68).

15. D. Cho, S. Cheon, K.-S. Kim, S.-H. Lee, Y.-H. Cho, S.-W. Cheong, H. W. Yeom, Nanoscale manipulation of the Mott insulating state coupled to charge order in $1 T-\mathrm{TaS}_{2}$. Nat. Commun. 7, 10453 (2016); doi:10.1038/ncomms10453 (cit. on pp. 2, 116).

16. L. Ma, C. Ye, Y. Yu, X. F. Lu, X. Niu, S. Kim, D. Feng, D. Tománek, Y.-W. Son, X. H. Chen, Y. Zhang, A metallic mosaic phase and the origin of Mott-insulating state in 1T-TaS 2 . Nat. Commun. 7, 10956 (2016); doi:10.1038/ncomms10956 (cit. on pp. 2, 116). 
17. Y. A. Gerasimenko, I. Vaskivskyi, M. Litskevich, J. Ravnik, J. Vodeb, M. Diego, V. Kabanov, D. Mihailovic, Quantum jamming transition to a correlated electron glass in 1T-TaS ${ }_{2}$. Nat. Mater. 18, 1078-1083 (2019); doi:10.1038/s41563-019-0423-3 (cit. on p. 2).

18. W. Li, X. Qian, J. Li, Phase transitions in 2D materials. Nat. Rev. Mater. (2021); doi:10.1 038/s41578-021-00304-0 (cit. on p. 2).

19. A. de la Torre, D. M. Kennes, M. Claassen, S. Gerber, J. W. McIver, M. A. Sentef, Nonthermal pathways to ultrafast control in quantum materials. arXiv (2021); arXiv:2103 $.14888 \mathrm{v} 1$ [cond-mat.str-el] (cit. on p. 2).

20. M. Rini, R. Tobey, N. Dean, J. Itatani, Y. Tomioka, Y. Tokura, R. W. Schoenlein, A. Cavalleri, Control of the electronic phase of a manganite by mode-selective vibrational excitation. Nature 449, 72-74 (2007); doi:10.1038/nature06119 (cit. on p. 2).

21. P. Beaud, A. Caviezel, S. O. Mariager, L. Rettig, G. Ingold, C. Dornes, S. W. Huang, J. A. Johnson, M. Radovic, T. Huber, T. Kubacka, A. Ferrer, H. T. Lemke, M. Chollet, D. Zhu, J. M. Glownia, M. Sikorski, A. Robert, H. Wadati, M. Nakamura, M. Kawasaki, Y. Tokura, S. L. Johnson, U. Staub, A time-dependent order parameter for ultrafast photoinduced phase transitions. Nat. Mater. 13, 923-927 (2014); doi:10.1038/nmat4046 (cit. on pp. 2, 122).

22. M. Mitrano, A. Cantaluppi, D. Nicoletti, S. Kaiser, A. Perucchi, S. Lupi, P. Di Pietro, D. Pontiroli, M. Riccò, S. R. Clark, D. Jaksch, A. Cavalleri, Possible light-induced superconductivity in $\mathrm{K}_{3} \mathrm{C}_{60}$ at high temperature. Nature 530, 461-464 (2016); doi:10.10 38/nature16522 (cit. on p. 2).

23. Z. Ye, D. Sun, T. F. Heinz, Optical manipulation of valley pseudospin. Nat. Phys. 13, 26-29 (2017); doi:10.1038/nphys3891 (cit. on pp. 2, 31).

24. M. R. Otto, L. P. René de Cotret, D. A. Valverde-Chavez, K. L. Tiwari, N. Émond, M. Chaker, D. G. Cooke, B. J. Siwick, How optical excitation controls the structure and properties of vanadium dioxide. Proc. Natl. Acad. Sci. U.S.A. 116, 450-455 (2019); doi:10.1073/pnas.1808414115 (cit. on pp. 2, 5, 24, 105, 121, 122).

25. J. G. Horstmann, H. Böckmann, B. Wit, F. Kurtz, G. Storeck, C. Ropers, Coherent control of a surface structural phase transition. Nature 583, 232-236 (2020); doi:10.1038/s41586 -020-2440-4 (cit. on pp. 2, 5). 
26. A. Kogar, A. Zong, P. E. Dolgirev, X. Shen, J. Straquadine, Y.-Q. Bie, X. Wang, T. Rohwer, I.-C. Tung, Y. Yang, R. Li, J. Yang, S. Weathersby, S. Park, M. E. Kozina, E. J. Sie, H. Wen, P. Jarillo-Herrero, I. R. Fisher, X. Wang, N. Gedik, Light-induced charge density wave in $\mathrm{LaTe}_{3}$. Nat. Phys. 16, 159-163 (2020); doi:10.1038/s41567-019-0705-3 (cit. on pp. 2, 3, 5, 39, 68, 122).

27. B. Yan, C. Felser, Topological Materials: Weyl Semimetals. Annu. Rev. Condens. Matter Phys. 8, 337-354 (2017); doi:10.1146/annurev-conmatphys-031016-025458 (cit. on p. 2).

28. S. Ji, O. Grånäs, J. Weissenrieder, Manipulation of Stacking Order in $T d-\mathrm{WTe}_{2}$ by Ultrafast Optical Excitation. ACS Nano 15, 8826-8835 (2021); doi:10.1021/acsnano.1c01301 (cit. on pp. 2, 24).

29. F. Zhou, J. Williams, S. Sun, C. D. Malliakas, M. G. Kanatzidis, A. F. Kemper, C.-Y. Ruan, Nonequilibrium dynamics of spontaneous symmetry breaking into a hidden state of charge-density wave. Nat. Commun. 12, 566 (2021); doi:10.1038/s41467-020-20834-5 (cit. on pp. 2, 122).

30. A. K. Geim, I. V. Grigorieva, Van der Waals heterostructures. Nature 499, 419-425 (2013); doi:10.1038/nature12385 (cit. on pp. 2, 51).

31. S. H. Sung, N. Schnitzer, S. Novakov, I. E. Baggari, X. Luo, J. Gin, N. Vu, Z. Li, T. Brintlinger, Y. Liu, W. Lu, Y. Sun, P. Deotare, K. Sun, L. Zhao, L. F. Kourkoutis, J. T. Heron, R. Hovden, Two-dimensional charge order stabilized in clean polytype heterostructures. $\operatorname{arXiv}$ (2021); arXiv:2102.09079v1 [cond-mat.mtrl-sci] (cit. on pp. 2, 120).

32. M. K. L. Man, A. Margiolakis, S. Deckoff-Jones, T. Harada, E. L. Wong, M. B. M. Krishna, J. Madéo, A. Winchester, S. Lei, R. Vajtai, P. M. Ajayan, K. M. Dani, Imaging the motion of electrons across semiconductor heterojunctions. Nat. Nanotechnol. 12, 36-40 (2017); doi:10.1038/nnano.2016.183 (cit. on pp. 2, 5, 119).

33. S.-J. Liang, B. Liu, W. Hu, K. Zhou, L. K. Ang, Thermionic Energy Conversion Based on Graphene van der Waals Heterostructures. Sci. Rep. 7, 46211 (2017); doi:10.1038/sre p46211 (cit. on p. 2).

34. C. Li, Q. Cao, F. Wang, Y. Xiao, Y. Li, J.-J. Delaunay, H. Zhu, Engineering graphene and TMDs based van der Waals heterostructures for photovoltaic and photoelectrochemical solar energy conversion. Chem. Soc. Rev. 47, 4981-5037 (2018); doi:10.1039/C8CS0006 $7 \mathrm{~K}$ (cit. on p. 2). 
35. S. Aeschlimann, A. Rossi, M. Chávez-Cervantes, R. Krause, B. Arnoldi, B. Stadtmüller, M. Aeschlimann, S. Forti, F. Fabbri, C. Coletti, I. Gierz, Direct evidence for efficient ultrafast charge separation in epitaxial $\mathrm{WS}_{2}$ /graphene heterostructures. Sci. Adv. 6, eaay0761 (2020); doi:10.1126/sciadv.aay0761 (cit. on pp. 2, 5).

36. E. Bauer, "LEEM, SPLEEM and SPELEEM" in Springer Handbook of Microscopy, P. W. Hawkes, J. C. H. Spence, Eds. (Springer, Cham, 2019), pp. 487-535; doi:10.1007/978-3030-00069-1_9 (cit. on pp. 4, 104).

37. J. Feng, A. Scholl, "Photoemission Electron Microscopy" in Springer Handbook of Microscopy, P. W. Hawkes, J. C. H. Spence, Eds. (Springer, Cham, 2019), pp. 537-564; doi:10.1007/978-3-030-00069-1_10 (cit. on p. 4).

38. B. D. Huey, J. Luria, D. A. Bonnell, "Scanning Probe Microscopy in Materials Science" in Springer Handbook of Microscopy, P. W. Hawkes, J. C. H. Spence, Eds. (Springer, Cham, 2019), pp. 1239-1277; doi:10.1007/978-3-030-00069-1_25 (cit. on p. 4).

39. P. Sutter, "Scanning Tunneling Microscopy in Surface Science" in Springer Handbook of Microscopy, P. W. Hawkes, J. C. H. Spence, Eds. (Springer, Cham, 2019), pp. 1331-1368; doi:10.1007/978-3-030-00069-1_27 (cit. on p. 4).

40. T. L. Cocker, V. Jelic, M. Gupta, S. J. Molesky, J. A. J. Burgess, G. D. L. Reyes, L. V. Titova, Y. Y. Tsui, M. R. Freeman, F. A. Hegmann, An ultrafast terahertz scanning tunnelling microscope. Nat. Photonics 7, 620-625 (2013); doi:10.1038/nphoton.2013.151 (cit. on p. 4).

41. T. L. Cocker, D. Peller, P. Yu, J. Repp, R. Huber, Tracking the ultrafast motion of a single molecule by femtosecond orbital imaging. Nature 539, 263-267 (2016); doi:10.1038/nat ure19816 (cit. on p. 4).

42. M. Abdo, S. Sheng, S. Rolf-Pissarczyk, L. Arnhold, J. A. J. Burgess, M. Isobe, L. Malavolti, S. Loth, Variable Repetition Rate THz Source for Ultrafast Scanning Tunneling Microscopy. ACS Photonics 8, 702-708 (2021); doi: 10.1021/acsphotonics.0c01652 (cit. on p. 4).

43. M. A. Huber, M. Plankl, M. Eisele, R. E. Marvel, F. Sandner, T. Korn, C. Schüller, R. F. Haglund Jr., R. Huber, T. L. Cocker, Ultrafast Mid-Infrared Nanoscopy of Strained Vanadium Dioxide Nanobeams. Nano Lett. 16, 1421-1427 (2016); doi:10.1021/acs.nanol ett.5b04988 (cit. on pp. 4, 5, 68, 117, 119). 
44. S. A. Dönges, O. Khatib, B. T. O’Callahan, J. M. Atkin, J. H. Park, D. Cobden, M. B. Raschke, Ultrafast Nanoimaging of the Photoinduced Phase Transition Dynamics in $\mathrm{VO}_{2}$. Nano Lett. 16, 3029-3035 (2016); doi:10.1021/acs.nanolett.5b05313 (cit. on pp. 4, 5, 68, $117,118)$.

45. M. M. Qazilbash, M. Brehm, B.-G. Chae, P.-C. Ho, G. O. Andreev, B.-J. Kim, S. J. Yun, A. V. Balatsky, M. B. Maple, F. Keilmann, H.-T. Kim, D. N. Basov, Mott Transition in $\mathrm{VO}_{2}$ Revealed by Infrared Spectroscopy and Nano-Imaging. Science 318, 1750-1753 (2007); doi:10.1126/science.1150124 (cit. on pp. 5, 121).

46. A. J. Frenzel, A. S. McLeod, D. Z.-R. Wang, Y. Liu, W. Lu, G. Ni, A. W. Tsen, Y. Sun, A. N. Pasupathy, D. N. Basov, Infrared nanoimaging of the metal-insulator transition in the charge-density-wave van der Waals material $1 T-\mathrm{TaS}_{2}$. Phys. Rev. B 97, 035111 (2018); doi:10.1103/PhysRevB.97.035111 (cit. on pp. 5, 39, 119).

47. F. Schmitt, P. S. Kirchmann, U. Bovensiepen, R. G. Moore, L. Rettig, M. Krenz, J.-H. Chu, N. Ru, L. Perfetti, D. H. Lu, M. Wolf, I. R. Fisher, Z.-X. Shen, Transient Electronic Structure and Melting of a Charge Density Wave in $\mathrm{TbTe}_{3}$. Science 321, 1649-1652 (2008); doi:10.1126/science.1160778 (cit. on p. 5).

48. T. Rohwer, S. Hellmann, M. Wiesenmayer, C. Sohrt, A. Stange, B. Slomski, A. Carr, Y. Liu, L. M. Avila, M. Kalläne, S. Mathias, L. Kipp, K. Rossnagel, M. Bauer, Collapse of long-range charge order tracked by time-resolved photoemission at high momenta. Nature 471, 490-493 (2011); doi:10.1038/nature09829 (cit. on pp. 5, 68).

49. S. Mathias, S. Eich, J. Urbancic, S. Michael, A. V. Carr, S. Emmerich, A. Stange, T. Popmintchev, T. Rohwer, M. Wiesenmayer, A. Ruffing, S. Jakobs, S. Hellmann, P. Matyba, C. Chen, L. Kipp, M. Bauer, H. C. Kapteyn, H. C. Schneider, K. Rossnagel, M. M. Murnane, M. Aeschlimann, Self-amplified photo-induced gap quenching in a correlated electron material. Nat. Commun. 7, 12902 (2016); doi:10.1038/ncomms 12902 (cit. on p. 5).

50. C. W. Nicholson, A. Lücke, W. G. Schmidt, M. Puppin, L. Rettig, R. Ernstorfer, M. Wolf, Beyond the molecular movie: Dynamics of bands and bonds during a photoinduced phase transition. Science 362, 821-825 (2018); doi:10.1126/science.aar4183 (cit. on p. 5).

51. K. Siefermann, A. Neff, "Time-Resolved Photoemission Electron Microscopy" in Encyclopedia of Interfacial Chemistry: Surface Science and Electrochemistry (Elsevier, Amsterdam, 2018), vol. 1, pp. 557-566; doi:10.1016/B978-0-12-409547-2.13270-6 (cit. on pp. 5, 119). 
52. O. Schmidt, M. Bauer, C. Wiemann, R. Porath, M. Scharte, O. Andreyev, G. Schönhense, M. Aeschlimann, Time-resolved two photon photoemission electron microscopy. Appl. Phys. B 74, 223-227 (2002); doi:10.1007/s003400200803 (cit. on p. 5).

53. L. Wang, C. Xu, M.-Y. Li, L.-J. Li, Z.-H. Loh, Unraveling Spatially Heterogeneous Ultrafast Carrier Dynamics of Single-Layer $\mathrm{WSe}_{2}$ by Femtosecond Time-Resolved Photoemission Electron Microscopy. Nano Lett. 18, 5172-5178 (2018); doi:10.1021/acs.nan olett.8b02103 (cit. on pp. 5, 119).

54. M. Dąbrowski, Y. Dai, H. Petek, Ultrafast Photoemission Electron Microscopy: Imaging Plasmons in Space and Time. Chem. Rev. 120, 6247-6287 (2020); doi:10.1021/acs.chem rev.0c00146 (cit. on pp. 5, 119).

55. L. Wittenbecher, E. Viñas Boström, J. Vogelsang, S. Lehman, K. A. Dick, C. Verdozzi, D. Zigmantas, A. Mikkelsen, Unraveling the Ultrafast Hot Electron Dynamics in Semiconductor Nanowires. ACS Nano 15, 1133-1144 (2021); doi:10.1021/acsnano.0c08101 (cit. on pp. 5, 119).

56. O.-H. Kwon, B. Barwick, H. S. Park, J. S. Baskin, A. H. Zewail, Nanoscale Mechanical Drumming Visualized by 4D Electron Microscopy. Nano Lett. 8, 3557-3562 (2008); doi:10.1021/n18029866 (cit. on pp. 5, 24, 25).

57. B. Barwick, H. S. Park, O.-H. Kwon, J. S. Baskin, A. H. Zewail, 4D Imaging of Transient Structures and Morphologies in Ultrafast Electron Microscopy. Science 322, 1227-1231 (2008); doi:10.1126/science.1164000 (cit. on pp. 5, 24, 25).

58. R. M. van der Veen, O.-H. Kwon, A. Tissot, A. Hauser, A. H. Zewail, Single-nanoparticle phase transitions visualized by four-dimensional electron microscopy. Nat. Chem. 5, 395402 (2013); doi:10.1038/nchem.1622 (cit. on pp. 5, 25, 26, 68, 105).

59. D. R. Cremons, D. A. Plemmons, D. J. Flannigan, Defect-mediated phonon dynamics in $\mathrm{TaS}_{2}$ and $\mathrm{WSe}_{2}$. Struct. Dyn. 4, 044019 (2017); doi:10.1063/1.4982817 (cit. on pp. 5, 25).

60. Y. Zhang, D. J. Flannigan, Observation of Anisotropic Strain-Wave Dynamics and FewLayer Dephasing in $\mathrm{MoS}_{2}$ with Ultrafast Electron Microscopy. Nano Lett. 19, 8216-8224 (2019); doi:10.1021/acs.nanolett.9b03596 (cit. on pp. 5, 25, 68).

61. S. A. Reisbick, Y. Zhang, D. J. Flannigan, Influence of Discrete Defects on Observed Acoustic-Phonon Dynamics in Layered Materials Probed with Ultrafast Electron Microscopy. J. Phys. Chem. A 124, 1877-1884 (2020); doi:10.1021/acs.jpca.9b12026 (cit. on pp. 5, 25, 26). 
62. Y.-J. Kim, Y. Lee, K. Kim, O.-H. Kwon, Light-Induced Anisotropic Morphological Dynamics of Black Phosphorus Membranes Visualized by Dark-Field Ultrafast Electron Microscopy. ACS Nano 14, 11383-11393 (2020); doi:10.1021/acsnano.0c03644 (cit. on pp. $5,25,27)$.

63. A. Zong, A. Kogar, N. Gedik, Unconventional light-induced states visualized by ultrafast electron diffraction and microscopy. arXiv (2021); arXiv:2104.10373v2 [cond-mat.mtrlsci] (cit. on p. 5).

64. P. Baum, D.-S. Yang, A. H. Zewail, 4D Visualization of Transitional Structures in Phase Transformations by Electron Diffraction. Science 318, 788-792 (2007); doi:10.1126/scie nce.1147724 (cit. on pp. 5, 24, 68, 121).

65. M. Eichberger, H. Schäfer, M. Krumova, M. Beyer, J. Demsar, H. Berger, G. Moriena, G. Sciaini, R. J. D. Miller, Snapshots of cooperative atomic motions in the optical suppression of charge density waves. Nature 468, 799-802 (2010); doi:10.1038/nature09539 (cit. on pp. 5, 46-48, 57, 68, 69, 71, 73, 81, 101, 105).

66. A. Feist, N. Bach, N. Rubiano da Silva, T. Danz, M. Möller, K. E. Priebe, T. Domröse, J. G. Gatzmann, S. Rost, J. Schauss, S. Strauch, R. Bormann, M. Sivis, S. Schäfer, C. Ropers, Ultrafast transmission electron microscopy using a laser-driven field emitter: Femtosecond resolution with a high coherence electron beam. Ultramicroscopy 176, 63-73 (2017); doi:10.1016/j.ultramic.2016.12.005 (cit. on pp. 5, 8, 21-23, 68, 76, 112, $113,176)$.

67. T. Danz, Q. Liu, X. D. Zhu, L. H. Wang, S. W. Cheong, I. Radu, C. Ropers, R. I. Tobey, Structural and magnetic characterization of large area, free-standing thin films of magnetic ion intercalated dichalcogenides $\mathrm{Mn}_{0.25} \mathrm{TaS}_{2}$ and $\mathrm{Fe}_{0.25} \mathrm{TaS}_{2}$. J. Phys. Condens. Matter 28, 356002 (2016); doi:10.1088/0953-8984/28/35/356002 (cit. on pp. 6, 77, 175).

68. T. Danz, T. Domröse, C. Ropers, Ultrafast nanoimaging of the order parameter in a structural phase transition. Science 371, 371-374 (2021); doi:10.1126/science.abd2774 (cit. on pp. 6, 175).

69. M. Knoll, E. Ruska, Das Elektronenmikroskop. Z. Phys. 78, 318-339 (1932); doi:10.100 7/BF01342199 (cit. on p. 7).

70. E. Ruska, Über Fortschritte im Bau und in der Leistung des magnetischen Elektronenmikroskops. Z. Phys. 87, 580-602 (1934); doi:10.1007/BF01333326 (cit. on p. 7). 
71. J. C. Meyer, S. Kurasch, H. J. Park, V. Skakalova, D. Künzel, A. Groß, A. Chuvilin, G. Algara-Siller, S. Roth, T. Iwasaki, U. Starke, J. H. Smet, U. Kaiser, Experimental analysis of charge redistribution due to chemical bonding by high-resolution transmission electron microscopy. Nat. Mater. 10, 209-215 (2011); doi:10.1038/nmat2941 (cit. on p. 7).

72. V. Moshnyaga, B. Damaschke, O. Shapoval, A. Belenchuk, J. Faupel, O. I. Lebedev, J. Verbeeck, G. van Tendeloo, M. Mücksch, V. Tsurkan, R. Tidecks, K. Samwer, Structural phase transition at the percolation threshold in epitaxial $\left(\mathrm{La}_{0.7} \mathrm{Ca}_{0.3} \mathrm{MnO}_{3}\right)_{1-\mathrm{x}}:(\mathrm{MgO})_{x}$ nanocomposite films. Nat. Mater. 2, 247-252 (2003); doi:10.1038/nmat859 (cit. on p. 7).

73. R. Hovden, A. W. Tsen, P. Liu, B. H. Savitzky, I. El Baggari, Y. Liu, W. Lu, Y. Sun, P. Kim, A. N. Pasupathy, L. F. Kourkoutis, Atomic lattice disorder in charge-density-wave phases of exfoliated dichalcogenides (1T-TaS 2$)$. Proc. Natl. Acad. Sci. U.S.A. 113, 11420-11424 (2016); doi:10.1073/pnas.1606044113 (cit. on pp. 7, 41, 119).

74. G. Lole, V. Roddatis, U. Ross, M. Risch, T. Meyer, L. Rump, J. Geppert, G. Wartner, P. Blöchl, C. Jooss, Dynamic observation of manganese adatom mobility at perovskite oxide catalyst interfaces with water. Commun. Mater. 1, 68 (2020); doi:10.1038/s43246-0 20-00070-6 (cit. on p. 7).

75. E. Ronge, J. Lindner, U. Ross, J. Melder, J. Ohms, V. Roddatis, P. Kurz, C. Jooss, Atom Surface Dynamics of Manganese Oxide under Oxygen Evolution Reaction-Like Conditions Studied by In Situ Environmental Transmission Electron Microscopy. J. Phys. Chem. C 125, 5037-5047 (2021); doi:10.1021/acs.jpcc.0c09806 (cit. on p. 7).

76. N. Shibata, S. D. Findlay, T. Matsumoto, Y. Kohno, T. Seki, G. Sánchez-Santolino, Y. Ikuhara, Direct Visualization of Local Electromagnetic Field Structures by Scanning Transmission Electron Microscopy. Acc. Chem. Res. 50, 1502-1512 (2017); doi:10.1021 /acs.accounts.7b00123 (cit. on pp. 7, 103, 120).

77. R. F. Egerton, Electron Energy-Loss Spectroscopy in the Electron Microscope (Springer, New York, NY, ed. 3, 2011); doi:10.1007/978-1-4419-9583-4 (cit. on pp. 7, 17, 18, 77, 102).

78. Q. Ding, Y. Zhang, X. Chen, X. Fu, D. Chen, S. Chen, L. Gu, F. Wei, H. Bei, Y. Gao, M. Wen, J. Li, Z. Zhang, T. Zhu, R. O. Ritchie, Q. Yu, Tuning element distribution, structure and properties by composition in high-entropy alloys. Nature 574, 223-227 (2019); doi:10.1038/s41586-019-1617-1 (cit. on p. 7). 
79. D. A. Muller, T. Sorsch, S. Moccio, F. H. Baumann, K. Evans-Lutterodt, G. Timp, The electronic structure at the atomic scale of ultrathin gate oxides. Nature 399, 758-761 (1999); doi:10.1038/21602 (cit. on p. 7).

80. F. S. Hage, G. Radtke, D. M. Kepaptsoglou, M. Lazzeri, Q. M. Ramasse, Single-atom vibrational spectroscopy in the scanning transmission electron microscope. Science $\mathbf{3 6 7}$, 1124-1127 (2020); doi:10.1126/science.aba1136 (cit. on p. 7).

81. K. M. Yip, N. Fischer, E. Paknia, A. Chari, H. Stark, Atomic-resolution protein structure determination by cryo-EM. Nature 587, 157-161 (2020); doi:10.1038/s41586-020-2833-4 (cit. on p. 7).

82. E. Callaway, Revolutionary cryo-EM is taking over structural biology. Nature 578, 201 (2020); doi:10.1038/d41586-020-00341-9 (cit. on p. 7).

83. A. H. Zewail, Four-Dimensional Electron Microscopy. Science 328, 187-193 (2010); doi:10.1126/science.1166135 (cit. on pp. 7, 21).

84. G. C. Cho, W. Kütt, H. Kurz, Subpicosecond time-resolved coherent-phonon oscillations in GaAs. Phys. Rev. Lett. 65, 764-766 (1990); doi:10.1103/PhysRevLett.65.764 (cit. on p. 7).

85. A. H. Zewail, Femtochemistry: Atomic-Scale Dynamics of the Chemical Bond. J. Phys. Chem. A 104, 5660-5694 (2000); doi:10.1021/jp001460h (cit. on p. 7).

86. S. Mukamel, Multidimensional Femtosecond Correlation Spectroscopies of Electronic and Vibrational Excitations. Annu. Rev. Phys. Chem. 51, 691-729 (2000); doi:10.1146/an nurev.physchem.51.1.691 (cit. on p. 7).

87. E. Abbe, Beiträge zur Theorie des Mikroskops und der mikroskopischen Wahrnehmung. Archiv f. mikrosk. Anatomie 9, 413-468 (1873); doi:10.1007/BF02956173 (cit. on p. 8).

88. D. B. Williams, C. B. Carter, Transmission Electron Microscopy: A Textbook for Materials Science (Springer, Boston, MA, ed. 2, 2009); doi:10.1007/978-0-387-76501-3 (cit. on pp. $8,10,11,13-15,120)$.

89. R. Erni, M. D. Rossell, C. Kisielowski, U. Dahmen, Atomic-Resolution Imaging with a Sub-50-pm Electron Probe. Phys. Rev. Lett. 102, 096101 (2009); doi:10.1103/PhysRevLe tt.102.096101 (cit. on p. 8). 
90. M. Linck, P. Hartel, S. Uhlemann, F. Kahl, H. Müller, J. Zach, M. Haider, M. Niestadt, M. Bischoff, J. Biskupek, Z. Lee, T. Lehnert, F. Börrnert, H. Rose, U. Kaiser, Chromatic Aberration Correction for Atomic Resolution TEM Imaging from 20 to $80 \mathrm{kV}$. Phys. Rev. Lett. 117, 076101 (2016); doi:10.1103/PhysRevLett.117.076101 (cit. on p. 8).

91. T. Tanigaki, T. Akashi, Y. Takahashi, T. Kawasaki, H. Shinada, "Quest for Ultimate Resolution Using Coherent Electron Waves: An Aberration-Corrected High-Voltage Electron Microscope" in Advances in Imaging and Electron Physics, P. W. Hawkes, Ed. (Academic Press, London, 2016), vol. 198, pp. 69-125; doi:10.1016/bs.aiep.2016.08.004 (cit. on p. 8).

92. F. Börrnert, U. Kaiser, Chromatic- and geometric-aberration-corrected TEM imaging at $80 \mathrm{kV}$ and 20 kV. Phys. Rev. A 98, 023861 (2018); doi:10.1103/PhysRevA.98.023861 (cit. on p. 10).

93. F. Zernike, Das Phasenkontrastverfahren bei der mikroskopischen Beobachtung. Z. techn. Phys. 16, 454-457 (1935) (cit. on p. 11).

94. F. Zernike, How I Discovered Phase Contrast. Science 121, 345-349 (1955); doi:10.1126 /science.121.3141.345 (cit. on p. 11).

95. R. Danev, K. Nagayama, Phase Plates for Transmission Electron Microscopy. Methods Enzymol. 481, 343-369 (2010); doi:10.1016/S0076-6879(10)81014-6 (cit. on pp. 11, 74, 110).

96. H. Lichte, M. Lehmann, Electron holography_basics and applications. Rep. Prog. Phys. 71, 016102 (2008); doi:10.1088/0034-4885/71/1/016102 (cit. on pp. 11, 12).

97. M. De Graef, Introduction to Conventional Transmission Electron Microscopy (Cambridge University Press, Cambridge, 2003); doi:10.1017/CBO9780511615092 (cit. on p. 12).

98. L.-M. Peng, Electron atomic scattering factors and scattering potentials of crystals. Micron 30, 625-648 (1999); doi:10.1016/S0968-4328(99)00033-5 (cit. on pp. 13, 14).

99. C. Colliex, J. M. Cowley, S. L. Dudarev, M. Fink, J. Gjønnes, R. Hilderbrandt, A. Howie, D. F. Lynch, L. M. Peng, G. Ren, A. W. Ross, V. H. Smith, J. C. H. Spence, J. W. Steeds, J. Wang, M. J. Whelan, B. B. Zvyagin, "Electron diffraction" in International Tables for Crystallography, E. Prince, Ed. (International Union of Crystallography, Chester, 2006), vol. C, chap. 4.3, pp. 259-429; doi:10.1107/97809553602060000593 (cit. on pp. 14, 50, 79). 
100. C. A. Volkert, A. M. Minor, Focused Ion Beam Microscopy and Micromachining. MRS Bull. 32, 389-399 (2007); doi:10.1557/mrs2007.62 (cit. on p. 14).

101. T. Meyer, T. Westphal, B. Kressdorf, U. Ross, C. Jooss, M. Seibt, Site-specific plan-view TEM lamella preparation of pristine surfaces with a large field of view. Ultramicroscopy 228, 113320 (2021); doi:10.1016/j.ultramic.2021.113320 (cit. on p. 14).

102. N. Rubiano da Silva, M. Möller, A. Feist, H. Ulrichs, C. Ropers, S. Schäfer, Nanoscale Mapping of Ultrafast Magnetization Dynamics with Femtosecond Lorentz Microscopy. Phys. Rev. X 8, 031052 (2018); doi:10.1103/PhysRevX.8.031052 (cit. on pp. 15, 23, 103, 122).

103. M. Möller, J. H. Gaida, S. Schäfer, C. Ropers, Few-nm tracking of current-driven magnetic vortex orbits using ultrafast Lorentz microscopy. Commun. Phys. 3, 36 (2020); doi:10.10 38/s42005-020-0301-y (cit. on pp. 15, 23, 103).

104. M. Möller, J. H. Gaida, C. Ropers, Pinning and gyration dynamics of magnetic vortices revealed by correlative Lorentz and bright-field imaging. arXiv (2021); arXiv:2107.1020 $8 v 1$ [cond-mat.mes-hall] (cit. on pp. 15, 23, 103).

105. K. S. Novoselov, D. Jiang, F. Schedin, T. J. Booth, V. V. Khotkevich, S. V. Morozov, A. K. Geim, Two-dimensional atomic crystals. Proc. Natl. Acad. Sci. U.S.A. 102, 10451-10453 (2005); doi:10.1073/pnas.0502848102 (cit. on pp. 15, 29, 101).

106. T. F. Malis, D. Steele, Ultramicrotomy for Materials Science. MRS Proc. 199, 3-42 (1990); doi:10.1557/PROC-199-3 (cit. on pp. 16, 57, 58, 100).

107. F. R. Castro-Fernandez, C. M. Sellars, J. A. Whiteman, Measurement of foil thickness and extinction distance by convergent beam transmission electron microscopy. Philos. Mag. A 52, 289-303 (1985); doi:10.1080/01418618508237627 (cit. on p. 17).

108. R. Egerton, S. Cheng, Measurement of local thickness by electron energy-loss spectroscopy. Ultramicroscopy 21, 231-244 (1987); doi:10.1016/0304-3991(87)90148-3 (cit. on p. 17).

109. T. Malis, S. C. Cheng, R. F. Egerton, EELS Log-Ratio Technique for Specimen-Thickness Measurement in the TEM. J. Electron Microsc. Tech. 8, 193-200 (1988); doi:10.1002/je mt.1060080206 (cit. on pp. 18, 59).

110. F. Lenz, Zur Streuung mittelschneller Elektronen in kleinste Winkel. Z. Naturforsch. A 9, 185-204 (1954); doi:10.1515/zna-1954-0301 (cit. on p. 18). 
111. K. Iakoubovskii, K. Mitsuishi, Y. Nakayama, K. Furuya, Thickness Measurements With Electron Energy Loss Spectroscopy. Microsc. Res. Tech. 71, 626-631 (2008); doi:10.100 2/jemt.20597 (cit. on pp. 18, 77).

112. L. Reimer, H. Kohl, Transmission Electron Microscopy: Physics of Image Formation, vol. 36 of Springer Series in Optical Sciences (Springer, New York, ed. 5, 2008); doi:10.1 007/978-0-387-40093-8 (cit. on p. 19).

113. R. Clough, A. Kirkland, "Direct Digital Electron Detectors" in Advances in Imaging and Electron Physics, P. W. Hawkes, Ed. (Academic Press, London, 2016), vol. 198, pp. 1-42; doi:10.1016/bs.aiep.2016.09.001 (cit. on pp. 19, 20).

114. E. R. Fossum, Active Pixel Sensors: Are CCD's Dinosaurs? Proc. SPIE 1900, 2-14 (1993); doi:10.1117/12.148585 (cit. on p. 19).

115. H. R. Tietz, Design and Characterization of 64 MegaPixel Fiber Optic Coupled CMOS Detector for Transmission Electron Microscopy. Microsc. Microanal. 14, 804-805 (2008); doi:10.1017/S1431927608084675 (cit. on p. 19).

116. T. Poikela, J. Plosila, T. Westerlund, M. Campbell, M. D. Gaspari, X. Llopart, V. Gromov, R. Kluit, M. van Beuzekom, F. Zappon, V. Zivkovic, C. Brezina, K. Desch, Y. Fu, A. Kruth, Timepix3: a 65K channel hybrid pixel readout chip with simultaneous ToA/ToT and sparse readout. J. Instrum. 9, C05013 (2014); doi:10.1088/1748-0221/9/05/C05013 (cit. on pp. 19, 20).

117. M. Campbell, J. Alozy, R. Ballabriga, E. Frojdh, E. Heijne, X. Llopart, T. Poikela, L. Tlustos, P. Valerio, W. Wong, Towards a new generation of pixel detector readout chips. $J$. Instrum. 11, C01007 (2016); doi:10.1088/1748-0221/11/01/C01007 (cit. on p. 19).

118. A.-C. Milazzo, P. Leblanc, F. Duttweiler, L. Jin, J. C. Bouwer, S. Peltier, M. Ellisman, F. Bieser, H. S. Matis, H. Wieman, P. Denes, S. Kleinfelder, N.-H. Xuong, Active pixel sensor array as a detector for electron microscopy. Ultramicroscopy 104, 152-159 (2005); doi:10.1016/j.ultramic.2005.03.006 (cit. on p. 19).

119. G. McMullan, A. Faruqi, R. Henderson, "Direct Electron Detectors" in Methods in Enzymology, R. Crowther, Ed. (Academic Press, London, 2016), vol. 579, pp. 1-17; doi:10.1016/bs.mie.2016.05.056 (cit. on p. 20). 
120. A. Arbouet, G. M. Caruso, F. Houdellier, "Ultrafast Transmission Electron Microscopy: Historical Development, Instrumentation, and Applications" in Advances in Imaging and Electron Physics, P. W. Hawkes, Ed. (Academic Press, London, 2018), vol. 207, pp. 1-72; doi:10.1016/bs.aiep.2018.06.001 (cit. on pp. 20, 21).

121. G. H. Campbell, J. T. McKeown, M. K. Santala, "High-Speed Electron Microscopy" in Springer Handbook of Microscopy, P. W. Hawkes, J. C. H. Spence, Eds. (Springer, Cham, 2019), pp. 455-486; doi:10.1007/978-3-030-00069-1_8 (cit. on p. 20).

122. O. Bostanjoglo, R. Liedtke, Tracing fast phase transitions by electron microscopy. Phys. Status Solidi A 60, 451-455 (1980); doi:10.1002/pssa.2210600215 (cit. on p. 20).

123. O. Bostanjoglo, F. Heinricht, Producing high-current nanosecond electron pulses with a standard tungsten hairpin gun. J. Phys. E. Sci. Instrum. 20, 1491-1493 (1987); doi:10.10 88/0022-3735/20/12/014 (cit. on p. 20).

124. H. Dömer, O. Bostanjoglo, High-speed transmission electron microscope. Rev. Sci. Instrum. 74, 4369-4372 (2003); doi:10.1063/1.1611612 (cit. on p. 20).

125. J. S. Kim, T. LaGrange, B. W. Reed, M. L. Taheri, M. R. Armstrong, W. E. King, N. D. Browning, G. H. Campbell, Imaging of Transient Structures Using Nanosecond in Situ TEM. Science 321, 1472-1475 (2008); doi:10.1126/science.1161517 (cit. on p. 20).

126. M. K. Santala, B. W. Reed, S. Raoux, T. Topuria, T. LaGrange, G. H. Campbell, Irreversible reactions studied with nanosecond transmission electron microscopy movies: Laser crystallization of phase change materials. Appl. Phys. Lett. 102, 174105 (2013); doi:10.1063/1.4803921 (cit. on p. 20).

127. T. LaGrange, G. H. Campbell, B. Reed, M. Taheri, J. B. Pesavento, J. S. Kim, N. D. Browning, Nanosecond time-resolved investigations using the in situ of dynamic transmission electron microscope (DTEM). Ultramicroscopy 108, 1441-1449 (2008); doi:10.1016/j.ul tramic.2008.03.013 (cit. on p. 20).

128. T. LaGrange, B. W. Reed, D. J. Masiel, Movie-mode dynamic electron microscopy. MRS Bull. 40, 22-28 (2015); doi:10.1557/mrs.2014.282 (cit. on p. 20).

129. B. W. Reed, A. A. Moghadam, R. S. Bloom, S. T. Park, A. M. Monterrosa, P. M. Price, C. M. Barr, S. A. Briggs, K. Hattar, J. T. McKeown, D. J. Masiel, Electrostatic subframing and compressive-sensing video in transmission electron microscopy. Struct. Dyn. 6, 054303 (2019); doi:10.1063/1.5115162 (cit. on p. 20). 
130. V. A. Lobastov, R. Srinivasan, A. H. Zewail, Four-dimensional ultrafast electron microscopy. Proc. Natl. Acad. Sci. U.S.A. 102, 7069-7073 (2005); doi:10.1073/pnas.05026 07102 (cit. on pp. 21, 64).

131. M. S. Grinolds, V. A. Lobastov, J. Weissenrieder, A. H. Zewail, Four-dimensional ultrafast electron microscopy of phase transitions. Proc. Natl. Acad. Sci. U.S.A. 103, 18427-18431 (2006); doi:10.1073/pnas.0609233103 (cit. on pp. 21, 24, 25, 68, 121).

132. M. Kuwahara, S. Kusunoki, X. G. Jin, T. Nakanishi, Y. Takeda, K. Saitoh, T. Ujihara, H. Asano, N. Tanaka, 30-kV spin-polarized transmission electron microscope with GaAs-GaAsP strained superlattice photocathode. Appl. Phys. Lett. 101, 033102 (2012); doi:10.1063/1.4737177 (cit. on pp. 21, 104).

133. L. Piazza, D. Masiel, T. LaGrange, B. Reed, B. Barwick, F. Carbone, Design and implementation of a fs-resolved transmission electron microscope based on thermionic gun technology. Chem. Phys. 423, 79-84 (2013); doi:10.1016/j.chemphys.2013.06.026 (cit. on p. 21).

134. G. Cao, S. Sun, Z. Li, H. Tian, H. Yang, J. Li, Clocking the anisotropic lattice dynamics of multi-walled carbon nanotubes by four-dimensional ultrafast transmission electron microscopy. Sci. Rep. 5, 8404 (2015); doi:10.1038/srep08404 (cit. on pp. 21, 24).

135. K. Bücker, M. Picher, O. Crégut, T. LaGrange, B. Reed, S. Park, D. Masiel, F. Banhart, Electron beam dynamics in an ultrafast transmission electron microscope with Wehnelt electrode. Ultramicroscopy 171, 8-18 (2016); doi:10.1016/j.ultramic.2016.08.014 (cit. on p. 21).

136. D. A. Plemmons, D. J. Flannigan, Ultrafast electron microscopy: Instrument response from the single-electron to high bunch-charge regimes. Chem. Phys. Lett. 683, 186-192 (2017); doi:10.1016/j.cplett.2017.01.055 (cit. on p. 21).

137. Y. M. Lee, Y. J. Kim, Y.-J. Kim, O.-H. Kwon, Ultrafast electron microscopy integrated with a direct electron detection camera. Struct. Dyn. 4, 044023 (2017); doi:10.1063/1.4983226 (cit. on p. 21).

138. S. Ji, L. Piazza, G. Cao, S. T. Park, B. W. Reed, D. J. Masiel, J. Weissenrieder, Influence of cathode geometry on electron dynamics in an ultrafast electron microscope. Struct. Dyn. 4, 054303 (2017); doi:10.1063/1.4994004 (cit. on p. 21). 
139. J. Williams, F. Zhou, T. Sun, Z. Tao, K. Chang, K. Makino, M. Berz, P. M. Duxbury, C.-Y. Ruan, Active control of bright electron beams with RF optics for femtosecond microscopy. Struct. Dyn. 4, 044035 (2017); doi:10.1063/1.4999456 (cit. on pp. 21, 122).

140. F. Houdellier, G. Caruso, S. Weber, M. Kociak, A. Arbouet, Development of a high brightness ultrafast Transmission Electron Microscope based on a laser-driven cold field emission source. Ultramicroscopy 186, 128-138 (2018); doi:10.1016/j.ultramic.2017.12 .015 (cit. on p. 21).

141. W. Verhoeven, J. van Rens, E. Kieft, P. Mutsaers, O. Luiten, High quality ultrafast transmission electron microscopy using resonant microwave cavities. Ultramicroscopy 188, 85-89 (2018); doi:10.1016/j.ultramic.2018.03.012 (cit. on p. 21).

142. J. Yang, Y. Yoshida, H. Yasuda, Ultrafast electron microscopy with relativistic femtosecond electron pulses. Microscopy 67, 291-295 (2018); doi:10.1093/jmicro/dfy032 (cit. on p. 21).

143. C. Zhu, D. Zheng, H. Wang, M. Zhang, Z. Li, S. Sun, P. Xu, H. Tian, Z. Li, H. Yang, J. Li, Development of analytical ultrafast transmission electron microscopy based on laser-driven Schottky field emission. Ultramicroscopy 209, 112887 (2020); doi:10.1016/j .ultramic.2019.112887 (cit. on p. 21).

144. K. Wang, R. Dahan, M. Shentcis, Y. Kauffmann, A. Ben Hayun, O. Reinhardt, S. Tsesses, I. Kaminer, Coherent interaction between free electrons and a photonic cavity. Nature 582, 50-54 (2020); doi:10.1038/s41586-020-2321-x (cit. on p. 21).

145. O. Zandi, A. E. Sykes, R. D. Cornelius, F. M. Alcorn, B. S. Zerbe, P. M. Duxbury, B. W. Reed, R. M. van der Veen, Transient lensing from a photoemitted electron gas imaged by ultrafast electron microscopy. Nat. Commun. 11, 3001 (2020); doi:10.1038/s41467-020-1 6746-z (cit. on p. 21).

146. P. K. Olshin, M. Drabbels, U. J. Lorenz, Characterization of a time-resolved electron microscope with a Schottky field emission gun. Struct. Dyn. 7, 054304 (2020); doi:10.10 63/4.0000034 (cit. on p. 21).

147. A. Feist, K. E. Echternkamp, J. Schauss, S. V. Yalunin, S. Schäfer, C. Ropers, Quantum coherent optical phase modulation in an ultrafast transmission electron microscope. Nature 521, 200-203 (2015); doi:10.1038/nature14463 (cit. on pp. 21, 23, 64). 
148. A. Feist, S. V. Yalunin, S. Schäfer, C. Ropers, High-purity free-electron momentum states prepared by three-dimensional optical phase modulation. Phys. Rev. Res. 2, 043227 (2020); doi:10.1103/PhysRevResearch.2.043227 (cit. on pp. 21, 23).

149. P. Hommelhoff, C. Kealhofer, M. A. Kasevich, Ultrafast Electron Pulses from a Tungsten Tip Triggered by Low-Power Femtosecond Laser Pulses. Phys. Rev. Lett. 97, 247402 (2006); doi:10.1103/PhysRevLett.97.247402 (cit. on p. 21).

150. C. Ropers, D. R. Solli, C. P. Schulz, C. Lienau, T. Elsaesser, Localized Multiphoton Emission of Femtosecond Electron Pulses from Metal Nanotips. Phys. Rev. Lett. 98, 043907 (2007); doi:10.1103/PhysRevLett.98.043907 (cit. on p. 21).

151. T. Eggebrecht, M. Möller, J. G. Gatzmann, N. Rubiano da Silva, A. Feist, U. Martens, H. Ulrichs, M. Münzenberg, C. Ropers, S. Schäfer, Light-Induced Metastable Magnetic Texture Uncovered by in situ Lorentz Microscopy. Phys. Rev. Lett. 118, 097203 (2017); doi:10.1103/PhysRevLett.118.097203 (cit. on pp. 22, 103).

152. K. E. Echternkamp, A. Feist, S. Schäfer, C. Ropers, Ramsey-type phase control of freeelectron beams. Nat. Phys. 12, 1000-1004 (2016); doi:10.1038/nphys3844 (cit. on p. 23).

153. K. E. Priebe, C. Rathje, S. V. Yalunin, T. Hohage, A. Feist, S. Schäfer, C. Ropers, Attosecond electron pulse trains and quantum state reconstruction in ultrafast transmission electron microscopy. Nat. Photonics 11, 793-797 (2017); doi:10.1038/s41566-017-0045-8 (cit. on p. 23).

154. O. Kfir, H. Lourenço-Martins, G. Storeck, M. Sivis, T. R. Harvey, T. J. Kippenberg, A. Feist, C. Ropers, Controlling free electrons with optical whispering-gallery modes. Nature 582, 46-49 (2020); doi:10.1038/s41586-020-2320-y (cit. on p. 23).

155. B. Barwick, D. J. Flannigan, A. H. Zewail, Photon-induced near-field electron microscopy. Nature 462, 902-906 (2009); doi:10.1038/nature08662 (cit. on p. 23).

156. R. Dahan, A. Gorlach, U. Haeusler, A. Karnieli, O. Eyal, P. Yousefi, M. Segev, A. Arie, G. Eisenstein, P. Hommelhoff, I. Kaminer, Imprinting the quantum statistics of photons on free electrons. arXiv (2021); arXiv:2105.03105v3 [quant-ph] (cit. on p. 23).

157. J.-W. Henke, A. S. Raja, A. Feist, G. Huang, G. Arend, Y. Yang, J. Kappert, R. N. Wang, M. Möller, J. Pan, J. Liu, O. Kfir, C. Ropers, T. J. Kippenberg, Integrated photonics enables continuous-beam electron phase modulation. arXiv (2021); arXiv:2105.03729v2 [physics.optics] (cit. on p. 23). 
158. A. Feist, N. Rubiano da Silva, W. Liang, C. Ropers, S. Schäfer, Nanoscale diffractive probing of strain dynamics in ultrafast transmission electron microscopy. Struct. Dyn. 5, 014302 (2018); doi:10.1063/1.5009822 (cit. on pp. 23, 27, 68, 120).

159. M. Imada, A. Fujimori, Y. Tokura, Metal-insulator transitions. Rev. Mod. Phys. 70, 10391263 (1998); doi:10.1103/RevModPhys.70.1039 (cit. on pp. 24, 35).

160. G. Grüner, Density Waves in Solids (Perseus, Cambridge, MA, 1994); IsBn: 0-7382-0304-1 (cit. on pp. 24, 32-34).

161. F. Carbone, B. Barwick, O.-H. Kwon, H. S. Park, J. Spencer Baskin, A. H. Zewail, EELS femtosecond resolved in 4D ultrafast electron microscopy. Chem. Phys. Lett. 468, 107-111 (2009); doi:10.1016/j.cplett.2008.12.027 (cit. on p. 24).

162. F. Carbone, O.-H. Kwon, A. H. Zewail, Dynamics of Chemical Bonding Mapped by Energy-Resolved 4D Electron Microscopy. Science 325, 181-184 (2009); doi:10.1126/sc ience.1175005 (cit. on p. 24).

163. L. Piazza, C. Ma, H. X. Yang, A. Mann, Y. Zhu, J. Q. Li, F. Carbone, Ultrafast structural and electronic dynamics of the metallic phase in a layered manganite. Struct. Dyn. 1, 014501 (2014); doi:10.1063/1.4835116 (cit. on pp. 24, 68).

164. R. M. van der Veen, T. J. Penfold, A. H. Zewail, Ultrafast core-loss spectroscopy in fourdimensional electron microscopy. Struct. Dyn. 2, 024302 (2015); doi:10.1063/1.4916897 (cit. on p. 24).

165. E. Pomarico, Y.-J. Kim, F. J. García de Abajo, O.-H. Kwon, F. Carbone, R. M. van der Veen, Ultrafast electron energy-loss spectroscopy in transmission electron microscopy. MRS Bull. 43, 497-503 (2018); doi:10.1557/mrs.2018.148 (cit. on p. 24).

166. E. Pomarico, I. Madan, G. Berruto, G. M. Vanacore, K. Wang, I. Kaminer, F. J. García de Abajo, F. Carbone, meV Resolution in Laser-Assisted Energy-Filtered Transmission Electron Microscopy. ACS Photonics 5, 759-764 (2018); doi:10.1021/acsphotonics.7b01 393 (cit. on p. 24).

167. M. Liebtrau, M. Sivis, A. Feist, H. Lourenço-Martins, N. Pazos-Pérez, R. A. AlvarezPuebla, F. J. G. de Abajo, A. Polman, C. Ropers, Spontaneous and stimulated electron-photon interactions in nanoscale plasmonic near fields. Light Sci. Appl. 10, 82 (2021); doi:10.1038/s41377-021-00511-y (cit. on p. 24). 
168. X. Fu, F. Barantani, S. Gargiulo, I. Madan, G. Berruto, T. LaGrange, L. Jin, J. Wu, G. M. Vanacore, F. Carbone, Y. Zhu, Nanoscale-femtosecond dielectric response of Mott insulators captured by two-color near-field ultrafast electron microscopy. Nat. Commun. 11, 5770 (2020); doi:10.1038/s41467-020-19636-6 (cit. on pp. 24, 116, 117, 121).

169. V. A. Lobastov, J. Weissenrieder, J. Tang, A. H. Zewail, Ultrafast Electron Microscopy (UEM): Four-Dimensional Imaging and Diffraction of Nanostructures during Phase Transitions. Nano Lett. 7, 2552-2558 (2007); doi:10.1021/nl071341e (cit. on pp. 24, 121).

170. V. R. Morrison, R. P. Chatelain, K. L. Tiwari, A. Hendaoui, A. Bruhács, M. Chaker, B. J. Siwick, A photoinduced metal-like phase of monoclinic $\mathrm{VO}_{2}$ revealed by ultrafast electron diffraction. Science 346, 445-448 (2014); doi:10.1126/science.1253779 (cit. on pp. 24, 68, 121, 122).

171. D.-S. Yang, P. Baum, A. H. Zewail, Ultrafast electron crystallography of the cooperative reaction path in vanadium dioxide. Struct. Dyn. 3, 034304 (2016); doi:10.1063/1.4953370 (cit. on pp. 24, 121).

172. Z. Tao, F. Zhou, T.-R. T. Han, D. Torres, T. Wang, N. Sepulveda, K. Chang, M. Young, R. R. Lunt, C.-Y. Ruan, The nature of photoinduced phase transition and metastable states in vanadium dioxide. Sci. Rep. 6, 38514 (2016); doi:10.1038/srep38514 (cit. on pp. 24, 121).

173. A. Sood, X. Shen, Y. Shi, S. Kumar, S. J. Park, M. Zajac, Y. Sun, L.-Q. Chen, S. Ramanathan, X. Wang, W. C. Chueh, A. M. Lindenberg, Universal phase dynamics in $\mathrm{VO}_{2}$ switches revealed by ultrafast operando diffraction. Science 373, 352-355 (2021); doi:10.1126/science.abc0652 (cit. on pp. 24, 121).

174. A. Cavalleri, C. Tóth, C. W. Siders, J. A. Squier, F. Ráksi, P. Forget, J. C. Kieffer, Femtosecond Structural Dynamics in $\mathrm{VO}_{2}$ during an Ultrafast Solid-Solid Phase Transition. Phys. Rev. Lett. 87, 237401 (2001); doi:10.1103/PhysRevLett.87.237401 (cit. on pp. 24, 121).

175. S. Wall, S. Yang, L. Vidas, M. Chollet, J. M. Glownia, M. Kozina, T. Katayama, T. Henighan, M. Jiang, T. A. Miller, D. A. Reis, L. A. Boatner, O. Delaire, M. Trigo, Ultrafast disordering of vanadium dimers in photoexcited $\mathrm{VO}_{2}$. Science 362, 572-576 (2018); doi:10.1126/science.aau3873 (cit. on pp. 24, 68, 121).

176. S. Sun, L. Wei, Z. Li, G. Cao, Y. Liu, W. J. Lu, Y. P. Sun, H. Tian, H. Yang, J. Li, Direct observation of an optically induced charge density wave transition in $1 T-\mathrm{TaSe}_{2}$. Phys. Rev. B 92, 224303 (2015); doi:10.1103/PhysRevB.92.224303 (cit. on pp. 24, 57). 
177. L. Wei, S. Sun, C. Guo, Z. Li, K. Sun, Y. Liu, W. Lu, Y. Sun, H. Tian, H. Yang, J. $\mathrm{Li}$, Dynamic diffraction effects and coherent breathing oscillations in ultrafast electron diffraction in layered 1T-TaSeTe. Struct. Dyn. 4, 044012 (2017); doi:10.1063/1.4979643 (cit. on p. 24).

178. S. Ji, O. Grånäs, K. Rossnagel, J. Weissenrieder, Transient three-dimensional structural dynamics in 1T-TaSe 2 . Phys. Rev. B 101, 094303 (2020); doi:10.1103/PhysRevB.101.09 4303 (cit. on p. 24).

179. J. F. Revelli, F. J. DiSalvo, Tantalum Disulfide $\left(\mathrm{TaS}_{2}\right)$ and Its Intercalation Compounds. Inorg. Synth. 19, 35-49 (1979); doi:10.1002/9780470132500.ch8 (cit. on pp. 29, 30, 57).

180. S. Manzeli, D. Ovchinnikov, D. Pasquier, O. V. Yazyev, A. Kis, 2D transition metal dichalcogenides. Nat. Rev. Mater. 2, 17033 (2017); doi:10.1038/natrevmats.2017.33 (cit. on p. 29).

181. W. Y. Liang, "Electronic Properties of Transition Metal Dichalcogenides and Their Intercalation Complexes" in Intercalation in Layered Materials, M. S. Dresselhaus, Ed., vol. 148 of NATO ASI Series—Series B: Physics (Springer, New York, 1986), pp. 31-73; doi:10.1007/978-1-4757-5556-5_2 (cit. on pp. 29, 30, 50-52, 58).

182. A. Spijkerman, J. L. de Boer, A. Meetsma, G. A. Wiegers, S. van Smaalen, X-ray crystalstructure refinement of the nearly commensurate phase of $1 T-\mathrm{TaS}_{2}$ in $(3+2)$-dimensional superspace. Phys. Rev. B 56, 13757-13767 (1997); doi:10.1103/PhysRevB.56.13757 (cit. on pp. 30, 41, 43, 49, 50, 69, 70, 79, 94).

183. A. Meetsma, G. A. Wiegers, R. J. Haange, J. L. de Boer, Structure of $2 \mathrm{H}-\mathrm{TaS}_{2}$. Acta Cryst. C46, 1598-1599 (1990); doi:10.1107/S0108270190000014 (cit. on p. 30).

184. B. Van Laar, H. Rietveld, D. Ijdo, Magnetic and crystallographic structures of $\mathrm{Me}_{x} \mathrm{NbS}_{2}$ and $\mathrm{Me}_{x} \mathrm{TaS}_{2}$. J. Solid State Chem. 3, 154-160 (1971); doi:10.1016/0022-4596(71)90019 -3 (cit. on pp. 30, 52).

185. D. J. Boes, New Solid Lubricants: Their Preparations, Properties and Potential for Aerospace Applications. IEEE Trans. Aerosp. 2, 457-466 (1964); doi:10.1109/TA.1964 .4319624 (cit. on p. 29).

186. B. Sipos, A. F. Kusmartseva, A. Akrap, H. Berger, L. Forró, E. Tutiš, From Mott state to superconductivity in $1 \mathrm{~T}-\mathrm{TaS}_{2}$. Nat. Mater. 7, 960-965 (2008); doi:10.1038/nmat2318 (cit. on p. 29). 
187. M. M. Ugeda, A. J. Bradley, Y. Zhang, S. Onishi, Y. Chen, W. Ruan, C. Ojeda-Aristizabal, H. Ryu, M. T. Edmonds, H.-Z. Tsai, A. Riss, S.-K. Mo, D. Lee, A. Zettl, Z. Hussain, Z.-X. Shen, M. F. Crommie, Characterization of collective ground states in single-layer $\mathrm{NbSe}_{2}$. Nat. Phys. 12, 92-97 (2016); doi:10.1038/nphys3527 (cit. on p. 29).

188. K. S. Novoselov, A. K. Geim, S. V. Morozov, D. Jiang, Y. Zhang, S. V. Dubonos, I. V. Grigorieva, A. A. Firsov, Electric Field Effect in Atomically Thin Carbon Films. Science 306, 666-669 (2004); doi:10.1126/science.1102896 (cit. on p. 29).

189. A. Splendiani, L. Sun, Y. Zhang, T. Li, J. Kim, C.-Y. Chim, G. Galli, F. Wang, Emerging Photoluminescence in Monolayer $\mathrm{MoS}_{2}$. Nano Lett. 10, 1271-1275 (2010); doi:10.1021 /n1903868w (cit. on p. 29).

190. K. F. Mak, C. Lee, J. Hone, J. Shan, T. F. Heinz, Atomically Thin $\mathrm{MoS}_{2}$ : A New DirectGap Semiconductor. Phys. Rev. Lett. 105, 136805 (2010); doi:10.1103/PhysRevLett.105 .136805 (cit. on p. 29).

191. B. Radisavljevic, A. Radenovic, J. Brivio, V. Giacometti, A. Kis, Single-layer $\mathrm{MoS}_{2}$ transistors. Nat. Nanotechnol. 6, 147-150 (2011); doi:10.1038/nnano.2010.279 (cit. on p. 29).

192. D. Jariwala, V. K. Sangwan, L. J. Lauhon, T. J. Marks, M. C. Hersam, Emerging Device Applications for Semiconducting Two-Dimensional Transition Metal Dichalcogenides. ACS Nano 8, 1102-1120 (2014); doi:10.1021/nn500064s (cit. on pp. 29, 31, 56).

193. Z. Y. Zhu, Y. C. Cheng, U. Schwingenschlögl, Giant spin-orbit-induced spin splitting in two-dimensional transition-metal dichalcogenide semiconductors. Phys. Rev. B 84, 153402 (2011); doi:10.1103/PhysRevB.84.153402 (cit. on pp. 31, 56).

194. T. Cao, G. Wang, W. Han, H. Ye, C. Zhu, J. Shi, Q. Niu, P. Tan, E. Wang, B. Liu, J. Feng, Valley-selective circular dichroism of monolayer molybdenum disulphide. Nat. Commun. 3, 887 (2012); doi:10.1038/ncomms1882 (cit. on p. 31).

195. H. Zeng, J. Dai, W. Yao, D. Xiao, X. Cui, Valley polarization in $\mathrm{MoS}_{2}$ monolayers by optical pumping. Nat. Nanotechnol. 7, 490-493 (2012); doi:10.1038/nnano.2012.95 (cit. on p. 31).

196. K. F. Mak, K. He, J. Shan, T. F. Heinz, Control of valley polarization in monolayer $\mathrm{MoS}_{2}$ by optical helicity. Nat. Nanotechnol. 7, 494-498 (2012); doi:10.1038/nnano.2012.96 (cit. on p. 31). 
197. T. Danz, A. Neff, J. H. Gaida, R. Bormann, C. Ropers, S. Schäfer, Ultrafast sublattice pseudospin relaxation in graphene probed by polarization-resolved photoluminescence. Phys. Rev. B 95, 241412 (2017); doi:10.1103/PhysRevB.95.241412 (cit. on pp. 31, 176).

198. K. F. Mak, K. L. McGill, J. Park, P. L. McEuen, The valley Hall effect in $\mathrm{MoS}_{2}$ transistors. Science 344, 1489-1492 (2014); doi:10.1126/science.1250140 (cit. on p. 31).

199. X. Xu, W. Yao, D. Xiao, T. F. Heinz, Spin and pseudospins in layered transition metal dichalcogenides. Nat. Phys. 10, 343-350 (2014); doi:10.1038/nphys2942 (cit. on p. 31).

200. R. E. Peierls, Quantum Theory of Solids (Oxford University Press, Oxford, 2001); ISBN: 0-19-850781-X (cit. on p. 32).

201. K. Rossnagel, On the origin of charge-density waves in select layered transition-metal dichalcogenides. J. Phys. Condens. Matter 23, 213001 (2011); doi:10.1088/0953-8984/2 3/21/213001 (cit. on pp. 32, 33, 35, 41, 42, 68, 69).

202. P. Aebi, T. Pillo, H. Berger, F. Lévy, On the search for Fermi surface nesting in quasi-2D materials. J. Electron Spectrosc. Relat. Phenom. 117-118, 433-449 (2001); doi:10.1016 /S0368-2048(01)00262-6 (cit. on p. 34).

203. W. L. McMillan, Landau theory of charge-density waves in transition-metal dichalcogenides. Phys. Rev. B 12, 1187-1196 (1975); doi:10.1103/PhysRevB.12.1187 (cit. on pp. 34, 35, 38, 39).

204. A. W. Overhauser, Observability of Charge-Density Waves by Neutron Diffraction. Phys. Rev. B 3, 3173-3182 (1971); doi:10.1103/PhysRevB.3.3173 (cit. on pp. 34, 35, 70, 74, 78).

205. P. Lee, T. Rice, P. Anderson, Conductivity from charge or spin density waves. Solid State Commun. 14, 703-709 (1974); doi:10.1016/0038-1098(74)90868-0 (cit. on p. 34).

206. R. M. Fleming, C. C. Grimes, Sliding-Mode Conductivity in $\mathrm{NbSe}_{3}$ : Observation of a Threshold Electric Field and Conduction Noise. Phys. Rev. Lett. 42, 1423-1426 (1979); doi:10.1103/PhysRevLett.42.1423 (cit. on p. 34).

207. J. Hubbard, Electron correlations in narrow energy bands. Proc. R. Soc. A 276, 238-257 (1963); doi:10.1098/rspa.1963.0204 (cit. on p. 34).

208. J. González, M. A. Martín-Delgado, G. Sierra, A. H. Vozmediano, “The Mott Transition and the Hubbard Model" in Quantum Electron Liquids and High-T $T_{c}$ Superconductivity, vol. 38 of Lecture Notes in Physics Monographs (Springer, Berlin, 1995), pp. 151-173; doi:10.1007/978-3-540-47678-8_7 (cit. on p. 35). 
209. L. Perfetti, A. Georges, S. Florens, S. Biermann, S. Mitrovic, H. Berger, Y. Tomm, H. Höchst, M. Grioni, Spectroscopic Signatures of a Bandwidth-Controlled Mott Transition at the Surface of $1 T-\mathrm{TaSe}_{2}$. Phys. Rev. Lett. 90, 166401 (2003); doi:10.1103/PhysRevLet t.90.166401 (cit. on p. 35).

210. P. W. Anderson, Absence of Diffusion in Certain Random Lattices. Phys. Rev. 109, 1492 1505 (1958); doi:10.1103/PhysRev.109.1492 (cit. on p. 35).

211. G. E. Andrews, R. Askey, R. Roy, "Bessel Functions and Confluent Hypergeometric Functions" in Special Functions, vol. 71 of Encyclopedia of Mathematics and its Applications (Cambridge University Press, Cambridge, 1999), pp. 187-239; doi:10.1017/CBO978110 7325937.005 (cit. on p. 35).

212. N. Goldenfeld, Lectures on Phase Transitions and the Renormalization Group (CRC Press, Boca Raton, FL, 1992); doi:10.1201/9780429493492 (cit. on pp. 36, 37).

213. A. J. Bray, Theory of phase-ordering kinetics. Adv. Phys. 51, 481-587 (2002); doi:10.108 0/00018730110117433 (cit. on pp. 37, 38).

214. P. C. Hohenberg, B. I. Halperin, Theory of dynamic critical phenomena. Rev. Mod. Phys. 49, 435-479 (1977); doi:10.1103/RevModPhys.49.435 (cit. on pp. 37, 73, 82).

215. E. Ising, Beitrag zur Theorie des Ferromagnetismus. Z. Phys. 31, 253-258 (1925); doi:10 .1007/BF02980577 (cit. on p. 37).

216. L. Onsager, Crystal Statistics. I. A Two-Dimensional Model with an Order-Disorder Transition. Phys. Rev. 65, 117-149 (1944); doi:10.1103/PhysRev.65.117 (cit. on pp. 37, $38)$.

217. J. C. Tolédano, P. Tolédano, The Landau Theory of Phase Transitions, vol. 3 of World Scientific Lecture Notes in Physics (World Scientific, Singapore, 1987); doi:10.1142/0215 (cit. on pp. 38, 82).

218. V. L. Ginzburg, L. D. Landau, "On the Theory of Superconductivity" in Collected Papers of L.D. Landau, D. ter Haar, Ed. (Pergamon Press, Oxford, 1965), pp. 546-568; doi:10.1 016/B978-0-08-010586-4.50078-X (cit. on p. 38).

219. K. Nakanishi, H. Shiba, Domain-like Incommensurate Charge-Density-Wave States and the First-Order Incommensurate-Commensurate Transitions in Layered Tantalum Dichalcogenides. I. 1T-Polytype. J. Phys. Soc. Jpn. 43, 1839-1847 (1977); doi:10.1143 /JPSJ.43.1839 (cit. on pp. 39, 43). 
220. A. Haldar, C. L. Cortes, S. K. Gray, S. Sharifzadeh, P. Darancet, Giant Optomechanical Coupling in the Charge Density Wave State of Tantalum Disulfide. arXiv (2021); arXiv:2 105.08874v2 [cond-mat.mtrl-sci] (cit. on p. 39).

221. R. Shimano, N. Tsuji, Higgs Mode in Superconductors. Annu. Rev. Condens. Matter Phys. 11, 103-124 (2020); doi:10.1146/annurev-conmatphys-031119-050813 (cit. on p. 39).

222. S. Vogelgesang, G. Storeck, J. G. Horstmann, T. Diekmann, M. Sivis, S. Schramm, K. Rossnagel, S. Schäfer, C. Ropers, Phase ordering of charge density waves traced by ultrafast low-energy electron diffraction. Nat. Phys. 14, 184-190 (2018); doi:10.1038/nphys4309 (cit. on pp. 39, 42, 46, 48, 69, 74).

223. R. Yusupov, T. Mertelj, V. V. Kabanov, S. Brazovskii, P. Kusar, J.-H. Chu, I. R. Fisher, D. Mihailovic, Coherent dynamics of macroscopic electronic order through a symmetry breaking transition. Nat. Phys. 6, 681-684 (2010); doi:10.1038/nphys1738 (cit. on pp. 39, $48,122)$.

224. T. Huber, S. O. Mariager, A. Ferrer, H. Schäfer, J. A. Johnson, S. Grübel, A. Lübcke, L. Huber, T. Kubacka, C. Dornes, C. Laulhe, S. Ravy, G. Ingold, P. Beaud, J. Demsar, S. L. Johnson, Coherent Structural Dynamics of a Prototypical Charge-Density-Wave-to-Metal Transition. Phys. Rev. Lett. 113, 026401 (2014); doi:10.1103/PhysRevLett.113.026401 (cit. on p. 39).

225. M. Trigo, P. Giraldo-Gallo, M. E. Kozina, T. Henighan, M. P. Jiang, H. Liu, J. N. Clark, M. Chollet, J. M. Glownia, D. Zhu, T. Katayama, D. Leuenberger, P. S. Kirchmann, I. R. Fisher, Z. X. Shen, D. A. Reis, Coherent order parameter dynamics in $\mathrm{SmTe}_{3}$. Phys. Rev. B 99, 104111 (2019); doi:10.1103/PhysRevB.99.104111 (cit. on p. 39).

226. M. J. Neugebauer, T. Huber, M. Savoini, E. Abreu, V. Esposito, M. Kubli, L. Rettig, E. Bothschafter, S. Grübel, T. Kubacka, J. Rittmann, G. Ingold, P. Beaud, D. Dominko, J. Demsar, S. L. Johnson, Optical control of vibrational coherence triggered by an ultrafast phase transition. Phys. Rev. B 99, 220302 (2019); doi:10.1103/PhysRevB.99.220302 (cit. on p. 39).

227. M. Trigo, P. Giraldo-Gallo, J. N. Clark, M. E. Kozina, T. Henighan, M. P. Jiang, M. Chollet, I. R. Fisher, J. M. Glownia, T. Katayama, P. S. Kirchmann, D. Leuenberger, H. Liu, D. A. Reis, Z. X. Shen, D. Zhu, Ultrafast formation of domain walls of a charge density wave in SmTe $_{3}$. Phys. Rev. B 103, 054109 (2021); doi:10.1103/PhysRevB.103.054109 (cit. on pp. 39, 122). 
228. O. Y. Gorobtsov, L. Ponet, S. K. K. Patel, N. Hua, A. G. Shabalin, S. Hrkac, J. Wingert, D. Cela, J. M. Glownia, D. Zhu, R. Medapalli, M. Chollet, E. E. Fullerton, S. Artyukhin, O. G. Shpyrko, A. Singer, Femtosecond control of phonon dynamics near a magnetic order critical point. Nat. Commun. 12, 2865 (2021); doi:10.1038/s41467-021-23059-2 (cit. on p. 39).

229. J. Maklar, Y. W. Windsor, C. W. Nicholson, M. Puppin, P. Walmsley, V. Esposito, M. Porer, J. Rittmann, D. Leuenberger, M. Kubli, M. Savoini, E. Abreu, S. L. Johnson, P. Beaud, G. Ingold, U. Staub, I. R. Fisher, R. Ernstorfer, M. Wolf, L. Rettig, Nonequilibrium charge-density-wave order beyond the thermal limit. Nat. Commun. 12, 2499 (2021); doi:10.1038/s41467-021-22778-w (cit. on pp. 39, 122).

230. R. M. A. Lieth, J. C. J. M. Terhell, "Transition Metal Dichalcogenides" in Preparation and Crystal Growth of Materials with Layered Structures, R. M. A. Lieth, Ed., vol. 1 of Physics and Chemistry of Materials with Layered Structures (Springer, Dordrecht, 1977), pp. 141-223; doi:10.1007/978-94-017-2750-1_4 (cit. on p. 40).

231. R. E. Thomson, B. Burk, A. Zettl, J. Clarke, Scanning tunneling microscopy of the chargedensity-wave structure in $1 T-\mathrm{TaS}_{2}$. Phys. Rev. B 49, 16899-16916 (1994); doi:10.1103 /PhysRevB.49.16899 (cit. on pp. 40, 41, 43, 44).

232. F. Jellinek, The system tantalum-sulfur. J. Less Common Met. 4, 9-15 (1962); doi:10.101 6/0022-5088(62)90053-X (cit. on p. 40).

233. C. B. Scruby, P. M. Williams, G. S. Parry, The role of charge density waves in structural transformations of $1 \mathrm{~T} \mathrm{TaS}_{2}$. Philos. Mag. 31, 255-274 (1975); doi:10.1080/1478643750 8228930 (cit. on pp. 40, 41, 44, 69, 70, 86).

234. B. Burk, R. E. Thomson, J. Clarke, A. Zettl, Surface and Bulk Charge Density Wave Structure in 1T-TaS 2 . Science 257, 362-364 (1992); doi:10.1126/science.257.5068.362 (cit. on p. 41).

235. R. Brouwer, F. Jellinek, The low-temperature superstructures of $1 \mathrm{~T}-\mathrm{TaSe}_{2}$ and $2 \mathrm{H}-\mathrm{TaSe}_{2}$. Physica B+C 99, 51-55 (1980); doi:10.1016/0378-4363(80)90209-0 (cit. on p. 41).

236. K. Nakanishi, H. Shiba, Theory of Three-Dimensional Orderings of Charge-Density Waves in 1 T-TaX 2 (X: S, Se). J. Phys. Soc. Jpn. 53, 1103-1113 (1984); doi:10.1143 /JPSJ.53.1103 (cit. on p. 41). 
237. L. Le Guyader, T. Chase, A. H. Reid, R. K. Li, D. Svetin, X. Shen, T. Vecchione, X. J. Wang, D. Mihailovic, H. A. Dürr, Stacking order dynamics in the quasi-two-dimensional dichalcogenide $1 T-\mathrm{TaS}_{2}$ probed with $\mathrm{MeV}$ ultrafast electron diffraction. Struct. Dyn. 4, 044020 (2017); doi:10.1063/1.4982918 (cit. on pp. 41, 46, 105).

238. C. J. Butler, M. Yoshida, T. Hanaguri, Y. Iwasa, Mottness versus unit-cell doubling as the driver of the insulating state in $1 T-\mathrm{TaS}_{2}$. Nat. Commun. 11, 2477 (2020); doi:10.1038/s4 1467-020-16132-9 (cit. on pp. 41, 42).

239. C. Sohrt, A. Stange, M. Bauer, K. Rossnagel, How fast can a Peierls-Mott insulator be melted? Faraday Discuss. 171, 243-257 (2014); doi:10.1039/C4FD00042K (cit. on pp. 42, 44-47, 69).

240. B. Dardel, M. Grioni, D. Malterre, P. Weibel, Y. Baer, F. Lévy, Temperature-dependent pseudogap and electron localization in 1T-TaS 2 . Phys. Rev. B 45, 1462-1465 (1992); doi:10.1103/PhysRevB.45.1462 (cit. on p. 41).

241. L. Perfetti, P. A. Loukakos, M. Lisowski, U. Bovensiepen, H. Berger, S. Biermann, P. S. Cornaglia, A. Georges, M. Wolf, Time Evolution of the Electronic Structure of $1 T-\mathrm{TaS}_{2}$ through the Insulator-Metal Transition. Phys. Rev. Lett. 97, 067402 (2006); doi:10.1103 /PhysRevLett.97.067402 (cit. on pp. 41, 44, 45).

242. L. Perfetti, P. A. Loukakos, M. Lisowski, U. Bovensiepen, M. Wolf, H. Berger, S. Biermann, A. Georges, Femtosecond dynamics of electronic states in the Mott insulator 1T- $\mathrm{TaS}_{2}$ by time resolved photoelectron spectroscopy. New J. Phys. 10, 053019 (2008); doi:10.1088 /1367-2630/10/5/053019 (cit. on pp. 41, 44-46).

243. M. Ligges, I. Avigo, D. Golež, H. U. R. Strand, Y. Beyazit, K. Hanff, F. Diekmann, L. Stojchevska, M. Kalläne, P. Zhou, K. Rossnagel, M. Eckstein, P. Werner, U. Bovensiepen, Ultrafast Doublon Dynamics in Photoexcited 1T-TaS 2 . Phys. Rev. Lett. 120, 166401 (2018); doi:10.1103/PhysRevLett.120.166401 (cit. on pp. 41, 45).

244. A. Simoncig, M. Stupar, B. Ressel, T. Saha, P. Rebernik Ribic, G. De Ninno, Dissecting Mott and charge-density wave dynamics in the photoinduced phase of $1 T-\mathrm{TaS}_{2}$. Phys. Rev. B 103, 155120 (2021); doi:10.1103/PhysRevB.103.155120 (cit. on pp. 41, 45-47).

245. K. T. Law, P. A. Lee, $1 \mathrm{~T}-\mathrm{TaS}_{2}$ as a quantum spin liquid. Proc. Natl. Acad. Sci. U.S.A. 114, 6996-7000 (2017); doi:10.1073/pnas.1706769114 (cit. on p. 42). 
246. T. Ritschel, J. Trinckauf, K. Koepernik, B. Büchner, M. v. Zimmermann, H. Berger, Y. I. Joe, P. Abbamonte, J. Geck, Orbital textures and charge density waves in transition metal dichalcogenides. Nat. Phys. 11, 328-331 (2015); doi:10.1038/nphys3267 (cit. on pp. 42, $68)$.

247. Y. D. Wang, W. L. Yao, Z. M. Xin, T. T. Han, Z. G. Wang, L. Chen, C. Cai, Y. Li, Y. Zhang, Band insulator to Mott insulator transition in $1 T-\mathrm{TaS}_{2}$. Nat. Commun. 11, 4215 (2020); doi:10.1038/s41467-020-18040-4 (cit. on p. 42).

248. D. Shin, N. Tancogne-Dejean, J. Zhang, M. S. Okyay, A. Rubio, N. Park, Identification of the Mott Insulating Charge Density Wave State in $1 \mathrm{~T}-\mathrm{TaS}_{2}$. Phys. Rev. Lett. 126, 196406 (2021); doi:10.1103/PhysRevLett.126.196406 (cit. on p. 42).

249. S. Hellmann, M. Beye, C. Sohrt, T. Rohwer, F. Sorgenfrei, H. Redlin, M. Kalläne, M. Marczynski-Bühlow, F. Hennies, M. Bauer, A. Föhlisch, L. Kipp, W. Wurth, K. Rossnagel, Ultrafast Melting of a Charge-Density Wave in the Mott Insulator 1T-TaS 2 . Phys. Rev. Lett. 105, 187401 (2010); doi:10.1103/PhysRevLett.105.187401 (cit. on pp. 42, 43).

250. G. Storeck, J. G. Horstmann, T. Diekmann, S. Vogelgesang, G. von Witte, S. V. Yalunin, K. Rossnagel, C. Ropers, Structural dynamics of incommensurate charge-density waves tracked by ultrafast low-energy electron diffraction. Struct. Dyn. 7, 034304 (2020); doi:10 $.1063 / 4.0000018$ (cit. on pp. 42, 46, 48, 69, 101).

251. R. Zhao, Y. Wang, D. Deng, X. Luo, W. J. Lu, Y.-P. Sun, Z.-K. Liu, L.-Q. Chen, J. Robinson, Tuning Phase Transitions in $1 \mathrm{~T}-\mathrm{TaS}_{2}$ via the Substrate. Nano Lett. 17, 34713477 (2017); doi:10.1021/acs.nanolett.7b00418 (cit. on p. 43).

252. T. Ritschel, J. Trinckauf, G. Garbarino, M. Hanfland, M. v. Zimmermann, H. Berger, B. Büchner, J. Geck, Pressure dependence of the charge density wave in $1 T$-TaS $\mathrm{S}_{2}$ and its relation to superconductivity. Phys. Rev. B 87, 125135 (2013); doi:10.1103/PhysRevB.87 .125135 (cit. on p. 43).

253. R. Manzke, T. Buslaps, B. Pfalzgraf, M. Skibowski, O. Anderson, On the Phase Transitions in 1T-TaS ${ }_{2}$. Europhys. Lett. 8, 195-200 (1989); doi:10.1209/0295-5075/8/2/015 (cit. on p. 44).

254. P. Fazekas, E. Tosatti, Electrical, structural and magnetic properties of pure and doped 1T-TaS. Philos. Mag. B 39, 229-244 (1979); doi:10.1080/13642817908245359 (cit. on p. 44). 
255. T.-R. T. Han, F. Zhou, C. D. Malliakas, P. M. Duxbury, S. D. Mahanti, M. G. Kanatzidis, C.-Y. Ruan, Exploration of metastability and hidden phases in correlated electron crystals visualized by femtosecond optical doping and electron crystallography. Sci. Adv. 1, e1400173 (2015); doi:10.1126/sciadv.1400173 (cit. on p. 44).

256. C. Laulhé, L. Cario, B. Corraze, E. Janod, T. Huber, G. Lantz, S. Boulfaat, A. Ferrer, S. Mariager, J. Johnson, S. Grübel, A. Lübcke, G. Ingold, P. Beaud, S. Johnson, S. Ravy, $\mathrm{X}$-ray study of femtosecond structural dynamics in the 2D charge density wave compound 1T-TaS 2 . Physica B 460, 100-104 (2015); doi:10.1016/j.physb.2014.11.049 (cit. on pp. 44, 46).

257. I. Vaskivskyi, I. A. Mihailovic, S. Brazovskii, J. Gospodaric, T. Mertelj, D. Svetin, P. Sutar, D. Mihailovic, Fast electronic resistance switching involving hidden charge density wave states. Nat. Commun. 7, 11442 (2016); doi:10.1038/ncomms11442 (cit. on pp. 44, 122).

258. D. Svetin, I. Vaskivskyi, S. Brazovskii, D. Mihailovic, Three-dimensional resistivity and switching between correlated electronic states in $1 \mathrm{~T}-\mathrm{TaS}_{2}$. Sci. Rep. 7, 46048 (2017); doi:10.1038/srep46048 (cit. on pp. 44, 68, 122).

259. K. Sun, S. Sun, C. Zhu, H. Tian, H. Yang, J. Li, Hidden CDW states and insulatorto-metal transition after a pulsed femtosecond laser excitation in layered chalcogenide $1 \mathrm{~T}-\mathrm{TaS}_{2-x} \mathrm{Se}_{x}$. Sci. Adv. 4, eaas9660 (2018); doi:10.1126/sciadv.aas9660 (cit. on p. 44).

260. Y. A. Gerasimenko, P. Karpov, I. Vaskivskyi, S. Brazovskii, D. Mihailovic, Intertwined chiral charge orders and topological stabilization of the light-induced state of a prototypical transition metal dichalcogenide. npj Quantum Mater. 4, 32 (2019); doi:10.1038/s41535-0 19-0172-1 (cit. on p. 44).

261. Q. Stahl, M. Kusch, F. Heinsch, G. Garbarino, N. Kretzschmar, K. Hanff, K. Rossnagel, J. Geck, T. Ritschel, Collapse of layer dimerization in the photo-induced hidden state of 1T-TaS 2 . Nat. Commun. 11, 1247 (2020); doi:10.1038/s41467-020-15079-1 (cit. on p. 44).

262. J. Demsar, L. Forró, H. Berger, D. Mihailovic, Femtosecond snapshots of gap-forming charge-density-wave correlations in quasi-two-dimensional dichalcogenides $1 T-\mathrm{TaS}_{2}$ and 2H-TaSe ${ }_{2}$. Phys. Rev. B 66, 041101 (2002); doi:10.1103/PhysRevB.66.041101 (cit. on pp. 44, 45). 
263. J. C. Petersen, S. Kaiser, N. Dean, A. Simoncig, H. Y. Liu, A. L. Cavalieri, C. Cacho, I. C. E. Turcu, E. Springate, F. Frassetto, L. Poletto, S. S. Dhesi, H. Berger, A. Cavalleri, Clocking the Melting Transition of Charge and Lattice Order in $1 T-\mathrm{TaS}_{2}$ with Ultrafast Extreme-Ultraviolet Angle-Resolved Photoemission Spectroscopy. Phys. Rev. Lett. 107, 177402 (2011); doi:10.1103/PhysRevLett.107.177402 (cit. on pp. 45, 46).

264. S. Hellmann, T. Rohwer, M. Kalläne, K. Hanff, C. Sohrt, A. Stange, A. Carr, M. M. Murnane, H. C. Kapteyn, L. Kipp, M. Bauer, K. Rossnagel, Time-domain classification of charge-density-wave insulators. Nat. Commun. 3, 1069 (2012); doi:10.1038/ncomms2078 (cit. on pp. 45-47, 56).

265. A. Zong, X. Shen, A. Kogar, L. Ye, C. Marks, D. Chowdhury, T. Rohwer, B. Freelon, S. Weathersby, R. Li, J. Yang, J. Checkelsky, X. Wang, N. Gedik, Ultrafast manipulation of mirror domain walls in a charge density wave. Sci. Adv. 4, eaau5501 (2018); doi:10.11 26/sciadv.aau5501 (cit. on pp. 46, 48, 69, 75, 84, 101, 113-116).

266. K. Haupt, M. Eichberger, N. Erasmus, A. Rohwer, J. Demsar, K. Rossnagel, H. Schwoerer, Ultrafast Metamorphosis of a Complex Charge-Density Wave. Phys. Rev. Lett. 116, 016402 (2016); doi:10.1103/PhysRevLett.116.016402 (cit. on pp. 46, 48, 69, 74, 101).

267. C. Laulhé, T. Huber, G. Lantz, A. Ferrer, S. O. Mariager, S. Grübel, J. Rittmann, J. A. Johnson, V. Esposito, A. Lübcke, L. Huber, M. Kubli, M. Savoini, V. L. R. Jacques, L. Cario, B. Corraze, E. Janod, G. Ingold, P. Beaud, S. L. Johnson, S. Ravy, Ultrafast Formation of a Charge Density Wave State in $1 T-\mathrm{TaS}_{2}$ : Observation at Nanometer Scales Using Time-Resolved X-Ray Diffraction. Phys. Rev. Lett. 118, 247401 (2017); doi:10.11 03/PhysRevLett.118.247401 (cit. on pp. 46, 48, 69, 74).

268. A. Zong, A. Kogar, Y.-Q. Bie, T. Rohwer, C. Lee, E. Baldini, E. Ergeçen, M. B. Yilmaz, B. Freelon, E. J. Sie, H. Zhou, J. Straquadine, P. Walmsley, P. E. Dolgirev, A. V. Rozhkov, I. R. Fisher, P. Jarillo-Herrero, B. V. Fine, N. Gedik, Evidence for topological defects in a photoinduced phase transition. Nat. Phys. 15, 27-31 (2019); doi:10.1038/s41567-018-03 11-9 (cit. on pp. 48, 68, 74, 122).

269. G. Storeck, K. Rossnagel, C. Ropers, Ultrafast spot-profile LEED of a charge-density wave phase transition. Appl. Phys. Lett. 118, 221603 (2021); doi:10.1063/5.0052603 (cit. on p. 48).

270. S. S. P. Parkin, R. H. Friend, $3 d$ transition-metal intercalates of the niobium and tantalum dichalcogenides: I. Magnetic properties. Philos. Mag. B 41, 65-93 (1980); doi:10.1080/1 3642818008245370 (cit. on pp. 50, 52, 53, 56-58, 62, 63). 
271. Q. Yun, L. Li, Z. Hu, Q. Lu, B. Chen, H. Zhang, Layered Transition Metal Dichalcogenide-Based Nanomaterials for Electrochemical Energy Storage. Adv. Mater. 32, 1903826 (2020); doi:10.1002/adma.201903826 (cit. on p. 51).

272. D. Wang, L.-M. Liu, S.-J. Zhao, Z.-Y. Hu, H. Liu, Potential Application of Metal Dichalcogenides Double-Layered Heterostructures as Anode Materials for Li-Ion Batteries. J. Phys. Chem. C 120, 4779-4788 (2016); doi:10.1021/acs.jpcc.5b11677 (cit. on p. 51).

273. E. Pomerantseva, Y. Gogotsi, Two-dimensional heterostructures for energy storage. Nat. Energy 2, 17089 (2017); doi:10.1038/nenergy.2017.89 (cit. on p. 51).

274. M. T. Janish, C. B. Carter, In situ TEM observations of the lithiation of molybdenum disulfide. Scr. Mater. 107, 22-25 (2015); doi:10.1016/j.scriptamat.2015.05.011 (cit. on p. 51).

275. W. B. Clark, P. M. Williams, Charge density waves in intercalated $1 \mathrm{~T}-\mathrm{TaS}_{2}$. Philos. Mag. 35, 883-899 (1977); doi:10.1080/14786437708232631 (cit. on p. 51).

276. C. Pettenkofer, W. Jaegermann, Charge-density-wave transformation induced by Na intercalation into $1 T-\mathrm{TaS}_{2}$. Phys. Rev. B 50, 8816-8823 (1994); doi:10.1103/PhysRevB.50.88 16 (cit. on p. 51).

277. Y. Yu, F. Yang, X. F. Lu, Y. J. Yan, Y.-H. Cho, L. Ma, X. Niu, S. Kim, Y.-W. Son, D. Feng, S. Li, S.-W. Cheong, X. H. Chen, Y. Zhang, Gate-tunable phase transitions in thin flakes of 1T-TaS 2 . Nat. Nanotechnol. 10, 270-276 (2015); doi:10.1038/nnano.2014.323 (cit. on p. 51).

278. R. Zhao, B. Grisafe, R. K. Ghosh, K. Wang, S. Datta, J. Robinson, Stabilizing the commensurate charge-density wave in 1T-tantalum disulfide at higher temperatures via potassium intercalation. Nanoscale 11, 6016-6022 (2019); doi :10.1039/C8NR09732A (cit. on p. 51).

279. T. Kim, V. Babenko, B. Novgorodov, D. Kochubey, S. Shaikhutdinov, Destruction of the charge density wave structure in $1 \mathrm{~T}-\mathrm{TaS}_{2}$ under pyridine intercalation. Nucl. Instrum. Methods Phys. Res. A 405, 348-350 (1998); doi:10.1016/S0168-9002(97)00185-X (cit. on p. 51).

280. A. König, K. Koepernik, R. Schuster, R. Kraus, M. Knupfer, B. Büchner, H. Berger, Plasmon evolution and charge-density wave suppression in potassium intercalated $2 \mathrm{H}-\mathrm{TaSe}_{2}$. Europhys. Lett. 100, 27002 (2012); doi:10.1209/0295-5075/100/27002 (cit. on p. 51). 
281. D. Bhoi, S. Khim, W. Nam, B. S. Lee, C. Kim, B.-G. Jeon, B. H. Min, S. Park, K. H. Kim, Interplay of charge density wave and multiband superconductivity in $2 \mathrm{H}-\mathrm{Pd}_{x} \mathrm{TaSe}_{2}$. Sci. Rep. 6, 24068 (2016); doi:10.1038/srep24068 (cit. on p. 51).

282. E. Morosan, H. W. Zandbergen, L. Li, M. Lee, J. G. Checkelsky, M. Heinrich, T. Siegrist, N. P. Ong, R. J. Cava, Sharp switching of the magnetization in $\mathrm{Fe}_{1 / 4} \mathrm{TaS}_{2}$. Phys. Rev. B 75, 104401 (2007); doi:10.1103/PhysRevB.75.104401 (cit. on pp. 52, 58).

283. Z. Dai, Q. Xue, Y. Gong, C. G. Slough, R. V. Coleman, Scanning-probe-microscopy studies of superlattice structures and density-wave structures in $2 \mathrm{H}-\mathrm{NbSe} \mathrm{C}_{2}, 2 \mathrm{H}-\mathrm{TaSe}_{2}$, and 2H-TaS 2 induced by Fe doping. Phys. Rev. B 48, 14543-14555 (1993); doi:10.1103 /PhysRevB.48.14543 (cit. on p. 52).

284. J. M. Vandenberg-Voorhoeve, "Structural and Magnetic Properties of Layered Chalcogenides of the Transition Elements" in Optical and Electrical Properties, P. A. Lee, Ed., vol. 4 of Physics and Chemistry of Materials with Layered Structures (Reidel, Dordrecht, 1976), pp. 423-457; doi:10.1007/978-94-010-1478-6_8 (cit. on pp. 52, 57).

285. H. Hinode, T. Ohtani, M. Wakihara, Homogeneity Range and Some Physical Properties of Intercalation Compounds of $\mathrm{Mn}_{x} \mathrm{TaS}_{2}$. J. Solid State Chem. 114, 1-5 (1995); doi:10.1 006/jssc.1995.1001 (cit. on pp. 52, 57).

286. K.-T. Ko, K. Kim, S. B. Kim, H.-D. Kim, J.-Y. Kim, B. I. Min, J.-H. Park, F.-H. Chang, H.-J. Lin, A. Tanaka, S.-W. Cheong, RKKY Ferromagnetism with Ising-Like Spin States in Intercalated $\mathrm{Fe}_{1 / 4} \mathrm{TaS}_{2}$. Phys. Rev. Lett. 107, 247201 (2011); doi:10.1103/PhysRevLett .107 .247201 (cit. on pp. 52, 58, 62, 104).

287. M. D. Vannette, S. Yeninas, E. Morosan, R. J. Cava, R. Prozorov, Local-moment ferromagnetism and unusual magnetic domains in $\mathrm{Fe}_{1 / 4} \mathrm{TaS}_{2}$ crystals. Phys. Rev. B 80, 024421 (2009); doi:10.1103/PhysRevB.80.024421 (cit. on pp. 52, 53, 58, 63).

288. Y. Ōnuki, K. Ina, T. Hirai, T. Komatsubara, Magnetic Properties of Intercalation Compound: $\mathrm{Mn}_{1 / 4} \mathrm{MX}_{2}$. J. Phys. Soc. Jpn. 55, 347-356 (1986); doi:10.1143/JPSJ.55.347 (cit. on pp. 52, 58).

289. Q. Liu, X. D. Zhu, L. H. Wang, S.-W. Cheong, R. I. Tobey, Ultrafast magnetization and structural dynamics in the intercalated transition metal dichalcogenides $\mathrm{Fe}_{0.25} \mathrm{TaS}_{2}$ and $\mathrm{Mn}_{0.25} \mathrm{TaS}_{2}$. J. Phys. Condens. Matter 28, 194002 (2016); doi:10.1088/0953-8984/28/19 /194002 (cit. on p. 53). 
290. C. Stamm, T. Kachel, N. Pontius, R. Mitzner, T. Quast, K. Holldack, S. Khan, C. Lupulescu, E. F. Aziz, M. Wietstruk, H. A. Dürr, W. Eberhardt, Femtosecond modification of electron localization and transfer of angular momentum in nickel. Nat. Mater. 6, 740-743 (2007); doi:10.1038/nmat1985 (cit. on pp. 53, 68, 103).

291. N. Dean, J. C. Petersen, D. Fausti, R. I. Tobey, S. Kaiser, L. V. Gasparov, H. Berger, A. Cavalleri, Polaronic Conductivity in the Photoinduced Phase of $1 T-\mathrm{TaS}_{2}$. Phys. Rev. Lett. 106, 016401 (2011); doi:10.1103/PhysRevLett.106.016401 (cit. on p. 56).

292. X. Huang, Z. Zeng, H. Zhang, Metal dichalcogenide nanosheets: preparation, properties and applications. Chem. Soc. Rev. 42, 1934-1946 (2013); doi:10.1039/c2cs35387c (cit. on p. 56).

293. P. Zhu, J. Cao, Y. Zhu, J. Geck, Y. Hidaka, S. Pjerov, T. Ritschel, H. Berger, Y. Shen, R. Tobey, J. P. Hill, X. J. Wang, Dynamic separation of electron excitation and lattice heating during the photoinduced melting of the periodic lattice distortion in $2 \mathrm{H}-\mathrm{TaSe}_{2}$. Appl. Phys. Lett. 103, 071914 (2013); doi:10.1063/1.4818460 (cit. on p. 57).

294. E. Möhr-Vorobeva, S. L. Johnson, P. Beaud, U. Staub, R. De Souza, C. Milne, G. Ingold, J. Demsar, H. Schaefer, A. Titov, Nonthermal Melting of a Charge Density Wave in $\mathrm{TiSe}_{2}$. Phys. Rev. Lett. 107, 036403 (2011); doi:10.1103/PhysRevLett.107.036403 (cit. on p. 57).

295. K. Amemiya, H. Kondoh, T. Yokoyama, T. Ohta, A soft X-ray beamline for surface chemistry at the Photon Factory. J. Electron Spectrosc. Relat. Phenom. 124, 151-164 (2002); doi:10.1016/S0368-2048(02)00051-8 (cit. on p. 57).

296. D. Schön, J. Xiao, R. Golnak, M. F. Tesch, B. Winter, J.-J. Velasco-Velez, E. F. Aziz, Introducing Ionic-Current Detection for X-ray Absorption Spectroscopy in Liquid Cells. J. Phys. Chem. Lett. 8, 2087-2092 (2017); doi:10.1021/acs.jpclett.7b00646 (cit. on p. 57).

297. R. Abrudan, F. Brüssing, R. Salikhov, J. Meermann, I. Radu, H. Ryll, F. Radu, H. Zabel, ALICE-An advanced reflectometer for static and dynamic experiments in magnetism at synchrotron radiation facilities. Rev. Sci. Instrum. 86, 063902 (2015); doi:10.1063/1.4921 716 (cit. on pp. 57, 61).

298. R. Nakajima, J. Stöhr, Y. U. Idzerda, Electron-yield saturation effects in $L$-edge x-ray magnetic circular dichroism spectra of Fe, Co, and Ni. Phys. Rev. B 59, 6421-6429 (1999); doi:10.1103/PhysRevB.59.6421 (cit. on pp. 57, 103). 
299. K. Motizuki, N. Suzuki, S. Tomishima, Itinerant magnetism of Mn-intercalated compounds of 2H-type $\mathrm{TaS}_{2}$. J. Magn. Magn. Mater. 104-107, 681-682 (1992); doi:10.1016 /0304-8853(92)90982-T (cit. on pp. 58, 104, 105).

300. Z. Zeng, Z. Yin, X. Huang, H. Li, Q. He, G. Lu, F. Boey, H. Zhang, Single-Layer Semiconducting Nanosheets: High-Yield Preparation and Device Fabrication. Angew. Chem. Int. Ed. 50, 11093-11097 (2011); doi:10.1002/anie.201106004 (cit. on p. 58).

301. J. N. Coleman, M. Lotya, A. O’Neill, S. D. Bergin, P. J. King, U. Khan, K. Young, A. Gaucher, S. De, R. J. Smith, I. V. Shvets, S. K. Arora, G. Stanton, H.-Y. Kim, K. Lee, G. T. Kim, G. S. Duesberg, T. Hallam, J. J. Boland, J. J. Wang, J. F. Donegan, J. C. Grunlan, G. Moriarty, A. Shmeliov, R. J. Nicholls, J. M. Perkins, E. M. Grieveson, K. Theuwissen, D. W. McComb, P. D. Nellist, V. Nicolosi, Two-Dimensional Nanosheets Produced by Liquid Exfoliation of Layered Materials. Science 331, 568-571 (2011); doi:10.1126/science.1194975 (cit. on p. 58).

302. M. Eichberger, M. Krumova, H. Berger, J. Demsar, Sample preparation methods for femtosecond electron diffraction experiments. Ultramicroscopy 127, 9-13 (2013); doi:10 .1016/j.ultramic.2012.07.005 (cit. on pp. 58, 59, 101, 106).

303. Y. J. Choi, S. B. Kim, T. Asada, S. Park, W. Wu, Y. Horibe, S.-W. Cheong, Giant magnetic coercivity and ionic superlattice nano-domains in $\mathrm{Fe}_{0.25} \mathrm{TaS}_{2}$. Europhys. Lett. 86, 37012 (2009); doi:10.1209/0295-5075/86/37012 (cit. on p. 60).

304. W. L. O'Brien, B. P. Tonner, Orbital and spin sum rules in X-ray magnetic circular dichroism. Phys. Rev. B 50, 12672-12681 (1994); doi:10.1103/PhysRevB.50.12672 (cit. on p. 62).

305. C. T. Chen, Y. U. Idzerda, H.-J. Lin, N. V. Smith, G. Meigs, E. Chaban, G. H. Ho, E. Pellegrin, F. Sette, Experimental Confirmation of the X-Ray Magnetic Circular Dichroism Sum Rules for Iron and Cobalt. Phys. Rev. Lett. 75, 152-155 (1995); doi:10.1103/PhysRe vLett.75.152 (cit. on p. 62).

306. T. Kachel, F. Eggenstein, R. Follath, A soft X-ray plane-grating monochromator optimized for elliptical dipole radiation from modern sources. J. Synchrotron Rad. 22, 1301-1305 (2015); doi:10.1107/S1600577515010826 (cit. on p. 62).

307. W. J. Hardy, C.-W. Chen, A. Marcinkova, H. Ji, J. Sinova, D. Natelson, E. Morosan, Very large magnetoresistance in $\mathrm{Fe}_{0.28} \mathrm{TaS}_{2}$ single crystals. Phys. Rev. B 91, 054426 (2015); doi:10.1103/PhysRevB.91.054426 (cit. on p. 63). 
308. I. Radu, K. Vahaplar, C. Stamm, T. Kachel, N. Pontius, H. A. Dürr, T. A. Ostler, J. Barker, R. F. L. Evans, R. W. Chantrell, A. Tsukamoto, A. Itoh, A. Kirilyuk, T. Rasing, A. V. Kimel, Transient ferromagnetic-like state mediating ultrafast reversal of antiferromagnetically coupled spins. Nature 472, 205-208 (2011); doi:10.1038/nature09901 (cit. on p. 64).

309. N. Bergeard, V. López-Flores, V. Halté, M. Hehn, C. Stamm, N. Pontius, E. Beaurepaire, C. Boeglin, Ultrafast angular momentum transfer in multisublattice ferrimagnets. Nat. Commun. 5, 3466 (2014); doi:10.1038/ncomms4466 (cit. on p. 64).

310. H. S. Park, J. S. Baskin, A. H. Zewail, 4D Lorentz Electron Microscopy Imaging: Magnetic Domain Wall Nucleation, Reversal, and Wave Velocity. Nano Lett. 10, 3796-3803 (2010); doi:10.1021/nl102861e (cit. on pp. 64, 103).

311. D. Loke, T. H. Lee, W. J. Wang, L. P. Shi, R. Zhao, Y. C. Yeo, T. C. Chong, S. R. Elliott, Breaking the Speed Limits of Phase-Change Memory. Science 336, 1566-1569 (2012); doi:10.1126/science.1221561 (cit. on p. 68).

312. M. Salinga, E. Carria, A. Kaldenbach, M. Bornhöfft, J. Benke, J. Mayer, M. Wuttig, Measurement of crystal growth velocity in a melt-quenched phase-change material. Nat. Commun. 4, 2371 (2013); doi:10.1038/ncomms3371 (cit. on p. 68).

313. J. Feldmann, N. Youngblood, C. D. Wright, H. Bhaskaran, W. H. P. Pernice, All-optical spiking neurosynaptic networks with self-learning capabilities. Nature 569, 208-214 (2019); doi:10.1038/s41586-019-1157-8 (cit. on p. 68).

314. H. Yang, V. Khayrudinov, V. Dhaka, H. Jiang, A. Autere, H. Lipsanen, Z. Sun, H. Jussila, Nanowire network-based multifunctional all-optical logic gates. Sci. Adv. 4, eaar7954 (2018); doi:10.1126/sciadv.aar7954 (cit. on p. 68).

315. N. A. Spaldin, M. Fiebig, The Renaissance of Magnetoelectric Multiferroics. Science 309, 391-392 (2005); doi:10.1126/science.1113357 (cit. on p. 68).

316. M. Yoshida, R. Suzuki, Y. Zhang, M. Nakano, Y. Iwasa, Memristive phase switching in two-dimensional 1T-TaS 2 crystals. Sci. Adv. 1, e1500606 (2015); doi:10.1126/sciadv.150 0606 (cit. on pp. 68, 122).

317. D. Fausti, R. I. Tobey, N. Dean, S. Kaiser, A. Dienst, M. C. Hoffmann, S. Pyon, T. Takayama, H. Takagi, A. Cavalleri, Light-Induced Superconductivity in a Stripe-Ordered Cuprate. Science 331, 189-191 (2011); doi:10.1126/science.1197294 (cit. on p. 68). 
318. S. M. Falke, C. A. Rozzi, D. Brida, M. Maiuri, M. Amato, E. Sommer, A. De Sio, A. Rubio, G. Cerullo, E. Molinari, C. Lienau, Coherent ultrafast charge transfer in an organic photovoltaic blend. Science 344, 1001-1005 (2014); doi:10.1126/science.1249771 (cit. on p. 68).

319. Materials and methods are available in section 5.2 (cit. on pp. 70-74).

320. J. Verbeeck, H. Tian, P. Schattschneider, Production and application of electron vortex beams. Nature 467, 301-304 (2010); doi:10.1038/nature09366 (cit. on pp. 74, 104).

321. V. Grillo, A. H. Tavabi, F. Venturi, H. Larocque, R. Balboni, G. C. Gazzadi, S. Frabboni, P.-H. Lu, E. Mafakheri, F. Bouchard, R. E. Dunin-Borkowski, R. W. Boyd, M. P. J. Lavery, M. J. Padgett, E. Karimi, Measuring the orbital angular momentum spectrum of an electron beam. Nat. Commun. 8, 15536 (2017); doi:10.1038/ncomms15536 (cit. on p. 74).

322. O. Schwartz, J. J. Axelrod, S. L. Campbell, C. Turnbaugh, R. M. Glaeser, H. Müller, Laser phase plate for transmission electron microscopy. Nat. Methods 16, 1016-1020 (2019); doi:10.1038/s41592-019-0552-2 (cit. on pp. 74, 110).

323. C. Ophus, J. Ciston, J. Pierce, T. R. Harvey, J. Chess, B. J. McMorran, C. Czarnik, H. H. Rose, P. Ercius, Efficient linear phase contrast in scanning transmission electron microscopy with matched illumination and detector interferometry. Nat. Commun. 7, 10719 (2016); doi:10.1038/ncomms10719 (cit. on p. 74).

324. M. J. Stern, L. P. René de Cotret, M. R. Otto, R. P. Chatelain, J.-P. Boisvert, M. Sutton, B. J. Siwick, Mapping momentum-dependent electron-phonon coupling and nonequilibrium phonon dynamics with ultrafast electron diffuse scattering. Phys. Rev. B 97, 165416 (2018); doi:10.1103/PhysRevB.97.165416 (cit. on p. 74).

325. T. Danz, T. Domröse, C. Ropers, Data for: Ultrafast nanoimaging of the order parameter in a structural phase transition. GRO.data (2021); doi:10.25625/6GFN9K (cit. on p. 75).

326. W. M. Haynes, D. R. Lide, T. J. Bruno, Eds., CRC Handbook of Chemistry and Physics (CRC Press, Boca Raton, FL, ed. 97, 2017); ISBN: 978-1-4987-5429-3 (cit. on pp. 77, 95, 108).

327. Norcada Inc., "Silicon Nitride TEM Window Grid Specification” (2017), accessed Nov. 18, 2020; www.norcada.com/wp-content/uploads/2017/03/TEM-Grid-Specsheet-Rev-6.pdf (cit. on pp. 77, 95). 
328. A. Biguri, M. Dosanjh, S. Hancock, M. Soleimani, TIGRE: a MATLAB-GPU toolbox for CBCT image reconstruction. Biomed. Phys. Eng. Express 2, 055010 (2016); doi:10.1 088/2057-1976/2/5/055010 (cit. on p. 77).

329. N. Otsu, A Threshold Selection Method from Gray-Level Histograms. IEEE Trans. Syst. Man Cybern. 9, 62-66 (1979); doi:10.1109/TSMC.1979.4310076 (cit. on p. 78).

330. J. B. W. Webber, A bi-symmetric log transformation for wide-range data. Meas. Sci. Technol. 24, 027001 (2013); doi:10.1088/0957-0233/24/2/027001 (cit. on p. 78).

331. COMSOL Multiphysics v. 5.4 (COMSOL AB, Stockholm, 2018); www.comsol.com (cit. on p. 79).

332. S. Hellmann, C. Sohrt, M. Beye, T. Rohwer, F. Sorgenfrei, M. Marczynski-Bühlow, M. Kalläne, H. Redlin, F. Hennies, M. Bauer, A. Föhlisch, L. Kipp, W. Wurth, K. Rossnagel, Time-resolved x-ray photoelectron spectroscopy at FLASH. New J. Phys. 14, 013062 (2012); doi:10.1088/1367-2630/14/1/013062 (cit. on p. 80).

333. A. Aiyiti, X. Bai, J. Wu, X. Xu, B. Li, Measuring the thermal conductivity and interfacial thermal resistance of suspended $\mathrm{MoS}_{2}$ using electron beam self-heating technique. Sci. Bull. 63, 452-458 (2018); doi:10.1016/j.scib.2018.02.022 (cit. on p. 81).

334. S. F. Meyer, R. E. Howard, G. R. Stewart, J. V. Acrivos, T. H. Geballe, Properties of intercalated $2 \mathrm{H}-\mathrm{NbSe}_{2}, 4 \mathrm{Hb}-\mathrm{TaS}_{2}$, and 1T-TaS 2 . J. Chem. Phys. 62, 4411-4419 (1975); doi:10.1063/1.430342 (cit. on pp. 81, 95).

335. A. Suzuki, M. Koizumi, M. Doyama, Thermal evidences for successive CDW phase transitions in $1 \mathrm{~T}-\mathrm{TaS}_{2}$. Solid State Commun. 53, 201-203 (1985); doi:10.1016/0038-109 8(85)90126-7 (cit. on pp. 81, 83, 95).

336. M. D. Núñez-Regueiro, J. M. Lopez-Castillo, C. Ayache, Thermal Conductivity of $1 T-\mathrm{TaS}_{2}$ and $2 \mathrm{H}-\mathrm{TaSe}_{2}$. Phys. Rev. Lett. 55, 1931-1934 (1985); doi: 10.1103/Phys RevLett.55.1931 (cit. on pp. 81, 95).

337. A. Rettenberger, P. Leiderer, M. Probst, R. Haight, Ultrafast electron transport in layered semiconductors studied with femtosecond-laser photoemission. Phys. Rev. B 56, 1209212095 (1997); doi:10.1103/PhysRevB.56.12092 (cit. on p. 81).

338. P. B. Johnson, R. W. Christy, Optical Constants of the Noble Metals. Phys. Rev. B 6, 4370-4379 (1972); doi:10.1103/PhysRevB.6.4370 (cit. on p. 95). 
339. K. Luke, Y. Okawachi, M. R. E. Lamont, A. L. Gaeta, M. Lipson, Broadband mid-infrared frequency comb generation in a $\mathrm{Si}_{3} \mathrm{~N}_{4}$ microresonator. Opt. Lett. 40, 4823-4826 (2015); doi:10.1364/OL.40.004823 (cit. on p. 95).

340. A. R. Beal, H. P. Hughes, W. Y. Liang, The reflectivity spectra of some group VA transition metal dichalcogenides. J. Phys. C 8, 4236-4248 (1975); doi:10.1088/0022-3719/8/24/015 (cit. on p. 95).

341. A. Jain, K. E. Goodson, Measurement of the Thermal Conductivity and Heat Capacity of Freestanding Shape Memory Thin Films Using the $3 \omega$ Method. J. Heat Transfer 130, 102402 (2008); doi:10.1115/1.2945904 (cit. on pp. 95, 108).

342. J. Mayer, L. A. Giannuzzi, T. Kamino, J. Michael, TEM Sample Preparation and FIBInduced Damage. MRS Bull. 32, 400-407 (2007); doi:10.1557/mrs2007.63 (cit. on pp. 101, 106).

343. M. Pitter, M. B. Hugenschmidt, R. J. Behm, Tantalum deposition on and reaction with the hydrogen terminated diamond $\left(\begin{array}{lll}1 & 0 & 0\end{array}\right)$ surface studied by Auger electron and electron energy loss spectroscopy. Appl. Phys. Lett. 68, 2508-2510 (1996); doi:10.1063/1.115837 (cit. on p. 101).

344. L. Tröger, D. Arvanitis, K. Baberschke, H. Michaelis, U. Grimm, E. Zschech, Full correction of the self-absorption in soft-fluorescence extended x-ray-absorption fine structure. Phys. Rev. B 46, 3283-3289 (1992); doi:10.1103/PhysRevB.46.3283 (cit. on p. 103).

345. B. Buades, A. Picón, E. Berger, I. León, N. Di Palo, S. L. Cousin, C. Cocchi, E. Pellegrin, J. Herrero Martin, S. Mañas-Valero, E. Coronado, T. Danz, C. Draxl, M. Uemoto, K. Yabana, M. Schultze, S. Wall, M. Zürch, J. Biegert, Attosecond state-resolved carrier motion in quantum materials probed by soft x-ray XANES. Appl. Phys. Rev. 8, 011408 (2021); doi:10.1063/5.0020649 (cit. on pp. 103, 176).

346. A. K. Petford-Long, M. De Graef, "Lorentz Microscopy" in Characterization of Materials, E. N. Kaufmann, Ed. (Wiley, Hoboken, NJ, 2012), pp. 1787-1801; doi:10.1002/0471266 965.com137 (cit. on p. 103).

347. T. Wang, D. Zhu, B. Wu, C. Graves, S. Schaffert, T. Rander, L. Müller, B. Vodungbo, C. Baumier, D. P. Bernstein, B. Bräuer, V. Cros, S. de Jong, R. Delaunay, A. Fognini, R. Kukreja, S. Lee, V. López-Flores, J. Mohanty, B. Pfau, H. Popescu, M. Sacchi, A. B. Sardinha, F. Sirotti, P. Zeitoun, M. Messerschmidt, J. J. Turner, W. F. Schlotter, O. Hellwig, R. Mattana, N. Jaouen, F. Fortuna, Y. Acremann, C. Gutt, H. A. Dürr, E. Beaurepaire, C. Boeglin, S. Eisebitt, G. Grübel, J. Lüning, J. Stöhr, A. O. Scherz, Femtosecond Single-Shot 
Imaging of Nanoscale Ferromagnetic Order in Co/Pd Multilayers Using Resonant X-Ray Holography. Phys. Rev. Lett. 108, 267403 (2012); doi:10.1103/PhysRevLett.108.267403 (cit. on p. 103).

348. A. Tripathi, J. Mohanty, S. H. Dietze, O. G. Shpyrko, E. Shipton, E. E. Fullerton, S. S. Kim, I. McNulty, Dichroic coherent diffractive imaging. Proc. Natl. Acad. Sci. U.S.A. 108, 13393-13398 (2011); doi:10.1073/pnas.1104304108 (cit. on p. 103).

349. O. Kfir, S. Zayko, C. Nolte, M. Sivis, M. Möller, B. Hebler, S. S. P. K. Arekapudi, D. Steil, S. Schäfer, M. Albrecht, O. Cohen, S. Mathias, C. Ropers, Nanoscale magnetic imaging using circularly polarized high-harmonic radiation. Sci. Adv. 3, eaao4641 (2017); doi:10.1126/sciadv.aao4641 (cit. on p. 103).

350. S. Zayko, O. Kfir, M. Heigl, M. Lohmann, M. Sivis, M. Albrecht, C. Ropers, Ultrafast high-harmonic nanoscopy of magnetization dynamics. arXiv (2020); arXiv:2011.05450v1 [physics.optics] (cit. on p. 103).

351. P. Schattschneider, S. Rubino, C. Hébert, J. Rusz, J. Kuneš, P. Novák, E. Carlino, M. Fabrizioli, G. Panaccione, G. Rossi, Detection of magnetic circular dichroism using a transmission electron microscope. Nature 441, 486-488 (2006); doi:10.1038/nature04778 (cit. on p. 103).

352. S. Schneider, D. Pohl, S. Löffler, J. Rusz, D. Kasinathan, P. Schattschneider, L. Schultz, B. Rellinghaus, Magnetic properties of single nanomagnets: Electron energy-loss magnetic chiral dichroism on FePt nanoparticles. Ultramicroscopy 171, 186-194 (2016); doi:10.10 16/j.ultramic.2016.09.009 (cit. on p. 104).

353. X. Fu, B. Warot-Fonrose, R. Arras, K. Dumesnil, V. Serin, Quantitative moment study and coupling of $4 f$ rare earth and $3 d$ metal by transmitted electrons. Phys. Rev. B 94, 140416 (2016); doi:10.1103/PhysRevB.94.140416 (cit. on p. 104).

354. J. Rusz, S. Bhowmick, Boundaries for Efficient Use of Electron Vortex Beams to Measure Magnetic Properties. Phys. Rev. Lett. 111, 105504 (2013); doi:10.1103/PhysRevLett.111 .105504 (cit. on p. 104).

355. P. Schattschneider, S. Löffler, M. Stöger-Pollach, J. Verbeeck, Is magnetic chiral dichroism feasible with electron vortices? Ultramicroscopy 136, 81-85 (2014); doi:10.1016/j.ultra mic.2013.07.012 (cit. on p. 104). 
356. N. Rougemaille, A. K. Schmid, Magnetic imaging with spin-polarized low-energy electron microscopy. Eur. Phys. J. Appl. Phys. 50, 20101 (2010); doi:10.1051/epjap/2010048 (cit. on p. 104).

357. A. D. Vu, J. Coraux, G. Chen, A. T. N'Diaye, A. K. Schmid, N. Rougemaille, Unconventional magnetisation texture in graphene/cobalt hybrids. Sci. Rep. 6, 24783 (2016); doi:10.1038/srep24783 (cit. on p. 104).

358. R. Frömter, F. Kloodt, S. Rößler, A. Frauen, P. Staeck, D. R. Cavicchia, L. Bocklage, V. Röbisch, E. Quandt, H. P. Oepen, Time-resolved scanning electron microscopy with polarization analysis. Appl. Phys. Lett. 108, 142401 (2016); doi:10.1063/1.4945053 (cit. on p. 104).

359. C. T. Chantler, Detailed Tabulation of Atomic Form Factors, Photoelectric Absorption and Scattering Cross Section, and Mass Attenuation Coefficients in the Vicinity of $\mathrm{Ab}$ sorption Edges in the Soft X-Ray (Z=30-36, Z=60-89, E=0.1 keV-10 keV), Addressing Convergence Issues of Earlier Work. J. Phys. Chem. Ref. Data 29, 597-1048 (2000); doi:10.1063/1.1321055 (cit. on p. 105).

360. M. Gulde, S. Schweda, G. Storeck, M. Maiti, H. K. Yu, A. M. Wodtke, S. Schafer, C. Ropers, Ultrafast low-energy electron diffraction in transmission resolves polymer/graphene superstructure dynamics. Science 345, 200-204 (2014); doi:10.1126/science.1250658 (cit. on p. 105).

361. I. Utke, P. Hoffmann, J. Melngailis, Gas-assisted focused electron beam and ion beam processing and fabrication. J. Vac. Sci. Technol. B 26, 1197-1276 (2008); doi:10.1116/1 .2955728 (cit. on p. 108).

362. W. Liu, M. Asheghi, Thermal Conductivity Measurements of Ultra-Thin Single Crystal Silicon Layers. J. Heat Transf. 128, 75-83 (2006); doi:10.1115/1.2130403 (cit. on p. 108).

363. A. J. Bullen, K. E. O’Hara, D. G. Cahill, O. Monteiro, A. von Keudell, Thermal conductivity of amorphous carbon thin films. J. Appl. Phys. 88, 6317-6320 (2000); doi:10.1063 /1.1314301 (cit. on p. 108).

364. Diamond Materials GmbH, "The CVD diamond booklet” (2015), accessed Apr. 27, 2021; www.diamond-materials.com/site/assets/files/1095/cvd_diamond_booklet.pdf (cit. on p. 108). 
365. J. Anaya, T. Bai, Y. Wang, C. Li, M. Goorsky, T. Bougher, L. Yates, Z. Cheng, S. Graham, K. Hobart, T. Feygelson, M. Tadjer, T. Anderson, B. Pate, M. Kuball, Simultaneous determination of the lattice thermal conductivity and grain/grain thermal resistance in polycrystalline diamond. Acta Mater. 139, 215-225 (2017); doi:10.1016/j.actamat.2017.0 8.007 (cit. on p. 108).

366. K. Heinemann, H. Poppa, Selected-Zone Dark-Field Electron Microscopy. Appl. Phys. Lett. 20, 122-125 (1972); doi:10.1063/1.1654073 (cit. on p. 110).

367. Z. Wang, A. Fisher, Electron energy-loss spectroscopy of high-angle thermal-diffusescattered electrons in TEM. Ultramicroscopy 48, 183-196 (1993); doi:10.1016/0304-399 1(93)90181-V (cit. on p. 110).

368. S. Bals, B. Kabius, M. Haider, V. Radmilovic, C. Kisielowski, Annular dark field imaging in a TEM. Solid State Commun. 130, 675-680 (2004); doi:10.1016/j.ssc.2004.03.035 (cit. on p. 110).

369. C. Zhang, Q. Xu, P. J. Peters, H. Zandbergen, The use of a central beam stop for contrast enhancement in TEM imaging. Ultramicroscopy 134, 200-206 (2013); doi:10.1016/j.ultr amic.2013.05.019 (cit. on p. 110).

370. R. Danev, B. Buijsse, M. Khoshouei, J. M. Plitzko, W. Baumeister, Volta potential phase plate for in-focus phase contrast transmission electron microscopy. Proc. Natl. Acad. Sci. U.S.A. 111, 15635-15640 (2014); doi:10.1073/pnas.1418377111 (cit. on p. 110).

371. C. Turnbaugh, J. J. Axelrod, S. L. Campbell, J. Y. Dioquino, P. N. Petrov, J. Remis, O. Schwartz, Z. Yu, Y. Cheng, R. M. Glaeser, H. Mueller, High-power near-concentric Fabry-Perot cavity for phase contrast electron microscopy. Rev. Sci. Instrum. 92, 053005 (2021); doi:10.1063/5.0045496 (cit. on p. 110).

372. T. Ishiguro, H. Sato, High-resolution electron microscopy of discommensuration in the nearly commensurate phase on warming of $1 T-\mathrm{TaS}_{2}$. Phys. Rev. B 52, 759-765 (1995); doi:10.1103/PhysRevB.52.759 (cit. on p. 110).

373. Direct Electron, LP, "DE-16 Product Booklet" (2019), accessed Apr. 25, 2021; www.dire ctelectron.com/wp-content/uploads/2019/12/DE-16-Product-Booklet.pdf (cit. on p. 111).

374. M. Reiser, Theory and Design of Charged Particle Beams (Wiley, Weinheim, ed. 2, 2008); doi:10.1002/9783527622047 (cit. on p. 113). 
375. A. Feist, "Next-Generation Ultrafast Transmission Electron Microscopy - Development and Applications,” Dissertation, Georg-August-Universität Göttingen, 2018; hdl.handle.n et/11858/00-1735-0000-002E-E48B-B (cit. on p. 113).

376. S. Vogelgesang, "Ultrafast low-energy electron diffraction at surfaces: Probing transitions and phase-ordering of charge-density waves," Dissertation, Georg-August-Universität Göttingen, 2018; hdl.handle.net/11858/00-1735-0000-002E-E5A1-F (cit. on p. 114).

377. D. A. Plemmons, "Nanoscale Energy Transport Investigated with Ultrafast Electron Microscopy,” Dissertation, University of Minnesota, 2017; hdl.handle.net/11299/191467 (cit. on p. 114).

378. T. Domröse, "Charge Density Wave Dynamics Observed in an Ultrafast Transmission Electron Microscope,” Master's thesis, Georg-August-Universität Göttingen, 2019 (cit. on pp. 115, 176).

379. J. Skolimowski, Y. Gerasimenko, R. Žitko, Mottness collapse without metallization in the domain wall of the triangular-lattice Mott insulator $1 T-\mathrm{TaS}_{2}$. Phys. Rev. Lett. 122, 036802 (2019); doi:10.1103/PhysRevLett.122.036802 (cit. on p. 116).

380. J. W. Park, J. Lee, H. W. Yeom, Zoology of domain walls in quasi-2D correlated charge density wave of 1T-TaS ${ }_{2}$.npj Quantum Mater. 6, 32 (2021); doi:10.1038/s41535-021-00 330-9 (cit. on p. 116).

381. R. Tromp, J. Hannon, A. Ellis, W. Wan, A. Berghaus, O. Schaff, A new aberrationcorrected, energy-filtered LEEM/PEEM instrument. I. Principles and design. Ultramicroscopy 110, 852-861 (2010); doi:10.1016/j.ultramic.2010.03.005 (cit. on p. 119).

382. R. Tromp, J. Hannon, W. Wan, A. Berghaus, O. Schaff, A new aberration-corrected, energy-filtered LEEM/PEEM instrument II. Operation and results. Ultramicroscopy 127, 25-39 (2013); doi:10.1016/j.ultramic.2012.07.016 (cit. on p. 119).

383. K. Medjanik, O. Fedchenko, S. Chernov, D. Kutnyakhov, M. Ellguth, A. Oelsner, B. Schönhense, T. R. F. Peixoto, P. Lutz, C.-H. Min, F. Reinert, S. Däster, Y. Acremann, J. Viefhaus, W. Wurth, H. J. Elmers, G. Schönhense, Direct 3D mapping of the Fermi surface and Fermi velocity. Nat. Mater. 16, 615-621 (2017); doi:10.1038/nmat4875 (cit. on p. 119). 
384. M. Keunecke, C. Möller, D. Schmitt, H. Nolte, G. S. M. Jansen, M. Reutzel, M. Gutberlet, G. Halasi, D. Steil, S. Steil, S. Mathias, Time-resolved momentum microscopy with a $1 \mathrm{MHz}$ high-harmonic extreme ultraviolet beamline. Rev. Sci. Instrum. 91, 063905 (2020); doi:10.1063/5.0006531 (cit. on p. 119).

385. P. D. Nellist, "Scanning Transmission Electron Microscopy" in Springer Handbook of Microscopy, P. W. Hawkes, J. C. H. Spence, Eds. (Springer, Cham, 2019), pp. 49-99; doi:10.1007/978-3-030-00069-1_2 (cit. on p. 119).

386. J. Krehl, G. Guzzinati, J. Schultz, P. Potapov, D. Pohl, J. Martin, J. Verbeeck, A. Fery, B. Büchner, A. Lubk, Spectral field mapping in plasmonic nanostructures with nanometer resolution. Nat. Commun. 9, 4207 (2018); doi:10.1038/s41467-018-06572-9 (cit. on p. 120).

387. C. Ophus, Four-Dimensional Scanning Transmission Electron Microscopy (4D-STEM): From Scanning Nanodiffraction to Ptychography and Beyond. Microsc. Microanal. 25, 563-582 (2019); doi:10.1017/S1431927619000497 (cit. on p. 120).

388. D. Alloyeau, C. Ricolleau, T. Oikawa, C. Langlois, Y. Le Bouar, A. Loiseau, STEM nanodiffraction technique for structural analysis of CoPt nanoparticles. Ultramicroscopy 108, 656-662 (2008); doi:10.1016/j.ultramic.2007.10.006 (cit. on p. 120).

389. Y. Zhu, Z. Cai, P. Chen, Q. Zhang, M. J. Highland, I. W. Jung, D. A. Walko, E. M. Dufresne, J. Jeong, M. G. Samant, S. S. P. Parkin, J. W. Freeland, P. G. Evans, H. Wen, Mesoscopic structural phase progression in photo-excited $\mathrm{VO}_{2}$ revealed by time-resolved x-ray diffraction microscopy. Sci. Rep. 6, 21999 (2016); doi:10.1038/srep21999 (cit. on p. 120).

390. J. D. Budai, J. Hong, M. E. Manley, E. D. Specht, C. W. Li, J. Z. Tischler, D. L. Abernathy, A. H. Said, B. M. Leu, L. A. Boatner, R. J. McQueeney, O. Delaire, Metallization of vanadium dioxide driven by large phonon entropy. Nature 515, 535-539 (2014); doi:10.1 038/nature13865 (cit. on p. 121).

391. L. Vidas, C. M. Günther, T. A. Miller, B. Pfau, D. Perez-Salinas, E. Martínez, M. Schneider, E. Gührs, P. Gargiani, M. Valvidares, R. E. Marvel, K. A. Hallman, R. F. Haglund Jr., S. Eisebitt, S. Wall, Imaging Nanometer Phase Coexistence at Defects During the Insulator-Metal Phase Transformation in $\mathrm{VO}_{2}$ Thin Films by Resonant Soft X-ray Holography. Nano Lett. 18, 3449-3453 (2018); doi:10.1021/acs.nanolett.8b00458 (cit. on p. 121). 
392. A. S. Johnson, J. Valls Conesa, L. Vidas, D. Perez-Salinas, C. M. Günther, B. Pfau, K. A. Hallman, R. F. Haglund Jr., S. Eisebitt, S. Wall, Mapping nanoscale charge states and phase domains with quantitative hyperspectral coherent diffractive imaging spectroscopy. $\operatorname{arXiv}$ (2020); arXiv:2008.11992v1 [physics.optics] (cit. on p. 121).

393. M. Pan, H. Zhong, S. Wang, J. Liu, Z. Li, X. Chen, W. Lu, Properties of $\mathrm{VO}_{2}$ thin film prepared with precursor VO(acac) $)_{2}$. J. Cryst. Growth 265, 121-126 (2004); doi:10.1016 /j.jcrysgro.2003.12.065 (cit. on p. 121).

394. L. Vidas, D. Schick, E. Martínez, D. Perez-Salinas, A. Ramos-Álvarez, S. Cichy, S. Batlle-Porro, A. S. Johnson, K. A. Hallman, R. F. Haglund Jr., S. Wall, Does $\mathrm{VO}_{2}$ Host a Transient Monoclinic Metallic Phase? Phys. Rev. X 10, 031047 (2020); doi:10.1103/Phys RevX.10.031047 (cit. on p. 122).

395. F. J. Di Salvo, D. E. Moncton, J. V. Waszczak, Electronic properties and superlattice formation in the semimetal TiSe 2 . Phys. Rev. B 14, 4321-4328 (1976); doi:10.1103/Phys RevB.14.4321 (cit. on p. 122).

396. L. Rettig, R. Cortés, J.-H. Chu, I. R. Fisher, F. Schmitt, R. G. Moore, Z.-X. Shen, P. S. Kirchmann, M. Wolf, U. Bovensiepen, Persistent order due to transiently enhanced nesting in an electronically excited charge density wave. Nat. Commun. 7, 10459 (2016); doi:10.1 038/ncomms10459 (cit. on p. 122).

397. B. Kressdorf, T. Meyer, M. ten Brink, C. Seick, S. Melles, N. Ottinger, T. Titze, H. Meer, A. Weisser, J. Hoffmann, S. Mathias, H. Ulrichs, D. Steil, M. Seibt, P. E. Blöchl, C. Jooss, Orbital-order phase transition in $\operatorname{Pr}_{1-x} \mathrm{Ca}_{x} \mathrm{MnO}_{3}$ probed by photovoltaics. Phys. Rev. $B$ 103, 235122 (2021); doi:10.1103/PhysRevB.103.235122 (cit. on p. 122).

398. M. J. Hollander, Y. Liu, W.-J. Lu, L.-J. Li, Y.-P. Sun, J. A. Robinson, S. Datta, Electrically Driven Reversible Insulator-Metal Phase Transition in $1 \mathrm{~T}-\mathrm{TaS}_{2}$. Nano Lett. 15, 18611866 (2015); doi:10.1021/nl504662b (cit. on p. 122).

399. K. Jhuria, J. Hohlfeld, A. Pattabi, E. Martin, A. Y. Arriola Córdova, X. Shi, R. Lo Conte, S. Petit-Watelot, J. C. Rojas-Sanchez, G. Malinowski, S. Mangin, A. Lemaître, M. Hehn, J. Bokor, R. B. Wilson, J. Gorchon, Spin-orbit torque switching of a ferromagnet with picosecond electrical pulses. Nat. Electron. 3, 680-686 (2020); doi:10.1038/s41928-02000488-3 (cit. on p. 122).

400. L. Zhang, J. P. Hoogenboom, B. Cook, P. Kruit, Photoemission sources and beam blankers for ultrafast electron microscopy. Struct. Dyn. 6, 051501 (2019); doi:10.1063/1.5117058 (cit. on p. 122). 
401. T. van Oudheusden, E. F. de Jong, S. B. van der Geer, W. P. E. M. Op 't Root, O. J. Luiten, B. J. Siwick, Electron source concept for single-shot sub-100 fs electron diffraction in the $100 \mathrm{keV}$ range. J. Appl. Phys. 102, 093501 (2007); doi:10.1063/1.2801027 (cit. on p. 122).

402. J. Maxson, D. Cesar, G. Calmasini, A. Ody, P. Musumeci, D. Alesini, Direct Measurement of Sub-10 fs Relativistic Electron Beams with Ultralow Emittance. Phys. Rev. Lett. 118, 154802 (2017); doi:10.1103/PhysRevLett.118.154802 (cit. on p. 122).

403. M. R. Otto, L. P. René de Cotret, M. J. Stern, B. J. Siwick, Solving the jitter problem in microwave compressed ultrafast electron diffraction instruments: Robust sub-50 fs cavity-laser phase stabilization. Struct. Dyn. 4, 051101 (2017); doi:10.1063/1.4989960 (cit. on p. 122).

404. C. Kealhofer, W. Schneider, D. Ehberger, A. Ryabov, F. Krausz, P. Baum, All-optical control and metrology of electron pulses. Science 352, 429-433 (2016); doi:10.1126/scie nce.aae0003 (cit. on p. 122).

405. D. Zhang, A. Fallahi, M. Hemmer, X. Wu, M. Fakhari, Y. Hua, H. Cankaya, A.-L. Calendron, L. E. Zapata, N. H. Matlis, F. X. Kärtner, Segmented terahertz electron accelerator and manipulator (STEAM). Nat. Photonics 12, 336-342 (2018); doi:10.1038/s41566-018 -0138-z (cit. on p. 122).

406. D. Ehberger, K. J. Mohler, T. Vasileiadis, R. Ernstorfer, L. Waldecker, P. Baum, Terahertz Compression of Electron Pulses at a Planar Mirror Membrane. Phys. Rev. Appl. 11, 024034 (2019); doi:10.1103/PhysRevApplied.11.024034 (cit. on p. 122). 


\section{Author contributions, publications, conferences and awards}

The present cumulative thesis comprises the following articles, reprinted in Chapters 4 and 5, that fulfill the requirements for a cumulative thesis according to the doctoral degree regulations of the PhD program ProPhys at the Georg-August University School of Science (GAUSS):

- Th. Danz, Q. Liu, X. D. Zhu, L. H. Wang, S. W. Cheong, I. Radu, C. Ropers, R. I. Tobey, Structural and magnetic characterization of large area, free-standing thin films of magnetic ion intercalated dichalcogenides $\mathrm{Mn}_{0.25} \mathrm{TaS}_{2}$ and $\mathrm{Fe}_{0.25} \mathrm{TaS}_{2}$. J. Phys. Condens. Matter 28, 356002 (2016); doi:10.1088/0953-8984/28/35/356002 [see Ref. (67)].

- Th. Danz, T. Domröse, C. Ropers, Ultrafast nanoimaging of the order parameter in a structural phase transition. Science 371, 371-374 (2021); doi:10.1126/science.abd2774 [see Ref. (68)].

The publications listed above present original work by the author. The licenses of both journals, Journal of Physics: Condensed Matter and Science, prohibit reprinting the version of record in electronic theses. In this electronic version of the thesis published in the eDiss repository of the Göttingen State and University Library (SUB), the accepted manuscripts are printed instead. No substantial changes were made to the text or figures in the copyediting process.

The experimental work described in the first publication was partially conducted during a research stay in the Optical Condensed Matter Physics group of Assistant Professor R. I. Tobey at the University of Groningen. X. D. Zhu provided the $\mathrm{Mn}_{0.25} \mathrm{TaS}_{2}$ crystals while $\mathrm{Fe}_{0.25} \mathrm{TaS}_{2}$ was supplied by L. H. Wang and S. W. Cheong. Th. Danz prepared thin films of both materials using ultramicrotomy. MPMS measurements were conducted by Q. Liu and Th. Danz. Synchrotron work was done in collaboration with Q. Liu and R. I. Tobey, and supported by I. Radu. Th. Danz evaluated the synchrotron data in collaboration with Q. Liu, R. I. Tobey and C. Ropers. Th. Danz and R. I. Tobey wrote the manuscript. All authors discussed the results and their interpretation. Further non-author contributions are listed in the "Acknowledgments" section of Chapter 4. 
The research reported in the second publication has partially been done in collaboration with T. Domröse as part of his master's thesis (378). All contributions are listed in detail in the “Acknowledgments" section of Chapter 5.

\section{Publications}

The following articles originated over the course of this work and are not a direct part of the cumulative thesis:

- A. Feist, N. Bach, N. Rubiano da Silva, Th. Danz, M. Möller, K. E. Priebe, T. Domröse, J. G. Gatzmann, S. Rost, J. Schauss, S. Strauch, R. Bormann, M. Sivis, S. Schäfer, C. Ropers, Ultrafast transmission electron microscopy using a laser-driven field emitter: Femtosecond resolution with a high coherence electron beam. Ultramicroscopy 176, 63-73 (2017); doi:10.1016/j.ultramic.2016.12.005 [see Ref. (66)].

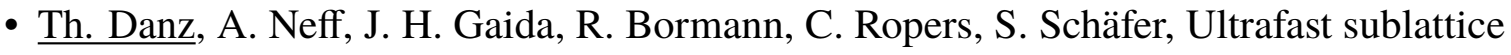
pseudospin relaxation in graphene probed by polarization-resolved photoluminescence. Phys. Rev. B 95, 241412 (2017); doi:10.1103/PhysRevB.95.241412 [see Ref. (197)].

- B. Buades, A. Picón, E. Berger, I. León, N. Di Palo, S. L. Cousin, C. Cocchi, E. Pellegrin, J. Herrero Martin, S. Mañas-Valero, E. Coronado, Th. Danz, C. Draxl, M. Uemoto, K. Yabana, M. Schultze, S. Wall, M. Zürch, J. Biegert, Attosecond state-resolved carrier motion in quantum materials probed by soft x-ray XANES. Appl. Phys. Rev. 8, 011408 (2021); doi:10.1063/5.0020649 [see Ref. (345)].

\section{Conference talks}

- Ultrafast transmission electron microscopy using laser-triggered field emitters, DPG Spring Meeting (SKM), Dresden, Germany (2017).

- Real-space order parameter mapping using ultrafast transmission electron microscopy, DPG Spring Meeting (SKM), Regensburg, Germany (2019).

- Ultrafast nano-imaging of the order parameter in a structural phase transition, Femtosecond Electron Imaging and Spectroscopy 4 (FEIS 4), Lincoln, NE, USA (2019).

- Ultrafast Transmission Electron Microscopy Using Laser-Triggered Field Emitters, Germany-Japan Joint Seminar on Advanced Electron Microscopy, Nagoya, Japan (2019). Invited talk. 
- Ultrafast nano-imaging of the order parameter in a structural phase transition, 5th International Conference on Ultrafast Structural Dynamics (ICUSD), Daejeon, South Korea (2019).

- Ultrafast nano-imaging of the order parameter in a structural phase transition, EMAG 2020: Microscopy Enabled by Direct Electron Detection, virtual conference (2020).

- Ultrafast nano-imaging of the order parameter in a structural phase transition, Virtual Early Career European Microscopy Congress (EMC), virtual conference (2020).

- Ultrafast nanoimaging of the order parameter in a structural phase transition, Microscopy and Microanalysis (M\&M), virtual conference (2021).

- Ultrafast nanoimaging of the order parameter in a structural phase transition, Microscopy Conference (MC), virtual conference (2021).

\section{Conference posters}

- Free-Standing Thin Films of Magnetic Intercalated Dichalcogenides studied by XMCD and ultrafast TEM, DPG Spring Meeting (SKM), Regensburg, Germany (2016).

- Large Area, Free-Standing Thin Films of Magnetic Ion Intercalated Dichalcogenides $\mathrm{Mn}_{0.25} \mathrm{TaS}_{2}$ and $\mathrm{Fe}_{0.25} \mathrm{TaS}_{2}$ for Ultrafast Transmission Electron Microscopy Experiments, Imaging with Femtosecond Electrons and X-ray pulses (IFEXS), Trieste, Italy (2016).

- Polarized hot carrier photoluminescence in graphene, DPG Spring Meeting (SKM), Dresden, Germany (2017).

- Ultrafast transmission electron microscopy applied to phase transitions in correlated materials, Microscopy Conference (MC), Lausanne, Switzerland (2017).

- Ultrafast transmission electron microscopy using laser-triggered field emitters, DPG Spring Meeting (SKM), Berlin, Germany (2018).

- Phase transitions in correlated materials probed by ultrafast transmission electron microscopy, Gordon Research Conference and Seminar (GRC/GRS): Ultrafast Phenomena in Cooperative Systems, Galveston, TX, USA (2018).

- Ultrafast nano-imaging of the order parameter in a structural phase transition, International Workshop on Correlated Dynamics in Energy Conversion (IWCE), Göttingen, Germany (2019). 
- Ultrafast nano-imaging of the order parameter in a structural phase transition, Gordon Research Conference and Seminar (GRC/GRS): Ultrafast Phenomena in Cooperative Systems, Barga, Italy (2020).

\section{Awards and scholarships}

- Doctoral scholarship by the German Academic Scholarship Foundation (2016-2019).

- "Best Student Presentation" award at the EMAG 2020: Microscopy Enabled by Direct Electron Detection conference hosted by the Electron Microscopy and Analysis Group (EMAG) of the Institute of Physics (IOP) (2020).

- "Student Award" at the Microscopy and Microanalysis (M\&M) conference hosted by the Microscopy Society of America (MSA) and the Microanalysis Society (MAS) (2021). 


\section{Danksagung}

In dieser Stelle möchte ich mich bei all denjenigen bedanken, die einen Beitrag zum Entstehen dieser Dissertation geleistet haben. Insbesondere gilt dieser Dank Prof. Dr. Claus Ropers, der mir die Möglichkeit der Promotion eröffnet hat und durch exzellente Betreuung und inspirierenden Austausch maßgeblich zum Erfolg der Arbeit beigetragen hat. Die enormen Freiheiten und das Vertrauen, das wir als Doktoranden in unserer Arbeitsgruppe genießen, haben persönlich und fachlich auf außerordentliche Weise zu meiner Weiterentwicklung beigetragen. Zugleich waren die zahlreichen Besuche nationaler und internationaler Konferenzen auf wissenschaftlicher wie touristischer Ebene eine große Bereicherung.

Weiterhin danke ich meinem Zweitbetreuer Prof. Dr. Christian Jooß sowohl für die interessierten Gespräche als auch für die Übernahme des Korreferats. Ich freue mich sehr über diese Konstante in meiner wissenschaftlichen Laufbahn seit Anfertigung meiner Bachelorarbeit am Institut für Materialphysik.

Dem gesamten UTEM-Team möchte ich für die tolle, kompetente und engagierte Zusammenarbeit danken. Über die Jahre hat jeder UTEM-Kollege auf die eine oder andere Weise zum Gelingen meines Projekts beigetragen und einige Kollegen sind in dieser Zeit zu Freunden geworden. Dies gilt insbesondere für Armin Feist, Marcel Möller, John Gaida, Till Domröse, Karin Ahlborn und Nora Bach, die mir neben aller gemeinsamer Alberei jederzeit mit Rat und Tat im und außerhalb des Labors zur Seite standen. Besonders bei Till möchte ich mich für die produktive Zusammenarbeit am Dunkelfeld-Projekt sowohl während seiner Masterarbeit als auch zu Beginn seiner Promotion bedanken. Armin, John, Till und Nora haben die Doktorarbeit Korrektur gelesen und allerlei konstruktive Hinweise gegeben - vielen Dank!

Insgesamt habe ich die offene und freundliche Atmosphäre in der Arbeitsgruppe und dem gesamten IV. Physikalischen Institut seit Beginn meiner Masterarbeit überaus zu schätzen gelernt. Mit etwas Wehmut blicke ich auf die vielen gemeinsamen Ausflüge und Feiern in den vergangenen Jahren zurück und hoffe sehr, dass auch wieder ,normalere Zeiten“ anbrechen. 
Außerdem danke ich meinem abenteuerlustigen Bürokollegen Oskar ganz herzlich für Mittagsspaziergänge bei Wind und Wetter, anregende Nasenstupser und motivierende Schnarchkonzerte direkt unter meinem Schreibtisch \%. Meinen Eltern danke ich für das beständige Interesse an meiner Arbeit und die Unterstützung während meines Studiums einschließlich der Promotion. Nicht zuletzt gilt mein ganz besonderer Dank meiner Frau Patrizia, die stets bedingungslos an meiner Seite steht, auch wenn sie im Laufe der Promotion einen erheblichen Anteil der Last unserer Fernbeziehung mitzutragen hatte. Ich freue mich auf die Zukunft mit Dir! 